\title{
Vertical Fluxes in the Upper Ocean
}

\author{
by \\ Mara Freilich \\ ScB, Brown University (2015)
}

Submitted to the Department of Earth, Atmospheric and Planetary Sciences in partial fulfillment of the requirements for the degree of

Doctor of Philosophy

at the

MASSACHUSETTS INSTITUTE OF TECHNOLOGY

and the

\section{WOODS HOLE OCEANOGRAPHIC INSTITUTION}

September 2021

(C)2021 Mara A. Freilich.

All rights reserved.

The author hereby grants to MIT and WHOI permission to reproduce and to distribute publicly paper and electronic copies of this thesis document in whole or in part in any medium now known or hereafter created.

Author

Department of Earth, Atmospheric and Planetary Sciences Massachusetts Institute of Technology \& Woods Hole Oceanographic Institution

June 9, 2021

Certified by

Amala Mahadevan

Senior Scientist Woods Hole Oceanographic Institution Thesis Supervisor

Accepted by

Glenn Flierl

Chairman, Joint Committee for Physical Oceanography Massachusetts Institute of Technology Woods Hole Oceanographic Institution 


\title{
Vertical Fluxes in the Upper Ocean
}

by

\author{
Mara Freilich
}

\author{
Submitted to the Department of Earth, Atmospheric and Planetary Sciences \\ Massachusetts Institute of Technology \\ \& Woods Hole Oceanographic Institution \\ on June 9, 2021, in partial fulfillment of the \\ requirements for the degree of \\ Doctor of Philosophy
}

\begin{abstract}
Oceanic fronts at the mesoscale and submesoscale are associated with enhanced vertical motion, which strengthens their role in global biogeochemical cycling as hotspots of primary production and subduction of carbon from the surface to the interior. Using process study models, theory, and field observations of biogeochemical tracers, this thesis improves understanding of submesoscale vertical tracer fluxes and their influence on carbon cycling. Unlike buoyancy, vertical transport of biogeochemical tracers can occur both due to the movement of isopycnals and due to motion along sloping isopycnals. We decompose the vertical velocity below the mixed layer into two components in a Lagrangian frame: vertical velocity along sloping isopycnal surfaces and the adiabatic vertical velocity of isopycnal surfaces and demonstrate that vertical motion along isopycnal surfaces is particularly important at submesoscales $(1-10 \mathrm{~km})$. The vertical flux of nutrient, and consequently the new production of phytoplankton depends not just on the vertical velocity but on the relative time scales of vertical transport and nutrient uptake. Vertical nutrient flux is maximum when the biological timescale of phytoplankton growth matches the vertical velocity frequency. Export of organic matter from the surface and the interior requires water parcels to cross the mixed layer base. Using Lagrangian analysis, we study the dynamics of this process and demonstrate that geostrophic and ageostrophic frontogenesis drive subduction along density surfaces across the mixed layer base. Along-front variability is an important factor in subduction. Both the physical and biological modeling studies described above are used to interpret observations from three research cruises in the Western Mediterranean. We sample intrusions of high chlorophyll and particulate organic carbon below the euphotic zone that are advected downward by 100 meters on timescales of days to weeks. We characterize the community composition in these subsurface intrusions at a lateral resolution of $1-10 \mathrm{~km}$. We observe systematic changes in community composition due to the changing light environment and differential decay of the phytoplankton communities in low-light environments, along with mixing. We conclude that advective fluxes could make a contribution to carbon export in subtropical gyres that is equal to the sinking flux.
\end{abstract}

Thesis Supervisor: Amala Mahadevan

Title: Senior Scientist

Woods Hole Oceanographic Institution 


\section{Acknowledgments}

The land on which this thesis was drafted is the traditional unceded territory of the Wampanoag Nation. We acknowledge the painful history of genocide and forced occupation of their territory, and we honor and respect the many diverse indigenous people connected to this land on which we gather from time immemorial.

Although only my name is listed as the author of this thesis, many people contributed to the production of this research and other work that formed part of my doctoral education but which is not directly in the following pages. There are many people to thank and naming them runs the risk of missing someone who I should thank. Keeping that risk in mind, I will attempt to acknowledge the relationships that forms the foundation of this work, just as I have, almost certainly imperfectly, cited the intellectual foundations of my work in the rest of this thesis. If you are reading this, you most likely contributed in some way even if you are not named here. I hope to have the opportunity to thank you and acknowledge your contributions.

Amala Mahadevan made this research possible through exceptional support and guidance. Thank you for your brilliant collaboration throughout and before my PhD and compassionate support of me as a scientist. Thank you to my thesis committee, Glenn Flierl, Alexandra Worden, and Eric D'Asaro for your enthusiasm and discerning insights about the essence of scientific contributions. I would also like to thank my other collaborators and scientific communities including the Mahadevan lab past and present - Jing He, Mathieu Dever, Sebastian Essink, Gualtiero Spiro Jaeger, Kate Lowry, Cynthia Wu, Roger Wu, and Katy Abbott - Eva Alou, Andrea Cabornero, John Allen, Simón Ruiz, Shaun Johnston, Tom Farrar, Ananda Pascual, Eugenio Cutolo, HM Aravind, and the whole CALYPSO science team, Ben van Mooy, Worden lab members - Camille Poirier, Lisa Sudek, Bente Gardeler, Chang Jae Choi. I want to express particular gratitude to Pierre Chabert, Kausalya Mahadevan, Alex Beyer, Margaret Conley, HM Aravind, and Salvador Vieira for their assistance with sampling through long nights and days at sea at times in rough conditions.

I would like to thank my collaborators on research I carried out during my $\mathrm{PhD}$ that is not in this dissertation but which contributed to my growth as a scientist and scholar; Raffaele Ferrari, Alexandre Mignot, Abigail Plummer, Federico Toschi, Roberto Benzi, Yoana Guzman Hernandez, Rohini Shivamoggi, Meghana Raganathan, Margaret Duffy, Julia Wilcots, Lyssa Freese, Astrid Pacini, Christina Hernandez, Kevin Archibald, Nicolas Muñoz, Juan Faundez. Pablo Marquet, Rolando Robelledo, Sergio Navarrete, Evie Weiters, Maria Jose Orellana, and Clara Arboleda who welcomed me in Chile as a Fulbright fellow. The Climate Changed team - Jessica Varner, Irmak Turan, Lizzie Yarina, and Miho Mazereeuw whose collaboration has stretched the ways that I think about science and my own ocean modeling work. Mike Neubert, Holly Moeller and everyone else who participated in ACKME.

Oceanography has a strong and growing infrastructure to collect data that is shared freely. This dissertation has benefited immensely from that data collection and careful curation including by NASA, ONR, Argo, the Bermuda Atlantic Time Series, the Hawaii Ocean Timeseries, and the Ocean Observatories Initiative.

A $\mathrm{PhD}$ involves becoming a part of scientific communities. WHOI academic programs office and EAPS headquarters including Meg Tivey, Jim Yoder, Kris Kipp, Lea Fraser, Christine Charette, Delia Oppo, Julia Westwater, Ed Boyle, Brandon Milardo, Megan Jordan, and Roberta Allard provided fundamental support that made this dissertation possible. I have had the privilege of navigating and interrogating scientific communities alongside other 
early career scholars including my PhD cohorts in PAOC and the Joint Program as well as the Society for Women in Marine Science steering committee.

This work would not be possible without the immense support from my family, including my parents Steven Freilich and Kathryn Pearlstine who inspire me as a scientist and nutured my interests in oceanography, my partner, Trevor Culhane, and friends near and far, especially those who welcomed me to Boston and helped me make a home here. Thank you for everything.

The work in this dissertation was funded by a NDSEG fellowship, Martin Fellowship, Grassle fellowship, Montrym grant, WHOI Academic Programs Office, and Office of Naval Research CALYPSO DRI grant N00014-16-1-3130. 


\section{Contents}

1 Introduction $\quad 25$

2 Decomposition of vertical velocity for nutrient transport in the upper $\begin{array}{ll}\text { ocean } & \mathbf{3 7}\end{array}$

2.1 Introduction . . . . . . . . . . . . . . . . . . . . . 38

2.2 Vertical velocity components . . . . . . . . . . . . . . . . . 42

2.2.1 Decomposition . . . . . . . . . . . . . . . . . . . 42

2.2 .2 Scaling relationships . . . . . . . . . . . . . . . . 44

2.3 Methods . . . . . . . . . . . . . . . . . . . . 46

2.3.1 Model setup . . . . . . . . . . . . . . . . . . . . . 47

2.3.2 Decomposition of vertical velocity . . . . . . . . . . . . . . . . . . . . . . . . . . . . . . . 48

2.3.3 Biological model . . . . . . . . . . . . . . . . . . . . . . . . . . . . . 49

2.4 Results . . . . . . . . . . . . . . . . . . . 50

2.4.1 Effects of varying isopycnal slope . . . . . . . . . . . . . . 50

2.4 .2 Along-isopycnal vertical velocity . . . . . . . . . . . . . 52

2.4 .3 Nutrient fluxes . . . . . . . . . . . . . . . . . . . . . 57

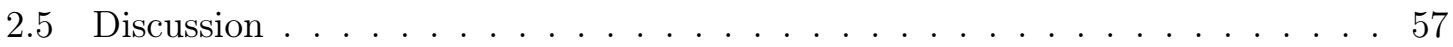

2.5.1 Global context . . . . . . . . . . . . . . 57

2.5.2 Along-isopycnal transport . . . . . . . . . . . . . . . . . . . . 59

2.5.3 Spatial scales . . . . . . . . . . . . . . . . . 60

2.5.4 Nutrient fluxes . . . . . . . . . . . . . . . . . . . 61

2.6 Conclusions . . . . . . . . . . . . . . . . . . . . 62

3 Biophysical feedbacks of phytoplankton growth on nutrient supply and $\begin{array}{ll}\text { diversity } & 65\end{array}$

3.1 Introduction . . . . . . . . . . . . . . . . . 65

3.2 Nutrient supply and uptake . . . . . . . . . . . . . . . . . 67

3.2.1 Dependence of flux on the frequency of vertical velocity and uptake rate 68

3.2.2 Nutrient fluxes in an oligotrophic eddy field . . . . . . . . . . . . . . 69

3.3 Lagrangian description of nutrient flux . . . . . . . . . . . . . . . . 72

3.3.1 Theoretical dependence of flux on biological rate . . . . . . . . . . . 72

3.3.2 Vertical velocity characteristics and biophysical fluxes . . . . . . . . 72

3.4 Discussion . . . . . . . . . . . . . . . . . . 76

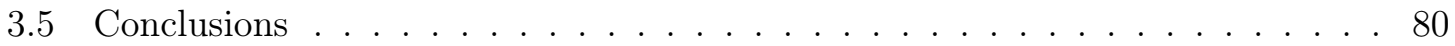

3.6 Methods . . . . . . . . . . . . . . . . . . . . . 80

3.6.1 Physical model . . . . . . . . . . . . . . . . . . 80

3.6 .2 Biological model . . . . . . . . . . . . . . . . . 80 
4 Coherent pathways for subduction from the surface mixed layer at ocean $\begin{array}{lr}\text { fronts } & \mathbf{8 3}\end{array}$

4.1 Introduction . . . . . . . . . . . . . . . . . . 84

4.2 Observational motivation . . . . . . . . . . . . . . 87

4.3 Theoretical background . . . . . . . . . . . . . . . . . 90

4.4 Methods . . . . . . . . . . . . . . . . . . . . . . 92

4.4 .1 Model set up . . . . . . . . . . . . . . . . . 93

4.4 .2 Particle tracking . . . . . . . . . . . . . . . . . . . . . . . 94

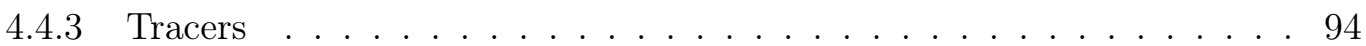

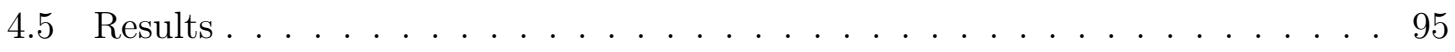

4.5.1 Subduction rate . . . . . . . . . . . . . . . . . . . . . 95

4.5.2 Coherence of subduction . . . . . . . . . . . . . . . . . 96

4.5.3 Lagrangian description of subduction dynamics . . . . . . . . . . . . 98

4.5.4 Subduction by submesoscale features . . . . . . . . . . . . . . . 102

4.6 Discussion . . . . . . . . . . . . . . . . . . . . . . . . . . 108

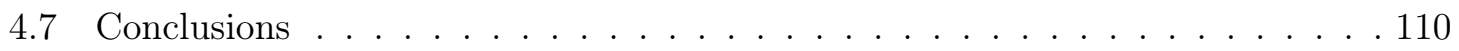

5 Advective carbon export in an oligotrophic gyre by coupled mesoscalesubmesoscale dynamics 111

5.1 Introduction . . . . . . . . . . . . . . . . . . . . 111

5.2 Hydrographic and biogeochemical setting . . . . . . . . . . . . 113

5.3 Observations of subduction . . . . . . . . . . . . . . . . 118

5.4 Biophysical determinants of carbon flux . . . . . . . . . . . . . 123

5.4 .1 Magnitude of carbon subduction . . . . . . . . . . . . . . . 123

5.4 .2 Contribution to export by small cells . . . . . . . . . . . . . . . 125

5.4 .3 Community composition . . . . . . . . . . . . . . . 126

5.4 .4 Ecological effects of subduction . . . . . . . . . . . . . . . 133

5.5 How much does frontal subduction contribute to POC export in oligotrophic gyres? . . . . . . . . . . . . . . . . . . . . . . . . . . 139

5.6 Conclusion . . . . . . . . . . . . . . . . . . . . . . . . . . . 140

5.7 Methods . . . . . . . . . . . . . . . . . . 140

5.7 .1 Sampling strategy . . . . . . . . . . . . . . . . . 140

5.7 .2 Temperature and salinity . . . . . . . . . . . . . . . . . 142

5.7 .3 Nutrients . . . . . . . . . . . . . . . . . . . . . . . . . . . . 142

5.7 .4 Chlorophyll . . . . . . . . . . . . . . . . . . . . . . . . 142

5.7 .5 Oxygen . . . . . . . . . . . . . . . . . . . . . . 142

5.7 .6 Photosystem II efficiency $\left(\mathrm{F}_{\mathrm{v}} / \mathrm{F}_{\mathrm{m}}\right) \ldots \ldots . \ldots . \ldots . \ldots . \ldots 143$

5.7 .7 Respiration rate . . . . . . . . . . . . . . . . . . . . 143

5.7 .8 Biological community composition . . . . . . . . . . . . . . 143

5.7 .9 Statistical analyses . . . . . . . . . . . . . . . . . 145

5.7 .10 Computing particulate organic carbon (POC) . . . . . . . . . 145

5.7 .11 Computing POC flux . . . . . . . . . . . . . . 147

$\begin{array}{llr}6 & \text { Conclusions } & 149\end{array}$ 
A Derivations of flux dependence on biological timescale and model assumptions

A.1 Biological model and discussion of assumptions . . . . . . . . . . . . . 159

A.2 General theory . . . . . . . . . . . . . . . . . . . 161

A.3 Simplified physics . . . . . . . . . . . . . . . . . 161

A.3.1 Flux . . . . . . . . . . . . . . . . . . . . . . . . 162

A.3.2 Correlation between vertical velocity and nutrient anomaly . . . . . 163

A.4 Fit between model and theory . . . . . . . . . . . . . . . . 163

A.5 General analysis of the maximum flux . . . . . . . . . . . . 166

B Patchiness as a function of biological rate 169

C Observations of flux dependence on biological timescale 173

C.1 Model set-up . . . . . . . . . . . . . . . . . . . . . . . 173

C.2 North Pacific comparison . . . . . . . . . . . . . . . . . . 173

$\begin{array}{ll}\text { D Tracer analysis of mixed layer subduction } & 179\end{array}$

E Estimation of POC from flow cytometry and bio-optical properties $\quad 181$

$\begin{array}{lll}\text { F Supplemental biological data analysis figures } & 183\end{array}$ 


\section{List of Figures}

1-1 Schematic illustration of a front. A front is the confluence between two water masses that have distinct properties, here indicated by the distinct colors. As the front meanders and develops other instabilities, upwelling and downwelling is generated at the front (arrows). This upwelling and downwelling transports heat and biogeochemical tracers. This dissertation uses data collected from ship-board surveys across fronts. One of the tools is a CTD rosette, which is shown in the water below the ships. The rosette has Niskin bottles to collect seawater from below the surface. The ships and the front are not scaled proportionally. Figure created by Eric Taylor, WHOI graphics for Freilich (2018). . . . . . . . . . . . . . . . . . 26

1-2 MODIS satellite observation of chlorophyll on March 11, 2020 in the Western Mediterranean Sea. The chlorophyll distributions show influence of upwelling and stirring. . . . . . . . . . . . . . . . . . . . . 3

1-3 Nutrient as a tracer of fluid motions on a constant density (isopycnal) surface. The nutrient concentration is affected by both biological reactions and physical transport. The surface has variations in height such that motion on this surface could have a vertical component. This figure was generated from the model analyzed in chapters 2 and $3 \ldots \ldots \ldots 33$

2-1 Calculation of the vertical component of the along-isopycnal velocity, $w_{i s o} . w$ is the total vertical velocity and $u_{d i v}$ is the divergent part of the horizontal velocity. $\Gamma=\tan ^{-1}(|\nabla h|)$. The light blue lines show the projection of $u_{\text {div }}$ and $w$ onto the isopycnal surface and sum to $u^{\sigma}$ in equation 2.1. The dashed green line is the vertical component of the along-isopycnal velocity. . . . . . . 43

2-2 Model initialization. (a) The model is forced with a positive heat flux in the south and a negative heat flux in the north. (b) Three-dimensional view of the model domain. The model is initialized with three density profiles in order to create two fronts. (c) Initial domain-scale isopycnal slopes. . . . . . . 48

2-3 Surface relative vorticity (normalized by $f$ ) in color, overlaid with density contours (black), from the $1 \mathrm{~km}$ (Top row) and $4 \mathrm{~km}$ (Lower row) resolution models on day 10 of the analysis period. The columns, from left to right, show fields from the models with weak, medium, and steeply sloping isopycnals, generated with horizontal temperature variations of $2^{\circ} \mathrm{C}, 3^{\circ} \mathrm{C}$, and $4^{\circ} \mathrm{C}$ over the domain. Regions within $32 \mathrm{~km}$ of the north and south boundaries are eliminated from the analysis and are not shown. . . . . . . . . . . . 51 
2-4 (a) Root mean squared isopycnal slopes calculated by averaging the density into $50 \mathrm{~km} \times 50 \mathrm{~km}$ bins in the horizontal during the analysis period. The upper 100 meters are not shown because the isopycnal slope is undefined in the mixed layer. (b) Mean nutrient concentration between 0 and $500 \mathrm{~m}$ during the analysis period. . . . . . . . . . . . . . . . . 52

2-5 Vertical velocity and nutrient concentration at 115 days on the $\sigma=25.9$ isopycnal surface in the $1 \mathrm{~km}$ resolution model initialized with $\Delta T=2^{\circ} \mathrm{C}$. The isopycnal surface spans the depth range -150 to -50 meters; (a) $w_{\text {iso }}$ and (b) $w_{\text {uplift }}$ in $\mathrm{mm}-\mathrm{s}^{-1}$, and (c) nutrient concentration in $\mu \mathrm{mol} \mathrm{kg}{ }^{-1}$. The euphotic depth $(1 \%$ light level $)$ is $125 \mathrm{~m} \ldots \ldots . \ldots . . \ldots 53$

2-6 (a) Ratio of root median squared $w_{\text {iso }}$ to root median squared $w$ below the mixed layer and above 135 meters, plotted against root mean squared $\frac{M^{2}}{N f}$, evaluated between the base of the mixed layer and a depth of $135 \mathrm{~m}$ where, the aspect ratio $\frac{H}{L}=\frac{f}{N} \ll 1$. All of the averages are taken over the model domain and over the analysis period of 30 days. The slope of the best fit line is 2.58 and the intercept is -0.13 with an $R^{2}=0.98$. The diamonds are the $4 \mathrm{~km}$ resolution simulations while the circles are the $1 \mathrm{~km}$ resolution simulations. The open symbols are the ratio of the nutrient fluxes due to each of the vertical velocity components $\frac{\overline{w_{i s o \mathcal{N}^{\prime}}}}{w_{\mathcal{N}^{\prime}}} \ldots \ldots \ldots \ldots \ldots$. . . . . . . . . 5 5

2-7 Probability density functions (PDFs) on a log frequency axis: (a-c) Vertical velocity components near the euphotic depth (100-135 meters), and (d-f) vertical nutrient flux components at the same depth. (a) Vertical velocity $(w)$, (b) along-isopycnal vertical velocity $\left(w_{i s o}\right)$, (c) isopycnal uplift velocity $\left(w_{\text {uplift }}\right),(\mathrm{d})$ nutrient flux due to total vertical velocity $\left(w \mathcal{N}^{\prime}\right),(\mathrm{e})$ nutrient flux due to along-isopycnal vertical velocity $\left(w_{\text {iso }} \mathcal{N}^{\prime}\right)$, (f) nutrient flux due to isopycnal uplift vertical velocity $\left(w_{\text {uplift }} \mathcal{N}^{\prime}\right)$. In each case, PDFs can be compared for the $4 \mathrm{~km}$ and $1 \mathrm{~km}$ resolution models and the different isopycnal slopes $\left(\Delta \mathrm{T}=2^{\circ}, 3^{\circ}, 4^{\circ} \mathrm{C}\right) \ldots \ldots \ldots \ldots \ldots \ldots \ldots \ldots$

2-8 Spectrum of $w_{\text {uplift }}$ and $w_{\text {iso }}$ on the $\sigma=25.9$ isopycnal surface for each of the various isopycnal slopes $\left(M^{2} / N^{2}\right)$ modeled using a horizontal temperature difference $\Delta \mathrm{T}=2^{\circ}, 3^{\circ}$ and $4^{\circ} \mathrm{C}$. (a) $1 \mathrm{~km}$ resolution models (b) $4 \mathrm{~km}$ resolution models. . . . . . . . . . . . . . . . . . . . 56

2-9 Scaling factor $\frac{M^{2} L}{N^{2} H}$ is calculated at $150 \mathrm{~m}$ depth from global, $1 / 48^{\circ}$-resolution MITgcm model fields on (a, c) January 15, 2012 and (b, d) June 15, 2012. Areas where the mixed layer is deeper than 150 meters or where the total water column depth is less than 150 meters are shaded white. The inverse aspect ratio of the velocity is calculated as $\frac{L}{H}=\sqrt{\frac{\partial u^{2}}{\partial z}+\frac{\partial v^{2}}{\partial z}} / \sqrt{\frac{\partial u}{\partial y}+\frac{\partial v}{\partial x}^{2}}$. The computed ratio is smoothed using a Gaussian filter with a $120 \mathrm{~km}$ window in figures (a) and (b) and a $12 \mathrm{~km}$ window in figures (c) and (d). Simulations provided by Christopher N. Hill. . . . . . . . . . . . . . . . 58

3-1 Schematic showing the relationship between vertical velocity, nutrient anomaly and nutrient flux. . . . . . . . . . . . . . . . . . . . 69

3-2 Snapshots of vertical velocity, nutrient anomaly, and vertical nutrient fluxes on model day 135 (A) Vertical velocity at $z=-117 \mathrm{~m}$. (B and C) Lower cube is nutrient anomaly, the upper panel is the vertical nutrient flux at $z=-117 \mathrm{~m}$ with uptake rates (B) $\lambda=0.015$ day $^{-1}$ and (C) $\lambda=1.5$ day $^{-1} . \quad 70$ 
3-3 Frequency spectra averaged over all Lagrangian particles with a given uptake rate that maximizes the nutrient flux. . . . . . . . . . . . . . . . 73

3-4 (A) Distribution of rates $\lambda$ that maximizes the nutrient flux. (B) Nutrient flux as a function of uptake rate on Lagrangian trajectories (orange curve) and theoretical expectation of the nutrient flux from equation 3.3 (green curve). The solid line is the median of all trajectories; shading shows the interquartile range. The grey bar on the ordinate shows the interquartile range of the vertical eddy diffusion in the absence of biological uptake. The dots on the flux $=0$ axis indicate the uptake rates shown in Fig. 3-3. . . . . . . . . . . . 74

3-5 (A) Uptake rate that maximizes the nutrient flux for each trajectory plotted at the trajectory origin location. (B) Nutrient flux at the uptake rate that maximizes the flux. The black points are excluded from the analysis in figure 3-4 because they leave the nutricline. The contours are density with the dashed contours being lighter densities. . . . . . . . . . . . . . . 75

3-6 (upper) Histogram of carbon-specific growth rate estimated from primary production and cell counts at the Hawaii Ocean Time-series station ALOHA (lower) Temporal average of biological nutrient flux at $120 \mathrm{~m}$ as a function of

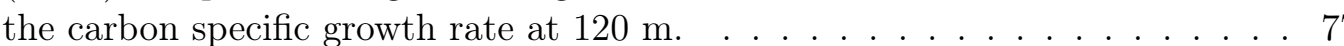

4-1 (a) MODIS Aqua satellite image of chlorophyll in the Alborán Sea on March 28,2019 . The geostrophic currents from AVISO (arrows) show the anticyclonic Western Alborán Gyre. The chlorophyll (in color) shows the influence of frontal dynamics at the gyre edge with frontal waves on the northern edge of the gyre. The CALYPSO cruise on the $N / O$ Pourquois Pas? surveyed the front transiting from south to north along the yellow line on March 30, 2019. (b) Transect of temperature (color) from an underway CTD with density contours at intervals of $0.1 \mathrm{~kg} / \mathrm{m}^{3}$. Subducted water masses are identified by temperature variations along an isopycnal surface. Two warm intrusions are highlighted by demarcating their bounding isopycnals in blue. (c) Transect of relative vorticity computed from the along-track gradient of the horizontal velocity normal to the ship section. Velocity measurements are from a vessel-mounted ADCP. . . . . . . . . . . . . . . . . . . . 88

4-2 Vertical velocity with density contours on day 47.5. The vertical velocity that is shown at the surface is the 5 meter vertical velocity. Vertical velocity is shown in meters/day, with a saturated color scale. . . . . . . . . . . . . 93

4-3 Positions $(x-y)$ of particles on the day that they subduct out of the mixed layer. The particles are colored by their relative vorticity, with red referring to cyclonic, and blue to anti-cyclonic vorticity. Density at $5 \mathrm{~m}$ depth is contoured (thin lines CI $=0.02 \mathrm{~kg} / \mathrm{m}^{3}$, thick lines CI $=0.2 \mathrm{~kg} / \mathrm{m}^{3}$ ). Dashed (solid) contours are lighter (denser) than the average surface density. Particles are subducting along the dense side of the fronts where the lateral buoyancy gradient is strongest, and most of the particles have cyclonic relative vorticity. The subduction locations are coherent and elongated in the along-front direction. . . . . . . . . . . . . . . . . 96 
4-4 (A) Vertical velocity and vertical transport isotropic wavenumber variance spectra. The black lines are the vertical velocity spectrum at (solid) 5 meters depth and (dashed) 40 meters depth. The colored lines show the spectra of the vertical transport at 40 meters depth calculated over a range of time intervals according to (4.5). The grey lines are guides for $k^{-1}$ and $k^{-3}$ slopes. (B) Vertical velocity at 40 meters depth. (C) Vertical transport over 10 days at 40 meters depth. . . . . . . . . . . . . . . . . . . . . . 97

4-5 Lagrangian evolution of dynamical quantities as water parcels are subducted. All trajectories are composited onto a shifted time axis where time zero is the subduction time of the water parcel (defined as the time when it moves from the mixed layer to 5 meters below the mixed layer depth). The solid line is the median value. The shaded region encloses the first and third quartiles of all subducted particles. Approximately $7 \%$ of all of the particles subduct. The dashed line shows the median of all particles that subduct faster than $20 \mathrm{~m} /$ day. The reference Coriolis frequency used to normalize properties is $f=10^{-4} \mathrm{~s}^{-1}$. (A) Stratification $\left(N^{2} / f^{2}\right)$ (left axis, green) and lateral buoyancy gradient $\left(M^{2} / f^{2}\right)$ (right axis, orange) (B) Relative vorticity normalized by the Coriolis frequency (left axis, green) and vertical velocity (right axis, orange) (C) Non-linear acceleration due to curved particle trajectories. The radius of curvature $(R)$ is calculated using velocities and accelerations averaged over 15 hours on particle trajectories. (D) Various contributions to frontogenesis, represented by the terms on the right hand side of Equation (4.2) integrated in time along particle trajectories. The lines show the median values of all subducted particles on a shifted time axis. Positive values are frontogenetic and negative values are frontolytic. The $\mathbf{Q}$-vector is split into geostrophic and ageostrophic components. The third term is frontogenesis by the vertical velocity ("vertical"). The fourth term is horizontal diffusion $\left(k_{h}\right)$. The fifth term is vertical diffusion $\left(k_{v}\right)$. (E) Two dimensional histogram showing the relationship between the vertical component of relative vorticity (normalized by $f$ ) and the vertical velocity on particles during the 20 hours prior to subduction. (F) Vertical and horizontal contributions to the PV. The purple line is the median of the total PV . . . . . . . . . . . . 100 
4-6 A submesocale filament as a pathway for subduction from the mixed layer. The evolution of a cyclonic filament is shown in panels A, B. (A) Surface relative vorticity normalized by the Coriolis frequency on days 47.5 (1) and 48.5 (2). The contour is $\sigma=27.35$. (B) PV on the $\sigma=27.35$ surface. The black contours are isopycnal height at 10 meter intervals. All particles shown have density within $0.01 \mathrm{~kg} / \mathrm{m}^{3}$ of the isopycnal surface and subduct below the mixed layer during their trajectory. The particles are colored by their relative vorticity. (C) Lower panel: Cross section of the vertical velocity in meters/day on day 47.5 along the black dashed line $\mathrm{T}^{\prime}-\mathrm{T}$ in panel $\mathrm{A}(1)$. Contours are isopycnals. The black contours $\sigma=27.8$. Upper panel: surface horizontal velocity in the along plane direction (x-axis) and cross plane direction. Downwards in the cross plane direction is out of the page (positive $\mathrm{x}$ direction). (D) Mean time-integrated frontogenetic forcing on the particles within the orange box in panels A,B. Positive slopes are frontogenetic and negative slopes are frontolytic. Contributions to frontogenesis are split into the terms on the right hand side of Equation (4.2). The black dot on the $\mathrm{x}$-axis indicates the time of subduction. . . . . . . . . . . . . . 103

4-7 Two specific pathways for subduction from the mixed layer form an intrapycnocline eddy (orange box) and cut-off cyclone (green box). (A) Surface relative vorticity normalized by the Coriolis frequency on days 49.5 (1) and 50.5 (2). The contour is $\sigma=27.8$. (B) PV on the $\sigma=27.8$ surface. The black contours are isopycnal height at 10 meter intervals. All particles shown have density within $0.01 \mathrm{~kg} / \mathrm{m}^{3}$ of the isopycnal surface and subduct below the mixed layer during their trajectory. The particles are colored with their relative vorticity. (C,D) Contributions to frontogenesis split into the terms on the right hand side of Equation (4.2). The forcing due to each term is integrated in time along each subducting particle trajectory. (C) Mean time-integrated frontogenetic forcing on the particles within the orange box in panels A,B (D) Mean time-integrated frontogenetic forcing on the particles within the green box in panels A,B. Positive slopes are frontogenetic and negative slopes are frontolytic. The black dot on the $\mathrm{x}$-axis indicates the time of subduction. (E, F) Cross sections on day 49.5 at the black dashed line on panels A,B. Contours are isopycnals. The black contour is $\sigma=27.8$. (E) Upper panel: surface horizontal velocity in the along plane direction (x-axis) and cross plane direction. Downwards in the cross plane direction is out of the page (positive $\mathrm{x}$ direction). Lower panel: Vertical velocity in meters per day. (F) PV with velocity vectors. The low PV anomaly between the orange markers is an intrapycnocline eddy formed by subduction. The green and orange triangles show the y locations of the green and orange boxes in panels A1,B1. . . . . . 104

5-1 Sampling locations. The contours show the sea surface height in May 2018. The arrows are provided to aid conceptual interpretation and show the direction of the currents. Points show the locations of CTD casts. Black lines connect CTD transects that took place within a short time period. . . . . . . 114 
5-2 Temperature-salinity diagrams with biogeochemical data. The grey points show temperature-salinity from the ship-board CTD from all cruises and are the same on every panel. Chlorophyll (top) and nitrate (bottom) measurements are shown from each research cruise (columns). Chlorophyll measurements are from the ship-board CTD fluorometer calibrated with in situ samples. Nitrate measurements are from the SUNA attached to the shipboard CTD, calibrated with in situ samples. Nitrate measurements from Calypso 2018 are only in situ samples. The temperature axis limits are different for each row. . . . . . . . . . . . . . . . . . . . . . 115

5-3 Distribution of intrusions in space during CLP19. The contours are sea surface height from AVISO. The Western Alborán Gyre is in the lower left and the anticyclonic eddy is in the upper right. (left) The grey shows the location of all underway CTD samples. In each cast where a intrusion was identified from the temperature-salinity properties, the depth of the top of the intrusion water in meters is plotted in color. (right) Sea surface density on the cruise track from the seawater intake thermosalinograph. . . . . . . . . . . . 116

5-4 Transect C2, as labeled on figure 5-1. The background shows the temperature from an underway CTD transect immediately prior to the CTD transect. The black contours are density. The chlorophyll concentration shown is from the CTD casts. The uCTD and CTD transects were a few hours apart so the features in temperature and chlorophyll are not perfectly aligned in depth due to internal waves and tides. . . . . . . . . . . . . . . . . . . . 117

5-5 Transects A, B2, and C3, as labeled on figure 5-1. Chlorophyll concentration from the EcoCTD. The density contours on sections B2 and C3 are $0.1 \mathrm{~kg} / \mathrm{m}^{3}$ apart. The thick density contours show the density of the intrusion observed in the transect. . . . . . . . . . . . . . . . . . . . . . 117

5-6 Average mixing length calculated on each cross-front transect using the spice variance on density surfaces. . . . . . . . . . . . . . . . . . . . . . 119

5-7 Model analysis of subduction. (A) Lagrangian particles (yellow) originated at $70 \mathrm{~m}$ and are subducted along an isopycnal surface. The white trajectories show the $x-y-z$ location history of the particles. The isopycnal surface is shown with temperature shading. Temperature is shown on the boundaries of the frame as well. (B-D) Histograms of the relationship between vertical and lateral displacement of water parcels. (B) particles initially at $70 \mathrm{~m}$ in a model with a stratified surface layer representing the June and July research cruises. The particles shown in panel (A) are a subset of the particles shown in panel B. (C) particles initially at $70 \mathrm{~m}$ in a model with $50 \mathrm{~m}$ deep mixed layer representing the March-April research cruise. (D) particles initially at $5 \mathrm{~m}$ in the model with a $50 \mathrm{~m}$ deep mixed layer. Statistics on this plot are from water parcels that leave the mixed layer. . . . . . . . . . . . . . 121 
5-8 POC export during CLP19. (A) Probability density functions of POC concentration within the intrusions (green) and POC concentration randomly sampled with the same depth distribution as the intrusions but outside the intrusions. The dashed line shows the geometric mean of the POC distribution in the gyre intrusion and background. The dotted line shows the mean of the POC distribution in the eddy intrusion and background. The grey shading shows the $95 \%$ confidence interval. These averages are also shown in panel (B). (B) Average POC profile in the gyre (dashed) and eddy (dotted). The green scatter points show the intrusion POC concentrations at each depth below $100 \mathrm{~m}$. The darker points are in the eddy and the lighter points are in the gyre. (C) Depth distribution of intrusion samples below $100 \mathrm{~m}$. . . . . . . 126

5-9 Samples from the intrusion in transect C2 (Figure 5-1). This transect cross section is shown in figure 5-4. In the cross section, the intrusion samples are shown in black dots. The black squares show the samples on the left and right sides of this figure, which are outside of intrusions and considered "background". The cell concentrations are derived from flow cytometry. The cell concentrations of the cyanobacteria ecotypes are estimated by mapping the relative abundance of ecotypes onto cell counts from flow cytometry. The yellow line shows the intrusion depth with the dot indicating the sample location and the bars showing the feature depth range. The blue line shows the average AOU within the intrusion. The numbers above the bars near 25, 30, and $35 \mathrm{~m}$ are $\mathrm{F}_{\mathrm{v}} / \mathrm{F}_{\mathrm{m}}$ measurements for those samples. . . . . . . . . . 127

5-10 POC estimated from flow cytometry, which captures the small photosynthetic and heterotrophic biomass plotted against the POC estimate from beam transmission, which captures a larger fraction of the total POC and non-biological particulate matter. The grey bars show the ranges of possible values based on the uncertainty in the conversion factor. The uncertainty is correlated between points. The first CLP18 transect was more coastal, ending near Moroccan territorial waters, which could explain the relatively low fraction of the POC that is in the small fraction. The black line is the 1:1 line.127

5-11 Profiles of the average POC concentration in each taxonomic group estimated from flow cytometry, separated by sampling year (lines). The scatter points show the POC concentration in the intrusions. . . . . . . . . . . . . . 128

5-12 Ordination of heterotrophic bacteria ASVs. (A) Non-metric multidimensional scaling (NMDS) of the Bray-Curtis distance between samples. The ordination used 3 NMDS axes (only two are shown) and resulted in a stress of 0.071. The sample feature is shown with the color of the point and the research cruise is shown in the shape of the point. (B) The first NMDS axis is plotted against the sample depth. (C) ASVs are aggregated at the genus level and all genera that comprise at least $2 \%$ of one sample are plotted. The color in the heatmap shows the $\mathrm{z}$-score of the relative abundance within the sample. Samples are arranged in the order of the first NMDS axis. . . . . . . . . . . 129 
5-13 Hierarchical clustering of samples from the intrusions, background below $90 \mathrm{~m}$, surface chlorophyll maximum, deep chlorophyll maximum, and lower deep chlorophyll maximum. Sample distance is Bray-Curtis distance and clusters are formed using average-linkage clustering. The first bar below the tree shows the feature that the sample came from, the second shows the sample depth, the third shows the sample nitrate concentration, and the fourth shows which research cruise. The heatmap shows the relative abundance of the 15 most common taxa in these samples. . . . . . . . . . . . . . . . . . . . . . 130

5-14 Observations of a intrusion at the Almeria-Oran front sampled during IRENE in July 2017. (A) POC concentration in each taxonomic group in intrusions (green) and background samples below $90 \mathrm{~m}$ (blue). (B) Difference between the geometric mean of intrusion and background POC concentrations in each taxonomic group and the total POC concentration estimated from flow cytometry. The t-test significance level of enhancement in the intrusion over the background is shown with the stars ${ }^{* * *}=\mathrm{p}<0.001,{ }^{* *}=\mathrm{p}<0.01,{ }^{*}=$

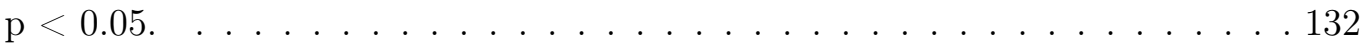

5-15 Observations of a intrusion at the Almeria-Oran front sampled during CLP18 in May-June 2018. (A) POC concentration in each taxonomic group in intrusions (green) and background samples below $100 \mathrm{~m}$ (blue). (B) Difference between the geometric mean of intrusion and background POC concentrations in each taxonomic group and the total POC concentration estimated from flow cytometry. The t-test significance level of enhancement in the intrusion over the background is shown with the stars ${ }^{* * *}=\mathrm{p}<0.001, * *=$ $\mathrm{p}<0.01,{ }^{*}=\mathrm{p}<0.05 . \ldots \ldots \ldots . \ldots \ldots \ldots$

5-16 Observations of intrusions at the Western Alboran Gyre sampled during CLP19 in March and April 2019. (A) POC concentration in each taxonomic group in intrusions (green) and background samples below $100 \mathrm{~m}$ (blue). (B) Difference between the geometric mean of intrusion and background POC concentrations in each taxonomic group and the total POC concentration estimated from flow cytometry. The t-test significance level of enhancement in the intrusion over the background is shown with the stars $* * *=p<0.001$, ${ }^{* *}=\mathrm{p}<0.01,{ }^{*}=\mathrm{p}<0.05 \ldots \ldots \ldots . \ldots \ldots \ldots$

5-17 Community similarity on a cross-frontal transect. The purple points show the Bray-Curtis distance from the sample at the intrusion tip at $23 \mathrm{~km}$ at the discrete sample locations. The background is AOU from the ecoCTD. . . . . 135

5-18 (A) Average fluorescence of Prochlorococcus cells at $692 \mathrm{~nm}$ quantified from flow cytometry. The green "subducted eddy" samples are from the intrusion on transect C3. The orange "downstream eddy" samples are from the chlorophyll maximum, intrusion, and background on transect $\mathrm{C} 4$. The purple "subducted gyre" are from intrusion samples on transects C1, C2, and C5. (B1) Average fluorescence of Prochlorococcus cells at $692 \mathrm{~nm}$ plotted in order of the average fluorescence of Prochlorococcus cells at $692 \mathrm{~nm}$ ("brightness rank"). In the other panel (B) samples, samples are plotted in the same order. (B2) Prochlorococcus cells per ml (B3) Relative proportion of Prochlorococcus $16 \mathrm{~S}$ amplicons classified in each ecotype. . . . . . . . . . . . . 136 
A-1 (top) Vertical nutrient flux as a function of growth rate for a range of vertical velocity frequencies. (bottom) Vertical nutrient flux as a function of vertical velocity frequency for a range of growth rates. The vertical flux is computed using the idealized model in equation A.16. . . . . . . . . . . . . . . . . . . 160

A-2 Histogram of the optimal nutrient flux calculated using equation A.21 ("theory") and on particles in the 3D model. The frequencies are normalized by the number of particles with a given optimum in the theoretical calculation. . 164

A-3 (A) Distribution of rates $\lambda$ that maximizes the nutrient flux. (B) Nutrient flux as a function of biological rate on Lagrangian trajectories (orange curve) and theoretical expectation of the nutrient flux from equation 3 (green curve). The solid line is the median of all trajectories shading shows the interquartile range. The grey bar on the ordinate shows the interquartile range of the vertical eddy diffusion in the absence of biological uptake. . . . . . . . . . . 165

A-4 Nutrient anomaly as a function of the depth anomaly (vertical distance from $\mathrm{z}=-155 \mathrm{~m}$ ) for a range of biological rates. . . . . . . . . . . . 165

A-5 (A) Distribution of rates $\lambda$ that maximizes the nutrient flux. (B) Nutrient flux as a function of biological rate on Lagrangian trajectories (orange curve) and theoretical expectation of the nutrient flux from equation 3 (green curve). The solid line is the median of all trajectories shading shows the interquartile range. The grey bar on the ordinate shows the interquartile range of the vertical eddy diffusion in the absence of biological uptake. . . . . . . . . . . . 167

A-6 Vertical velocity autocorrelation as a function of time. The grey curve shows the median and interquartile range of all trajectories. The colored lines show the median autocorrelation across all trajectories with a given biological rate the maximizes the flux. . . . . . . . . . . . . . . . . . . . . 168

A-7 First zero crossing of the vertical velocity autocorrelation. The biological rate on the abscissa is the rate the maximizes the vertical flux. The box plots show the distributions of first zero crossings across all trajectories. . . . . . . . . 168

B-1 (A) Wavenumber-frequency spectrum of the vertical kinetic energy at 117 meters. (B, C) Wavenumber-frequency of the biological reaction term $\left(\lambda c^{\prime}\right)$ at 117 meters with $(\mathrm{B})$ a relatively fast growth rate $(\lambda=0.75)$ and $(\mathrm{C})$ a relatively slow growth rate $(\lambda=0.075)$. ( $\mathrm{D}, \mathrm{E})$ Wavenumber-frequency of the new production, the positive part of $\lambda c^{\prime}$, at 117 meters with (D) a relatively fast growth rate $(\lambda=0.75)$ and $(\mathrm{E})$ a relatively slow growth rate $(\lambda=0.075)$. $(\mathrm{F}, \mathrm{G})$ Wavenumber-frequency of the nutrient flux $\left(\lambda c^{\prime}\right)$ at 117 meters with (F) a relatively fast growth rate $(\lambda=0.75)$ and $(\mathrm{G})$ a relatively slow growth rate $(\lambda=0.075)$. On each panel the grey horizontal line is at the inertial frequency. The grey diagonal line is a non-dispersive line with a phase speed of $17 \mathrm{~km} /$ day. The black contours are level curves of the vertical velocity wavenumber-frequency spectrum. . . . . . . . . . . . . 171

C-1 Chlorophyll as a function of depth observed at BATS in June (green) and $\frac{\mathrm{d} N_{0}}{\mathrm{~d} z} 175$ 
C-2 Model and observational nutrient profiles. The model equilibrium profile is the black line and the dashed line is a shifted profile to match the nutricline depth at HOT. The observational profiles are monthly mean profiles from the Bermuda Atlantic Time Series (blues) and Hawaii Ocean Timeseries (oranges). The colors vary from dark to light with month from January to December. The model profile has no gradient below the maximum euphotic depth so that there is no productivity below this depth, due to the model simplifications. The model has a larger nutrient gradient in the nutricline than do the observations at BATS. . . . . . . . . . . . . . . . . 175

C-3 From top to bottom, left to right, measured and computed quantities as a function of month, euphotic zone average carbon specific growth rate, carbon specific growth rate at 120 meters, euphotic zone average primary production, average nutrient anomaly in the euphotic zone, average nutrient anomaly at $120 \mathrm{~m}$, nutrient flux calculated as $\mu N^{\prime}$ at $120 \mathrm{~m}$. The black dots show the most extreme values of nutrient anomaly. . . . . . . . . . . . . 176

C-4 Scatter plot of all observations of the estimated nutrient flux $\left(\mu N^{\prime}\right)$ as a function of carbon-specific growth rate $(\mu) \ldots \ldots . \ldots 177$

D-1 PV probability density functions on model day 50 in the mixed layer (blue line), on outcropping isopycnals, but below the mixed layer (green line), and of water that subducted from the mixed layer during an offline tracer experiment that started on model day 43.75 (orange line). . . . . . . . . . . . . . 180

D-2 Volume of the mixed layer through the analysis period. The mixed layer volume is fairly constant throughout the analysis period, indicating that there is little restratification. . . . . . . . . . . . . . . . . . 180

E-1 POC estimated from $b_{b p}(700)$ on the ecoCTD using the conversion relationship from Mayot et al. (2017) plotted against AOU for CLP18 and CLP19. Each point is a sample and is colored with the sample depth. . . . . . . . . 181

E-2 Correlation between ship-board CTD $c_{p}$ and ecoCTD $b_{b p}(700)$ from the calibration cast on the CLP18 cruise. The beam c values should not be negative. I think they need to be calibrated against the dark values. . . . . . . . . . . . 182

F-1 Spatial distribution of transects as in Figure 5-1 but with the background of satellite sea surface temperature. The transect from IRENE is pink, the transects from CLP18 are blue, and the transects from CLP19 are green. . . . 184

F-2 Spatial distribution of transects as in Figure 5-1 but with the background of WMOP model salinity and sea surface height from May 31, 2018. The transect from IRENE is pink, the transects from CLP18 are blue, and the transects from CLP19 are green. . . . . . . . . . . . . . . 185

F-3 Distribution of forward scattering of each taxonomic group from flow cytometry in the filaments and background below $100 \mathrm{~m}$ in each region during CLP19.185

F-4 From left to right IRENE, CLP18, CLP19. The upper panels show chlorophyll concentration plotted against stratification. The lower panels show example chlorophyll profiles. The background of the panels shows the stratification on that profile. Each of these profiles has a secondary chlorophyll maximum. . . 186 
F-5 Combined analysis of flow cytometry and amplicon sequencing. The top row is the eukaryotic abundance from flow cytometry. The second row is the eukaryotic cell size from flow cytometry. The third row shows the relative abundance of plastids (left legend) with the samples arranged in the same order as the upper panels. The bottom row shows the relative abundance of Prasinophytes from amplicon sequencing. The left column shows only samples where I identified a population of small picoeukaryotes in the flow cytometry cytograms. The right column shows all samples from CLP19 with the samples in the left column colored in red in the second row. . . . . . . . . . . . . 186

F-6 POC export during CLP18. (top) Probability density functions of POC concentration within the intrusions (green) and POC concentration randomly sampled with the same depth distribution as the intrusions but outside the intrusions. The dashed line shows the geometric mean of the POC distribution in the gyre intrusion and background. The dotted line shows the mean of the POC distribution in the eddy intrusion and background. The grey shading shows the $95 \%$ confidence interval. These averages are also shown in the panel below. (bottom left) Average POC profile in the gyre (dashed) and eddy (dotted). The scatter points show the intrusion POC concentrations at each depth below $100 \mathrm{~m}$. The darker points are in the eddy and the lighter points are in the gyre. (right) Depth distribution of intrusion samples below

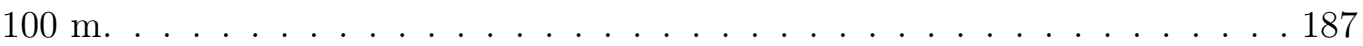

F-7 (upper) Average profiles of photosynthetic cell counts in each water mass from CLP19. (lower) Average temperature profiles for each water mass. . . . . . . 188

F-8 Ordination of cyanobacteria and viridiplantae. (top) Non-metric multidimensional scaling (NMDS) of the Bray-Curtis distance between samples. The sample feature is shown with the color of the point and the research cruise is shown in the shape of the point. (bottom) The first NMDS axis is plotted against the sample depth. . . . . . . . . . . . . . . . . . . . . . . . 189

F-9 NMDS ordination of Bray-Curtis dissimilarity between samples computed on ASVs (excluding plastids and cyanobacteria). The sample water mass assigned based on T-S characteristics is shown with the color of the point and the research cruise is shown in the shape of the point. NMDS stress $=0.071$ with 3 axes. . . . . . . . . . . . . . . . . . . . . . 190 


\section{List of Tables}

2.1 Values used for initial buoyancy gradients and isopycnal slopes . . . . . . . 47

2.2 Parameter values used in the biological model . . . . . . . . . . . . . . . . 50

5.1 Overview of research cruises. Average MLD is the average mixed layer depth in the CTD cast density profile. . . . . . . . . . . . . . . . . 116

5.2 POC concentration $(\mu \mathrm{mol} / \mathrm{kg})$ and $\mathrm{AOU}(\mu \mathrm{mol} / \mathrm{kg})$ in intrusions below $100 \mathrm{~m}$ and outside the intrusions ("sampled background"). The intervals show the bootstrapped $95 \%$ confidence interval of the geometric mean of each category (1000 iterations). The concentration outside the intrusions is calculated by averaging the POC concentration from a random sample of points with the sample depth distribution as the intrusion samples. The variation in the geometric mean and the confidence intervals among random samples from the background is less than $0.001 \mu \mathrm{mol} / \mathrm{kg}$. The location is the research cruise and region. CLP19 WAG is transects C1, C2, and C5 as well as the casts between $\mathrm{C} 1$ and $\mathrm{C} 2$ where only nutrients are sampled. CLP19 eddy is transects $\mathrm{C} 3$ and $\mathrm{C} 4$ and the transect near $\mathrm{C} 3$ with only nutrients sampled. CLP18 is transects B1 and B2. The t-test p value that the geometric mean in the intrusions is significantly different from the sampled background is less than 0.001 in all cases. . . . . . . . . . . . . . . . . . . 125 


\section{Chapter 1}

\section{Introduction}

Motion of water in the vertical is a fundamental part of ocean circulation at all scales. At the largest scales, the rate of the global thermohaline circulation is thought to be set by the regions of upwelling and downwelling at high latitudes (Lozier et al., 2017) and by weak $\left(\mathcal{O}\left(10^{-7}\right) \mathrm{m} / \mathrm{s}\right)$ upwelling in the ocean interior (Stommel and Arons, 1959). At basin scales, the vertical velocity is relatively weak due to the constraints of planetary rotation. Much of the classical work on ocean circulation has been carried out in a quasi-2D framework using a finite number of layers which reduces the complexity of motion in the third dimension, depth (Salmon, 1994). At the smallest scales (meters), the fluid motion is turbulent and fully three-dimensional. In between, there is a wide range of processes that generate vertical velocity including convection, wind-driven Ekman dynamics, tides, internal waves, and mesoscale and submesoscale fronts and eddies. Vertical motions that range from modulation of density structure by climate oscillations to seasonal deepening of the mixed layer to mesoscale eddies with lifetimes of months to boundary layer turbulence with timescales of minutes have been observed to affect biogeochemical fluxes. The spatial and temporal scales of ocean dynamics are linked with larger scale processes also generally operating on slower timescales (Stommel, 1963).

At intermediate spatial scales, the mesoscale and submesoscale, one of the major motivations for understanding the characteristics of ocean vertical velocity is the potential role of vertical advection processes in controlling primary production, carbon export, and heat

fluxes in the upper ocean and exchange between the surface ocean and interior (Mahadevan, 


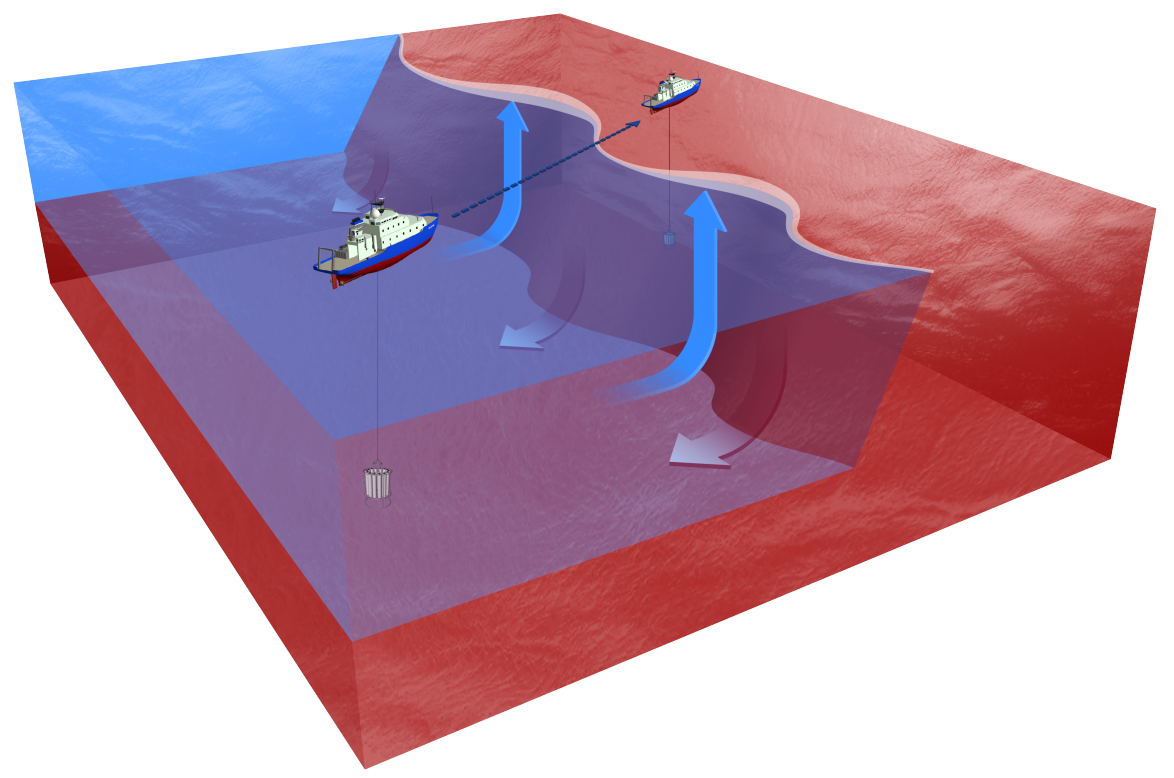

Figure 1-1: Schematic illustration of a front. A front is the confluence between two water masses that have distinct properties, here indicated by the distinct colors. As the front meanders and develops other instabilities, upwelling and downwelling is generated at the front (arrows). This upwelling and downwelling transports heat and biogeochemical tracers. This dissertation uses data collected from ship-board surveys across fronts. One of the tools is a CTD rosette, which is shown in the water below the ships. The rosette has Niskin bottles to collect seawater from below the surface. The ships and the front are not scaled proportionally. Figure created by Eric Taylor, WHOI graphics for Freilich (2018).

2016; McGillicuddy, 2016a). The vast majority of oceanic primary production, about 99\%, is generated by microscopic organisms. These organisms, called phytoplankton, are carried along with water parcels because those that are motile swim slower than ocean currents. If we were to follow along with a phytoplankton cell of the course of the day, we would experience fluctuations in light throughout the day as we are carried in the ocean turbulence instead of the predictable increase in light at sunrise and decrease at sunset to which we are accustomed on land. These turbulent motions affect the rate and magnitude of productivity and of carbon fixation because photosynthesis depends on light. Light available for photosynthesis decays roughly exponentially with depth in seawater. Photosynthesis therefore only takes place within the upper part of the water column, called the euphotic zone. This 
vertical structuring in primary production also affects nutrient distributions. Over most of the ocean, nutrients such as nitrate and phosphate are at high concentrations at depth and low concentrations in the surface ocean (Omand and Mahadevan, 2015) because phytoplankton take up these nutrients in the surface ocean where there is enough light energy to grow and are remineralized by bacteria at depth. Vertical motion that advects nutrients from depth into the euphotic zone across a large nutrient gradient can therefore promote production (McGillicuddy et al., 1998b; Johnson et al., 2010). Some phytoplankton can respond quickly to being transported into a higher light environment and increase their growth rate, others are not able to respond to fast fluctuations and some are even damaged by a fluctuating light environment (Rodriguez et al., 2001; Wyatt, 2014).

Some of the early literature that pointed to a link between vertical advection by eddies and primary production focused on a mesoscale eddies, which generate excursions of density surfaces by tens of meters and persist for months (McGillicuddy, 2016a) and linear internal waves. However, processes that are on smaller scales than the mesoscale, the submesoscale, are associated with faster vertical velocity, reaching $100 \mathrm{~m}$ /day (McWilliams, 1985; Mahadevan and Tandon, 2006; Capet et al., 2008; McWilliams, 2016), and have a differential effect on phytoplankton productivity and diversity (Mahadevan, 2016). The first part of this dissertation focuses on the role of submesoscale dynamics in altering upward fluxes of nutrients and the resulting primary production.

The submesoscale is between the scales where rotation constrains the dynamics and three dimensional turbulence that is unaffected by rotation. At the submesoscale, rotation is still important, but no longer the only leading order process. Another way to state this definition of the submesoscale is through a non-dimensional number, the Rossby number which is defined as Ro $\equiv \frac{\mathrm{U}}{f \mathrm{~L}}$ where $\mathrm{U}$ is the velocity scale, $\mathrm{L}$ is the length scale, and $f$ is the Coriolis parameter. At the submesoscale, the Rossby number is order 1. At a given latitude (constant $f$ ), Ro can be $\mathcal{O}(1)$ if the inverse timescale of the currents $\frac{\mathrm{U}}{\mathrm{L}}$ is $\mathcal{O}(f)$ (the eddy scale $\mathrm{L}$ is comparable in size to an inertial circle). This can be achieved with relatively fast currents, with variation on relatively small length scales, or both. In the mid-latitudes where $f \sim 10^{-4}$, the submesoscale length scale is typically $1-10 \mathrm{~km}\left(10^{3}-10^{4} \mathrm{~m}\right)$ and the velocity is in the range of $0.1-1 \mathrm{~m} / \mathrm{s}$. Due to the relatively small scales of variation of the 
horizontal velocity, submesoscale dynamics are also associated with relatively large vertical velocity. The vertical velocity $(w)$ is related to the divergence of the horizontal velocity $(\mathbf{u}=(u, v))$ as $\nabla \cdot \mathbf{u}=-w_{z}$. Assuming quasigeostrophic dynamics, the vertical velocity scales as $w \sim \operatorname{Ro} \frac{\mathrm{H}}{\mathrm{L}} \mathrm{U}$ where $\mathrm{H}$ is the scale of variations in depth. At scales larger than the submesoscale, the mesoscale, the vertical velocity is weaker than at the submesoscale due to both the small aspect ratio $\frac{\mathrm{H}}{\mathrm{L}}$ and small Ro. At the submesoscale, Ro $\sim \mathcal{O}(1)$ and the vertical velocity is only smaller than the horizontal velocity by $\mathcal{O}\left(\frac{\mathrm{H}}{\mathrm{L}}\right)$, which is larger at fronts by still usually less than $10^{-2}$. Submesoscale dynamics are distinguished from internal waves, which have similar magnitudes of vertical velocity and can have similar length scales, due to the large submesoscale vorticity in addition to the large divergence (the criteria for submesoscale is sometimes written as Ro $=\frac{\zeta}{f} \geq 1$ ), which makes submesoscale dynamics more non-linear than waves.

One of the characteristics of near surface submesoscale vertical velocity is that the vertical velocity is skewed with larger magnitude downward than upward vertical velocities (Shcherbina et al., 2013). In a mass conserving system the average vertical velocity at any given depth level is zero, so the larger magnitude submesoscale downward vertical velocity is concentrated in a smaller area compared to the upward vertical velocity, which is more diffuse over a larger area (Ruiz et al., 2019). At the submesoscale, relatively rapid downward vertical motion (subduction) could have implications for the export of carbon, oxygen, and nutrients (Stukel et al., 2017; Barth et al., 2002; Ruiz et al., 2009; Omand et al., 2015; Erickson et al., 2016). The second part of this dissertation focuses on the processes driving subduction at fronts, the role of submesoscale dynamics in subduction, and the biogeochemical and ecological consequences of subduction.

One theme of this dissertation is advancing understanding of the interaction between and separation of mesoscale and submesoscale dynamics. The patterns and magnitude of vertical velocity are sensitive to model resolution. When smaller scale buoyancy and velocity gradients are resolved by running models with smaller scale grid spacing (higher resolution), the vertical velocity becomes larger (Mahadevan and Tandon, 2006; Capet et al., 2008). This can help to separate mesoscale (low resolution) from submesoscale (high resolution) dynamics. In simulations that include many interacting processes, and in observations, there is 
not a clear break in the dynamics between the mesoscale and submesoscale (Ramachandran et al., 2014). As Ro becomes larger, the flow becomes more non-linear, but there is no clear separation between mesoscale and submesoscale except in the case of instabilities that are classified as submesoscale such as symmetric instability and mixed layer baroclinic instability (Thomas and Joyce, 2010; Thomas et al., 2013; Fox-Kemper et al., 2008). As model resolution is increased, gradients sharpen, new features develop, and in many cases mesoscale eddy fields become stronger (Lévy et al., 2001). In addition, resolving submesoscale dynamics can result in alterations to the overall stratification and nutrient distribution, meaning that it can be difficult to isolate the direct effect of submesoscale dynamics on one part of a complex system (Lévy et al., 2012c). Complicating the issue further, submesoscale features are generated by mesoscale straining, although their subsequent evolution may not have a mesoscale characteristic (Barkan et al., 2019). These dynamics are important for understanding the energy sources of the submesoscale. Energy at the submesoscale has been shown to cascade upscale to the mesoscale or downscale towards dissipation (Qiu et al., 2014).

The second major theme of this dissertation is the effect of submesoscale vertical motion on new production and carbon export. When we consider the effects of vertical velocity on biogeochemical fluxes, the problem is not just the vertical velocity at any given instant, but also the integrated effect of vertical motion on the distribution of biogeochemical tracers. Tracer distributions show the integrated effect of ocean circulations. For example, a wave might carry a water parcel up and then down again over the course of a day. A passive tracer carried along with that water parcel would show no net displacement in the vertical because it integrates over the upward and downward motions. When considering the problem of biogeochemical fluxes, we need to assess the net effect of the vertical motion on the biogeochemical quantity over the relevant timescale and, additionally, the ecological response to that flux.

We make headway towards fundamental understanding using simplified models. One of the key tools that we use is process study modeling. We incorporate certain aspects of observations into a non-hydrostatic ocean model which allows us to idealize the oceanographic situation and gain deep understanding of certain processes without the need to account for the full complexity of all possible physical forcing (Mahadevan et al., 1996a). Our biological 
models also distill complex processes down to their fundamental features. The biological models used here tend more towards abstracted than detailed. The goal is to advance understanding but not to make the most complete model. Each of the analyses is grounded in observations and we are careful to only include model processes for which we have an observational constraint.

Despite the importance of vertical velocity, the scientific tools to measure vertical motion and three dimensional transport have been available relatively recently and are still in development. Most vertical velocity is at the noise limit of acoustic Doppler current profilers (ADCPs), a common tool for measuring ocean velocity, and other direct measurement techniques (Frajka-Williams et al., 2011). Lagrangian floats can accurately measure vertical transport, but the measurements are restricted to a small area and limited time period (D'Asaro, 2003).

Vertical velocity has a profound effect on primary production by phytoplankton. Some of the early observational evidence for the influence of fine-scale physical dynamics on primary production came from satellites, which can measure the light field at the sea surface over large areas (Eslinger and Iverson, 1986). The satellite observations can be used to estimate chlorophyll concentrations (a proxy for phytoplankton concentrations) (Figure 1-2) and, to a certain extent, community composition (Alvain et al., 2008). These observations reveal that chlorophyll at the ocean surface is patchy with spatial structure down to the smallest scales of observation $(1 \mathrm{~km})$ that resembles the characteristics of lateral stirring by ocean currents (Abraham, 1998; Mahadevan and Campbell, 2002). In addition, the community composition shows signatures of stirring water masses laterally at the sea surface (d'Ovidio et al., 2010). However, in addition to this lateral stirring there are also observations of enhancement of productivity at small scale, suggesting that understanding small scale physical processes, on the scale of ones to tens of kilometers, is important for understanding biogeochemical cycling (Mahadevan and Archer, 2000).

In three dimensions, we do not currently have global high resolution autonomous observations of tracer distributions, but there is ubiquitous evidence of tracer transport at more local scales from ship-based surveys and autonomous platforms (Washburn et al., 1991; Stanley et al., 2017). Potential vorticity (PV) and spice are two commonly used tracers in the 


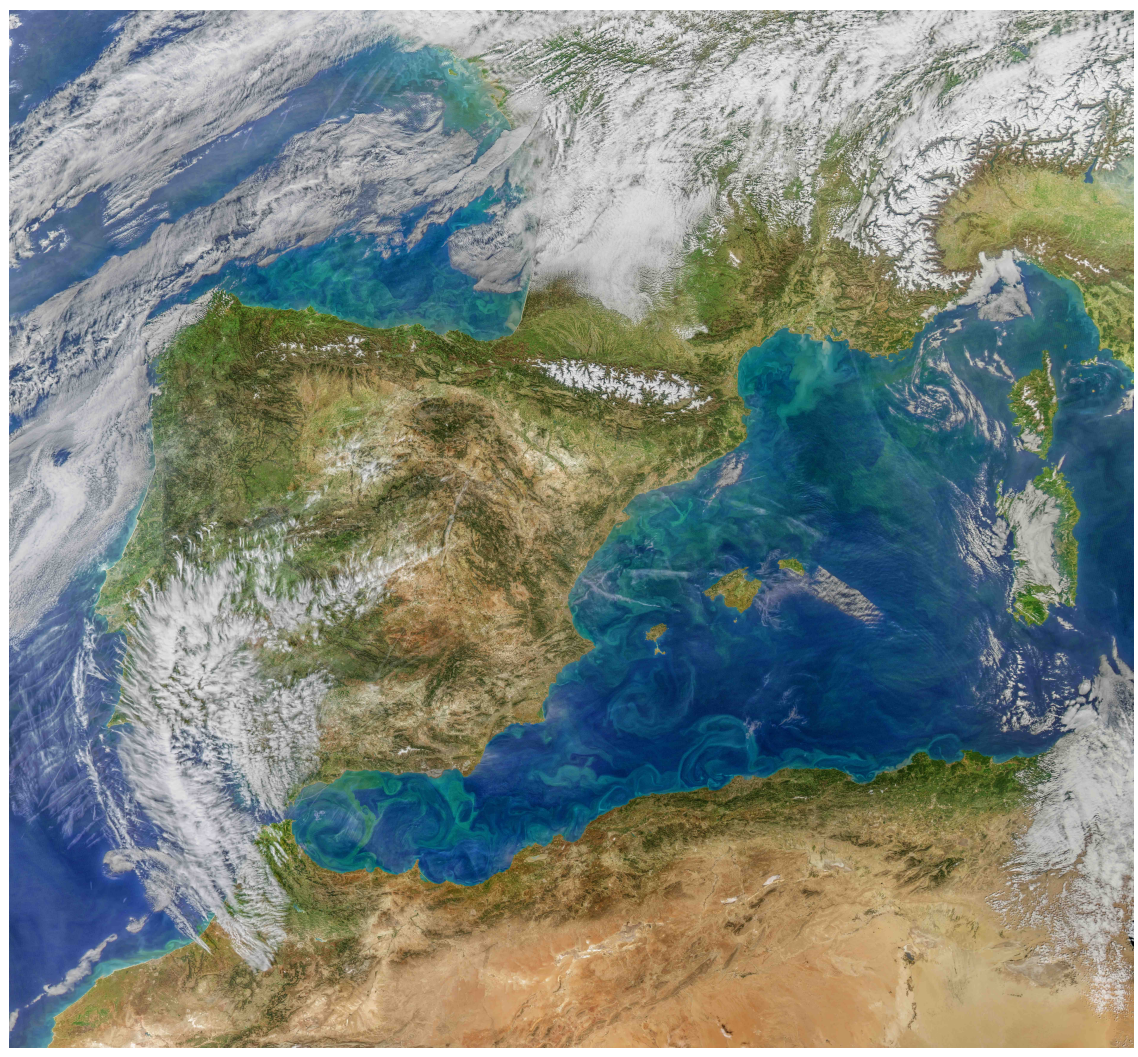

Figure 1-2: MODIS satellite observation of chlorophyll on March 11, 2020 in the Western Mediterranean Sea. The chlorophyll distributions show influence of upwelling and stirring.

physical oceanography literature. Spice is the combination of temperature and salinity that result in the same density and serves as a passive tracer on a density surface (McDougall et al., 2021). Observations of the variance of these tracers across scales is used to assess consistency between observations and theoretical predictions of tracer distributions. Both spice and PV are conserved quantities with sources and sinks (heat, friction (PV), and freshwater (spice)) that are largest at the sea surface. In addition, different water masses will have different temperature and salinity characteristics, with some being fresher and cooler (mintier) and others being warmer and saltier (spicier). This leads to naturally occurring gradients in these quantities. When ocean circulation acts on these gradients, they become tracers of that motion. Observations of low PV water in the interior provide evidence of transport of surface waters (Spall, 1995; Pallàs-Sanz et al., 2010; Archer et al., 2020).

Geochemical, biogeochemical, and biological tracers often have strong vertical gradients, as described above, so they can serve as natural tracers of vertical motion. However, these 
tracers are also modified by reactions that may be depth dependent (such as nutrient uptake) and which also have an inherent timescale that may be useful as additional information. Due to the inherent timescale associated with the the reactions of these tracers, observations of the distributions of biological and chemical tracers can also be used to estimate the rates of vertical motion, with varying accuracy (Kadko et al., 1991; Washburn et al., 1991; PallàsSanz et al., 2010; Oka et al., 2009; Thomas and Joyce, 2010; Poje et al., 2014). In some experiments, artificial dyes are used as tracers of ocean circulation (Ledwell et al., 1993; Shcherbina et al., 2015; Wenegrat et al., 2020). The artificial dye distribution reveals the advection and mixing over the known time interval from the time of release to the time of observation.

The distributions of naturally occurring tracers provides evidence of stirring by ocean dynamics. Work on spice distributions has shown that submesoscale processes, in particular subduction, frontogenesis, large shear, and internal waves, are needed to account for the distribution of passive tracers subsurface (Ferrari and Rudnick, 2000; Kunze et al., 2015; Spiro Jaeger et al., 2020). Most of these studies focus on lateral stirring on isopycnal surfaces. The theoretical framework for studying lateral stirring on isopycnal surfaces is the simplification of ocean dynamics in terms of $2 \mathrm{D}$ theories. However, isopycnal surfaces have height variations and there is therefore a vertical component to the stirring of tracers that interacts with biological processes to enhance primary production and carbon export (Figure 1-3).

Chapter 2 addresses the extent to which vertical transport is due to motion on sloping isopycnal surfaces. In the absence of direct measurements of vertical velocity, previous work has inferred vertical fluxes by observing variations in height of the sea surface at the mesoscale and variations in height of constant nutrient surfaces. We propose an alternative approach by deriving a decomposition of the vertical velocity into a part that is due to changes in the height of a density surface $\left(w_{\text {uplift }}\right)$ and a part that is due to motions along a sloping density surface $\left(w_{i s o}\right)$. Application of the decomposition to a series of process study models based on the Sargasso Sea eddy field that span a range of eddy energy confirms the importance of the along isopycnal vertical velocity. This work proposes that one of the characteristics of submesoscale vertical motion is that $w_{\text {iso }}$ and $w_{\text {uplift }}$ make similar 


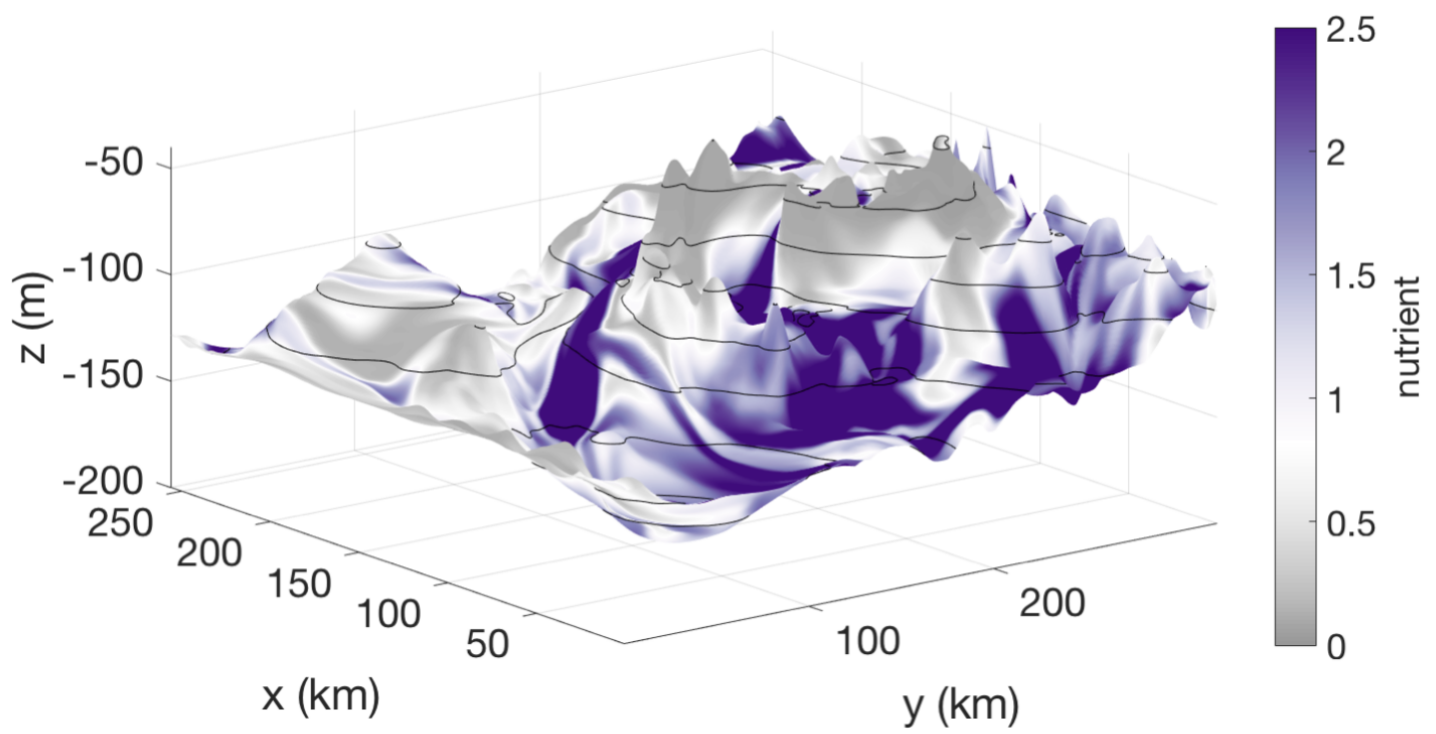

Figure 1-3: Nutrient as a tracer of fluid motions on a constant density (isopycnal) surface. The nutrient concentration is affected by both biological reactions and physical transport. The surface has variations in height such that motion on this surface could have a vertical component. This figure was generated from the model analyzed in chapters 2 and 3

magnitude contributions to the total vertical velocity while at larger scales $w_{\text {uplift }}$ is the dominant type of vertical motion. This distinction in the nature of the vertical velocity has implications for passive tracer transport. A scaling relationship that is valid across the model resolutions connects this decomposition to frontal dynamics and allows us to highlight other regions globally where $w_{\text {iso }}$ might be important.

When we turn to the issue of addressing the impact of these different vertical velocity processes on the vertical nutrient flux, we find that the vertical nutrient flux is sensitive to the biological model in addition to the physics. In previous work, resolving submesoscale processes has been found to increase the depth of the nutricline relative to low resolution simulations by increasing the depth of the mixed layer and the amount of productivity (Lathuiliere et al., 2010; Gruber et al., 2011; Lévy et al., 2012b). The flux cannot be quantified without considering the distribution and sources and sinks of the tracer that is being fluxed. In chapter 3 we demonstrate using a theoretical derivation, process study model, and observations from the North Pacific subtropical gyre that the vertical nutrient flux depends on the biological timescale. Even with a linear biological model, the magnitude 
of the biological flux is a nonlinear function of the biological rate. We investigate time integrated processes on water parcels using Lagrangian methods. In Lagrangian analysis, we follow the $3 \mathrm{D}$ motion of water parcels

One approach for addressing the integrated transport over time is to use Lagrangian methods where we follow an individual water parcel and track tracers and dynamics on that water parcel. In a Lagrangian frame, the rate of change in the position of a water parcel (labeled $n$ ) is the velocity

$$
\frac{\mathrm{d} \mathbf{x}^{(\mathbf{n})}}{\mathrm{d} t}=\mathbf{u}^{(\mathbf{n})}(\mathbf{x}(\mathbf{t}), \mathbf{t})
$$

The rate of change of any property $(c)$ following a water parcel is given by its material derivative and modification by sources and sinks

$$
\frac{\mathrm{D} c}{\mathrm{D} t}=\text { sources-sinks. }
$$

The velocity used in the material derivative in equation 1.2 is the velocity determined by following trajectories as in equation 1.1. Practically, we do Lagrangian analysis using "particles" in a model. Lagrangian analysis methods are used throughout this dissertation. In terms of the dynamics, the time mean Eulerian fields may not necessarily be the fields that contribute to tracer flux. The classic example of an Eulerian circulation that does not appear in Lagrangian trajectories is the atmospheric Ferrel cell (Plumb and Mahlman, 1987). Doddridge et al. (2016) suggest that eddying circulation cancels a large part of the Ekman downwelling in subtropical gyres, which would mean that the nutricline is shallower than expected from the wind-driven circulation alone.

The vertical velocity decomposition introduced in chapter 2 can only be carried out below the mixed layer but there is along isopycnal motion at the mixed layer as well. The mixed layer is the interface between the atmosphere and ocean and exchange across this interface is crucially important for exchange between the surface and the interior. In chapter 4, we focus on a situation with a deep reaching front with density surfaces that outcrop at the sea surface and run a process study model based on the Almería-Oran front in the Western Mediterranean Sea. At the Almería-Oran front, tracer distributions revealed that 
there was exchange from the mixed layer into the interior. In a model, we observe strong vertical velocities in the mixed layer, however water parcels do not necessarily cross the base of the mixed layer. To identify the water parcels that cross the mixed layer base and analyze their dynamics, we perform a Lagrangian analysis. We find that there are a wide variety of mesoscale and submesoscale frontogenesis processes that drive subduction across the mixed layer base. This chapter unravels important pathways for exchange between the ocean surface and interior that are not included in global models and result in $100 \mathrm{~m}$ of the mixed layer being subducted over the course of a year.

In chapter 5 we elaborate on the observational evidence for subduction. We use observations of tracer distributions at fronts in the Western Mediterranean Sea to demonstrate that subduction from the surface and from the deep biomass maximum transports carbon, oxygen, and chlorophyll into the interior. We use the biogeochemical tracers to estimate the vertical velocity magnitude. After establishing the subduction is occurring, we examine the relationship between the physical and biological processes by asking what is the composition of the biological material that is being subducted, where and when it is subducted, and what transformations it undergoes as it is being subducted. To answer these questions, we analyze the biological community composition within the frontal region and in the subducted features. This chapter introduces new mechanisms for both carbon export and the vertical structure of community composition.

Chapter 6 summarizes the conclusions and offers an outlook on future research on submesoscale biophysical interactions. 


\title{
Chapter 2
}

\section{Decomposition of vertical velocity for}

\section{nutrient transport in the upper ocean}

\begin{abstract}
Within the pycnocline, where diapycnal mixing is negligible, both the vertical movement (uplift) of isopycnal surfaces and upward motion along sloping isopycnals supply nutrients to the euphotic layer, but the relative importance of each of these mechanisms is unknown. We present a method for decomposing vertical velocity, $w$, into two components in a Lagrangian frame: Vertical velocity along sloping isopycnal surfaces, $w_{\text {iso }}$, and the adiabatic vertical velocity of isopycnal surfaces, $w_{\text {uplift }}$. We show that $w_{\text {iso }} \sim \frac{M^{2} L}{N^{2} H} w$, where $\frac{M^{2}}{N^{2}}$ is the isopycnal slope and $\frac{H}{L}$ is the geometric aspect ratio of the flow, and that $w_{\text {iso }}$ accounts for $10-25 \%$ of the total vertical velocity, $w$, for isopycnal slopes representative of the mid-latitude pycnocline. We perform the decomposition of $w$ in a process study model of a mid-latitude eddying flow field generated with a range of isopycnal slopes. A spectral decomposition of the velocity components shows that while $w_{\text {uplift }}$ is the largest contributor to vertical velocity, $w_{\text {iso }}$ is of comparable magnitude at horizontal scales less than about $10 \mathrm{~km}$, i.e. at submesoscales. Increasing the horizontal grid resolution of models is known to increase vertical velocity; this increase is disproportionately due to better resolution of $w_{i s o}$, as is shown here by comparing $1 \mathrm{~km}$ and $4 \mathrm{~km}$ resolution model runs. Along-isopycnal vertical transport can be an important contributor to the vertical flux of tracers, including oxygen, nutrients, and chlorophyll, although we find weak covariance between vertical velocity and nutrient anomaly in our model. ${ }^{1}$
\end{abstract}

\footnotetext{
${ }^{1}$ This chapter is published as Freilich, M and Mahadevan, A (2019). Decomposition of vertical velocity for nutrient transport in the upper ocean. Journal of Physical Oceanography, 49(6):1561-1575. (C) American Meteorological Society. Used with permission.
} 


\subsection{Introduction}

The ocean's vertical velocity encompasses multiple processes over a range of time and space scales, from the turbulent scale to global overturning scale. Vertical velocity is difficult to measure or estimate accurately as it is $3-4$ orders of magnitude smaller than the mesoscale horizontal velocity, and is noisy and close to the detection limit of most sensors. Though poorly understood, vertical velocity contributes to the vertical transport of tracers that are important from a biogeochemical perspective. Several properties like oxygen, dissolved and particulate forms of carbon, and nutrients like nitrate, phosphate and silicate, exhibit strong gradients in the vertical due to depth-dependent sources and sinks, and weak vertical mixing. Their upward and downward transport toward or away-from the sunlit surface and air-sea interface has implications for the productivity of phytoplankton, the biological pump of carbon, the exchange of atmospheric gases, and the ventilation of the oxygen-deficient ocean interior (Calil and Richards, 2010; Lévy et al., 2012a; Thomsen et al., 2016; Balwada et al., 2018).

Phytoplankton primary production depends on both light, which attenuates exponen-

tially with depth, and nutrients, which are abundant at depth and depleted in the surface sunlit (euphotic) layers. In nutrient-limited (oligotrophic) regions, phytoplankton depend on physical mechanisms to transport nutrients from depth into the sunlit euphotic zone. But, the mechanisms for vertical transport of nutrients have long been debated. Beneath the surface mixed layer, and in the pycnocline, diapycnal nutrient flux is weak, and the flow is to a good approximation adiabatic (isopycnal following) and incompressible (Lewis et al., 1986; McGillicuddy et al., 1998b). In the stratified pycnocline, the vertical velocity is largely a combination of vertical motion of isopycnal surfaces (isopycnal uplift) and vertical motion along sloping isopycnal surfaces. Uncovering the mechanisms for nitrate transport in this environment could help reconcile a paradox in subtropical gyres, where carbon-based measurements of phytoplankton productivity are higher than nitrogen-based estimates, suggesting a missing nutrient source (Zhou et al., 2013).

On large scales, dissolved nitrate increases with density and depth in the ocean; the variance of nitrate is lower on isopycnal surfaces than on depth surfaces (Omand and Ma- 
hadevan, 2015). Deeper isopycnal surfaces, which have higher nitrate, are also iso-nutrient surfaces. Since measured diapycnal diffusion is too weak to supply the nutrients required for phytoplankton production (Caldwell, 1983; Ledwell et al., 1993), eddies and ocean fronts are thought to play an important role for the vertical nutrient flux. One mechanism for nutrient supply is the heaving of isopycnal surfaces upwards into the euphotic zone, such as by mesoscale eddies. This mechanism, referred to here as "isopycnal uplift", and previously termed "eddy pumping" (McGillicuddy et al., 1998b; Charria et al., 2003; McGillicuddy, 2016a), is hypothesized to be of primary importance. While originally formulated at the scale of mesoscale eddies and modeled using quasigeostrophic equations (McGillicuddy et al., 1998b), isopycnal uplift can also operate at smaller scales than the Rossby radius in quasibalanced flows (Johnson et al., 2010; Ascani et al., 2013). The rate of uplift compared to the rate of nutrient uptake and the non-linearity of the eddy determine the total nutrient transport into the euphotic zone due to isopycnal uplift (Martin and Pondaven, 2003). Internal waves and tides also contribute to the vertical movement of isopycnals and can affect phytoplankton productivity (Denman and Gargett, 1983; Flierl and McGillicuddy, 2002), but can be separated from quasi-balanced motions associated with mesoscale eddies (Flierl and McGillicuddy, 2002). They are not considered in this study, as their time scales are shorter than the typical timescales of phytoplankton growth in oligotrophic regions.

An alternate hypothesis is that transport along sloping isopycnal surfaces supplies nutrients to the euphotic zone. Though geostrophic flow on isopycnal surfaces is mostly along pressure contours, deviations from geostrophy allow the flow to transport nutrient up- or down-slope along sloping isopycnals. The degree of vertical flow is associated with the loss of geostrophic balance, and hence associated with $O(1)$ Rossby number and submesoscale dynamics that is intensified in the vicinity of strongly sloping isopycnals or fronts and described by primitive equation models (Mahadevan and Archer, 2000). Since the uptake of nutrients on an isopycnal is depth dependent, gradients in nitrate are generated on sloping isopycnals and facilitate down-gradient (up-slope) nutrient transport into the euphotic layer. Recent observational and modeling studies have suggested that episodic nutrient input associated with submesoscale processes could be the source of "missing" nutrients in oligotrophic regimes (Calil and Richards, 2010; Ascani et al., 2013). 
Much of the early work that demonstrated the importance of submesoscale physics for biogeochemical tracer flux used model simulations with progressively finer grid resolution in order to resolve smaller-scale physical processes. These studies found that productivity can be increased by resolving submesoscale physics (Mahadevan and Archer, 2000; Lévy et al., 2001). Subsequent work has shown that increasing model resolution may not necessarily enhance phytoplankton productivity, since, on long timescales, increased vertical transfer of nutrients can result in a deeper nutricline (Lévy et al., 2012b) and submesoscale vertical motion may also subduct unconsumed nutrients or phytoplankton out of the euphotic zone, particularly in upwelling regions of the ocean (Gruber et al., 2011; Lathuiliere et al., 2010).

In quasigeostrophic turbulence, the vertical velocity, $w$, scales with the aspect ratio (of depth $\mathrm{H}$ to length $\mathrm{L}$ scale) and the Rossby number, Ro, as $w \sim R o \frac{\mathrm{H}}{\mathrm{L}} U$, and is therefore, orders of magnitude smaller than the horizontal velocity, $U$. However, in dynamically unbalanced or submesoscale flows, velocities of up to 100 meters per day have been observed (D'Asaro, 2001; D'Asaro et al., 2018) and modeled (Mahadevan and Tandon, 2006; Capet et al., 2008; Klein and Lapeyre, 2009) where $R o=O(1)$. While mesoscale motion is important for phytoplankton productivity, submesoscale motion could be equally important due to the much shorter timescales of vertical nutrient transport that are better matched with the phytoplankton growth rate (Mahadevan, 2016).

Isopycnal uplift and along-isopycnal transport may occur in conjunction. The localized uplift of an isopycnal layer into the euphotic layer is associated with a concurrent squeezing and spreading-apart of isopycnals to accommodate the motion if the motion is baroclinic. This generates a divergence/convergence of the flow along-isopycnal surfaces and thereby along-isopycnal vertical motion. A water parcel uplifted into the euphotic layer on an isopycnal will be rapidly depleted of nutrients, but nutrients must be resupplied along the sloping isopycnal surface to maintain biological productivity, especially if mesoscale eddies continue to be productive for weeks. Advection along isopycnal surfaces is known to be important on the scale of an ocean basin (Palter et al., 2005; Pasquero, 2005; Oschlies, 2002), but it also plays a role in resupplying mesoscale features, which has not yet been quantified .

A mechanistic decomposition of the uplift and along-isopycnal vertical motions would be beneficial in improving our understanding of these processes and their representation 
in numerical models. It is not clear whether these kinds of vertical motion operate at different scales or in different dynamical regimes, given that isopycnal uplift can also occur on scales much smaller than the internal Rossby radius and that along-isopycnal transport can resupply nutrients to mesoscale eddies. A spectral analysis of each individual kind of motion could then reveal the scales at which each is most efficient. Furthermore, identifying their contributions to the transport of nutrients in the ocean will enable us to better quantify the physical nutrient supply. In the longer term, this will also help us design strategies to measure the vertical flux of nutrients or other biological properties in the ocean in addition to improving model parameterizations.

We begin, in Section 2.2, by presenting a method for decomposing the vertical velocity $w$ into two components, isopycnal uplift $w_{\text {uplift }}$ (which could act upward or downward), and vertical motion along-isopycnal surfaces $w_{i s o}$. The subtlety is that these motions are so convolved that they are separable only in the Lagrangian frame, and furthermore, could occur in opposite directions with cancellation between the two. In Section 2.22.2.2, we derive a scaling estimate for $w_{i s o}$ as a fraction of the total vertical velocity $w$. In Section 2.3, we describe a setup for the Process Study Ocean Model (PSOM) that we use for simulating the eddy field in an oligotrophic region with horizontal grid resolutions of $4 \mathrm{~km}$ and $1 \mathrm{~km}$. By applying our decomposition to the numerical model output within the pycnocline, we are able to distinguish the contribution of each vertical velocity component. We couple the physical model to a nutrient-phytoplankton model and calculate the vertical nutrient flux and resulting phytoplankton production associated with each of the vertical velocity components. Wavenumber spectra and probability density functions (PDFs) are used to characterize the vertical velocity components. Finally, in Section 2.5, we discuss the scaling for $w_{\text {iso }}$ in the global context, along with limitations and caveats, before concluding in Section 2.6. 


\subsection{Vertical velocity components}

\subsubsection{Decomposition}

Vertical velocity can be decomposed into two components: the movement of isopycnal surfaces $\left(w_{\text {uplift }}\right)$, which generates a vertical flux of density and of passive tracers, and vertical motion along-isopycnal surfaces $\left(w_{\text {iso }}\right)$, which only contributes to the flux of passive tracers. This calculation must be done in a Lagrangian frame in order to separate the horizontal translation of features, such as non-linear eddies, from Lagrangian vertical motion. In the Eulerian frame, the translation of an eddy past a point would mistakenly indicate isopycnal uplift or downdraft, though water parcels circumnavigating an eddy may not experience any vertical motion. In this study, we use the rotational (or non-divergent) horizontal velocity field as the Lagrangian frame. A velocity field which is purely rotational does not have any vertical motion and the rotational component of the flow field accounts for the bulk of the horizontal translation of features. In another setting, a different Lagrangian frame might be a more appropriate choice.

The horizontal velocity can be separated into a rotational (non-divergent) component $\left(\mathbf{u}_{\text {rot }}\right)$ and a divergent component $\left(\mathbf{u}_{\text {div }}\right)$ using a Helmholtz decomposition. In order to calculate the vertical motion along isopycnal surfaces, we assume that the flow is adiabatic (i.e. does not cross isopycnals). If $h(x, y)$ is the height of the isopycnal $(\sigma)$ surface, its horizontal gradient is $\nabla h=\left(h_{x}, h_{y}\right)$ and the angle the isopycnal surface makes with the horizontal $x$-axis and $y$-axis is given as $\left(\Gamma^{x}, \Gamma^{y}\right)=\left(\tan ^{-1}\left(h_{x}\right), \tan ^{-1}\left(h_{y}\right)\right)$.

The vertical component of the along-isopycnal velocity, $w_{\text {iso }}$, is calculated in two steps. In the first step, we project the divergent part $\left(u_{d i v}, v_{d i v}\right)$ of the total horizontal velocity along with the total vertical velocity, $w$, onto the isopycnal surface (Figure 2-1) to obtain the along-isopycnal velocity vector $\mathbf{u}^{\sigma}=\left(u^{\sigma}, v^{\sigma}\right)$ as

$$
\begin{aligned}
& u^{\sigma}=u_{d i v} \cos \left(\Gamma^{x}\right)+w \sin \left(\Gamma^{x}\right)=\cos \left(\Gamma^{x}\right)\left(u_{d i v}+w h_{x}\right) \\
& v^{\sigma}=v_{d i v} \cos \left(\Gamma^{y}\right)+w \sin \left(\Gamma^{y}\right)=\cos \left(\Gamma^{y}\right)\left(v_{d i v}+w h_{y}\right) .
\end{aligned}
$$

In the second step, the vertical component of the along-isopycnal divergent velocity is 


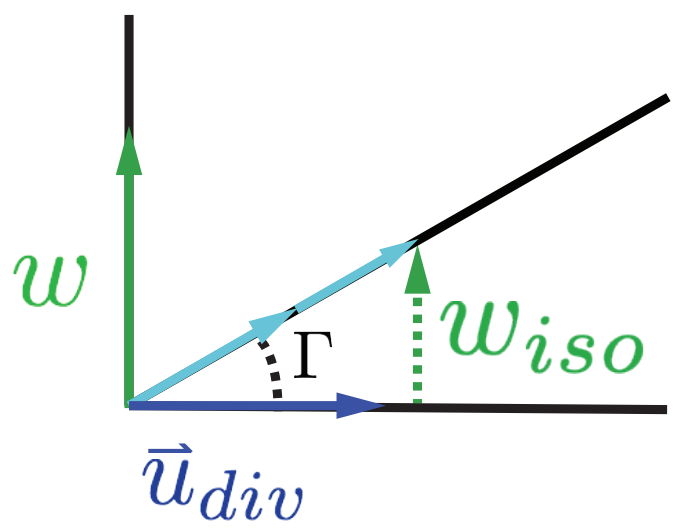

Figure 2-1: Calculation of the vertical component of the along-isopycnal velocity, $w_{i s o}$. $w$ is the total vertical velocity and $u_{d i v}$ is the divergent part of the horizontal velocity. $\Gamma=\tan ^{-1}(|\nabla h|)$. The light blue lines show the projection of $u_{d i v}$ and $w$ onto the isopycnal surface and sum to $u^{\sigma}$ in equation 2.1. The dashed green line is the vertical component of the along-isopycnal velocity.

obtained by projecting the vector $\mathbf{u}^{\sigma}$ in the direction of the steepest slope of the isopycnal surface (which makes an angle $\Gamma$ with the horizontal) and calculating its vertical component as

$$
w_{\text {iso }}=\mathbf{u}^{\sigma} \sin (\Gamma)=\mathbf{u}^{\sigma} \cdot \nabla h \cos (\Gamma) .
$$

Using the relationship $\sin (\Gamma)=|\nabla h| \cos (\Gamma)$, which can also be written as, $\cos ^{2}(\Gamma)=$ $\frac{1}{1+|\nabla h|^{2}}$ and substituting (2.1) into (2.2) gives

$$
w_{\text {iso }}=\frac{\mathbf{u}_{d i v} \cdot \nabla h+w|\nabla h|^{2}}{1+|\nabla h|^{2}} .
$$

The total vertical velocity is

$$
w=\frac{\partial h}{\partial t}+\mathbf{u}_{r o t} \cdot \nabla h+\mathbf{u}_{d i v} \cdot \nabla h .
$$

Substituting (2.4) into (2.3) we find that

$$
w_{i s o}=\mathbf{u}_{d i v} \cdot \nabla h+\frac{|\nabla h|^{2}}{1+|\nabla h|^{2}}\left(\frac{\partial h}{\partial t}+\mathbf{u}_{r o t} \cdot \nabla h\right) .
$$


Since $w=w_{\text {iso }}+w_{\text {uplift }}$,

$$
\begin{array}{r}
w_{\text {uplift }}=w-w_{\text {iso }}=\frac{\partial h}{\partial t}+\mathbf{u}_{r o t} \cdot \nabla h+\mathbf{u}_{d i v} \cdot \nabla h-\mathbf{u}_{d i v} \cdot \nabla h \\
-\frac{|\nabla h|^{2}}{1+|\nabla h|^{2}}\left(\frac{\partial h}{\partial t}+\mathbf{u}_{r o t} \cdot \nabla h\right)
\end{array}
$$

which simplifies to

$$
w_{\text {uplift }}=\frac{1}{1+|\nabla h|^{2}}\left(\frac{\partial h}{\partial t}+\mathbf{u}_{r o t} \cdot \nabla h\right)
$$

Equations (2.5) and (2.7) enable us to diagnose $w_{\text {iso }}$ and $w_{\text {uplift }}$ from the horizontal velocities and the slope and movement of the isopycnal surfaces. A caveat about this decomposition is that $w_{\text {iso }}$ and $w_{\text {uplift }}$ are not mutually independent and there are situations where the two could act in opposite directions. The choice of the Lagrangian frame does not guarantee orthogonality, and admittedly, the divergent component of the velocity $\mathbf{u}_{d i v}$ may also advect isopycnals.

\subsubsection{Scaling relationships}

Scalings can be derived for $w_{\text {iso }}$ to assess its importance in different flow regimes. To begin with, if all of the vertical motion is due to linear gravity waves, nearly all of the motion is isopycnal uplift. In past work on eddy pumping, eddies have been approximated to propagate like linear waves (Martin and Pondaven, 2003). For a linear wave propagating in one dimension at constant speed $c$, the wave (isopycnal) height, $h$, is given by

$$
\frac{\partial h}{\partial t}=-c \frac{\partial h}{\partial x}
$$

Assuming $u=u_{\text {div }}$ and $u_{\text {rot }}=0$, we can evaluate the relative importance of uplift of the layer and vertical movement along the interface using equation (2.5) to estimate

$$
w_{\text {iso }}=u h_{x}+\frac{h_{x}^{2}}{1+h_{x}^{2}} h_{t}=u h_{x}-\frac{h_{x}^{3}}{1+h_{x}^{2}} c
$$


and equation (2.7) to estimate

$$
w_{\text {uplift }}=\frac{1}{1+h_{x}^{2}} h_{t}=\frac{-c h_{x}}{1+h_{x}^{2}}
$$

The ratio between the two vertical velocity components is then

$$
\frac{w_{\text {iso }}}{w_{\text {uplift }}}=-\frac{u}{c}+h_{x}^{2}\left(1-\frac{u}{c}\right) \approx h_{x}^{2} \text { for } \frac{u}{c} \ll 1
$$

Waves moving faster than individual fluid elements, $\frac{u}{c} \ll 1$, are linear. As is commonly assumed, the movement of the fluid elements can be neglected when the slope of the wave $h_{x} \ll 1$ so the along-isopycnal vertical transport is negligible.

In order to derive a more general scaling for the relationship between the along-isopycnal component of the vertical velocity and the total vertical velocity, we assume that the flow is in quasigeostrophic balance.

Firstly, we can estimate the magnitude of the ageostrophic velocity $\left(v_{a g}\right)$ from the inviscid, quasigeostrophic $x$-momentum equation

$$
\frac{\partial u}{\partial t}+u_{g} \frac{\partial u}{\partial x}+v_{g} \frac{\partial u}{\partial y}=f v_{a g}
$$

where $u_{g}$ is the geostrophic velocity. The ageostrophic velocity approximates the divergent velocity to the extent that the ageostrophic velocity is irrotational. Assuming that the flow is steady and in thermal wind balance, $u_{g} \sim \frac{M^{2} H}{f}$. Here $M^{2}=\left|\nabla_{H} b\right|$ is the lateral buoyancy gradient in keeping with the notation for the vertical buoyancy gradient, $N^{2}=b_{z}$, where $b=-g \frac{\rho-\rho_{0}}{\rho_{0}}$. This implies

$$
v_{a g} \approx \frac{u_{g}}{f} \frac{\partial u}{\partial x}+\frac{v_{g}}{f} \frac{\partial u}{\partial y} \sim \frac{M^{2} H}{f} R o
$$

where $R o=\frac{U}{f L}$ is the Rossby number. The scaling for $w_{\text {iso }}$ is then

$$
w_{i s o} \sim v_{a g} \nabla h \sim \frac{M^{2} H}{f} R o \frac{M^{2}}{N^{2}}
$$


where $M^{2} / N^{2}$ is the slope of the isopycnals. A scaling for the total vertical velocity can be derived from the continuity equation $u_{x}+v_{y}+w_{z}=0$, as

$$
\frac{w}{H} \sim \frac{U}{L} R o
$$

which, using the scaling for the geostrophic horizontal velocity, is

$$
w \sim \frac{M^{2} H^{2}}{f L} R o
$$

The ratio is then

$$
\frac{w_{\text {iso }}}{w} \sim \frac{M^{4} H R o}{N^{2} f} \frac{f L}{M^{2} H^{2} R o}=\frac{M^{2}}{N^{2}} \frac{L}{H}
$$

For a further discussion of the physical relevance of this scaling, see Section 52.5.2. In the next two sections, we evaluate this scaling relationship and investigate the role of alongisopycnal vertical motion for nutrient fluxes using a numerical model.

\subsection{Methods}

We use the Process Study Ocean Model (PSOM) (Mahadevan et al., 1996a,c) to simulate an eddying flow field that is analyzed for the vertical transport of nutrients into the euphotic zone. The numerical model is initialized and forced with a range of horizontal buoyancy gradients to generate fronts and eddies of varying strength. This creates 3 scenarios that we use to test the scaling in equation (2.17).

The model is set up to represent an oligotrophic (nutrient-limited) region, where the mixed layer is shallower than the euphotic layer and most of the phytoplankton production occurs beneath the mixed layer. We couple a nutrient-phytoplankton model to the physical model to investigate how nutrients are supplied to sustain new production. The model forms a deep biomass maximum that is supplied nutrients by both isopycnal uplift and alongisopycnal transport. Growth within the biological model responds to a depth-attenuating light, which generates a strong nutrient gradient in the vertical, and along sloping isopycnal surfaces. 


\subsubsection{Model setup}

The numerical model is set up as a periodic channel that is $256 \mathrm{~km}$ in the east-west $(x)$ direction with closed boundaries $320 \mathrm{~km}$ apart in the north-south $(y)$ direction (Figure 22b). It extends to $1000 \mathrm{~m}$ depth and has a stretched grid in the vertical with 64 grid cells. The vertical grid spacing ranges from 3 meters at the surface to 32 meters at the bottom of the domain. We perform model runs with two horizontal grid resolutions, $1 \mathrm{~km}$ and $4 \mathrm{~km}$. The along-isopycnal diffusion of tracers is modeled using the Redi scheme (Redi, 1982) with a diffusivity of $1 \mathrm{~m}^{2} / \mathrm{s}$ in the $1 \mathrm{~km}$ resolution model and $20 \mathrm{~m}^{2} / \mathrm{s}$ in the $4 \mathrm{~km}$ resolution model. The higher resolution horizontal grid is small enough to resolve some submesoscale processes. The time step for numerical integration is 432 seconds.

The initial salinity and temperature profiles in the center of the domain are based on an Argo profile taken on December 11, 2015 in the subtropical gyre at $28.41^{\circ} \mathrm{N}, 67.16^{\circ} \mathrm{W}$ The center of the model domain is at $35^{\circ} \mathrm{N}$. The northern and southern temperature profiles are modified to create a north-south surface density gradient and two initial fronts running east-west. The initial density gradient is set with a temperature difference at the surface that decays linearly with depth to a fixed depth that varies depending on the chosen value for the surface temperature gradient. Using three different values of the north-south surface temperature variation across the $320 \mathrm{~km}$ domain: $2^{\circ} \mathrm{C}, 3^{\circ} \mathrm{C}$, and $4^{\circ} \mathrm{C}$, we achieve a range of isopycnal slopes (Figure 2-2c) that lie within observed values. All simulations initially have a 100 meter mixed layer, but isopycnal slopes differ beneath the mixed layer. The parameters used are detailed in Table 2.1.

Table 2.1: Values used for initial buoyancy gradients and isopycnal slopes

\begin{tabular}{ccccc}
\hline$\Delta T$ & $\Delta b$ & slope & Maximum depth of temperature gradient & Mixed layer depth \\
\hline $2^{\circ} \mathrm{C}$ & $0.0041 \frac{\mathrm{m}}{\mathrm{s}^{2}}$ & .0015 & $500 \mathrm{~m}$ & $100 \mathrm{~m}$ \\
$3^{\circ} \mathrm{C}$ & $0.0062 \frac{\mathrm{m}}{\mathrm{s}^{2}}$ & .001 & $600 \mathrm{~m}$ & $100 \mathrm{~m}$ \\
$4^{\circ} \mathrm{C}$ & $0.0082 \frac{\mathrm{m}}{\mathrm{s}^{2}}$ & .0005 & $600 \mathrm{~m}$ & $100 \mathrm{~m}$ \\
\hline
\end{tabular}

Vertical motion in the ocean is dominated by internal waves, which have relatively high frequencies and large, $\mathcal{O}(10 \mathrm{~m})$, vertical excursions at small spatial scales. In order to focus on quasi-balanced vertical motion of isopycnal surfaces and vertical transport along-isopycnal 

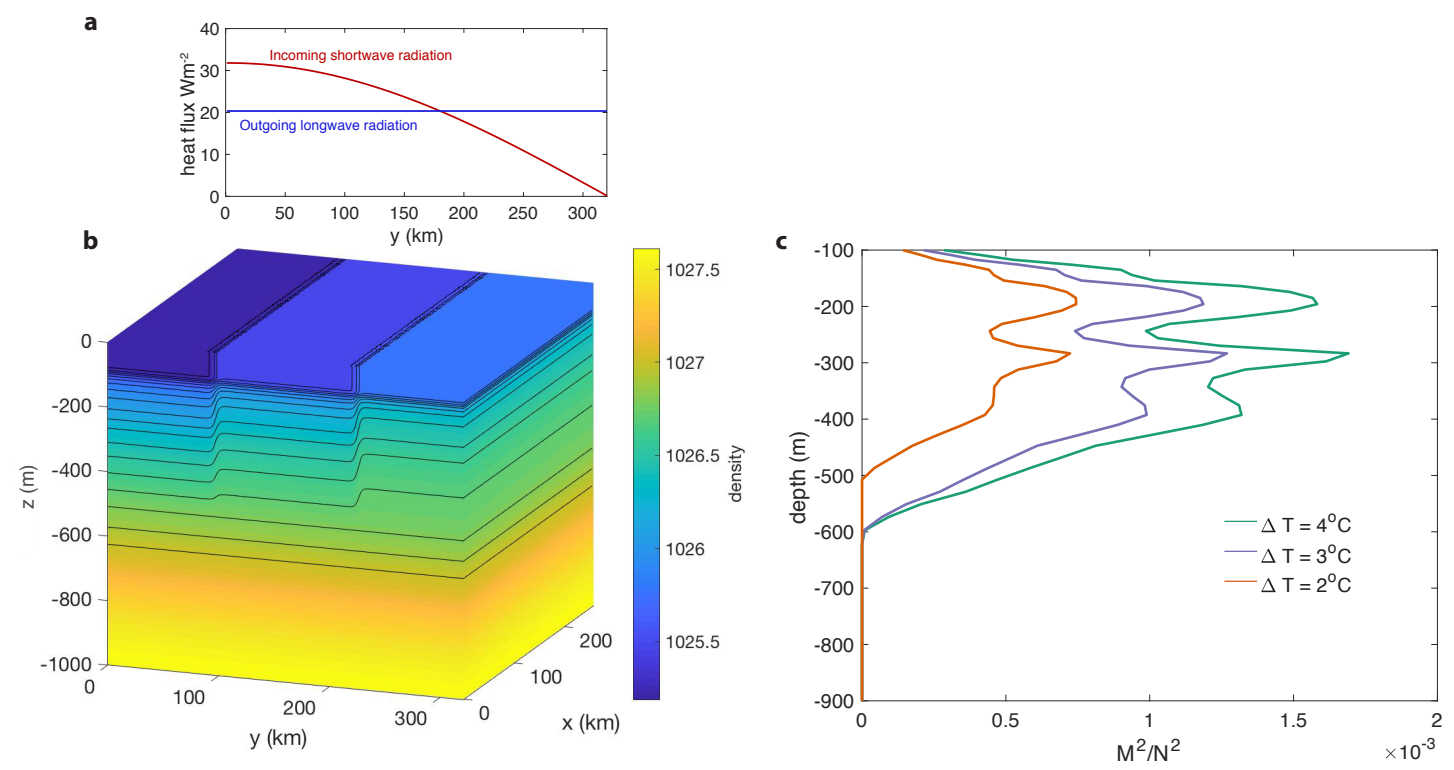

Figure 2-2: Model initialization. (a) The model is forced with a positive heat flux in the south and a negative heat flux in the north. (b) Three-dimensional view of the model domain. The model is initialized with three density profiles in order to create two fronts. (c) Initial domain-scale isopycnal slopes.

surfaces (Section 2.2), we attempt to avoid generating waves in the model by initializing the flow in geostrophic and thermal wind balance, and not using any wind forcing. Once the model is spun up, we maintain a statistically steady state with a net surface heat flux (comprising incoming shortwave - outgoing longwave radiation) that varies from a positive value in the south to negative value in the north of the domain, but integrates to zero over the whole domain with no seasonal cycle and no evaporation or precipitation (Figure 2-2a).

The model is analyzed after it has spun up for 85 days, by which time an eddy field is well-developed and the kinetic energy is in equilibrium (Figures 2-3, 2-4). The analysis period is 30 days.

\subsubsection{Decomposition of vertical velocity}

The $w_{\text {uplift }}$ and $w_{\text {iso }}$ are calculated from the model velocity field using (2.5) and (2.7). All gradients are calculated using centered differencing. Model fields are interpolated onto isopycnals and a Helmholtz decomposition of the horizontal velocity field on a density surface 
separates $\mathbf{u}=(u, v)$ into divergent $\mathbf{u}_{d i v}=-\nabla \phi$ and rotational $\mathbf{u}_{r o t}=\nabla \times \psi$ components as

$$
\mathbf{u}=-\nabla \phi+\nabla \times \psi
$$

where

$$
\nabla \cdot \mathbf{u}=-\nabla^{2} \phi
$$

Solving the Poisson equation (2.19) for the velocity potential, $\phi$, with periodic boundary conditions on the east-west boundaries and Neumann (no flux) boundary conditions on the closed north and south walls, gives the divergent velocity field $\left(u_{\text {div }}, v_{\text {div }}\right)=\left(-\phi_{x},-\phi_{y}\right)$. The vertical velocity decomposition is calculated on five isopycnal surfaces from the model output at 3.6 hour intervals so $\frac{\partial h}{\partial t} \approx \frac{\Delta h}{\Delta t}$, where $\Delta t=3.6$ hours. Over the entire analysis window, there are 200 time intervals.

\subsubsection{Biological model}

The biological model creates a deep biomass maximum due to a balance between light and nutrient availability, as is observed in many oligotrophic regions. The model is adapted from (Hodges and Rudnick, 2004) as a minimal model to create a deep biomass maximum by growth of phytoplankton $(P)$ as a function of light $(I(z))$ and nutrient $(\mathcal{N})$ uptake (modeled using a Michaelis-Menten uptake function) as

$$
\begin{array}{r}
\frac{\partial P}{\partial t}+\nabla \cdot(\mathbf{u} P)+\frac{\partial}{\partial z}\left(w_{s}(H+z) P\right)+ \\
G \frac{\mathcal{N}}{\mathcal{N}+k_{s}} I(z) P-m P^{2}=\nabla(\kappa \nabla P) \\
\frac{\partial \mathcal{N}}{\partial t}+\nabla \cdot(\mathbf{u} \mathcal{N})- \\
G \frac{\mathcal{N}}{\mathcal{N}+k_{s}} I(z) P+m P^{2}=\nabla(\kappa \nabla \mathcal{N}) \\
\text { where } I(z)=\tanh \left(e^{-k_{\text {par }} z}\right) .
\end{array}
$$

Model parameters and values are defined in Table 2.2. The sinking velocity $\left(w_{s}(H+z)\right)$ decreases linearly from $w_{s}$ at the surface $(z=0)$ to 0 at $z=H$. The quadratic phytoplankton loss term $m P^{2}$ models phytoplankton-concentration-dependent loss due to, for example, 
Table 2.2: Parameter values used in the biological model

\begin{tabular}{ccc}
\hline Parameter & Meaning & Value \\
\hline$w_{s}$ & sinking rate & $1 \mathrm{~s}^{-1}$ \\
$G$ & growth rate & $2 \mathrm{day}^{-1}$ \\
$k_{s}$ & half saturation constant & $0.05 \mu \mathrm{mol} \mathrm{kg}^{-1}$ \\
$k_{\text {par }}$ & light attenuation coefficient & $0.0461 \mathrm{~m}^{-1}$ \\
$m$ & loss rate & $0.015 \mathrm{day}^{-1}$ \\
$H$ & height of domain & $1000 \mathrm{~m}$ \\
$\kappa$ & diffusivity & $1 \mathrm{~m}^{2} / \mathrm{s}(1 \mathrm{~km} \mathrm{resolution})$ \\
& & $20 \mathrm{~m}^{2} / \mathrm{s}(4 \mathrm{~km}$ resolution $)$ \\
\hline
\end{tabular}

predation. It also increases the response to episodic nutrient inputs by reducing the phytoplankton loss rate when the phytoplankton biomass is low. The phytoplankton loss is returned to nutrients.

In this model, the euphotic depth (defined as the depth where the light level is $1 \%$ of that at the surface) is at 125 meters, using a light attenuation coefficient that is typical for subtropical gyres (Dickey et al., 2001). The euphotic depth is deeper than the mixed layer and the maximum depth of the density gradient is well below the euphotic layer for all simulations. The nutrients in the model are initialized using a linear nutrient-density relationship $\mathcal{N}=\left(\rho-\rho_{\text {mixed layer }}\right) \times 12 \mu \mathrm{mol} / \mathrm{kg}$ computed from glider time series near Bermuda (Ruth Curry, personal communication, 2016) so that nutrient concentration is initially constant on isopycnal $(\rho)$ surfaces. Nutrient concentration is nearly zero in the mixed layer. The biological model is initialized when the physical model is approaching equilibrium. The nutrient fluxes are analyzed once both the biological model and the physical model come into equilibrium, as diagnosed by small rates of domain-integrated-change of phytoplankton concentration and kinetic energy, respectively.

\subsection{Results}

\subsubsection{Effects of varying isopycnal slope}

Varying the isopycnal slope and the model grid resolution results in distinct flow fields (Figure 2-3). The dominant eddy size is smaller in the model runs with shallower isopycnal slopes than those with steeper isopycnal slopes. The high-resolution model runs have much 

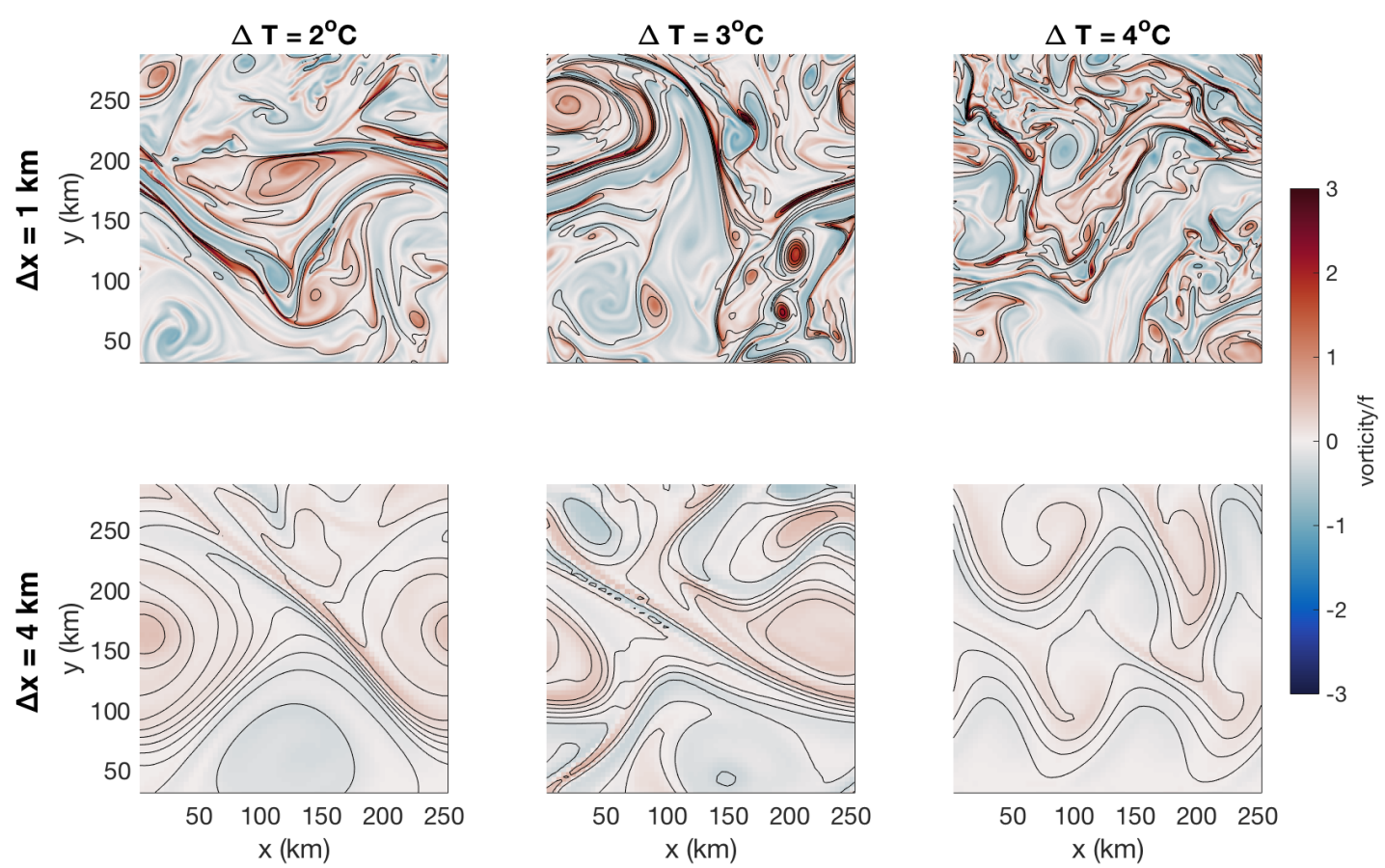

Figure 2-3: Surface relative vorticity (normalized by $f$ ) in color, overlaid with density contours (black), from the $1 \mathrm{~km}$ (Top row) and $4 \mathrm{~km}$ (Lower row) resolution models on day 10 of the analysis period. The columns, from left to right, show fields from the models with weak, medium, and steeply sloping isopycnals, generated with horizontal temperature variations of $2^{\circ} \mathrm{C}, 3^{\circ} \mathrm{C}$, and $4^{\circ} \mathrm{C}$ over the domain. Regions within $32 \mathrm{~km}$ of the north and south boundaries are eliminated from the analysis and are not shown.

larger magnitudes of surface relative vorticity, attaining values as high as $2-3 f$, compared to the low-resolution model runs, which have relative vorticity of less than $0.5 f$. The high resolution model runs have more fine-scale vorticity filaments.

All of the simulations have very similar mean nutrient distributions during the analysis period (Figure 2-4b) with deep biomass maxima just above 100 meters. The simulations with larger horizontal buoyancy gradients have slightly higher nutrient concentrations at a depth of 500 meters due to higher productivity and more sinking and remineralization at depth. By contrast, the simulations with weaker surface lateral buoyancy gradients have lower nutrients in the near-surface. Due to enhanced along-isopycnal diffusion in the low resolution model runs, there is a smaller gradient with depth in the nutrient distribution, with slightly lower nutrient concentration near 500 meter depth and slightly higher nutrient concentration in the surface. The model generates patchy and filamentous distributions of 

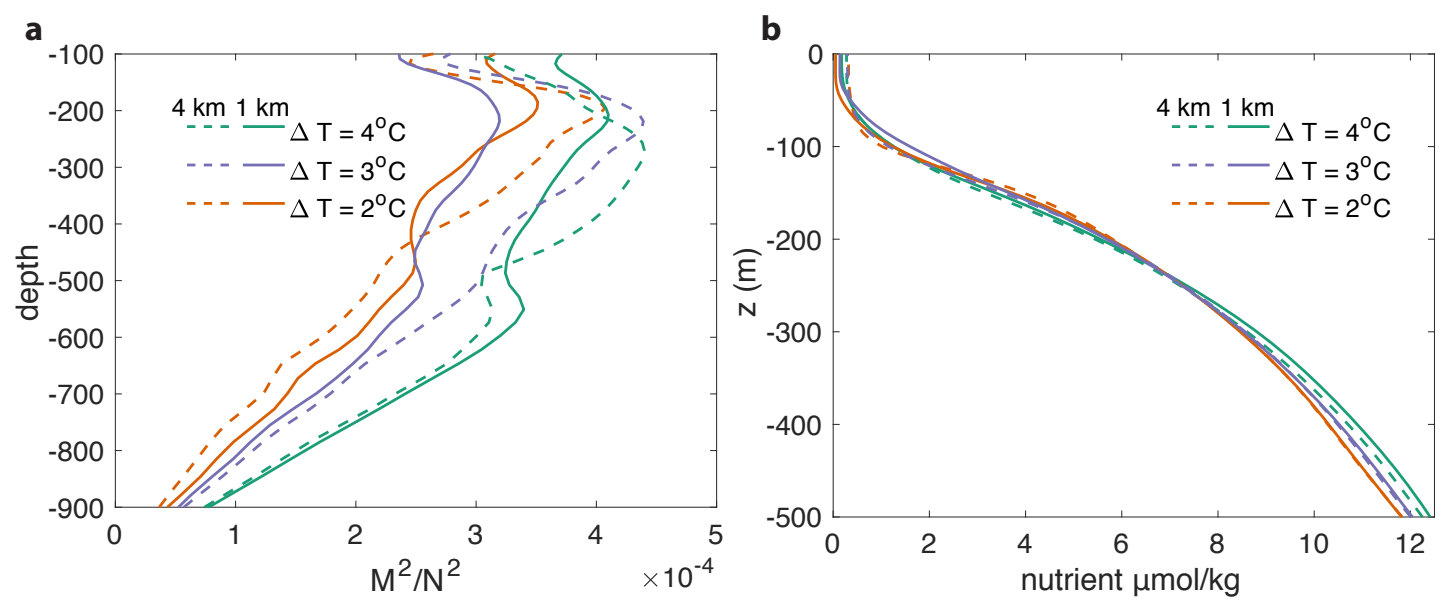

Figure 2-4: (a) Root mean squared isopycnal slopes calculated by averaging the density into $50 \mathrm{~km} \times 50 \mathrm{~km}$ bins in the horizontal during the analysis period. The upper 100 meters are not shown because the isopycnal slope is undefined in the mixed layer. (b) Mean nutrient concentration between 0 and $500 \mathrm{~m}$ during the analysis period.

nutrients and phytoplankton production (Figure 2-5c).

The high-resolution and low-resolution models that have the same initial condition maintain similar isopycnal slopes on the large scale. When the density is averaged in $50 \mathrm{~km} \times 50$ $\mathrm{km}$ boxes over the 30 day model run, the distribution of isopycnal slope with depth in the high-resolution and low-resolution models with the same initial condition is similar (Figure 2-4a). In fact, the higher resolution models have more eddy activity and so more efficiently restratify fronts through baroclinic instability, resulting in weaker isopycnal slopes at intermediate depths. However, at the local scale, the high-resolution models develop larger isopycnal slopes and density gradients (Figure 2-6).

The models with steeper isopycnal slope and higher resolution have the largest vertical velocity, as evidenced by heavier tails in the PDF of vertical velocity (Figure 2-7a). As the total vertical velocity increases, so does the proportion of the vertical motion that is along isopycnal surfaces since $w_{\text {iso }}$ also increases with the the divergent component of the horizontal velocity and the isopycnal slope in accordance with Equation (2.5).

\subsubsection{Along-isopycnal vertical velocity}

In this model configuration, within a subtropical setting, we can specify the length scale as the internal Rossby radius $L=\frac{N H}{f}$ in order to write the geometric aspect ratio $\frac{H}{L}=\frac{f}{N}$ and 

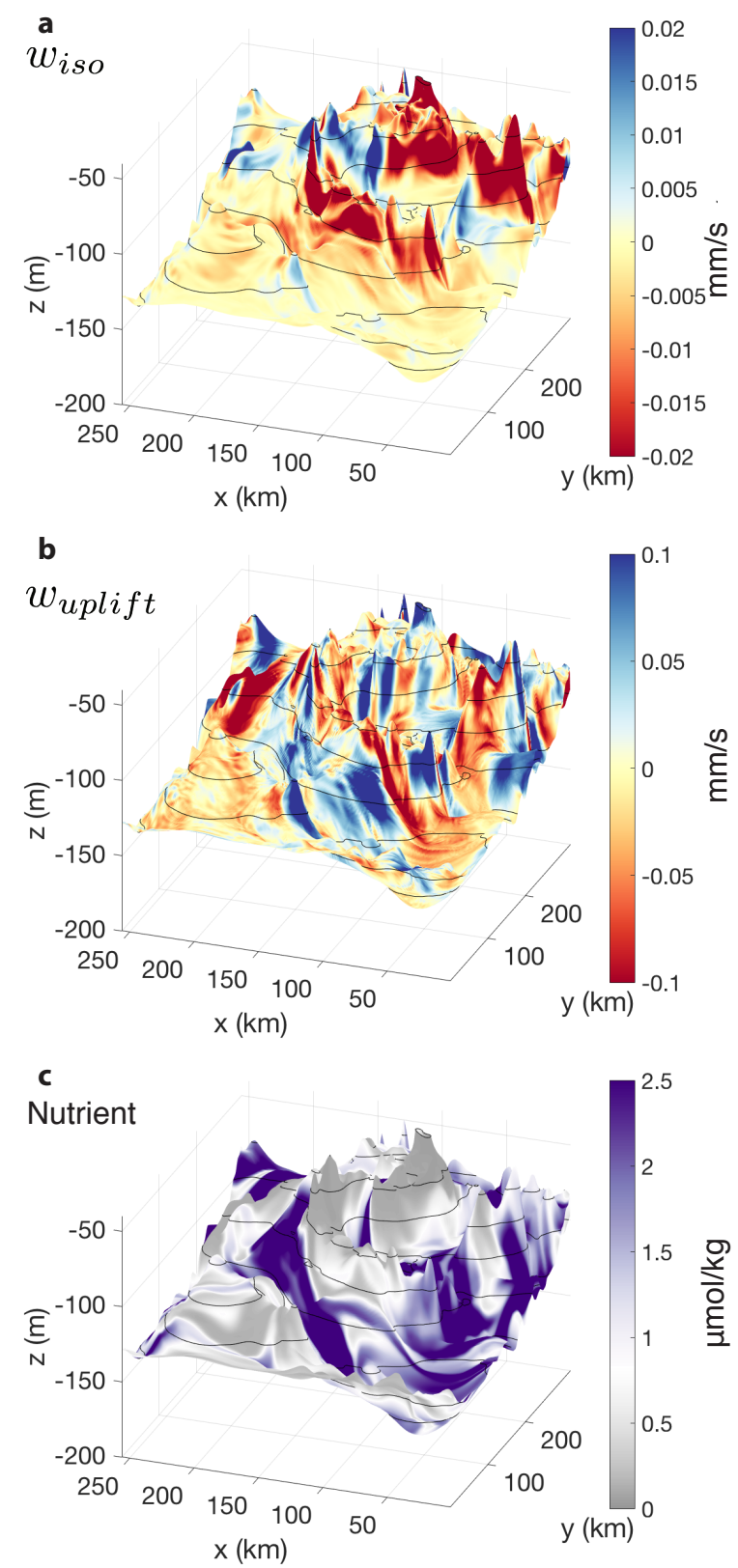

Figure 2-5: Vertical velocity and nutrient concentration at 115 days on the $\sigma=25.9$ isopycnal surface in the $1 \mathrm{~km}$ resolution model initialized with $\Delta T=2^{\circ} \mathrm{C}$. The isopycnal surface spans the depth range -150 to -50 meters; (a) $w_{\text {iso }}$ and (b) $w_{\text {uplift }}$ in $\mathrm{mm}-\mathrm{s}^{-1}$, and (c) nutrient concentration in $\mu \mathrm{mol} \mathrm{kg}-1$. The euphotic depth (1\% light level) is $125 \mathrm{~m}$.

the scaling (2.17) as

$$
\frac{w_{\text {iso }}}{w} \sim \frac{M^{2}}{N^{2}} \frac{L}{H} \sim \frac{M^{2}}{f N}
$$

The combination of changing the large-scale isopycnal slope and the model resolution, results in a range of values of this quasigeostrophic scaling parameter. In these simulations, the 


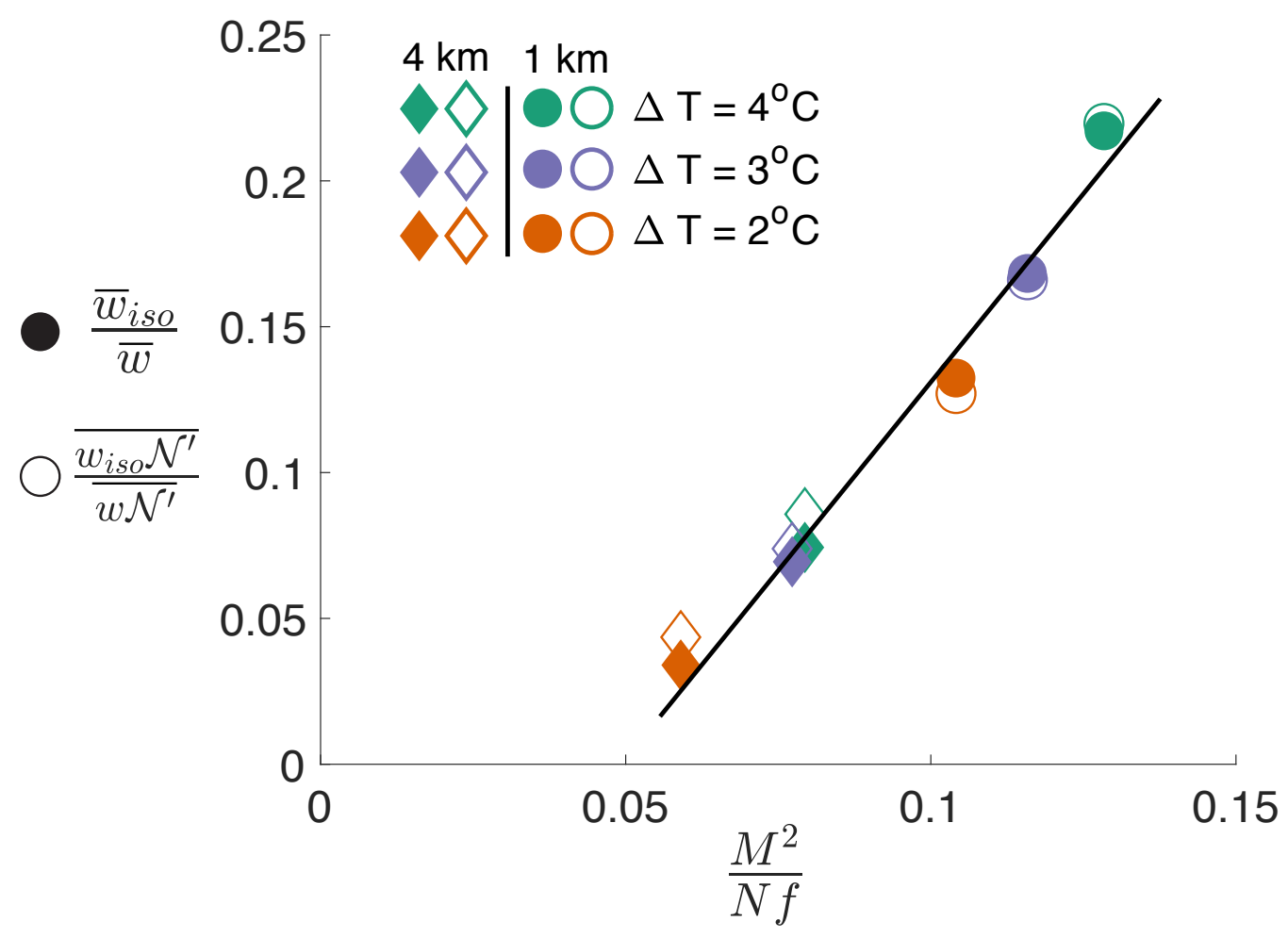

Figure 2-6: (a) Ratio of root median squared $w_{\text {iso }}$ to root median squared $w$ below the mixed layer and above 135 meters, plotted against root mean squared $\frac{M^{2}}{N f}$, evaluated between the base of the mixed layer and a depth of $135 \mathrm{~m}$ where, the aspect ratio $\frac{H}{L}=\frac{f}{N} \ll 1$. All of the averages are taken over the model domain and over the analysis period of 30 days. The slope of the best fit line is 2.58 and the intercept is -0.13 with an $R^{2}=0.98$. The diamonds are the $4 \mathrm{~km}$ resolution simulations while the circles are the $1 \mathrm{~km}$ resolution simulations. The open symbols are the ratio of the nutrient fluxes due to each of the vertical velocity components $\frac{\overline{w_{\text {iso }} \mathcal{N}^{\prime}}}{w \mathcal{N}^{\prime}}$

along-isopycnal vertical velocity $w_{\text {iso }}$ ranges from being a negligible contribution to the total vertical velocity in the low-resolution model runs, particularly with shallow isopycnal slopes, to representing about $25 \%$ of the total vertical transport in the high-resolution model run with the largest isopycnal slope (Figure 2-6). In these results, the ratio of along-isopycnal, to total, vertical velocity is domain-integrated within the pycnocline and computed on five isopycnal surfaces in the range $\sigma=25.7$ to 26.1. The linear relationship between the model-derived ratio $\frac{\overline{w_{i s o}}}{\bar{w}}$ and $\frac{M^{2}}{f N}$ (Figure 2-6, slope $=2.58, R^{2}=0.98$ ) affirms the scaling relationship (2.21).

The spatial structure of $w_{\text {iso }}$ differs from that of $w_{\text {uplift }}$ (Figure 2-8b,c and Figure 2-5a,b). 

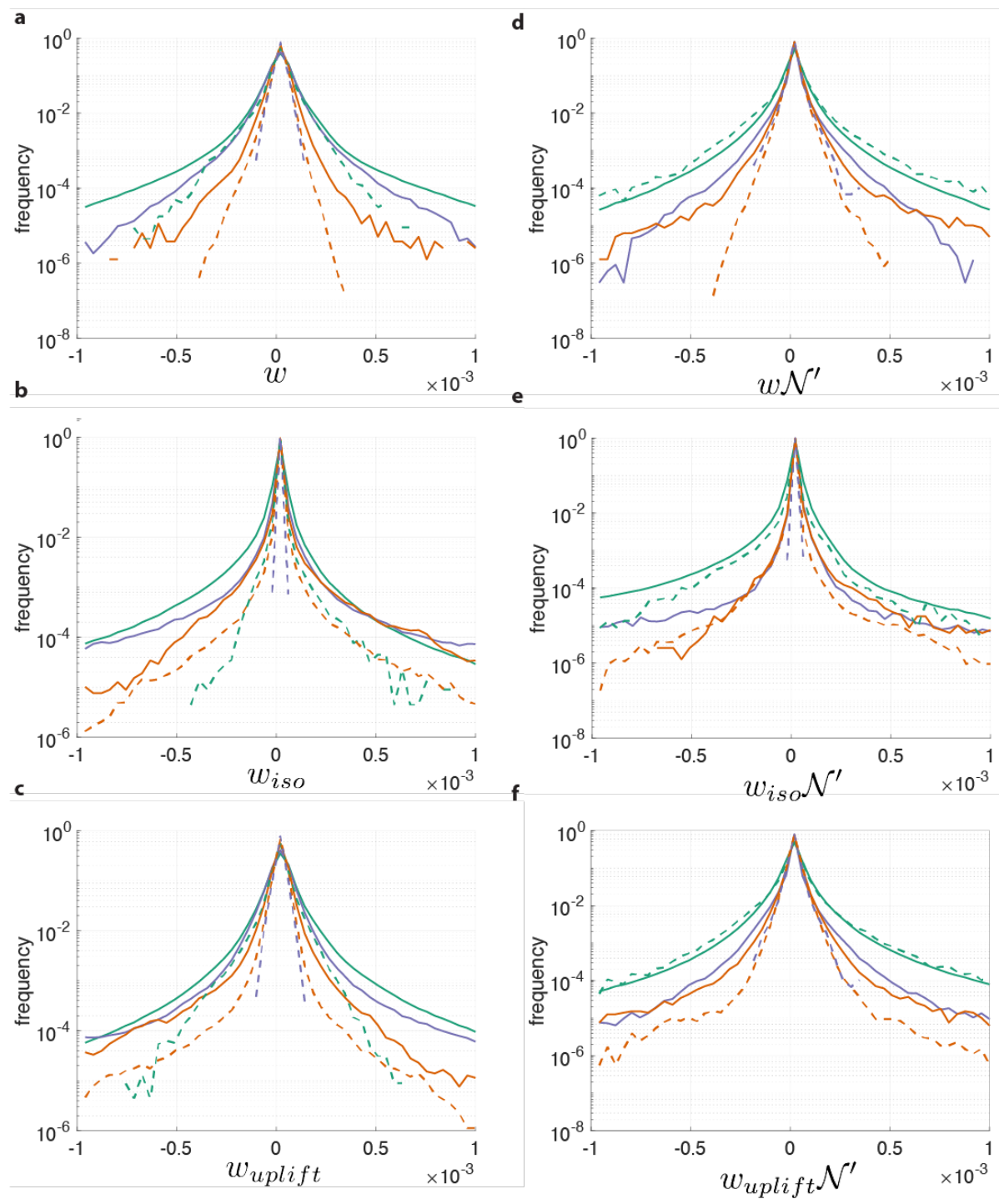

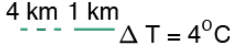

$-\cdots-\Delta T=3^{\circ} \mathrm{C}$

$---\Delta T=2^{\circ} \mathrm{C}$

Figure 2-7: Probability density functions (PDFs) on a log frequency axis: (a-c) Vertical velocity components near the euphotic depth (100-135 meters), and (d-f) vertical nutrient flux components at the same depth. (a) Vertical velocity $(w)$, (b) along-isopycnal vertical velocity $\left(w_{\text {iso }}\right),(\mathrm{c})$ isopycnal uplift velocity $\left(w_{\text {uplift }}\right),(\mathrm{d})$ nutrient flux due to total vertical velocity $\left(w \mathcal{N}^{\prime}\right)$, (e) nutrient flux due to along-isopycnal vertical velocity $\left(w_{i s o} \mathcal{N}^{\prime}\right)$, (f) nutrient flux due to isopycnal uplift vertical velocity $\left(w_{\text {uplift }} \mathcal{N}^{\prime}\right)$. In each case, PDFs can be compared for the $4 \mathrm{~km}$ and $1 \mathrm{~km}$ resolution models and the different isopycnal slopes $\left(\Delta \mathrm{T}=2^{\circ}, 3^{\circ}, 4^{\circ} \mathrm{C}\right)$.

The along-isopycnal vertical velocity, $w_{i s o}$, has a shallower wavenumber spectrum and more power at small scales (Figure 2-8b,c). With a weaker isopycnal slope $\left(\Delta \mathrm{T}=2^{\circ} \mathrm{C}\right.$ and $\Delta \mathrm{T}$ 


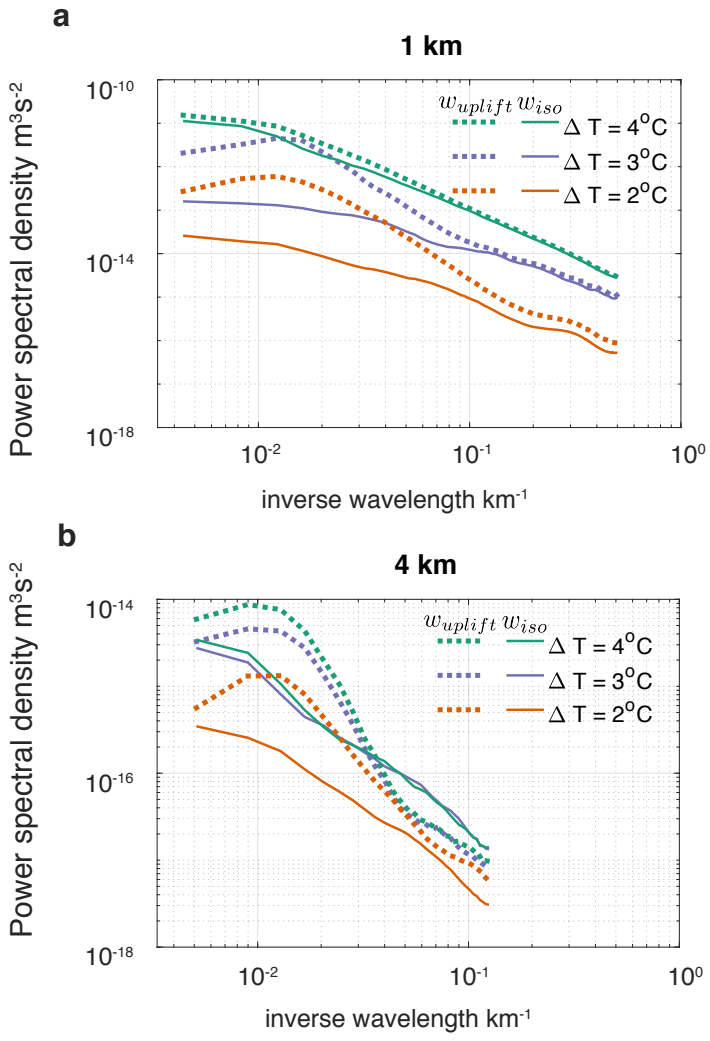

Figure 2-8: Spectrum of $w_{\text {uplift }}$ and $w_{\text {iso }}$ on the $\sigma=25.9$ isopycnal surface for each of the various isopycnal slopes $\left(M^{2} / N^{2}\right)$ modeled using a horizontal temperature difference $\Delta \mathrm{T}=2^{\circ}, 3^{\circ}$ and $4^{\circ} \mathrm{C}$. (a) $1 \mathrm{~km}$ resolution models (b) $4 \mathrm{~km}$ resolution models.

$=3^{\circ} \mathrm{C}$ ) the relationship between the $w_{\text {iso }}$ spectrum and the $w_{\text {uplift }}$ spectrum is similar in the low-resolution and high-resolution simulations. With the larger buoyancy gradient, $w_{\text {iso }}$ has relatively more power across all wavenumbers in the high-resolution simulation than in the low-resolution simulation. With the largest buoyancy gradients $\left(\Delta \mathrm{T}=4^{\circ} \mathrm{C}, 1 \mathrm{~km}\right.$ resolution), $w_{\text {iso }}$ has more power at large scales than in the other simulations and the spectra of $w_{\text {iso }}$ and $w_{\text {uplift }}$ are nearly identical.

At the surface, the vertical velocity is skewed such that the negative vertical velocities (downward) are more intense than the positive vertical velocities (not shown). Deeper in the water column, in the depth range 100-135 meters, which is near the euphotic depth, the distribution of the total vertical velocity is symmetric (Figure 2-7a). However, the distribution of along-isopycnal vertical velocity is skewed even near the euphotic depth, with more intense downward vertical velocities (Figure 2-7b). The skewness in $w_{\text {iso }}$ is more 
intense in the high resolution and steep isopycnal slope simulations. Relatedly, $w_{\text {uplift }}$ is slightly positively skewed (Figure 2-7c).

\subsubsection{Nutrient fluxes}

The vertical flux of the nutrient-like tracer depends not just on the magnitude of the vertical motion, but also on the covariance between the tracer anomaly and the vertical velocity $\left(\overline{w \mathcal{N}^{\prime}}\right)$. We define the nutrient anomaly as the anomaly from the mean concentration on a given isopycnal surface $\left(\mathcal{N}^{\prime}=\mathcal{N}-\overline{\mathcal{N}_{\text {iso }}}\right)$ in order to focus on flux along isopycnal surfaces, and because nutrient concentration is initially correlated with density. In these simulations, the magnitude of the vertical nutrient flux induced by along-isopycnal vertical transport increases in proportion to vertical along-isopycnal motion such that $\frac{\overline{w_{\text {iso }} \mathcal{N}^{\prime}}}{w \mathcal{N}^{\prime}} \approx \frac{\overline{w_{\text {iso }}}}{\bar{w}}$, where the overbar indicates a spatial average over the domain. This is due to low coherence (calculated, but not shown) between all components of the vertical velocity $\left(w, w_{i s o}\right.$, and $\left.w_{\text {uplift }}\right)$ and the nutrient anomaly in this model, also evident from the large depth range of the nutricline (Figure 2-5c). Here, the total nutrient flux depends on both model resolution and isopycnal slope (Figure 2-7d-f). Due to the low covariance between vertical velocity and nutrient anomaly, the negative skewness in the distribution of $w_{i s o}$ necessarily results in a negatively skewed vertical nutrient flux due to along-isopycnal vertical velocity (Figure $2-7 \mathrm{e})$.

\subsection{Discussion}

\subsubsection{Global context}

We assess the extent to which the vertical flux (of a tracer) occurs along sloping isopycnal surfaces in the pycnocline for different regions of the world's oceans by computing the scaling factor $\frac{M^{2} L}{N^{2} H}$ in a high resolution global MITgcm run with $1 / 48^{\circ}$ resolution in the horizontal and 90 vertical levels (Su et al., 2018). The horizontal resolution of the MITgcm is approximately $2 \mathrm{~km}$ at mid latitudes, which is midway between the two model resolutions used in the smaller domains analyzed in this study. While the necessity of performing a Helmholtz decomposition to calculate $w_{\text {iso }}$ makes computation of the ratio $\frac{w_{\text {iso }}}{w}$ computa- 


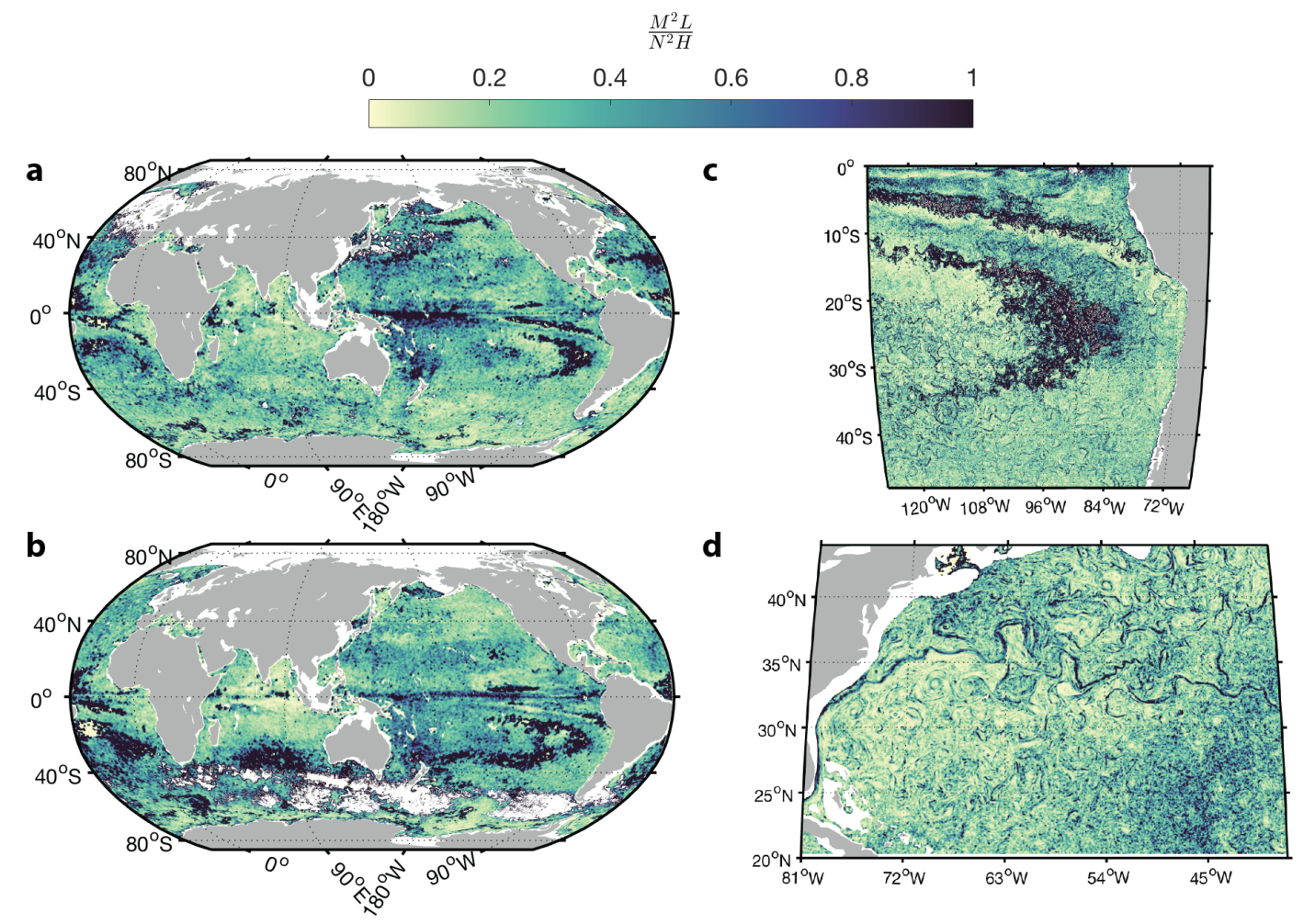

Figure 2-9: Scaling factor $\frac{M^{2} L}{N^{2} H}$ is calculated at $150 \mathrm{~m}$ depth from global, $1 / 48^{\circ}$-resolution MITgcm model fields on (a, c) January 15, 2012 and (b, d) June 15, 2012. Areas where the mixed layer is deeper than 150 meters or where the total water column depth is less than 150 meters are shaded white. The inverse aspect ratio of the velocity is calculated as $\frac{L}{H}=\sqrt{\frac{\partial u^{2}}{\partial z}+\frac{\partial v^{2}}{\partial z}} / \sqrt{\frac{\partial u^{2}}{\partial y}+\frac{\partial v^{2}}{\partial x}}$. The computed ratio is smoothed using a Gaussian filter with a $120 \mathrm{~km}$ window in figures (a) and (b) and a $12 \mathrm{~km}$ window in figures (c) and (d). Simulations provided by Christopher N. Hill.

tionally costly on such a large domain, the scaling factor $\frac{M^{2} L}{N^{2} H}$ can be easily computed from the global model fields (Figure 2-9). For the global model, it is inappropriate to choose the length scale $L=\frac{N H}{f}$, which is based on the mid-latitude baroclinic instability. Though this simplifies the scaling factor to $\frac{M^{2}}{N f}$ as in Figure 2-6, the simplification is applicable only in the subtropical gyres, and not near the equator and poles because of the inverse dependence on the Coriolis frequency. The inverse aspect ratio of the flow is instead calculated globally using the magnitudes of the velocity gradients as $\frac{L}{H}=\left(u_{z}^{2}+v_{z}^{2}\right)^{1 / 2}\left(u_{y}^{2}+v_{x}^{2}\right)^{-1 / 2}$.

The extent to which the vertical motion is along sloping isopycnal surfaces varies geographically and depends on the flow regime. The scaling factor $\frac{M^{2} L}{N^{2} H}$ is large in the low 
latitudes, where the aspect ratio $\left(\frac{H}{L}\right)$ of the velocities becomes small. In the mid latitudes, $\frac{M^{2} L}{N^{2} H}$ becomes relatively large, reaching values of $40-50 \%$ in the highly energetic regions such as the Gulf Stream (Figure 2-9d) and Kuroshio, as well as the California Current and Hum-

boldt current (Figure 2-9c). In the high-latitudes, the large values of $\frac{M^{2} L}{N^{2} H}$ are mostly due to large isopycnal slopes. There is some seasonality to the scaling factor, which is larger during the winter, particularly in high-latitudes.

This suggests that vertical motion along sloping isopycnal surfaces is particularly important in low latitudes and in highly energetic regions, or fronts, with large isopycnal slope. Though the seasonality in the scaling suggests a seasonality in the importance of along-isopycnal vertical flux, its importance for production may be diminished in winter, because nutrient flux during the wintertime is less important for productivity than in the summertime. The scaling suggests that the vertical flux along isopycnal surfaces could be the dominant vertical flux pathway in some regions.

\subsubsection{Along-isopycnal transport}

Using this decomposition, we find that $w_{\text {iso }}$ and $w_{\text {uplift }}$ are anti-correlated over much, but not all, of the domain. Although different process drive $w_{\text {iso }}$ and $w_{\text {uplift }}$, it should be pointed out that they are not mutually independent, and they co-occur in some dynamical situations. For example, as the stratification changes due to an uplift process, water must move along a sloping isopycnal surface. On the other hand, $w_{\text {iso }}$ can occur independently of $w_{\text {uplift }}$ along a steady isopycnal surface, and a linear wave is an example of a process where only $w_{\text {uplift }}$ is important. Though $w_{\text {iso }}$ and $w_{\text {uplift }}$ co-occur in our model eddy field, the difference in the distribution of their spatial scales suggests process-level differences in their contribution to vertical motion.

Much of the subtropical gyres are nutrient-limited and it has been proposed that the eddy-driven supply of nutrients is particularly important in these regions (McGillicuddy et al., 1998b; Ascani et al., 2013). In the case of mid-latitude baroclinic instability, using the thermal wind relation $U_{z}=\frac{M^{2}}{f}$ and $\frac{L}{H}=\frac{N}{f}$, enables us to express (2.17) in terms of 
$R i_{b}$, the thermally balanced Richardson number as

$$
\frac{w_{\text {iso }}}{w} \sim \frac{M^{2}}{N f}=\left(\frac{U_{z}^{2}}{N^{2}}\right)^{-1 / 2}=R i_{b}^{-1 / 2} .
$$

With steeper isopycnal slope, the total vertical velocity increases, but so does the proportion of the vertical velocity that is due to motion along sloping isopycnal surfaces. In our model runs, the largest value of $\frac{M^{2}}{N f} \approx 0.13$, equivalent to $R i_{b}=59$. This raises questions about the appropriateness of this quasigeostrophic scaling at smaller Richardson number and in higher resolution models, though it may hold beyond the quasigeostrophic regime. In the semigeostrophic solution of the ageostrophic overturning circulation, which is more appropriate for small Richardson number, the flow is more aligned with isopycnal surfaces than in the quasigeostrophic regime (Hoskins, 1982). This suggests that more of the vertical flux is along-isopycnal, rather than due to the motion of the isopycnal surfaces. If we assume that the equilibrated state of mixed layer restratification by inviscid geostrophic adjustment is

$N^{2}=\frac{M^{4}}{f^{2}}$ (Tandon and Garrett, 1994), we find that $\frac{w_{i s o}}{w} \rightarrow 1$, even as as $R i_{b} \rightarrow 1$. However, the scaling relationship does not account for processes like mixed layer instability, which can restratify a weakly stratified mixed layer more efficiently than geostrophic adjustment (Fox-Kemper et al., 2008). Other processes, such as near-inertial oscillations (Tandon and Garrett, 1995), wind-induced Ekman transport (Whitt et al., 2017) and symmetric instability (Thomas et al., 2013), may also contribute to the along-isopycnal nutrient fluxes, but are not considered here. Moreover, mixed layer processes could be important for vertical tracer flux even beneath the mixed layer through the coupling of submesoscale and mesoscale processes (Ramachandran et al., 2014).

\subsubsection{Spatial scales}

Previous studies (Mahadevan and Archer, 2000; Lévy et al., 2001; Rosso et al., 2014) have shown that the magnitude of the vertical velocity and vertical nutrient flux increases with increasing model resolution. Our results are consistent, but additionally reveal that $w_{\text {iso }}$ is more poorly represented in low-resolution model simulations than $w_{\text {uplift }}$. Since $w_{\text {iso }}$ has a relatively flat wavenumber spectrum compared to that of $w_{\text {uplift }}$, representation of 
small scales in models is particularly important for resolving the contribution to vertical velocity from $w_{i s o}$. The high-resolution model runs generate larger density gradients at the local scale, resulting in a higher proportion of the vertical motion being due to $w_{\text {iso }}$. In addition, in the simulation with the largest horizontal buoyancy gradient, representation of the smallest spatial scales results in more power of $w_{\text {iso }}$ across spatial scales.

\subsubsection{Nutrient fluxes}

These results suggest a path forward for parameterizing subgrid scale tracer flux in mesoscale eddy resolving and eddy permitting models that is distinct from the parameterization of buoyancy fluxes. The Redi scheme (Redi, 1982), which is used in our model runs, parametrizes horizontal tracer mixing as diffusion acting along isopycnal surfaces, with the vertical diffusion of tracer proportional to the isopycnal slope squared (Gnanadesikan et al., 2015). We find that in addition to the isopycnal slope, the velocity gradients are also important for determining the vertical tracer flux, although these may be less important for horizontal tracer fluxes. Parameterizing the along-isopycnal tracer flux is important for appropriately representing the isopycnal uplift flux as well, because both components of the vertical motion contribute to the nutrient anomalies.

In these model simulations, the coherence between vertical velocity and nutrient anomaly was relatively low. The nutrient anomalies may persist for longer times than the vertical velocity anomalies, which have high-frequency fluctuations (Pasquero, 2005). For this reason, the coherence between vertical velocity and nutrient anomalies may depend on the nutrient uptake timescales, with faster uptake resulting in a higher coherence between the vertical velocity and nutrient anomaly. The coherence between the vertical velocity and the nutrient anomaly is very noisy in the high-resolution simulation, due to a very filamentous nutrient distribution along isopycnal surfaces that is not necessarily in phase with the vertical velocity. In the case of a tracer like nutrient, an increased rate of uptake will enhance the vertical gradient of the tracer and lead to more coherence between the vertical velocity and nutrient anomalies.

Along-isopycnal transport of nutrients could be particularly important for supplying deep biomass maxima, which are common in oligotrophic regimes and tend to occur around 
100 meters depth (Cullen, 2015). The depth of the mixed layer relative to the depth of light availability and the nutrient content of the mixed layer are known to be important influences on phytoplankton growth (Sverdrup, 1953). Since production can be enhanced by along-isopycnal transport, the depth of lateral density gradients relative to the depth of the nutricline may be another important factor for total productivity.

In these simulations, particularly those with the largest density gradients, the nutrient flux is negatively skewed due to the negatively skewed vertical velocity field combined with a low coherence between nutrient anomaly and vertical velocity. Some previous studies have found that increasing grid resolution in highly energetic regions can decrease phytoplankton productivity (Gruber et al., 2011; Lathuiliere et al., 2010) due to an export of phytoplankton or unutilized nutrients. This is likely due to $w_{i s o}$ contributing to a negatively skewed flux and the along-isopycnal export of nutrients before the nutrient can be consumed.

As the measurement of vertical velocity in the ocean becomes possible through Lagrangian floats (D'Asaro et al., 2018) or direct measurement (Thurnherr et al., 2015), this framework could be applied to observational data to separate vertical transport due to linear waves and wave-like vertical motion by eddies, from tracer transport along sloping isopycnal surfaces. Observations by profiling floats have been able to observe motion of isopycnal surfaces and motion of nutrient isosurfaces (Ascani et al., 2013). While these may be correlated, the link is not necessarily causal. Instead, the vertical flux of tracers along sloping isopycnal surfaces should be understood as potentially distinct from the vertical flux of buoyancy.

\subsection{Conclusions}

The decomposition of the subsurface vertical velocity, $w$, into an along-isopycnal component $w_{\text {iso }}$, and an isopycnal uplift component $w_{\text {uplift }}$, enables us to mechanistically understand vertical motion and derive a scaling for the ratio $\frac{w_{i s o}}{w} \sim \frac{M^{2} L}{N^{2} H}$. Numerical simulations performed with $1 \mathrm{~km}$ and $4 \mathrm{~km}$ grid resolutions satisfy the scaling relationship for a range of isopycnal slopes. The scaling suggests a new parameterization for the vertical component of tracer transport along isopycnal surfaces in eddy-permitting and eddy-resolving models. $w_{\text {iso }}$ accounts for $5-25 \%$ of the vertical transport of nutrients in simulations of the mid-latitude 
eddy field, but is potentially the dominant mechanism for vertical transport in regions with sleeply sloping isopycnals. While steeper isopycnal slopes and higher model resolution results in larger, and more negatively skewed vertical velocity, $w$, we find this is mostly due to the increased magnitude and negative skewness in $w_{\text {iso }}$ compared to $w_{\text {uplift }}$. Vertical motion along sloping isopycnal surfaces is particularly important at small scales, in regions with large lateral density gradients, and at low latitudes. While $w_{\text {iso }}$ does not contribute a buoyancy flux, it is important for the vertical flux of biogeochemical tracers with strong vertical gradients, and for the supply of nutrients to phytoplankton in oligotrophic regions. 


\title{
Chapter 3
}

\section{Biophysical feedbacks of}

\section{phytoplankton growth on nutrient}

\section{supply and diversity}

\begin{abstract}
The rate at which nutrients are supplied to phytoplankton in the sunlit surface layer of the ocean depends not only on physical transport processes, but also on the growth rate of phytoplankton. Vertical transport of nutrient, which depends on the time-integrated covariance between vertical velocity and the nutrient's anomaly from its mean concentration has a nonlinear dependence on the nutrient uptake rate. A subtropical oceanic eddy field has a wide range of vertical velocities and frequencies with which water moves (up and down) within the nutricline. Nutrient flux is maximized when the decorrelation timescale of the vertical velocity matches the uptake rate of the nutrients. If each water parcel supplies nutrient to maximize the rate of new production, we find that the average phytoplankton growth rate is $1 / 3$ day $^{-1}$. The associated time scale (3 days) is that of sub-inertial submesoscale dynamics. The spatial structuring in the frequency of vertical motion experienced by water parcels in different physical features of the flow suggests a growth-transport feedback mechanism for generating diversity in phytoplankton community structure.
\end{abstract}

\subsection{Introduction}

Phytoplankton account for about half the photosynthetic production of organic matter and oxygen on the planet. Their production relies on sunlight and nutrients, like nitrate, phosphate, silica and minute amounts of iron, zinc and cobalt. The diverse community of phy- 
toplankton and the heterotrophic ecosystem that it supplies affects the depth and efficacy of primary production, as well as the cycling of carbon and other elements. Most of the world's ocean is oligotrophic (depleted of nutrients) in the sunlit (euphotic) layer where they are taken up by phytoplankton, but nutrient concentration increases with depth below the euphotic layer. In such regions, the production of phytoplankton relies on the physical transport of nutrient-enriched water from depth to the euphotic zone where light enables photosynthesis (McGillicuddy et al., 1998a). Over long spatial and temporal scales, the system is balanced such that the rate of export of organic matter is determined by the rate of nutrient input that contributes to photosynthetic carbon fixation (Falkowski et al., 1998; Ducklow et al., 2001).

The upward transport of nutrient-rich water occurs via a range of mechanisms, including surface boundary layer turbulence, wind-driven upwelling, coastal upwelling, eddy uplift and frontal instabilities (Denman and Gargett, 1983; Lipschultz et al., 2002). In the pelagic ocean, the physical supply of nutrients induced by vertical advection associated with fronts and eddies (Jenkins and Goldman, 1985) is thought to limit the rate of new production, which is the rate of phytoplankton production fueled by a fresh supply of macronutrients from outside the euphotic layer. Vertical velocities are typically $10^{-3}$ to $10^{-4}$ times smaller than the horizontal velocities associated with ocean currents and eddies on scales of 1$100 \mathrm{~km}$. But, submesoscale dynamics, associated with strong vertical vorticity (of the order of the planetary vorticity $f$ ) can result in vertical velocities of $\mathcal{O}(100) \mathrm{m} /$ day on spatial scales $\mathcal{O}(1 \mathrm{~km})$. These rapid vertical motions are thought to be particularly influential for phytoplankton growth.

Besides physical processes, the gradient in the mean nutrient distribution and the anomalies in concentration from the mean distribution also affect nutrient transport. Conversely, physical transport, as well as biological and chemical processes that create sinks (or sources) for nutrients, affect their distribution. The spatial heterogeneity of reactive tracers and biological fields in the ocean is dependent on the Damköhler number, $D a$, which is the ratio of the advection to reaction time scales (López et al., 2001; Abraham, 1998; Mahadevan and Archer, 2000). It controls the nonlinear relationship between reaction products and physical decorrelation timescales. Da affects the efficiency of transport (Mahadevan and Campbell, 
2002; Pasquero et al., 2005; Smith et al., 2016), which is optimized for $D a=\mathcal{O}(1)$.

Hence, the nutrient supply rate cannot be assessed from physics alone; it also depends on the rate of nutrient uptake by phytoplankton, which is closely related to the growth rate, a physiological characteristic that depends on the kind of phytoplankton, its size, the ambient temperature, light and nutrient availability. Ocean ecosystems are highly heterogeneous with phytoplankton cell sizes and growth rates varying by orders of magnitude (Laws, 2013). This diverse range of phytoplankton has a wide range of growth rates that are both affected by and affect the nutrient supply rate.

\subsection{Nutrient supply and uptake}

Due to the dependence of phytoplankton growth on light, and the ocean's density stratification and Earth's rotation, which inhibit vertical movement of water, oceanic biogeochemical tracers typically have a strong depth dependence, with relatively weak lateral variability. In the oligotrophic, subtropical oceans, the area- and time-averaged mean vertical profile of nutrient $N_{0}(z)$ is depleted in the near-surface euphotic layer (upper $100 \mathrm{~m}$ ), and has a strong vertical gradient (nutricline) in the region of strong density stratification (pycnocline) approximately between 100-500 m (Supp. Fig. C-2). The nutrient concentration is altered by advection and nutrient uptake (or resupply), which we model as a linearized resourcelimited source/sink function $f(N)=-\lambda\left(N-N_{0}(z)\right)$ (Abraham, 1998; López et al., 2001). This function is a linearization of logistic growth or decay near carrying capacity (Supp. Text A.1). Our simple model for nutrient

$$
\frac{\partial N}{\partial t}+\nabla \cdot(\mathbf{u} N)=-\lambda\left(N-N_{0}(z)\right)
$$

accounts for transport by the ocean velocity field $\mathbf{u}=(u, v, w)$ and the uptake (resupply) of nutrients (Mahadevan and Archer, 2000) and averages over several aspects of the ecosystem and the interactions of its components due to grazing, mortality, detritus production, and bacterial remineralization. Here, $\lambda$ is the rate of uptake or resupply (per unit time). For practical purposes, we take $\lambda$ to be depth-independent in our model and encode all the depth 
dependence in $N_{0}(z)$, but in a general model $\lambda$ could vary in space and time, or be a function of temperature. By defining the nutrient anomaly $N^{\prime}(x, y, z, t) \equiv N(x, y, z, t)-N_{0}(z)$ and taking the horizontal average, denoted by \langle\rangle of (3.1), over a region with no large-scale horizontal gradients in $N$, we are left with a balance between vertical transport and nutrient uptake or resupply, because the spatial average of the vertical velocity $(w)$ vanishes, $\langle w\rangle=0$ and $\partial_{x}\left\langle u N^{\prime}\right\rangle=\partial_{y}\left\langle v N^{\prime}\right\rangle=0$, such that

$$
\left\langle\partial_{t} N^{\prime}\right\rangle+\left\langle\partial_{z}\left(w N^{\prime}\right)\right\rangle=-\left\langle\lambda N^{\prime}\right\rangle
$$

Nutrient anomalies are generated by the vertical advection of nutrient and restored to the equilibrium profile at a rate $\lambda$. Where $N^{\prime}>0$, nutrient is consumed, as by the new production of phytoplankton. Where $N^{\prime}<0$, nutrient is restored to its equilibrium profile, and the resupply represents remineralization. Time-averaged over a the long term (several months) we expect a steady state in which the nutrient flux at the base of the euphotic layer $\left(z=-z_{e}\right)$ is balanced by the nutrient consumption above it $\left\langle w N^{\prime}\right\rangle_{z_{e}}=-\int_{-z_{e}}^{0}\left\langle\lambda N^{\prime}\right\rangle d z$. In a time- and area-averaged sense, the net uptake (resupply) is equivalent to the net community production $(\mathrm{NCP})$, which over the long term, is balanced by the net export and is often, as it is here, conceptualized in terms of first order rate kinetics (Woodwell and Whittaker, 1968; Emerson, 2014).

\subsubsection{Dependence of flux on the frequency of vertical velocity and uptake rate}

The dependence of the nutrient flux on the covariance between $w$ and $N^{\prime}$ can be demonstrated conceptually (Fig. 3-1) by using an oscillating vertical velocity with a characteristic timescale $\tau w=w_{0} \sin \left(\frac{2 \pi \tau^{-1} t}{)}\right.$. When $\lambda \gg \tau^{-1}$ (fast uptake), the nutrient anomaly is in phase with the vertical velocity, but its magnitude is small because nutrients are consumed immediately (Fig. 3-1B). Consequently, the magnitude of the flux is small. By contrast,

when $\lambda \ll \tau^{-1}$ (uptake is slow), nutrients remain unconsumed and the nutrient anomaly is large, but out of phase with the vertical velocity (Fig. 3-1D). Because the nutrient anomaly and vertical velocity are out of phase, there are times of positive and negative flux, which, 


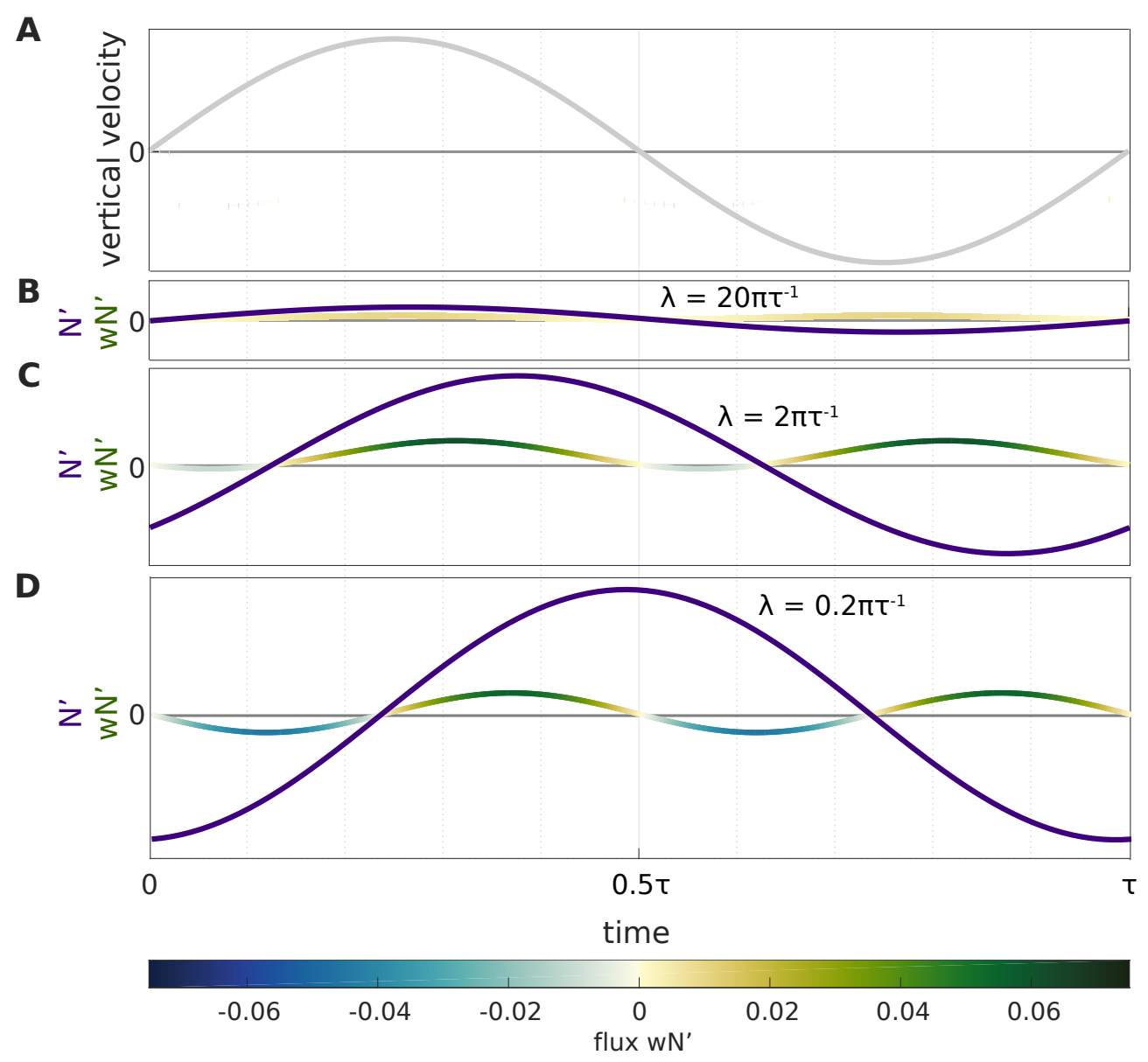

Figure 3-1: Schematic showing the relationship between vertical velocity, nutrient anomaly and nutrient flux.

when averaged in time (and denoted by an overbar), results in a small net vertical flux $\overline{w N^{\prime}}$. The net nutrient flux is maximized between those two extremes, when $\lambda=2 \pi \tau^{-1}$ for a sinusoidal vertical velocity with angular frequency $2 \pi \tau^{-1}$, such that nutrient anomalies have a larger magnitude relative to the fast growth case and a $45^{\circ}$ phase shift relative to the vertical velocity (Fig. 3-1C) so that there is a net positive flux when averaged over a time period (Supp. Text A.3).

\subsubsection{Nutrient fluxes in an oligotrophic eddy field}

In ocean observations, vertical motions occurring on a wide range of spatial and temporal scales contribute to biological tracer fluxes, resulting in more complex patterns of eddy correlation than in a simple oscillatory flow field. We use a numerical ocean model to 

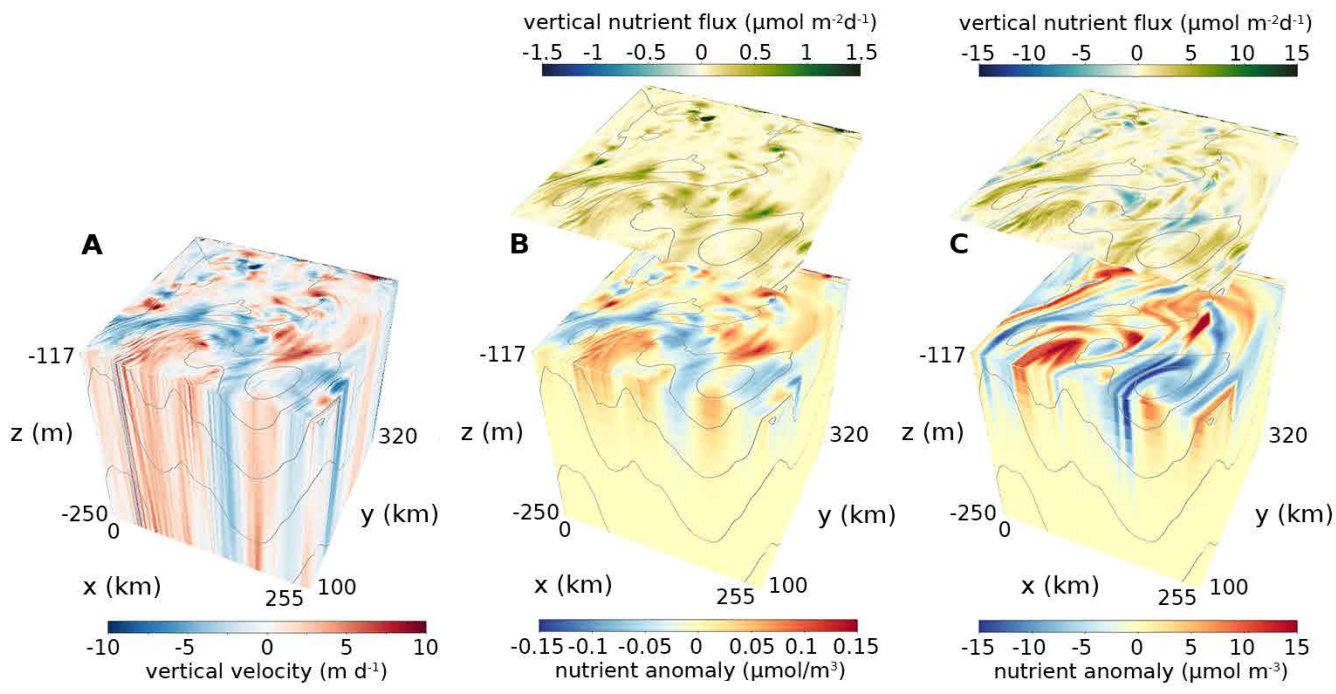

Figure 3-2: Snapshots of vertical velocity, nutrient anomaly, and vertical nutrient fluxes on model day 135 (A) Vertical velocity at $z=-117 \mathrm{~m}$. (B and C) Lower cube is nutrient anomaly, the upper panel is the vertical nutrient flux at $z=-117 \mathrm{~m}$ with uptake rates (B) $\lambda=0.015$ day $^{-1}$ and (C) $\lambda=1.5$ day $^{-1}$

examine the dependence of the vertical nutrient flux on the uptake rate $\lambda$ in the fully developed, nonlinear oceanic regime. In such a flow field, the characteristic timescale $\tau$ of the vertical velocity is due to both oscillatory vertical motion and Lagrangian decorrelation. A three-dimensional (3D), high-resolution (1 km horizontal grid spacing, and variable vertical resolution) numerical ocean model is used to simulate a mid-latitude eddy field that is based on hydrography from the western North Atlantic subtropical gyre. The model is set up in a periodic channel $256 \times 320 \mathrm{~km}$ in extent and $1 \mathrm{~km}$ deep. The developed flow field represents characteristics of the subtropical eddy field as described in Freilich and Mahadevan 2019 (Freilich and Mahadevan, 2019). The strongest vertical velocities are at horizontal spatial scales of a few kilometers and occur on the edges of $\sim 100 \mathrm{~km}$ scale mesoscale eddies (Figure 3-2A). We couple this physical model to the simplified nutrient model (3.1) and use a representative regional mean as the equilibrium nutrient profile $N_{0}(z)$ with the nutricline centered at 115 meters (Supp. Fig. C-2). The maximum new production in the model is centered at 115 meters depth, the location of the maximum gradient in the nutrient profile. We vary the uptake rate $\lambda$ between $0.005-50$ day $^{-1}$, which encompasses the observed range of phytoplankton growth rates in nutrient-limited mid-latitude subtropical gyres (Laws, 2013). 
On average, water parcels ascending through the nutracline into the euphotic zone will carry a positive nutrient anomaly compared to surrounding water, while those descending will be nutrient-depleted. When $\lambda \gg \tau^{-1}$ (fast uptake), the nutrient anomaly is small (Fig. $3-2 \mathrm{~B} ; \lambda=1.5$ day $^{-1}$ ) and in phase with $w$ (Fig. 3-2A). The vertical nutrient flux $w N^{\prime}$ at a depth of $115 \mathrm{~m}$ (shown in blue-green shades above the cube in Fig. 3-2B) is small, but positive everywhere, because upward (downward) velocity is correlated with positive (negative) nutrient anomaly. Conversely, for $\lambda \ll \tau^{-1}$ (slow uptake), the nutrient anomaly is large (Fig. $3-2 \mathrm{C} ; \lambda=0.015$ day $^{-1}$ ) and out of phase with $w$ (Fig. 3-2A), resulting in a filamentous distribution of the nutrient as unconsumed nutrient is stirred by the flow. In this case, the vertical flux of nutrient (shown in blue-green shades in Fig. 3-2C) has large values due to the relatively large nutrient anomalies being advected vertically, but the vertical flux is both positive and negative. A negative (downward) flux of nutrient occurs when a positive nutrient anomaly is advected downward before it can be taken up by the slowly growing phytoplankton, or a negative nutrient anomaly is transported upward before it is equilibrated to $N_{0}(z)$. The net flux integrated over the domain is small, as the positive and negative nutrient fluxes cancel each other. The two cases (Fig. 3-2B and C) have a similar net nutrient flux - in one case the local fluxes are small, and in the other, the fluxes are bi-directional and cancel out. The maximum net nutrient flux is achieved (but not shown in the figure) for a value of $\lambda$ that is intermediate between Fig. 3-2B and C. Though several numerical modeling studies (Mahadevan and Archer, 2000) have reported an enhancement in phytoplankton productivity with an increase in vertical velocity, some (Gruber et al., 2011) have also reported a reduction in productivity due to the export of unconsumed nutrient as in Fig. 3-2C.

The spatial distribution of the fluxes depends on the nutrient uptake timescale (Supp. Fig. B-1). With a slow uptake rate $\left(\lambda \ll \tau^{-1}\right)$, the spatial distribution of the nutrient anomaly is heavily influenced by stirring in the horizontal and the cascade to small scales driven by lateral stirring (Abraham, 1998). With a fast uptake rate $\left(\lambda \gg \tau^{-1}\right)$, the nutrient anomaly is present at small spatial scales because the vertical velocity, especially the high frequency component of the vertical velocity, has relatively more small scale variability than the horizontal velocity (Mahadevan and Campbell, 2002; Freilich and Mahadevan, 2021). 


\subsection{Lagrangian description of nutrient flux}

In a nonlinear, eddying oceanic flow field, each water parcel has a unique trajectory. As a water parcel moves upward and downward by 1-100 $\mathrm{m}$ on time scales of days, and then reverses the direction of ascent/descent, it simultaneously moves 1-100 kilometers horizontally. We can isolate the vertical motion of the water parcels by following them in a Lagrangian frame in our 3D flow field while tracking their nutrient concentration and nutrient flux by solving (3.1) on each water parcel trajectory for 14 different values of $\lambda$ in the range $0.005-50$ day $^{-1}$. The time-integrated vertical nutrient flux $\overline{w N^{\prime}}$ along water parcel trajectories is calculated over 30 days as water parcels move through the nutricline.

\subsubsection{Theoretical dependence of flux on biological rate}

Theoretically, we can estimate the time-averaged flux $\overline{w N^{\prime}}$ from $\frac{\partial N^{\prime}}{\partial t}+w \frac{\partial}{\partial z}\left(N_{0}(z)+N^{\prime}\right)=$ $-\lambda N^{\prime}$, if we assume that the mean free path of water mass trajectories, meaning the average magnitude of depth variation, is small relative to variations in the slope of the background nutrient concentration $\frac{d N_{0}}{d z}$. This assumption may introduce errors in some situations, but is valid near equilibrium and for small amplitude perturbations (Supp. Text A.4). We solve for the nutrient anomaly $N^{\prime}$ using the integrating factor method (see Supp. Text A.1 for details). The vertical flux of nutrient is the covariance between the nutrient anomaly and vertical velocity along a given trajectory and is given by the auto-covariance of $w$ weighted by $\lambda$ as

$$
\overline{w N^{\prime}}=\frac{d N_{0}}{d z} \int_{0}^{t} e^{-\lambda(t-s)} w(s) w(t) d s
$$

The nutrient concentration contains a memory of vertical motion on the uptake timescale $\left(\lambda^{-1}\right)$. The vertical velocity fluctuations are averaged over a longer period of time for smaller (as compared to larger) values of $\lambda$.

\subsubsection{Vertical velocity characteristics and biophysical fluxes}

For each model trajectory, we identify the value of $\lambda$ that maximizes the flux $\overline{w N^{\prime}}$. We denote this value as $\lambda_{0}$ Each water parcel trajectory has a full spectrum of vertical velocity frequencies, but the relative contribution of different vertical velocity frequencies is variable 


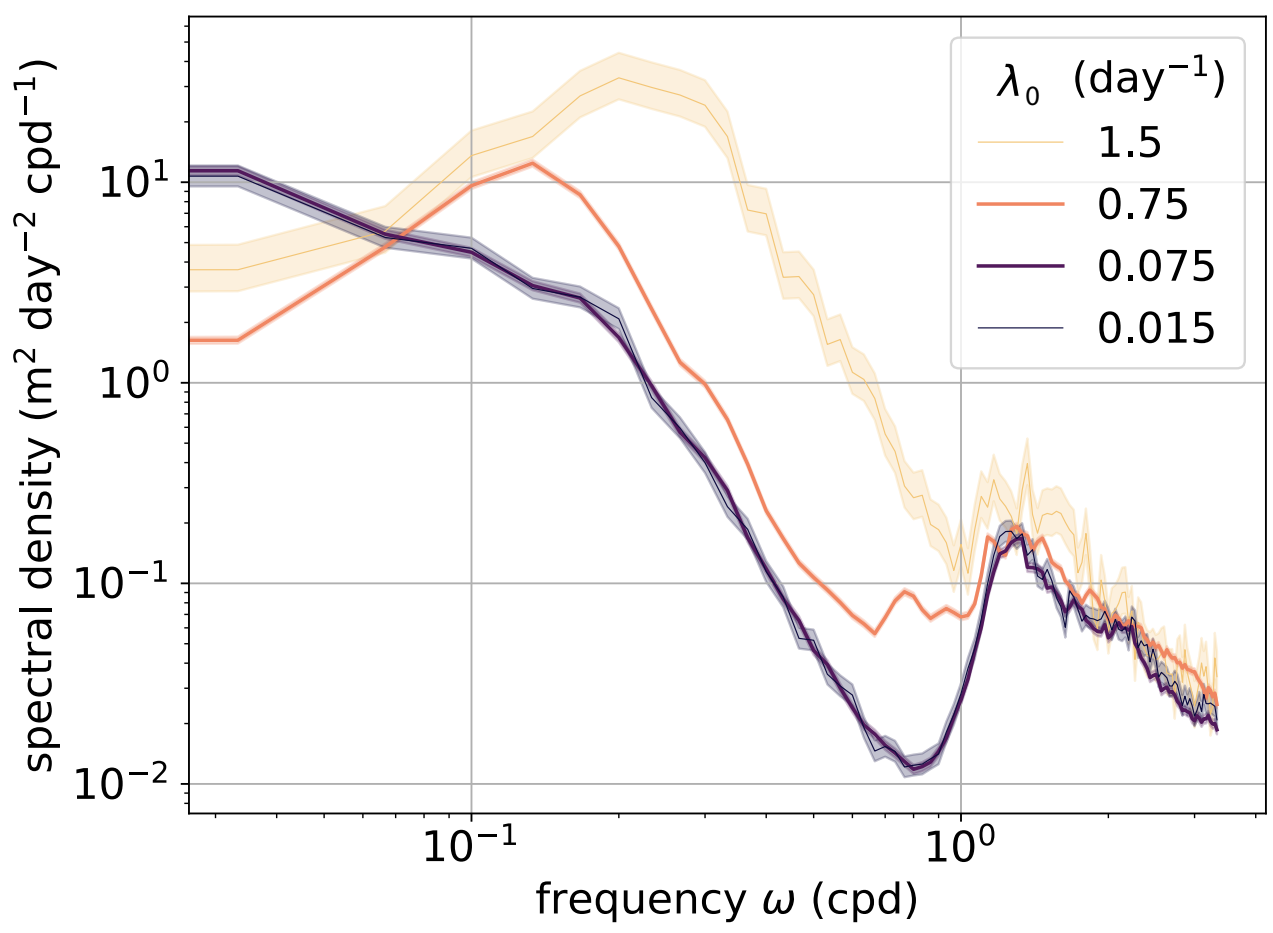

Figure 3-3: Frequency spectra averaged over all Lagrangian particles with a given uptake rate that maximizes the nutrient flux.

across the domain. The Lagrangian frequency spectrum (Fig. 3-3) averaged over all those trajectories on which nutrient flux is maximized for $\lambda=0.075$ day $^{-1}$ (slow uptake) has more power at low frequencies, as compared to the frequency spectrum of trajectories on which nutrient flux is maximized for $\lambda=0.75$ day $^{-1}$ (fast uptake). All trajectories have a peak at the inertial frequency, but the near-inertial oscillations contribute relatively little to the vertical fluxes (Supp. Fig B-1).

When we average across all water parcel trajectories in the 3D model, we obtain a maximum in the average Lagrangian nutrient flux as a function of the uptake rate $\lambda$ (Figure 3-4, orange curve) despite the wide range in the shapes of the Lagrangian spectra. The uptake rate that maximizes the average flux in the $3 \mathrm{D}$ model is $1 / 3$ day $^{-1}$. The corresponding timescale of 3 days is shorter than the turnover time scale of mesoscale eddies in subtropical gyres, but is characteristic of submesoscale dynamics. As a comparison, we calculate the along-trajectory nutrient flux on the full suite of water parcel trajectories according to our theoretical estimate, which is based on the vertical velocity autocovariance (3.3). For 

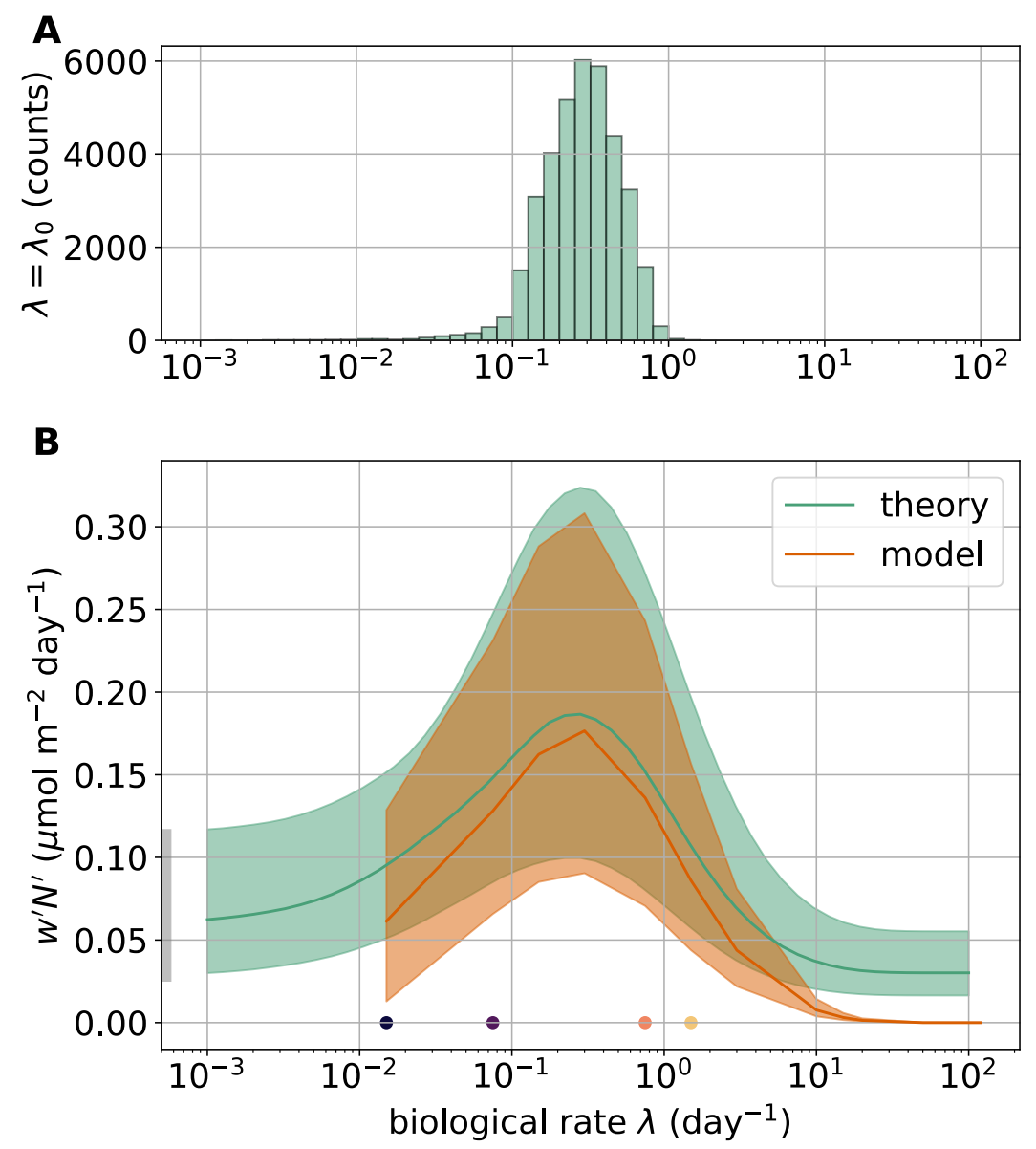

Figure 3-4: (A) Distribution of rates $\lambda$ that maximizes the nutrient flux. (B) Nutrient flux as a function of uptake rate on Lagrangian trajectories (orange curve) and theoretical expectation of the nutrient flux from equation 3.3 (green curve). The solid line is the median of all trajectories; shading shows the interquartile range. The grey bar on the ordinate shows the interquartile range of the vertical eddy diffusion in the absence of biological uptake. The dots on the flux $=0$ axis indicate the uptake rates shown in Fig. 3-3.

any given $\lambda$, there is a range in the nutrient flux achieved through the different individual trajectories due to the difference in the vertical velocity frequency characteristics on individual trajectories. Along each trajectory, the theoretical expectation of the flux and the flux obtained from the 3D model are consistent (Supp. Fig. A-2).

In the absence of biological uptake, the nutrient flux is given by (3.3) with $\lambda=0$. This is the Taylor (1922) (Taylor, 1922) dispersion (Figure 3-4B; grey bar). On average, biological uptake at the rate $\lambda=1 / 3$ day $^{-1}$ results in an enhancement of the vertical nutrient flux to three times the flux with no biological uptake. If the community has a uniform rate of 
0.3 day $^{-1}$, the median flux is $0.177 \mu \mathrm{mol} \mathrm{m}{ }^{-2}$ day $^{-1}$. However, the extreme values of the flux are even larger. If the rate is such that it maximizes the flux locally on each trajectory (Figure 3-5), the median flux is $0.193 \mu \mathrm{mol} \mathrm{m}^{-2}$ day $^{-1}$, a $10 \%$ increase.
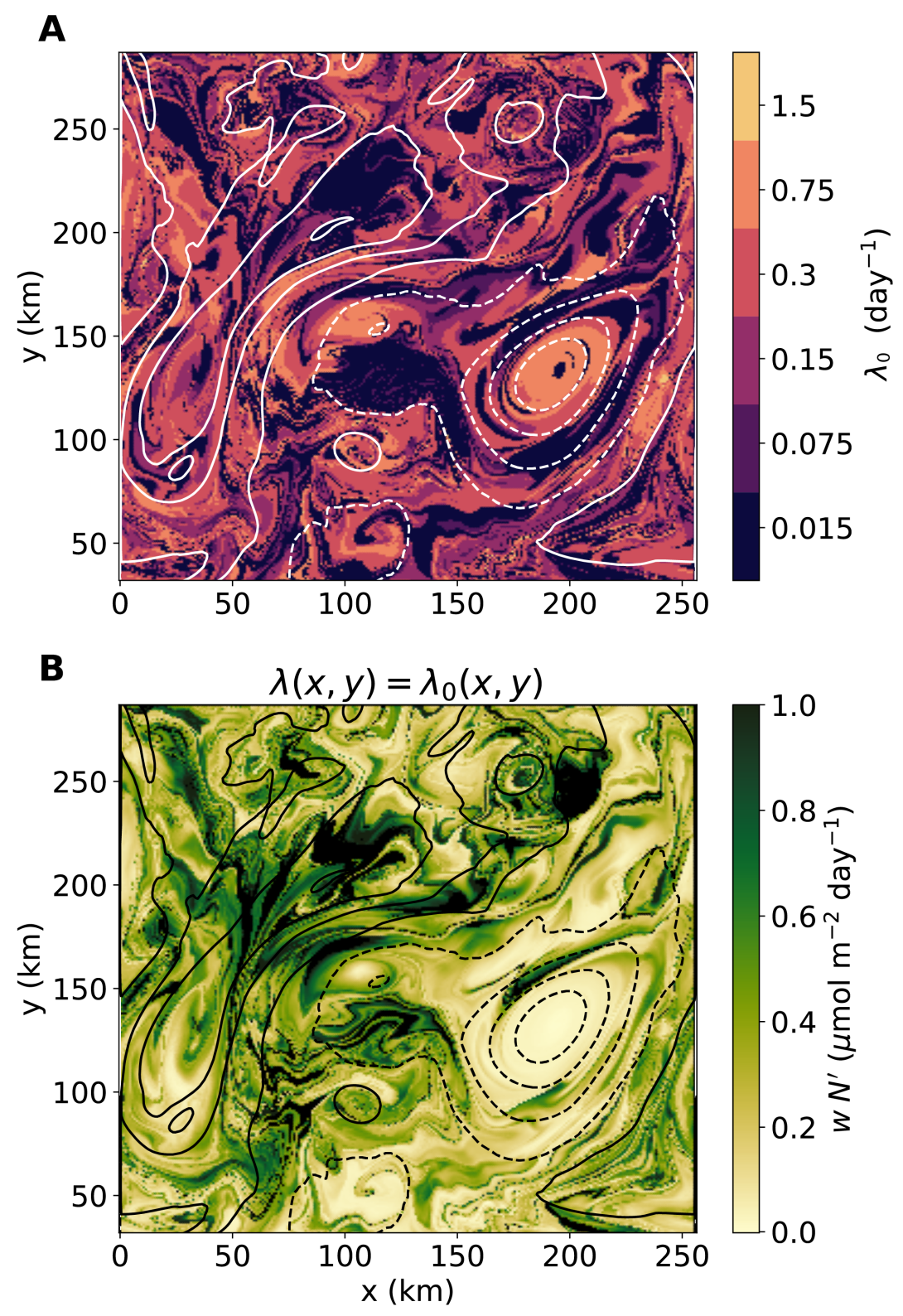

Figure 3-5: (A) Uptake rate that maximizes the nutrient flux for each trajectory plotted at the trajectory origin location. (B) Nutrient flux at the uptake rate that maximizes the flux. The black points are excluded from the analysis in figure 3-4 because they leave the nutricline. The contours are density with the dashed contours being lighter densities.

The difference between a uniform uptake rate and the rate that maximizes the flux 
on the trajectory relates to the variation in the vertical velocity frequency characteristics (Figure 3-3). These characteristics relate to the dynamics of the flow field. A spatial distribution of $\lambda_{0}$ plotted at the origin of each trajectory (Figure 3-5) shows variability that corresponds with the physical features of the flow field. Different features of the flow field favorably support the growth of phytoplankton with uptake rates $(\lambda)$ that match the vertical velocity frequency supported by their own dynamics. We find the nutrient flux to be maximized by small $\lambda$ (slow uptake) on Lagrangian trajectories that originate in relatively stable regions of the flow, while large values of $\lambda$ (fast uptake rates) maximize the nutrient flux on trajectories that are in unstable regions or where eddies interact. The process described here is a mechanism for coexistence of very different biological communities over short spatial scales due to differential growth that can be attributed to small scale variability in the vertical velocity (Perruche et al., 2011). This mechanism differs from lateral stirring of distinct phytoplankton communities, which also results in finescale spatial variability of the community composition (d'Ovidio et al., 2010; Lévy et al., 2015). This framework is also applicable at larger spatial and temporal scales. In global surveys, phytoplankton community structure has also been observed to depend on local rates of resource supply (Marañón, 2015).

\subsection{Discussion}

Observations show that fronts and eddies affect the rate of primary production and the community composition (Letelier et al., 2000; Rodriguez et al., 2001; Sakamoto et al., 2004; White et al., 2007; McGillicuddy, 2016b). At a long-term oceanographic timeseries in the subtropical North Pacific, Station Aloha, there is a six-fold variation in the growth rate in the euphotic zone (Figure 3-6). However, primary production is not linearly correlated with mesoscale eddy variability (Barone et al., 2019; Ferrón et al., 2021). We observe that the average covariance of nutrient anomalies at the base of the euphotic zone and the carbon specific growth rate (Equation 3.2) over the 30 year record has a nonlinear dependence on the growth rate. This correspondence with our theoretical prediction suggests that physical processes exert a strong control on the overall nutrient flux and that vertical motions with 
submesoscale timescales contribute the largest nutrient fluxes. It is notable that a similar biological timescale that results in the maximum flux in our idealized model of a subtropical eddy field emerges as the timescale that maximizes the flux in the North Pacific subtropical gyre. This rate, $\lambda \sim \mathcal{O}\left(0.1\right.$ day $\left.^{-1}\right)$, is also approximately the average growth rate in the North Atlantic subtropical gyre (Goericke and Welschmeyer, 1998). The generation and utilization of nutrient anomalies in the euphotic zone depends on biophysical coupling.
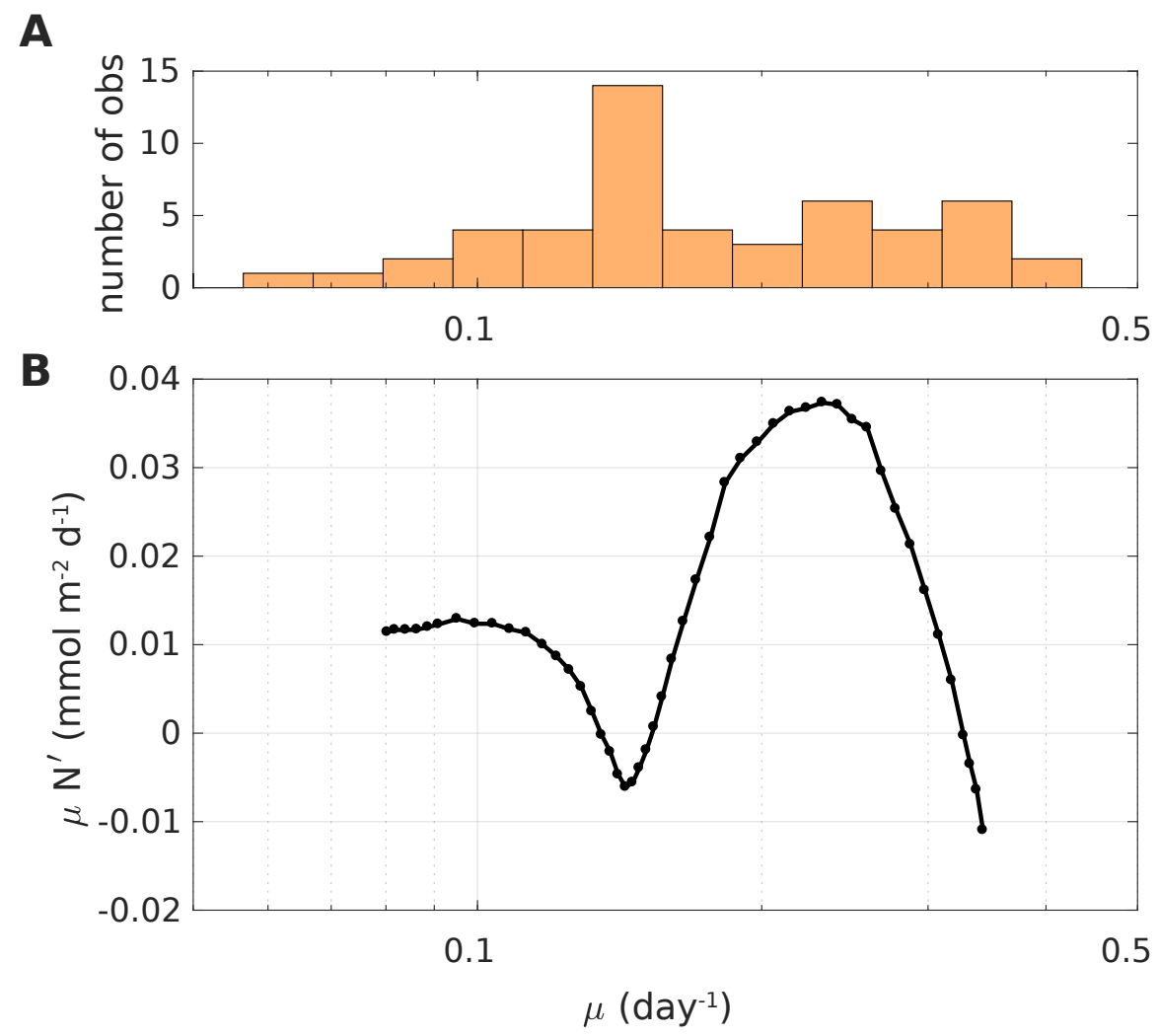

Figure 3-6: (upper) Histogram of carbon-specific growth rate estimated from primary production and cell counts at the Hawaii Ocean Time-series station ALOHA (lower) Temporal average of biological nutrient flux at $120 \mathrm{~m}$ as a function of the carbon specific growth rate at $120 \mathrm{~m}$.

The interrelatedness of the phytoplankton uptake rate, nutrient anomaly, vertical velocity and nutrient supply shown in this model system is applicable to other biogeochemical properties that may be altered by biological processes. The distributions of oxygen, pollutants, and trace elements in the ocean also depend on both biological uptake and physical fluxes, either in the boundary layer or the ocean interior. At large spatial and temporal 
scales, ocean carbon uptake depends on the interaction between relatively slow equilibration and remineralization timescales, water mass formation, and eddies (Mignone et al., 2006; Kwon et al., 2009). On shorter timescales, the magnitude of light-limited primary production in the surface mixed layer is determined by the interaction between mixed layer overturning and the rate of photoacclimation, both of which occur on timescales of less than one day (Dusenberry et al., 2000; Taylor and Ferrari, 2011). This theoretical framework that rigorously defines the relationship between vertical transport characteristics and the biological rate helps to resolve long-standing uncertainties in the role of physical processes in biogeochemical fluxes (Gruber et al., 2011; Liu and Levine, 2021).

The model used here neglects the complexity of biological interactions, which are both nonlinear and occur over multiple timescales. Phytoplankton also have physiological characteristics such as luxury nutrient uptake among diatoms that can decouple productivity from nutrient uptake. In the case of nonlinear reactions the theory outlined here will still apply but analytic solutions are less tractable. In general with a nonlinear biological function $f(N)$ in equation 3.1 the magnitude of the biogeochemical anomaly itself will depend nonlinearly on the biological rate and the timescale may be determined by nonlinear processes such as ecologically-driven oscillations (Franks, 2001; Neufeld, 2012), which will affect the nature of the dependence of the vertical flux magnitude on the biological rate. Vertical fluxes may also be affected by vertical variations of both biological rates and the vertical velocity magnitude (Kahru, 1983). In a multi-species system, the inclusion of cross-fluxes between components will be necessary and will be a source of spatial and temporal variance (Abraham, 1998; Falkowski et al., 1998; Martin and Pondaven, 2003; Morison et al., 2020). Observed variations in community composition may also be a result of ecological interactions but may not alter the net community production (Malone et al., 1993; Giovannoni and Vergin, 2012). Future work could address the implications of the dependence of flux on biological rate for changes in community composition at the submesoscale.

Though there is not a clear separation between the mesoscale and submesoscale, but rather a continuum in which the integral timescale of the vertical velocity is affected by eddies, the $\lambda_{0}^{-1} \approx 3$ days timescale that emerges from among the many trajectories in our model of an eddying flow field is within the range of timescales of up- and down-ward 
motion associated with sub-inertial submesoscale dynamics, corroborating the importance of its contribution for nutrient supply. Submesoscale features are small-scale which makes them difficult to observe directly. Nutrient fluxes and net community production may be underestimated if submesoscale features are not included. Due to the nonlinearity of flux, the mean state will be different from one that resolves the small-scale dynamics (Liu and Levine, 2021).

A practical implication of this work for modeling studies is the need to account for what physical timescales are included in a model when parameterizing biology. Studies that compare the effect of different model resolutions on primary productivity while holding the biological rates constant may find that the observed effect is sensitive to the biological timescales that are selected due to alterations in the mean nutrient gradient (Lévy et al., 2012b; Balwada et al., 2018; Resplandy et al., 2019). If the vertical velocity processes with timescales on the order of the biological timescales are underrepresented, then there may be a mismatch between the biological and physical parameterizations. Nutrient uptake timescales most closely match the timescales of mesoscale and submesoscale processes, which are not represented in most global models that are used to make projections about climate change. These fluxes are often parameterized as a turbulent diffusion (Denman and Gargett, 1983). However, because the timescale of the diffusion feeds back on the nutrient gradient, the biological rate affects the eddy diffusivity coefficient and a diffusive parameterization may not appropriately represent the fluxes, especially when the physical and biological timescales are similar (Smith et al., 2016). As a consequence, the projected changes in ocean biogeochemcial cycles may be highly sensitive to the relationship between the biological and physical models (Löptien and Dietze, 2019; Brett et al., 2021). Instead a Lagrangian flux parameterization as suggested here may aid both analysis and parameterization (Plumb, 1979). Better understanding of the frequency characteristics of submesoscale dynamics (Torres et al., 2018; Callies et al., 2020) could improve diagnosis of biogeochemical fluxes and ecological community composition. 


\subsection{Conclusions}

The vertical flux of nutrients is modulated by the interaction between phytoplankton growth rate (sinks and sources that alter the nutrient concentration), and the frequency of the vertical velocity. Even with linear biological reactions, the interaction between biological and physical processes results in a nonlinear dependence of the biogeochemical flux on the biological rate. At the same time, the vertical flux influences the growth and distribution of phytoplankton, suggesting that the variable rate of nutrient supply in an oceanic eddy field can structure the phytoplankton communities.

\subsection{Methods}

\subsubsection{Physical model}

We simulate an eddying flow field using the Process Study Ocean Model (PSOM) (Mahadevan et al., 1996b,d). The numerical model is initialized with a horizontal buoyancy gradient generated by altering a central temperature and salinity from an Argo profile that was taken in the subtropical gyre on December 11,2015 . The center of the model domain is at $35^{\circ} \mathrm{N}$. The model is forced with differential heating and cooling to maintain the initial buoyancy gradient. The model is a periodic channel that is $256 \mathrm{~km}$ in the $x$ direction, $320 \mathrm{~km}$ is in the $(y)$ direction, and $1000 \mathrm{~m}$ deep. The horizontal resolution is $1 \mathrm{~km}$. In the vertical, the model has a stretched grid with spacing that ranges from $3 \mathrm{~m}$ near surface to $32 \mathrm{~m}$ at the bottom. The time step for numerical integration is 432 seconds. The model is analyzed after it has spun up for 120 days, by which time an eddy field is well-developed and the kinetic energy is in equilibrium. The analysis period is 60 days.

\subsubsection{Biological model}

We model nutrient concentration $N$ using equation 3.1 with 14 distinct values of the uptake rate $\lambda$ on Lagrangian particles and as an offline tracer using a forward Euler scheme. The

advection of biological tracers is performed offline using the model advection routines and the advection of Lagrangian particles is performed using an implementation of the Doos 
and deVries algorithm in Python. We use a hyperbolic tangent function as the background nutrient concentration, which provides a nutricline, or localized gradient in the nutrient profile, and generates a deep biomass maximum. The width of the model nutricline is set by the approximate observed depth range of the deep chlorophyll maximum in the subtropical North Atlantic. While this is a good model of the nitracline, it does not represent the deep nitrate concentration, which increases with depth (and increases with density). However, we assume no production takes place at depth which requires this equilibrium profile to have a slope of zero at depth because we have assumed a constant growth rate.

\subsubsection{Observations}

We compute the carbon specific growth rate using observations from the Hawaii Ocean Timeseries. There are 31 years of monthly observations at station ALOHA, which is located at $22^{\circ} 45^{\prime} \mathrm{N}, 158^{\circ} 00^{\prime} \mathrm{W}$. Primary production is measured using ${ }^{14} \mathrm{C}$ incubations for 12 hours at a range of depths within the euphotic zone. The primary production $(\Delta C)$ is computed as the difference between light and dark incubations. The carbon specific growth rate is computed as

$$
\mu=\ln \frac{\mathrm{C}+\Delta C}{\mathrm{C}}
$$

(Pérez et al., 2006). The phytoplankton carbon (C) is estimated from flow cytometric cell counts using the average conversion factors from cells to carbon from Buitenhuis et al. (2012). The nutrient anomaly is computed as the deviation from the monthly average nutrient concentration at each depth. The production $\mu N^{\prime}$ is estimated by computing a forward then backward 15 point moving mean of $\mu N^{\prime}$ evaluated at $120 \mathrm{~m}$ sorted by depth integrated $\mu$. 


\title{
Chapter 4
}

\section{Coherent pathways for subduction}

\section{from the surface mixed layer at ocean}

\section{fronts}

\begin{abstract}
In frontal zones, water masses that are tens of kilometers in extent with origins in the mixed layer can be identified in the pycnocline for days to months. Here, we explore the pathways and mechanisms of subduction, the process by which water from the surface mixed layer makes its way into the pycnocline, using a submesoscale-resolving numerical model of a mesoscale front. By identifying Lagrangian trajectories of water parcels that exit the mixed layer, we study the evolution of dynamical properties from a statistical standpoint. Velocityand buoyancy-gradients increase as water parcels experience both mesoscale (geostrophic) and submesoscale (ageostrophic) frontogenesis and subduct beneath the mixed layer into the stratified pycnocline along isopycnals that outcrop in the mixed layer. Subduction is transient and occurs in coherent regions along the front, the spatial and temporal scales of which influences the scales of the subducted water masses in the pycnocline. An examination of specific subduction events reveals a range of submesoscale features that support subduction. Contrary to the forced submesoscale processes that sequester low potential vorticity $(\mathrm{PV})$ anomalies in the interior, we find that PV can be elevated in subducting water masses. The rate of subduction is of similar magnitude to previous studies ( $\sim 100 \mathrm{~m} /$ year), but the pathways that are unraveled in this study, along with the Lagrangian evolution of properties on water parcels, emphasize the role of submesoscale dynamics coupled with mesoscale frontogenesis. ${ }^{1}$
\end{abstract}

\footnotetext{
${ }^{1}$ This chapter is published as Freilich, M and Mahadevan, A (2021). Coherent pathways for subduction from the surface mixed layer at ocean fronts. Journal of Geophysical Research: Oceans, e2020JC01702.
} 


\subsection{Introduction}

The exchange of properties between the ocean and atmosphere, including heat, carbon, and oxygen, is affected by subduction, which is the transport of water from the surface mixed layer into the stratified pycnocline. Subduction ventilates the pycnocline, affects the water mass characteristics of the interior, and impacts the ocean's biogeochemistry. The seasonal transformation of the mixed layer and the large-scale circulation (Nurser and Marshall, 1991; Lévy et al., 2013) leads to subduction through diabatic processes. In addition, subduction occurs along sloping isopycnals at fronts, where mixed-layer enhanced submesoscale processes enhance vertical advection on horizontal scales of 0.1-10 km (Bosse et al., 2015; Omand et al., 2015; Klymak et al., 2016; Stanley et al., 2017; Wenegrat et al., 2018; Freilich and Mahadevan, 2019).

Large vertical velocities in the mixed layer can arise from a range of submesoscale processes (Haine and Marshall, 1998; Mahadevan and Tandon, 2006; McWilliams, 2016). These include mixed layer instability (Fox-Kemper et al., 2008), submesoscale frontogenesis in the mixed layer (Thomas et al., 2008; Barkan et al., 2019), and boundary forced submesoscale dynamics, such as non-linear Ekman pumping and surface-forced symmetric instability (Thomas et al., 2013). Submesoscale flows generate vertical velocities of $\mathcal{O}(100) \mathrm{m}$ $\mathrm{d}^{-1}$ (Fox-Kemper et al., 2008; Mahadevan et al., 2010) and are characterized by large, $O(1)$, Rossby number, and low (also $O(1)$ ) Richardson number. The downward velocities due to submesoscale dynamics are larger in magnitude and concentrated in smaller regions than the upward velocities (Shcherbina et al., 2015). Within the mixed layer, submesoscale flows coexist with boundary layer turbulent motion, which can generate vertical velocities of $\mathcal{O}(1000) \mathrm{m}_{-} \mathrm{d}^{-1}$ within the mixed layer and is resolved by large eddy simulations (LES) (Skyllingstad et al., 2017; Verma et al., 2019) and observed with Lagrangian instruments (D'Asaro et al., 2018).

Submesoscale instabilities are generally enhanced in the mixed layer, but the vertical density gradient (stratification) at the base of the mixed layer is typically greater than in the pycnocline and vertical transport across the mixed layer base is inhibited. However, submesoscale dynamics also has an influence below the surface mixed layer. Symmetric instability 
mixes momentum and tracers along isopycnal surfaces, often reaching below the mixed layer (Thomas et al., 2013) and leading to exchange of tracers between the surface and pycnocline (Smith et al., 2016; Erickson and Thompson, 2018; Archer et al., 2020). Submesoscale mixed layer eddies can also barotropize and induce along-isopycnal stirring in the pycnocline (Badin et al., 2011). Furthermore, recent observational and modeling studies have shown that large vertical buoyancy fluxes within the pycnocline have characteristic spatial and temporal scales of submesoscale processes that may be attributable to geostrophic frontogenesis (Yu et al., 2019; Siegelman et al., 2020). These studies raise the possibility that surfaceenhanced submesoscale dynamics may influence the pycnocline directly through water mass exchange when there is a non-zero vertical velocity across the mixed layer base.

There are two main processes that could adiabatically subduct water parcels from the mixed layer. The first is restratification of the mixed layer. As the mixed layer restratifies due to either heat fluxes or the slumping of isopycnals (Fox-Kemper et al., 2008; Mahadevan et al., 2012), some water parcels end up beneath the newly reformed mixed layer. The second process, which will be the focus of this paper, involves movement along sloping density surfaces. If a density surface from the pycnocline outcrops into the mixed layer, then water parcels can move adiabatically along that surface from the mixed layer into the interior (Stommel, 1979; Nurser and Marshall, 1991; Gebbie, 2007; MacGilchrist et al., 2017; Canuto et al., 2018).

Frontal regions, where sloping isopycnals from the pycnocline outcrop in the surface mixed layer at the mesoscale and submesoscale, offer an advective pathway for the exchange of water across the base of the mixed layer (Ramachandran et al., 2014). Frontogenesis, which is the intensification of lateral buoyancy (or density) gradients at fronts, dynamically induces subduction (and upwelling) along the sloping isopycnal surfaces of the front and does not necessarily involve the shoaling of the mixed layer. Submesoscale instabilities can occur along strong mesoscale currents (Thomas and Joyce, 2010; Gula et al., 2016), enhancing vertical motion and generating an asymmetry in the up-/down-ward motion, with subduction being more localized and intensified than upwelling.

Our objective is to study the dynamical processes and pathways through which the mixed layer and pycnocline connect in a frontal flow field and to provide insight into the subduc- 
tion mechanisms when the mixed layer is not undergoing restratification. We use numerical modeling to interrogate the coupling between mesoscale and submesoscale processes along a strong mesoscale frontal meander with a 50-70 m deep mixed layer. The model is representative of the front between Atlantic and Mediterranean waters in the Alborán Sea, where the subduction of biogeochemical tracers was observed along a mesoscale frontal meander (Pascual et al., 2017; Ruiz et al., 2019). We do not force the model with winds or strong buoyancy fluxes at the surface, which would generate boundary layer turbulence with large vertical velocities in the mixed layer. Further, we do not resolve processes at scales smaller than $500 \mathrm{~m}$, such as symmetric instability. Instead, we study how unforced frontogenesis leads to subduction by following the evolution of dynamical and kinematic properties as water subducts out of the mixed layer along isopycnals.

Studying frontogenesis in the Lagrangian frame has several advantages. Firstly, the time history of processes on a water parcel as they evolve over a few days provides information about subduction. We are interested in transport, or the integrated motion over a few days, and not vertical velocity itself, which contains waves and other high frequency motions. Secondly, while we are interested in the downward motion of water, the along-front flow is faster by 3-4 orders of magnitude. Hence, snapshot views of frontal cross-sections do not convey where the subducting water originates, or where it is headed. Thirdly, subduction does not occur everywhere along the front and to identify where it occurs, and on what spatial scales, we need to tag the water parcels that subduct. Following the advecting water parcels as they subduct allows us to construct a time history of the mechanisms. We define subduction as occurring when water parcels leave the mixed layer and travel at least $5 \mathrm{~m}$ below the mixed layer base into the pycnocline. By identifying the locations where water leaves the mixed layer, we examine the spatial scales of the process. The evolution of dynamical properties along water parcel trajectories reveals how frontogenesis acts to subduct water, but that there are a wide variety of subduction pathways along which the dominant frontogenetic and frontolytic factors can differ (Thomas, 2008; Barkan et al., 2019). To begin, we discuss observations of subduction at the front between fresher (less dense) Atlantic water and saltier (denser) Mediterranean water from the Alboran Sea. Next, we offer some theoretical background based on which we develop hypotheses about the subduc- 
tion processes. We then describe our idealized model and the Lagrangian methodology used for analysis. In our results, we present a statistical view, as well as a description of specific subduction pathways. Finally, we offer a discussion of our results and their implications in a broader context.

\subsection{Observational motivation}

Observations show that coherent subducted water masses are ubiquitous at strong fronts (Thomas and Joyce, 2010; Pascual et al., 2017). In March and April of 2019 we embarked on an observational campaign to study the pathways of vertical transport of natural tracers from the surface ocean to the interior. We conducted hydrographic surveys on board the N/O Pourquoi Pas? from March 28-April 11 at strong mesoscale and submesoscale fronts in the Alborán Sea which is the westernmost part of the Mediterranean (Mahadevan et al., 2020). We measured vertical profiles of conductivity, temperature, and pressure using an Oceansciences underway CTD system in tow-yo mode at a spatial resolution of around $1 \mathrm{~km}$ while the ship was transiting across fronts (Johnston et al., 2019). Ocean velocities was measured from a $150 \mathrm{kHz}$ vessel-mounted ADCP.

The Western Alborán Gyre is formed by the mesoscale meander of the baroclinically unstable front between the saltier resident Mediterranean water and the fresher Atlantic water that enters through the strait of Gibralter (Figure 4-1A; longitudes 3.5W to 5.5W). We traversed across the front several times, and Figure 4-1B shows, as an example, the hydrography from a section across the northern edge of the Western Alborán Gyre. The density gradient at the front is mostly due to salinity. Variations in temperature along a density surface are indicative of a different water mass that has intruded through subduction and undergone stirring. There are multiple anomalously warm intrusions that extend from the surface into the pycnocline along isopycnals. Though these intrusions are visible in the cross-front direction, they are also expected to be moving rapidly in the along-front direction (out of the page in Figure 4-1B). Casts from a shipboard CTD show that these temperature anomalies are associated with unusually high fluorescence and low apparent oxygen utilization for the depth at which they occur, adding support to the idea that these are recently subducted, 

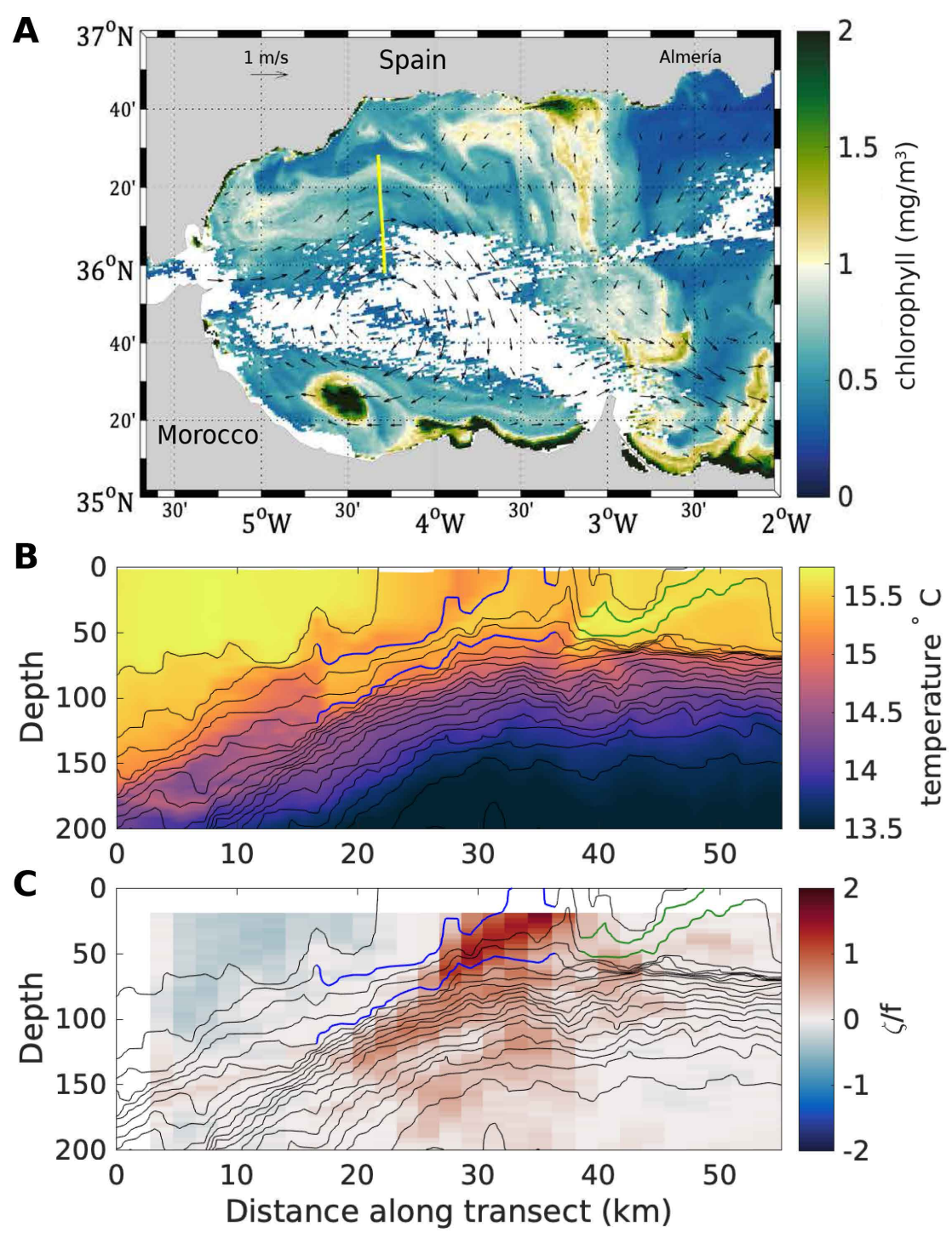

Figure 4-1: (a) MODIS Aqua satellite image of chlorophyll in the Alborán Sea on March 28, 2019. The geostrophic currents from AVISO (arrows) show the anticyclonic Western Alborán Gyre. The chlorophyll (in color) shows the influence of frontal dynamics at the gyre edge with frontal waves on the northern edge of the gyre. The CALYPSO cruise on the $N / O$ Pourquois Pas? surveyed the front transiting from south to north along the yellow line on March 30, 2019. (b) Transect of temperature (color) from an underway CTD with density contours at intervals of $0.1 \mathrm{~kg} / \mathrm{m}^{3}$. Subducted water masses are identified by temperature variations along an isopycnal surface. Two warm intrusions are highlighted by demarcating their bounding isopycnals in blue. (c) Transect of relative vorticity computed from the along-track gradient of the horizontal velocity normal to the ship section. Velocity measurements are from a vessel-mounted ADCP. 
photosynthetically productive, water masses. The along-track vorticity, which, despite neglecting cross-track gradients, is a good estimate of the relative vertical vorticity (because the ship traverses across the front), has surface-intensified cyclonic values at the center of the front (Figure 4-1C). On the dense side of the front, the vorticity is cyclonic, reaching $\approx 2 f$ at the center of the front, while on the light side of the front the vorticity is weakly anticyclonic.

The surface chlorophyll image shows high chlorophyll on the gyre edge that forms frontal waves or cusp-like features (Figure 4-1A), one of which is sampled by the ship transect. The feature on the northern flank of the front is found to be an eddy, $10 \mathrm{~km}$ in diameter and $70 \mathrm{~m}$ deep (Figure 4-1B). There is an anomalously warm intrusion of water wrapped around the submesoscale eddy on the dense side of the front, which extends from the surface to the upper pycnocline (Figure 4-1B). Despite the light core, this eddy has cyclonic vorticity (Figure 4-1C); the cusps in the chlorophyll image also suggest cyclonic rotation. A similar feature is found in the modeling study that follows. Another temperature intrusion is co-located with the high relative vorticity at the center of the front, where density surfaces from the upper pycnocline outcrop (Figure 4-1B,C). The intrusion extends $100 \mathrm{~m}$ in the vertical and $30 \mathrm{~km}$ in the north-south direction along density surfaces. On the deep end of this temperature intrusion, the feature has anticyclonic vorticity and reduced stratification. These observations reveal pathways of natural tracers, or temperature anomaly on an isopycnal, from the surface mixed layer to the upper pycnocline that are coherent over scales of tens of kilometers. The vertical displacement associated with these features is on the order of 100 meters. Unlike some previous observations that report subduction of low potential vorticity (PV) water, these subducted features are associated with strong cyclonic vorticity and are moderately stratified (Beaird et al., 2016; Archer et al., 2020). We use a process study model to examine the role that unforced frontal dynamics might have played in these observed subduction events, elaborate on the dynamical mechanisms of subduction, and describe the role of along-front variability in subduction from the surface mixed layer to the interior. 


\subsection{Theoretical background}

The lateral buoyancy (and density) gradient at ocean fronts can be intensified through the mechanism of frontogenesis (McWilliams, 2021). Here, buoyancy $b \equiv-\frac{g}{\rho_{0}}\left(\rho-\rho_{0}\right)$, where $\rho$ is the potential density, $\rho_{0}=1027 \mathrm{~kg} \mathrm{~m}^{-3}$ is a reference potential density, and $g$ is the acceleration due to gravity. The vertical and horizontal buoyancy gradients are denoted by $N^{2}=b_{z}$ and $M^{2}=\left|\nabla_{h} b\right|$. Treating buoyancy, $b$, as a tracer, the Lagrangian rate of change of the horizontal buoyancy gradient $\nabla_{h} b$ can be expressed as

$$
\frac{D}{D t} \nabla_{h} b=\underbrace{\left(-u_{x} b_{x}-v_{x} b_{y},-u_{y} b_{x}-v_{y} b_{y}\right)}_{\mathbf{Q}}-N^{2} \nabla_{h} w+\kappa_{H} \nabla_{h}^{2} \nabla_{h} b+\kappa_{V} \partial_{z z} \nabla_{h} b,
$$

where $\nabla_{h}$ is the horizontal gradient operator in the $x-y$ plane and $D / D t \equiv \partial_{t}+u \partial_{x}+v \partial_{y}+$ $w \partial_{z}$ is the material derivative. Here, $\kappa_{H}$, the horizontal diffusivity and $\kappa_{V}$, the vertical diffusivity, are treated as homogeneous and constant. The vector $\mathbf{Q}$ on the right hand side of (4.1) is the tendency of advection to strengthen or weaken buoyancy gradients in the $x$ and $y$ directions and can be decomposed into geostrophic and ageostrohic contributions $\mathbf{Q}=\mathbf{Q}_{\mathbf{g}}+\mathbf{Q}_{\mathbf{a}}$ by using the respective gesotrophic or ageostrophic components of the horizontal velocity $(u, v)=\left(u_{g}, v_{g}\right)+\left(u_{a}, v_{a}\right)$. The square of the frontogenetic tendency (a scalar quantity) is given by

$$
\frac{1}{2} \frac{D}{D t}\left|\nabla_{h} b\right|^{2}=\underbrace{\mathbf{Q}_{\mathbf{g}} \cdot \nabla_{h} b}_{\text {geostrophic }}+\underbrace{\mathbf{Q}_{\mathbf{a}} \cdot \nabla_{h} b}_{\text {ageostrophic }}-\underbrace{N^{2} \nabla_{h} w \cdot \nabla_{h} b}_{\text {vertical }}+\underbrace{\kappa_{H} \nabla_{h}^{2} \nabla_{h} b \cdot \nabla_{h} b}_{k_{h}}+\underbrace{\kappa_{V} \partial_{z z} \nabla_{h} b \cdot \nabla_{h} b}_{k_{v}} .
$$

The large-scale straining that intensifies buoyancy gradients disrupts the thermal wind balance and generates ageostrophic circulation in the vertical plane (Hoskins and Bretherton, 1972). The resulting vertical velocity, $w$, can be diagnosed from the observed frontogenetic strain (Hoskins et al., 1978) using the Omega equation

$$
N^{2} \nabla_{h}^{2} w-f_{0} \partial_{z z} w=2 \nabla_{h} \cdot \mathbf{Q},
$$

where $f_{0}$ is a reference Coriolis parameter. For a more detailed derivation see Section 13.3 
of (Hoskins and James, 2014). In the quasigeostrophic formulation of the Omega equation, $\mathbf{Q}=\mathbf{Q}_{\mathbf{g}}$ contains only geostrophic velocities $\left(\mathbf{u}_{\mathbf{g}}\right)$, so the ageostrophic velocity and the vertical velocities are forced by only geostrophic straining. The lack of a feedback from ageostrophic velocities generated by frontogenesis causes both cyclonic and anticyclonic vorticity increase at the same rate and results in symmetric upwelling and downwelling (Hoskins and Bretherton, 1972), both of which are not true at the oceanic submesoscale (Shcherbina et al., 2015).

As the relative vorticity increases, the quasigeostrophic Omega equation fails to accurately diagnose vertical velocities. The semigeostrophic Omega equation includes a feedback between the ageostrophic velocity and the frontal intensity by allowing for advection of buoyancy and geostrophic velocities by the combined geostrophic and ageostrophic velocities. The non-linear feedback on the relative vorticity is evident from the Lagrangian rate of change of the absolute vorticity. Assuming adiabatic dynamics, the rate of change of the vertical component of the relative vorticity $(\zeta)$ is dominated by the vortex stretching and tilting terms on the right hand side of

$$
\frac{\mathrm{D}(f+\zeta)}{\mathrm{D} t}=(f+\zeta) \frac{\partial w}{\partial z}+\frac{1}{\rho^{2}}\left(\frac{\partial \rho}{\partial x} \frac{\partial p}{\partial y}-\frac{\partial \rho}{\partial y} \frac{\partial p}{\partial x}\right)
$$

At surface convergences (positive $w_{z}$ ), the near surface vertical velocity is downwards (negative) and the relative vorticity increases exponentially. However, at divergences (negative $\left.w_{z}\right)$, the absolute vorticity $(f+\zeta)$ decreases and approaches zero, which slows the rate of decrease of relative vorticity. In addition, symmetric instability limits the relative vorticity to $\zeta \geq-f$.

The vertical structure of the vertical velocity depends on the stratification, the vorticity, and PV, which combines both buoyancy and velocity gradients. Both the stratification and PV gradients are typically large at the base of the mixed layer. PV and vorticity gradients are typically large at fronts. The ageostrophic overturning diagnosed by the semigeostrophic Omega equation is skewed with larger magnitude downward velocities and larger magnitude cyclonic vorticity than the quasigeostrophic solution. (Hoskins, 1982). This can be intuitively understood through a transformation into geostrophic coordinates, which compresses 
the horizontal scale of the ageostrophic overturning and aligns the upwelling branch of the overturning with isopycnal surfaces. An alternative vertical velocity diagnostic equation, the Eliassen-Sawyer equation (Eliassen, 1949; Sawyer, 1956), which is also an elliptic equation forced by the $\mathbf{Q}$ vector, reveals the links between vertical velocity and both the relative vorticity and PV. Vertical velocity is enhanced in regions with low PV, and when the PV is constant, in regions with cyclonic relative vorticity due to reduced horizontal length scales (Thomas, 2008).

As a water parcel is transported by the ageostrophic circulation from the surface mixed layer into the stratified interior, the dynamical properties change on the water parcel. For example, $N^{2}$ on the water parcel increases and the relative vorticity on the water parcel must decrease ("vortex squashing") to conserve PV (Gent and Mcwilliams, 1990; Wang, 1993; Spall, 1995). This ignores the horizontal components of the PV, $q_{h}$, which may be significant in areas with strong lateral density gradients. If $q_{h}$ becomes increasingly negative during subduction, then $\zeta$ would not decrease as much as would be expected from the vertical component alone.

The quasi- and semi-geostrophic theories outlined above are two dimensional and neglect along front curvature. However, the shape of the jet affects the frontogenetic tendency (McWilliams et al., 2019; Buckingham et al., 2021). At submesoscale fronts, the curvature can be of the same scale as the cross-frontal scale, necessitating analysis of three-dimensional processes. Additionally, frontogenesis is classically studied in cases with a deformation flow, but in a strong mesoscale front, the horizontal shear can also contribute to frontogensis (McWilliams, 2021). Moreover, diabatic processes and mixing are particularly important at the sharp fronts that are present at submesoscales (Giordani et al., 2006).

\subsection{Methods}

A three-dimensional numerical model is used to explore the dynamical mechanisms for sub-

duction from the mixed layer into the pycnocline in the absence of strong surface forcing (wind stress or cooling). Our strategy is to follow water parcels and track their proper- 


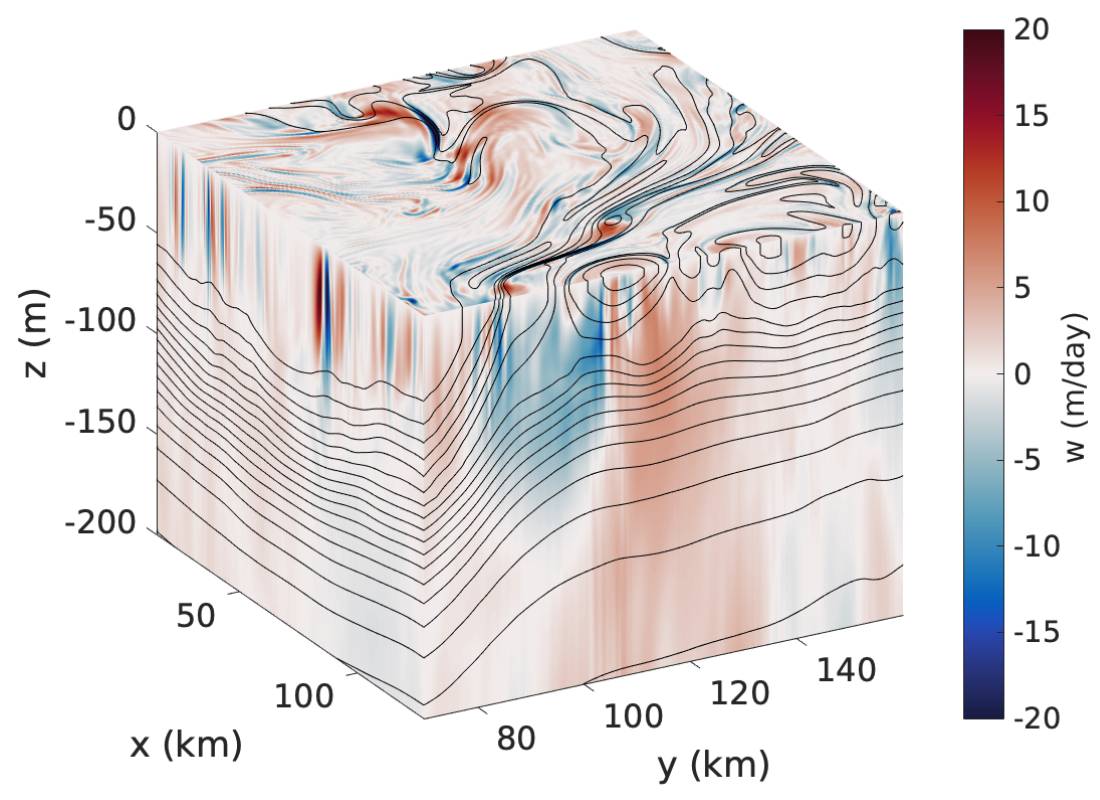

Figure 4-2: Vertical velocity with density contours on day 47.5. The vertical velocity that is shown at the surface is the 5 meter vertical velocity. Vertical velocity is shown in meters/day, with a saturated color scale.

ties as they are subducted. By analyzing the kinematic and hydrographic properties in the Lagrangian frame as water parcels are subducting, we aim to statistically identify the characteristics and evolution of different subduction events.

\subsubsection{Model set up}

We simulate a front using the non-hydrostatic Process Study Ocean Model (PSOM) (Mahadevan et al., 1996c,a; Mahadevan, 2020) in a zonal periodic channel. In the meridional direction, the model is initialized with the observed pycnocline structure of the mesoscale front on the edge of the Eastern Alborán Gyre in the Western Mediterranean and a 5070 meters deep mixed layer, typical of late-winter and early-spring conditions. The initial condition has a small-amplitude meander with one wavelength in the zonal direction to nudge the model to develop the large-scale meandering structure that is observed in the Alborán Sea. The model domain is centered at $36.9^{\circ} \mathrm{N}$. The inertial period is 20 hours.

The model domain extends $128 \mathrm{~km}$ in the (periodic) $x$-direction and $206 \mathrm{~km}$ in the $y$ direction (with closed walls) and $1000 \mathrm{~m}$ in depth. The horizontal resolution is 500 meters, 
with a stretched grid in $y$ that attains a spacing of $2 \mathrm{~km}$ within $40 \mathrm{~km}$ of the southern and northern solid boundaries. There are 64 vertical levels on a stretched grid with grid spacing ranging from $0.5 \mathrm{~m}$ at the surface to $54 \mathrm{~m}$ at depth. The model timestep is 108 seconds. The horizontal diffusion is $1 \mathrm{~m}^{2} / \mathrm{s}$. The vertical diffusion has a constant value of $10^{-5} \mathrm{~m}^{2} / \mathrm{s}$. The model has a flat bottom and a linear bottom drag of $10^{-4} \mathrm{~m} / \mathrm{s}$. The model is forced with weak cooling at a rate of $15 \mathrm{~W} / \mathrm{m}^{2}$ at the surface to maintain the mixed layer. The density is adjusted to a stable state by convective adjustment.

\subsubsection{Particle tracking}

Particle trajectories are used to identify subduction locations and study the evolution of dynamical properties along water parcel trajectories. Particles are advected offline from the model integration of momentum using an implementation of the (Vries and Döös, 2001) particle advection algorithm in Python (Dever and Essink, 2020). The particles are advected using instantaneous velocity fields from the three-dimensional model saved every 3 hours and interpolated linearly to intermediate times. Particle trajectories integrated offline for 10 days (with 3-hourly model output) do not differ significantly from those calculated online in the model. For our study, we seed 12,700 each day at a spacing of $1 \mathrm{~km}$ at 5 meters depth from model days 44 to 62 . The particles are seeded across the whole domain. A total of 228,600 particles are used in this study. Tracers and velocities are interpolated from the model grid onto the particle positions using tri-linear interpolation. All gradients are computed on the model grid and then interpolated onto the particle positions.

\subsubsection{Tracers}

Similar to the particles, we advect 2 tracers offline with the model's advection routine and a time step of 108 seconds. The 3-hourly model output that was saved is interpolated in time onto the time step of the tracer advection model.

A mixed layer tracer is used to diagnose the subduction rates and validate the particle results (Figure S1). This tracer is initialized with a value of 1 in the mixed layer and 0 outside the mixed layer. At every time step, the tracer concentration is instantaneously restored to 1 in the local mixed layer, which is recomputed every time step. It is not restored below the 
mixed layer.

A depth tracer is used to calculate the vertical transport rate over a time interval $\Delta t$. Since the tracer is continuous, it allows us to calculate variance spectra of the vertical transport rate as a function of horizontal wavenumber. The depth tracer is initialized on model day 43.75 (time $t_{0}$ ) with a value that equals its vertical position $z$, such that $\operatorname{Tr}\left(x, y, z, t_{0}\right)=z\left(t_{0}\right)$ in meters. The vertical transport rate $\left(w^{\Delta t}\right)$ over a time interval $\Delta t$ is computed as the difference between the tracer value (in meters) at $t_{0}+\Delta t$ and $t_{0}$ as

$$
w^{\Delta t}=\frac{\operatorname{Tr}\left(x, y, z, t_{0}+\Delta t\right)-\operatorname{Tr}\left(x, y, z, t_{0}\right)}{\Delta t} .
$$

The two-dimensional $(x-y)$ isotropic spectra of $w^{\Delta t}$ is computed using the package pyspec (Rocha, 2015).

\subsection{Results}

\subsubsection{Subduction rate}

The model domain contains a front within the pycnocline, with frontal isopycnals outcropping in the mixed layer, which ranges from $70 \mathrm{~m}$ on the light side of the front to $50 \mathrm{~m}$ on the dense side of the front. During the spin up phase, the front first develops mixed layer submesoscale instabilities. The mixed layer depth (defined as where the local density exceeds the surface density by $0.03 \mathrm{~km} / \mathrm{m}^{3}$ ) shoals to as little as 5 meters along the front, but maintains a depth of 50-70 meter away from the front. By day 20, mesoscale baroclinic instability develops (Figure 4-2). This progression of instabilities is consistent with previous studies on linear unstable modes: a smaller-scale, faster growing mixed layer mode and a larger scale pycnocline mode (Boccaletti et al., 2007; Callies et al., 2016). During the analysis period (model days 44 to 62), instabilities at both the mesoscale and submesoscale are present, but

the regions where the local Rossby number $\left(R o=\frac{\zeta}{f}\right)$ is large, $R o \gtrsim 1$, are mostly localized around the mesoscale front.

Mixed layer water subducts into the pycnocline at a rate of $0.2-0.3 \mathrm{~Sv}$ over the $82,560 \mathrm{~km}^{2}$ domain over course of the analysis period. The subduction rate is computed as the rate 

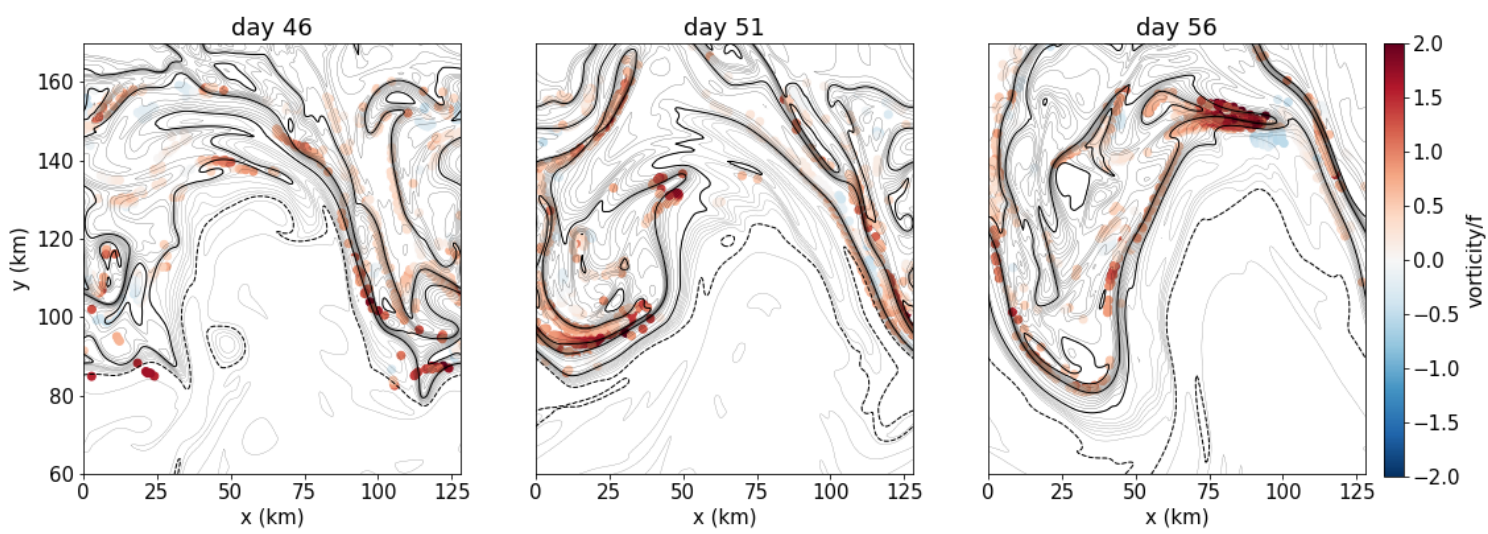

Figure 4-3: Positions $(x-y)$ of particles on the day that they subduct out of the mixed layer. The particles are colored by their relative vorticity, with red referring to cyclonic, and blue to anti-cyclonic vorticity. Density at $5 \mathrm{~m}$ depth is contoured (thin lines CI $=0.02 \mathrm{~kg} / \mathrm{m}^{3}$, thick lines CI $=0.2 \mathrm{~kg} / \mathrm{m}^{3}$ ). Dashed (solid) contours are lighter (denser) than the average surface density. Particles are subducting along the dense side of the fronts where the lateral buoyancy gradient is strongest, and most of the particles have cyclonic relative vorticity. The subduction locations are coherent and elongated in the along-front direction.

of change of the volume of mixed layer water present below the mixed layer. When the subducted volume is normalized by the area of the domain, it amounts to $25 \mathrm{~m}$ of the mixed layer being subducted over a 3-month period (the approximate duration of the winter and early spring conditions simulated here) or $100 \mathrm{~m}$ over a year (if the same subduction rate acts over the year). The subduction rate is diagnosed using the mixed layer tracer and is consistent with past mesoscale and submesoscale resolving simulations. (Gebbie, 2007; Canuto et al., 2018).

The largest values of vertical velocity in this simulation are 30-40 meters/day. Stronger submesoscale vertical velocities have been both observed and modeled (Mahadevan et al., 2010; D'Asaro et al., 2018). The vertical velocity is expected to be stronger when the model includes wind forcing, a parameterization of boundary layer turbulence, deeper mixed layer, stronger mesoscale jet, or smaller value of horizontal eddy diffusivity (Wang, 1993).

\subsubsection{Coherence of subduction}

In the Lagrangian analysis, we consider subduction to occur when a water parcel that was initially in the mixed layer, moves 5 meters below the mixed layer and remains below the mixed layer for the remainder of the 10 day trajectory. We identify the water parcels' sub- 

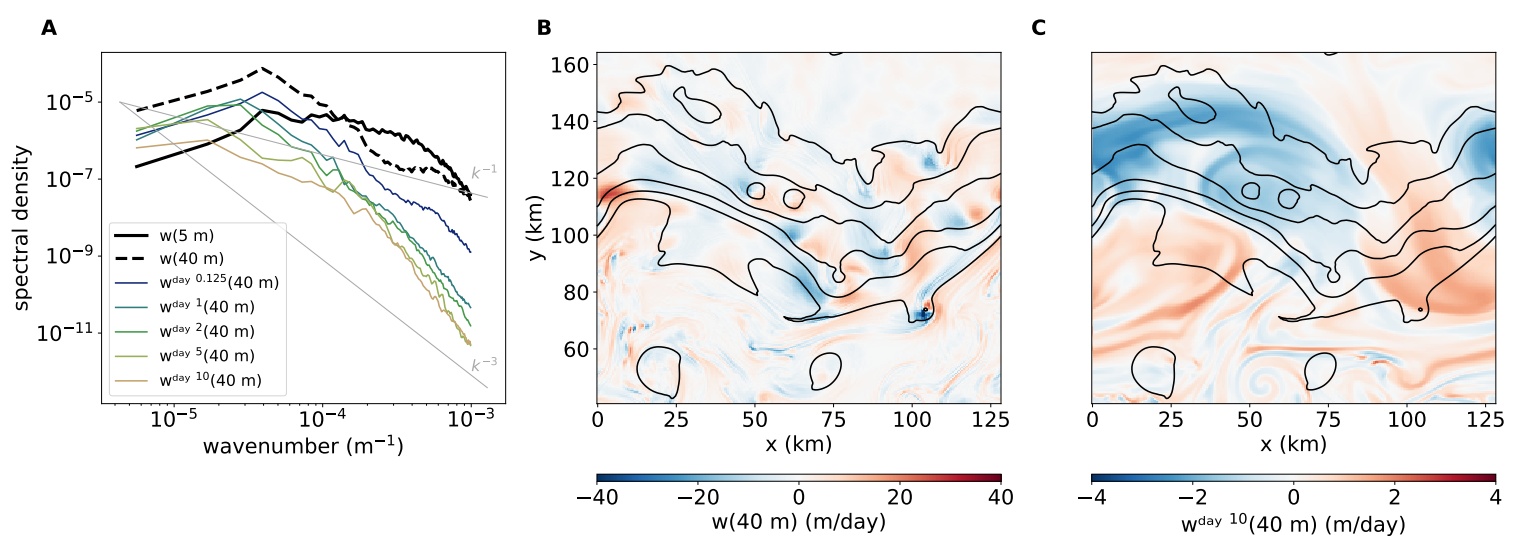

Figure 4-4: (A) Vertical velocity and vertical transport isotropic wavenumber variance spectra. The black lines are the vertical velocity spectrum at (solid) 5 meters depth and (dashed) 40 meters depth. The colored lines show the spectra of the vertical transport at 40 meters depth calculated over a range of time intervals according to (4.5). The grey lines are guides for $k^{-1}$ and $k^{-3}$ slopes. (B) Vertical velocity at 40 meters depth. (C) Vertical transport over 10 days at 40 meters depth.

duction location (horizontal position) and time (model day) (Figure 4-3). This is done by comparing the particles' $z$ location with the local mixed layer depth for each grid cell in the $x-y$ plane and interpolated onto the particle locations at every time step. Of all of the particles seeded evenly across the domain at 5 meters and reseeded daily, $7.7 \%$, or 18,740 , subduct out of the mixed layer. The subduction occurs in localized regions along the front, which leads to coherent features during subduction.

The complex frontal density structure results in a rich variety of features on which water parcels subduct (Figure 4-3). Subduction occurs at the strongest density gradients, which are outcrops of the pycnocline front, and at mixed layer fronts. The subduction locations are almost all located on the dense side (cyclonic side) of the main pycnocline fronts, but intermittently along the front, and in transient features associated with submesoscale filaments and eddies.

Where the water parcels end up after subduction is determined by how subduction interacts with the flow beneath the mixed layer. We use tracer spectra to quantify the transport across different spatial and temporal scales (Figure 4-4). The near-surface (5 m) vertical velocity has variability across a wide range of spatial scales, including small scales, as evidenced by the nearly flat spectrum. Since the vertical velocity goes to zero at the surface, the vertical velocity at 5 meters depth is approximately equal to the surface horizontal divergence 
multiplied by the depth, $\Delta z$,

$$
\left.w \approx \nabla_{h} \cdot \mathbf{u}_{\mathbf{h}}\right|_{z=0}(\Delta z)
$$

At shallow depths, the vertical velocity spectrum $\left\langle w^{2}\right\rangle$ is therefore related to the horizontal velocity spectrum $\left\langle\mathbf{u}_{\mathbf{h}}{ }^{2}\right\rangle$ by

$$
\left\langle w^{2}\right\rangle \sim\left\langle\nabla \cdot \mathbf{u}_{\mathbf{h}}{ }^{2}\right\rangle \sim k^{2}\left\langle\mathbf{u}_{\mathbf{h}}{ }^{2}\right\rangle
$$

At 40 meters depth, which is near the base of the mixed layer, the horizontal velocity has a spectral slope between $k^{-3}$ and $k^{-4}$, but the vertical velocity spectrum is steeper than predicted by (4.7) and not directly related to the local divergence of the horizontal velocity near the surface, because the divergence has variable magnitude and sign between 40 meters and the surface (Figure 4-4A).

The spectrum of the vertical transport defined by (4.5) has lower power than the vertical velocity spectrum because the instantaneous vertical velocity has more extreme values than the time integrated vertical transport. Over time intervals of less than one day, the transport spectrum differs more from the vertical velocity spectrum at high wavenumbers (small spatial

scales). Much of the difference is due to the influence of internal waves on the vertical velocity spectrum, but not the transport spectrum (Balwada et al., 2018). Over time intervals of 5-10 days, the transport spectrum flattens mostly in the smaller wavenumber range. The result is a relatively flat $\left(\sim k^{-1}\right)$ spectrum at spatial scales approximately between 10 and $100 \mathrm{~km}$, expected when interior quasigeostrophic turbulence stirs passive tracers along isopycnal surfaces (Vallis, 2006). At smaller scales, the spectrum is steeper due to a transition in dynamics and likely an increased role for three-dimensional effects, submesoscale stirring, and coherent transport (Spiro Jaeger et al., 2020). Subduction also occurs at these smaller scales.

\subsubsection{Lagrangian description of subduction dynamics}

The time evolution of dynamical properties on the water parcels undergoing subduction from the mixed layer is examined by compositing Lagrangian time histories on a shifted time axis 
with time zero being the day on which the water parcel was subducted (Figure 4-5). The solid line in Figure 4-5 is the median of each property (lateral and vertical buoyancy gradients, relative vorticity, vertical velocity, PV, cyclostrophic acceleration, and frontogenetic terms) and the shaded region is the interquartile range. The properties on half of the subducting particles fall within the interquartilie range, but half fall outside this range. The median of the dynamical properties on the quickly subducting water parcels (water parcels that reach at least $20 \mathrm{~m}$ /day downward vertical velocity) is shown in the dashed line. The time prior to subduction when particles are in the mixed layer is indicated as negative, time zero represents a transition as particles subduct, and the positive time axis shows the period of their evolution after subduction. The evolution of the water parcel properties during subduction sets the properties of the subducted water masses. As expected for downward motion from the mixed layer to the pycnocline, the stratification $\left(N^{2}\right)$ increases (Figure 4$5 \mathrm{~A}$ ) and the vertical velocity increases in magnitude (downwards) (Figure 4-5B). The ratio of the magnitude of the lateral to vertical buoyancy gradient, $M^{2} / N^{2}$, is the isopycnal slope. The isopycnal slope is large in the mixed layer and during subduction, which mostly occurs along steeply sloping isopycnal surfaces $\left(M^{2}\right.$ and $N^{2}$ both increase). The stratification continues to increase as water parcels subduct, but the lateral buoyancy gradient peaks during subduction and then gradually decreases (Figure 4-5A) as subducted water parcels enter a region of higher stratification. This restratification on a Lagrangian trajectory does not necessarily mean that the larger-scale front restratifies. Instead, water parcels are moving along density surfaces into a more stratified region. The mixed layer volume stays relatively constant and does not decrease throughout the analysis period (Figure S2).

The Ertel potential vorticity (PV) is conserved on the water parcels, except during the subduction event, when diabatic processes come into play. In the mixed layer, the PV is near zero due to the low stratification and the negative contribution from vertical shear at strong fronts. During subduction, the total PV on the water parcels increases abruptly (Figure 4-5F). The mixed layer tracer also reveals the same magnitude of increase in PV during subduction in this model (Figure S1), suggesting that the Lagrangian particles stay in the same water parcel during subduction. The subducted water mass still has lower PV 

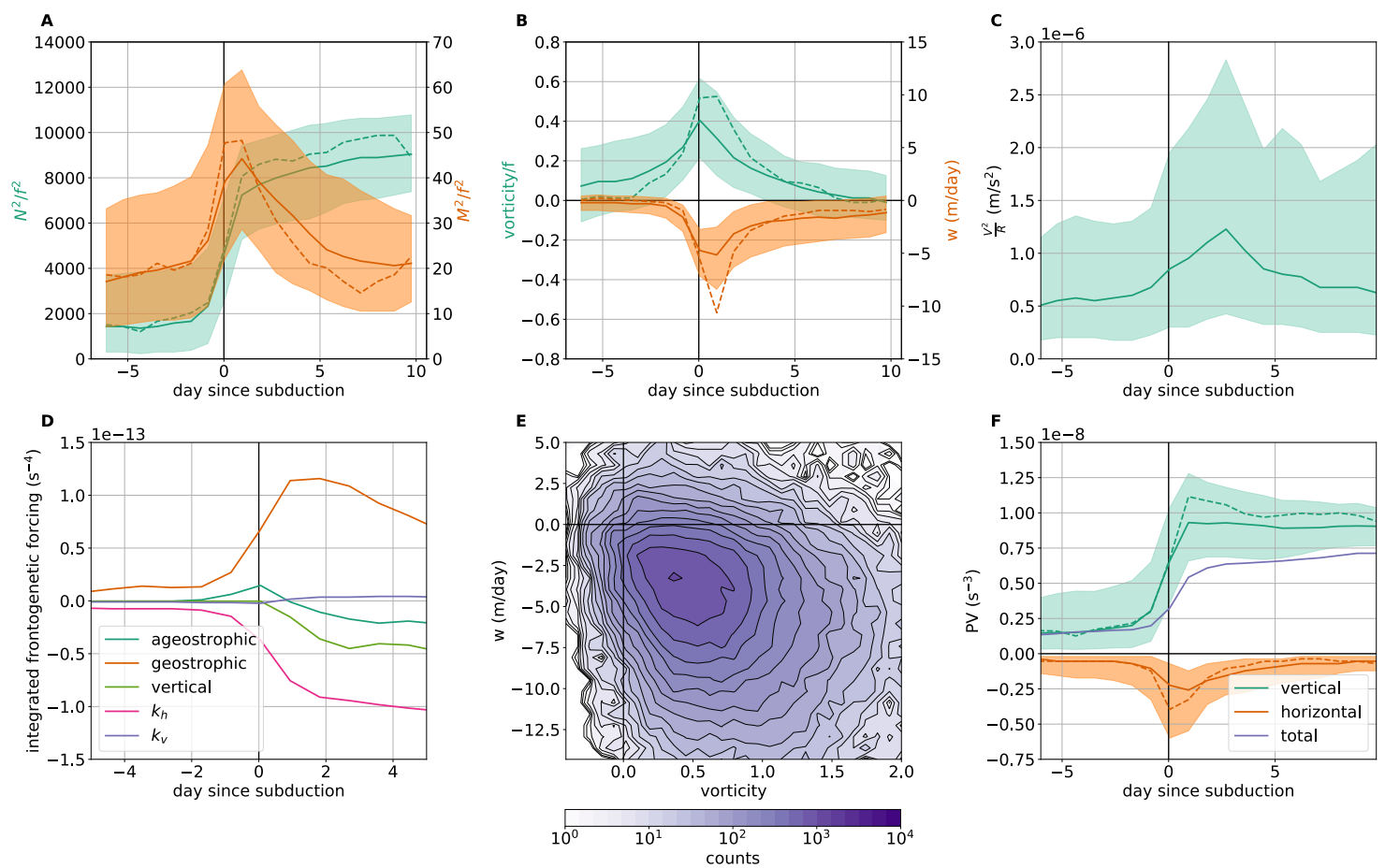

Figure 4-5: Lagrangian evolution of dynamical quantities as water parcels are subducted. All trajectories are composited onto a shifted time axis where time zero is the subduction time of the water parcel (defined as the time when it moves from the mixed layer to 5 meters below the mixed layer depth). The solid line is the median value. The shaded region encloses the first and third quartiles of all subducted particles. Approximately $7 \%$ of all of the particles subduct. The dashed line shows the median of all particles that subduct faster than $20 \mathrm{~m} /$ day. The reference Coriolis frequency used to normalize properties is $f=10^{-4} \mathrm{~s}^{-1}$. (A) Stratification $\left(N^{2} / f^{2}\right)$ (left axis, green) and lateral buoyancy gradient $\left(M^{2} / f^{2}\right)$ (right axis, orange) (B) Relative vorticity normalized by the Coriolis frequency (left axis, green) and vertical velocity (right axis, orange) (C) Non-linear acceleration due to curved particle trajectories. The radius of curvature $(R)$ is calculated using velocities and accelerations averaged over 15 hours on particle trajectories. (D) Various contributions to frontogenesis, represented by the terms on the right hand side of Equation (4.2) integrated in time along particle trajectories. The lines show the median values of all subducted particles on a shifted time axis. Positive values are frontogenetic and negative values are frontolytic. The $\mathbf{Q}$-vector is split into geostrophic and ageostrophic components. The third term is frontogenesis by the vertical velocity ("vertical"). The fourth term is horizontal diffusion $\left(k_{h}\right)$. The fifth term is vertical diffusion $\left(k_{v}\right)$. (E) Two dimensional histogram showing the relationship between the vertical component of relative vorticity (normalized by $f$ ) and the vertical velocity on particles during the 20 hours prior to subduction. (F) Vertical and horizontal contributions to the PV. The purple line is the median of the total PV. 
on average than the interior water mass. The relevance of diabatic processes in subduction suggests that improved representation of the frontal arrest process (Bodner et al., 2020) and boundary layer turbulence (Gula et al., 2014) could have large implications for exchange between the mixed layer and interior.

The PV can be split into a vertical (vortical) contribution $\left(q_{v}\right)$ and a horizontal (baro-

clinic) contribution $\left(q_{h}\right)$. The vertical contribution $q_{v}$ remains elevated after the water parcels have subducted. The magnitude of $q_{h}$, which is negative, increases transiently as the water parcels subduct (Holmes et al., 2014). The increase in the magnitude of $q_{h}$ and decrease in the vertical component of relative vorticity $(\zeta)$ do not counter the increase in $q_{v}$ that arises from the increase in stratification, $N^{2}$.

\section{Frontogenesis}

The intensification of the lateral buoyancy gradient all particle trajectories prior to and during subduction demonstrates that frontogenesis plays an important role in subduction (Figure 4-5A). On average, frontogenesis occurs here due to straining by the geostrophic velocities. The ageostrophic strain also contributes to average frontogenesis during subduction (Figure 4-5D; Equation 4.2). After subduction, frontolysis is mainly due to the ageostrophic overturning circulation and horizontal diffusion (Figure 4-5D). In situations with stronger vertical velocity, the ageostrophic frontogenesis terms could be more important (Barkan et al., 2019). We describe a few cases where ageostrophic frontogenesis dominates in submesoscale features (Section 4.5.4).

Concurrent with frontogenesis, the vertical component of the relative vorticity, $\zeta$, increases rapidly on water parcels as they approach the subduction location, after which their $\zeta$ decreases (Figure 4-5B). Nearly all of the water parcels have cyclonic vorticity when they initially subduct out of the mixed layer (Figure 4-3) and about half develop anticyclonic vorticity by the end of the trajectory (Figure 4-5B). The third quartile of $\zeta$ on subducting water parcels reaches $\sim 0.6 f$. The average rate of subduction on trajectories is fairly slow, at 1-4 meters per day. However, the vertical velocity, $w$, during and immediately after subduction can be large $(20-30 \mathrm{~m} /$ day $)$. At the time of subduction, $\zeta$ and $w$ are negatively correlated, but the largest values of $w$ have a significant vorticity range (Figure 4-5E). On 
average, $\zeta$ on water parcels peaks ahead of the downward $w$ (Figure 4-5B) due to the relationships between vorticity and vertical velocity (Equation 4.4). At the sea surface, $w$ approaches zero, but $\zeta$ often reaches a maximum; vortex stretching is due to the vertical shear of the vertical velocity.

\section{Curvature of trajectories}

The large values of cyclonic vorticity on the dense side of the front contribute to strengthening the PV gradient and make the front susceptible to baroclinic and barotropic instabilities that manifest as frontal waves. The increase in cyclonic vorticity on the water parcels is due to two factors: (i) shear on the edge of the jet, which is a $2 \mathrm{D}$ mechanism (cross-front and in the vertical) and is described by the semi-geostrophic Omega equation, and (ii) curvature of the trajectories, a 3D process that includes along-front variability and impacts the

dynamics through a cyclostrophic acceleration, $\frac{V^{2}}{R}$ where $V$ is the velocity magnitude and $R=\frac{\left(u^{2}+v^{2}\right)^{3 / 2}}{u v_{t}-v u_{t}}$ is the radius of curvature. If the cyclostrophic term is the same order of magnitude as the Coriolis term, the balanced velocity will be in gradient wind balance rather than geostrophic balance. The cyclostrophic term is larger when the flow is more non-linear. On the particle trajectories, the cyclostrophic acceleration increases prior to subduction, peaks 2-3 days after subduction on average, and then decreases (Figure 4-5C). The water parcels have cyclonic vorticity during subduction, so the cyclostrophic term decelerates the velocity relative to the geostrophic velocity, resulting in a convergence and downwelling along the particle trajectories. In this way, the spiralling of water parcels helps to maintain downward transport. Both the cyclostrophic acceleration (McWilliams et al., 2019) and the conversion from the baroclinic to the vortical contributions of the PV (Thomas, 2008) reveal the importance of along-front curvature in driving subduction.

\subsubsection{Subduction by submesoscale features}

Examining particle trajectories on isopycnal surfaces reveals some of the submesoscale processes that enhance subduction. Restratification and along-isopycnal stirring may work in tandem to promote subduction. As the base of the mixed layer restratifies (Fox-Kemper 


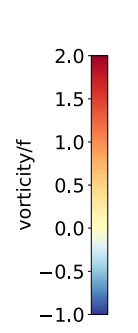

A1
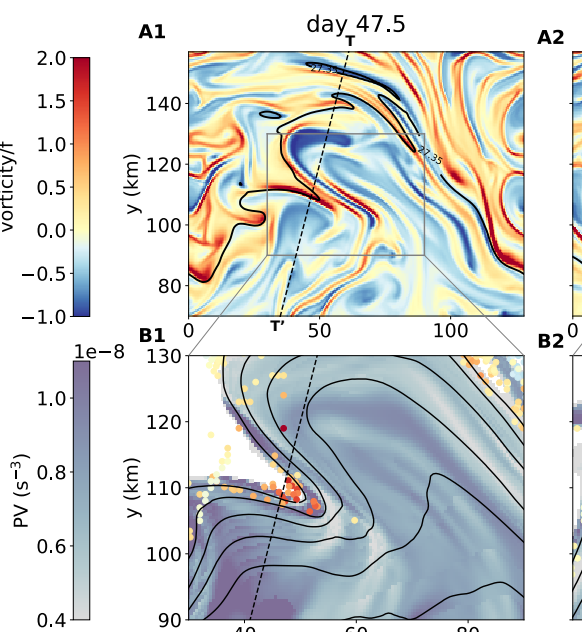

है
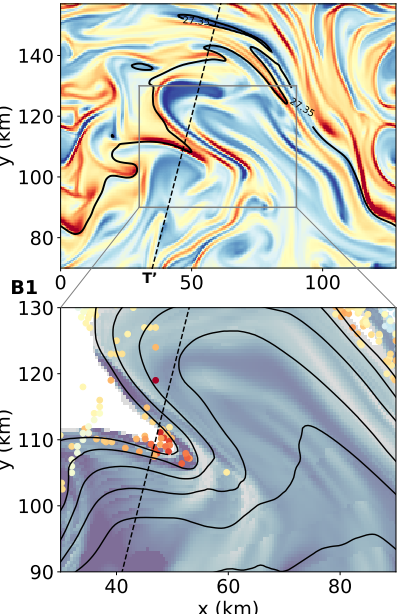

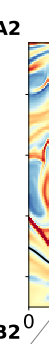

day 48.5
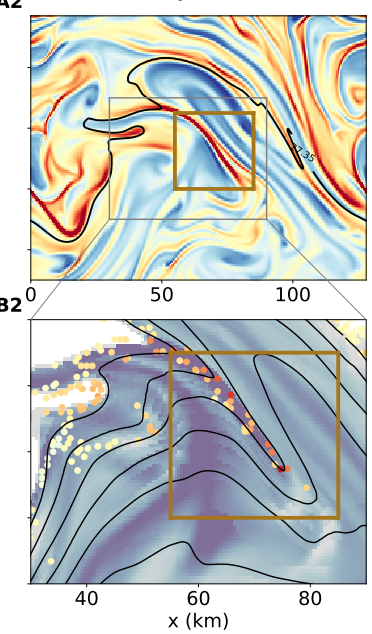

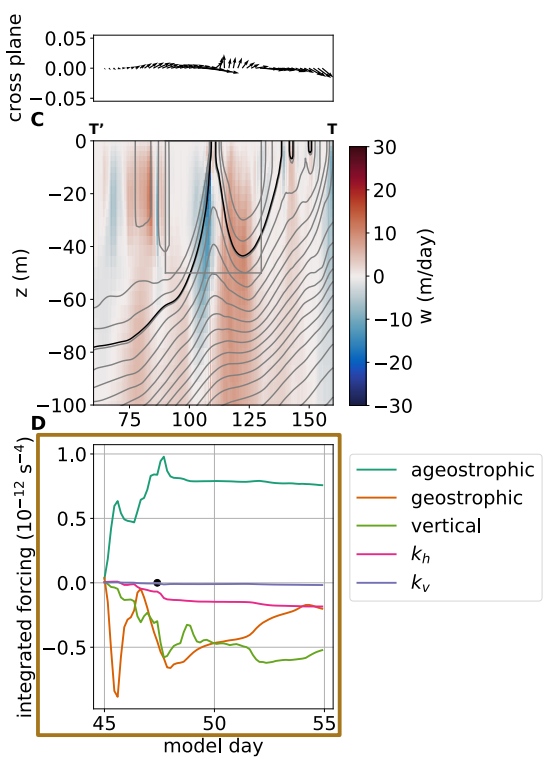

Figure 4-6: A submesocale filament as a pathway for subduction from the mixed layer. The evolution of a cyclonic filament is shown in panels A, B. (A) Surface relative vorticity normalized by the Coriolis frequency on days 47.5 (1) and 48.5 (2). The contour is $\sigma=27.35$. (B) $\mathrm{PV}$ on the $\sigma=27.35$ surface. The black contours are isopycnal height at 10 meter intervals. All particles shown have density within $0.01 \mathrm{~kg} / \mathrm{m}^{3}$ of the isopycnal surface and subduct below the mixed layer during their trajectory. The particles are colored by their relative vorticity. (C) Lower panel: Cross section of the vertical velocity in meters/day on day 47.5 along the black dashed line $\mathrm{T}^{\prime}-\mathrm{T}$ in panel $\mathrm{A}(1)$. Contours are isopycnals. The black contours $\sigma=27.8$. Upper panel: surface horizontal velocity in the along plane direction (x-axis) and cross plane direction. Downwards in the cross plane direction is out of the page (positive $\mathrm{x}$ direction). (D) Mean time-integrated frontogenetic forcing on the particles within the orange box in panels A,B. Positive slopes are frontogenetic and negative slopes are frontolytic. Contributions to frontogenesis are split into the terms on the right hand side of Equation (4.2). The black dot on the $\mathrm{x}$-axis indicates the time of subduction. 

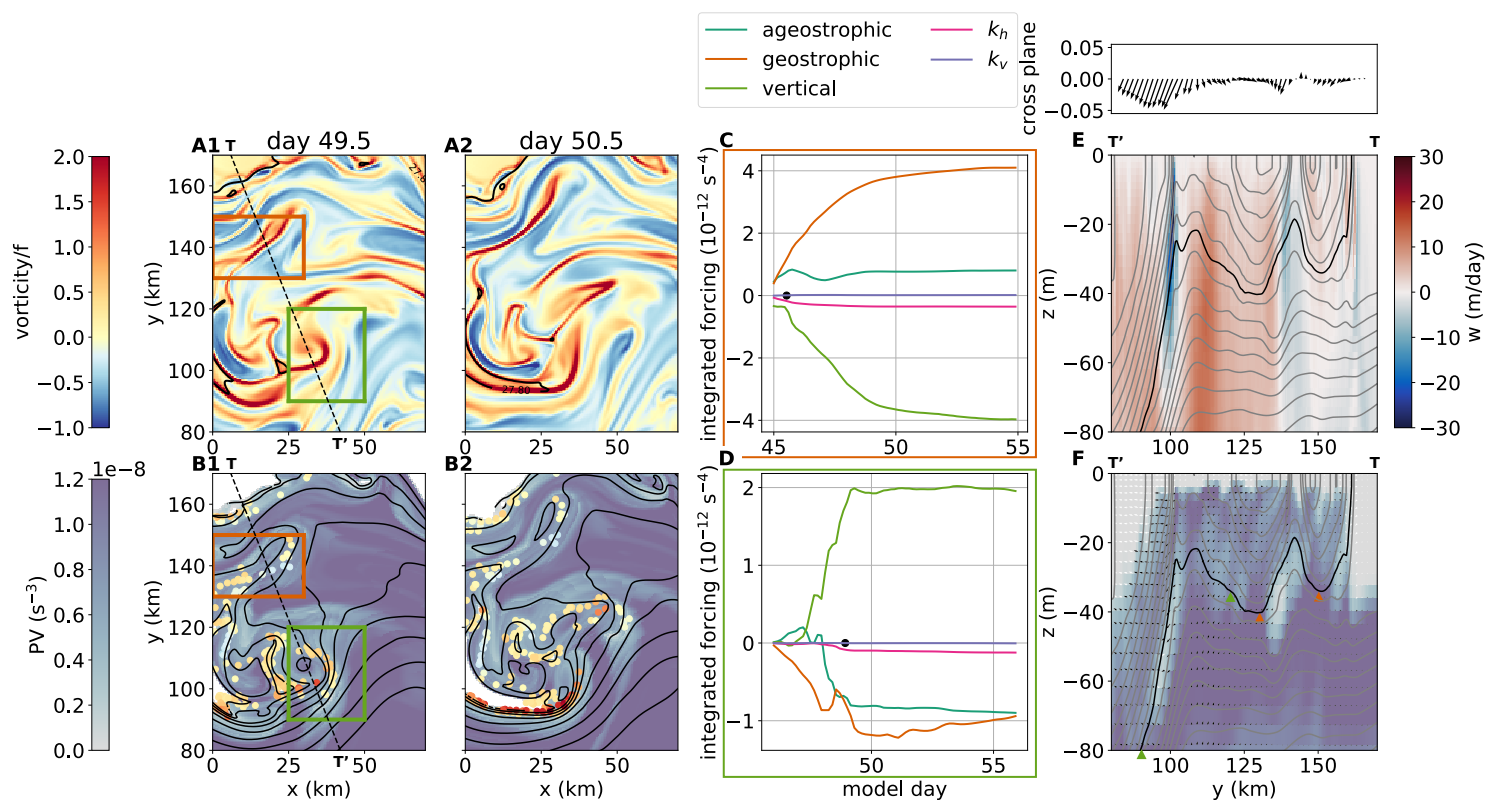

Figure 4-7: Two specific pathways for subduction from the mixed layer form an intrapycnocline eddy (orange box) and cut-off cyclone (green box). (A) Surface relative vorticity normalized by the Coriolis frequency on days 49.5 (1) and 50.5 (2). The contour is $\sigma=$ 27.8. (B) PV on the $\sigma=27.8$ surface. The black contours are isopycnal height at 10 meter intervals. All particles shown have density within $0.01 \mathrm{~kg} / \mathrm{m}^{3}$ of the isopycnal surface and subduct below the mixed layer during their trajectory. The particles are colored with their relative vorticity. (C,D) Contributions to frontogenesis split into the terms on the right hand side of Equation (4.2). The forcing due to each term is integrated in time along each subducting particle trajectory. (C) Mean time-integrated frontogenetic forcing on the particles within the orange box in panels A,B (D) Mean time-integrated frontogenetic forcing on the particles within the green box in panels A,B. Positive slopes are frontogenetic and negative slopes are frontolytic. The black dot on the x-axis indicates the time of subduction. (E, F) Cross sections on day 49.5 at the black dashed line on panels A,B. Contours are isopycnals. The black contour is $\sigma=27.8$. (E) Upper panel: surface horizontal velocity in the along plane direction (x-axis) and cross plane direction. Downwards in the cross plane direction is out of the page (positive $\mathrm{x}$ direction). Lower panel: Vertical velocity in meters per day. (F) PV with velocity vectors. The low PV anomaly between the orange markers is an intrapycnocline eddy formed by subduction. The green and orange triangles show the y locations of the green and orange boxes in panels A1,B1. 
et al., 2008), water parcels are trapped in a transition layer with reduced turbulence (Shcherbina and D'Asaro, 2020; Taylor et al., 2020) and are more likely to subduct along isopycnals into the pycnocline.

While water parcels move upward and downward in the pycnocline as they transit through the crest and trough of a mesoscale meander (Bower et al., 1985; Bower, 1991; Samelson, 1992), the along-front structure facilitates the growth of submesoscale features that contribute to subduction from the mixed layer. Subduction is analyzed using equation (4.2) in three submesoscale features that are found to differ in their frontogenesis mechanisms. The processes described here differ from subduction in other physical situations that are dominated by mixed layer eddies (Boccaletti et al., 2007; Omand et al., 2015; Shcherbina et al., 2015; McWilliams, 2016) or symmetric instability (Thomas et al., 2013; Erickson and Thompson, 2018). Here, subduction from the surface mixed layer to the interior occurs through the combination of mesoscale and submesoscale processes.

\section{Submesoscale filaments}

As the vorticity rapidly increases at the mesoscale front due to geostrophic frontogenesis, the front develops wave-like features at the sharpest density gradient (Figure 4-6A) due to horizontal shear and baroclinicity. As the wavy instabilities grow, they further intensify due to ageostrophic secondary circulations, and wrap into submesoscale vortices or elongate into filaments. Submesoscale dense filaments are known to rapidly intensify through a dual frontogenetic process called filamentogenesis (McWilliams et al., 2009; Gula et al., 2014). A filament generated by geostrophic and ageostrophic frontogenesis has large cyclonic vorticity (Figure 4-6A) and downwelling vertical velocity at its center (Figure 4-6C). Water parcels subduct out of the mixed layer along a sloping isopycnal ( Figure 4-6B) during the development of the filament. There is upwelling on either side of the subduction region that brings up high PV water that is mixed into the subduction region both adibatically along the isopycnal surface (Figure 4-6B) and diabatically (not shown) resulting in a subducting water mass that has high PV relative to surrounding water on the same isopycnal surface (Figure 4-6B). After subduction, the water parcels are transported more than $80 \mathrm{~km}$ laterally across the domain and continue to subduct as the cyclonic filament relaxes. This example 
reveals a pathway to subduct anomalously high PV attained through diapycnal mixing with surrounding water.

Here, (Figure 4-6D) the filament intensifies by both geostrophic and ageostrophic frontogenesis (the rate of change of the integrated frontogenetic forcing is positive) (Barkan et al., 2019) and decays due to geostrophic frontolysis, horizontal diffusion and vertical transport. Other model studies with 500 m grid resolution, but smaller horizontal diffusivity and boundary layer turbulence parameterized by KPP, have found that filamentogenesis is halted by secondary instabilities of the filament rather than by diffusion (Gula et al., 2014; Barkan et al., 2019), in contrast to lower resolution models $(1.5 \mathrm{~km})$.

Previous observational and modeling studies have shown that cold (cyclonic) submesoscale filaments in the Gulf Stream and Antarctic Circumpolar Current can contribute to exchange between the surface and pycnocline (Gula et al., 2014; Klymak et al., 2016; Taylor et al., 2018).

\section{Intrapycnocline eddies}

Quasigeostrophic theory predicts that subduction occurs due to geostrophic frontogenesis, which generates ageostrophic horizontal and vertical velocities. As low PV surface mixed layer water crosses the front it subducts along an isopycnal surface while conserving quasigeostrophic PV, $q=\frac{f+\zeta}{H}$, where $H$ is the layer thickness. If the mixed layer is thicker than isopycnal layers in the thermocline, the subducted water mass becomes an anticyclonic intrapycnocline eddy as it is compressed during subduction (Spall, 1995). The length scale of the intrapycnocline eddy is expected to be the internal deformation radius. The radius of deformation of the mixed layer is $\frac{N_{0} D}{f} \approx 3 \mathrm{~km}$ where $N_{0}^{2}=10^{-4} \mathrm{~s}^{-1}$ is the reference stratification, $D=30 \mathrm{~m}$ is the mixed layer depth, and $f=10^{-4} \mathrm{~s}^{-1}$.

An intrapycnocline eddy can be identified in a cross section as a low PV anomaly, which in Figure 4-7F is shown bounded by the orange triangles and has a radius slightly smaller than $3 \mathrm{~km}$. The formation of a low PV intrapycnocline eddy on the $\sigma=27.8$ isopycnal surface is highlighted in the brown box in Figure 4-7B. The water parcels that become this intrapycnocline eddy are subducted from a region of large cyclonic vorticity (Figure 4-7A,B: orange box) due to geostrophic frontogenesis with some contribution from ageostrophic fron- 
togenesis (Figure 4-7C, equation (4.2)). The water parcels subduct on a dense (cyclonic) filament that outcrops at the center of the front. The intrapycnocline eddy that forms during subduction moves towards the dense side of the front and is elongated as it subducts into a region where the vertical branch of the ageostrophic circulation is frontolytic. The water parcels, which initially have large values of cyclonic vorticity, develop weakly anticyclonic vorticity within one day (vorticity is in colors on the water parcels in Figure 4-7B). The intrapycnocline eddy has a cyclonic surface expression (Figure 4-7A).

Intrapycnocline eddies have been observed near fronts (Archer et al., 2020) and anticyclonic submesoscale vortices, which were likely generated at fronts have been observed more generally. These eddies trap material in the interior and are potentially important for transporting heat, salt, and biological communities (Frenger et al., 2017). In this study, we observe that the subducted water mass fills the center of the subducted feature, supporting non-local coherent material transport.

\section{Cut-off cyclones}

The mixed layer base, not just the sea surface, is involved in the formation of a submesoscale cyclone (Figure 4-7A,B: green box) as differential vertical motion intensifies the front by tilting the stratification at the mixed layer base onto the horizontal (Figure 4-7D). The cyclonic vorticity is largest on the edges rather than the center of this eddy (Figure 4-7A: green box). The core of the submesoscale cyclone has high PV, rather than the low PV that would be expected from an intrapycnocline eddy. Low PV mixed layer water parcels leave the mixed layer around the edges of the cyclone. While subduction is 3D in the surface layer, once water parcels leave the mixed layer, they are transported along isopycnal surfaces and the subduction has a more $2 \mathrm{D}$ character resulting in a low PV water mass at depth that has weakly cyclonic vorticity (Figure 4-7E, near $100 \mathrm{~km}$ ).

The dynamics and kinematics of the modeled cyclone reflect the formation process of cutoff cyclones, or cutoff lows, in atmospheric dynamics (Rotunno et al., 1994). Cutoff lows are known to be important for stratosphere-troposphere exchange (Holton et al., 1995; Fuenzalida et al., 2005). Related dynamics in the ocean may naturally be expected to be relevant for exchange between the mixed layer and thermocline. A similar feature to the modeled 
cyclone was observed in a section across the western Alborán Gyre (Figure 4-1B,C, near $50 \mathrm{~km}$ ). Frontal waves and eddies that are qualitatively similar to this modeled feature and the feature on the Alborán Gyre observations have been observed previously, mostly from satellites and photographs from space shuttles, and have been attributed to both shear instability and baroclinic instability (Munk et al., 2000; Yin and Huang, 2016; Klymak et al., 2016). In both cases, the waves are observed to become unstable in 2-3 days and have wavelengths of $20-30 \mathrm{~km}$, consistent with the modeled features.

\subsection{Discussion}

This process study reveals the challenge of separating submesoscale and mesoscale processes, which may feedback on each other. The mesoscale front provides sloping surfaces along which water parcels can be exported into the pycnocline. Subduction patterns are largely driven by along-front variability of the mesoscale meander, which generates ageostrophic secondary circulations through frontogenesis and is shaped by those same ageostrophic secondary circulations (McWilliams et al., 2019). A variety of submesoscale features, either cyclonic or anti-cyclonic, support subduction that occurs along high or low PV pathways. Water masses that are subducted from the surface mixed layer into the pycnocline can have anomalously low stratification and PV compared to surrounding waters and thereby develop anticyclonic relative vorticity (Pollard and Regier, 1992). However, subduction of water mass anomalies and biogeochemical tracers can occur along sloping density surfaces even without generating a volume anomaly and therefore could also occur along high PV pathways (Freilich and Mahadevan, 2019). High PV water parcels are upwelled and subducted along isopycnal surfaces in regions with vertical circulation that is so rapid that surface forcing would not have time to remove PV at the surface. At the submesoscale, the cyclonic curvature of water parcel trajectories that encounter frontal waves would alter the thermal wind balance and modify the ageostrophic overturning. The presence of the mesoscale front may in some situations enhance submesoscale instability (Rotunno et al., 1994), but mesoscale fronts may also stabilize the flow through strain or barotropic shear (Gula et al., 2016; Taylor et al., 2018; Stamper et al., 2018). While subduction locations may be short lived and the residence 
time in subduction locations very short, along-front variability in both horizontal and vertical velocity patterns breaks the periodicity of the meander (Bower, 1991; Samelson, 1992) resulting in longer-term subduction.

These results have implications for the parameterization of subduction processes, since they are found to be are both local and non-local. If the subduction were completely incoherent it could be appropriately modeled as a diffusive process. If subduction took place through a steady overturning process, it would either be completely reversible or only associated with restratification. We find that the exchange across the mixed layer base occurs at $\mathcal{O}(10 \mathrm{~km})$ scales and is important for supplying oxygen to the pycnocline and nutrients to the mixed layer. The spatial scale of the subduction affects the upper ocean thermohaline structure (Cole and Rudnick, 2012; Spiro Jaeger et al., 2020) and biogeochemical tracer distributions (Erickson and Thompson, 2018). Improved process level understanding of exchange across the mixed layer base could lead to improved estimates of upper ocean productivity and pycnocline ventilation (Jin et al., 2007; Llanillo et al., 2018).

Eddy fluxes at the meso- and submeso-scale are increasingly recognized as important for the transport of water masses and biogeochemical tracers from the surface to the interior (Omand et al., 2015; Balwada et al., 2018; Canuto et al., 2018; Resplandy et al., 2019). Submesoscale processes will be most important during times of the year and in locations with deep mixed layers, namely the winter and early spring but may only affect oxygen and carbon transport on annual timescales if the water parcels are transported below the deepest wintertime mixed layer. (Palevsky and Nicholson, 2018) The seasonal restratification of the mixed layer is an important process for subduction of carbon and oxygen into the pycnocline, but the stratified waters may be re-entrained into the mixed layer the following winter Transport of water masses across the mixed layer base without restratifying the mixed layer is a mechanism for transport below the deepest mixed layer on interannual timescales. 


\subsection{Conclusions}

Subduction processes from the mixed layer into the interior are organized by, but not entirely controlled by, the mesoscale meandering jet. By analyzing the trajectories of water parcels we find that submesoscale features generated through baroclinic instability of the mesoscale front open up pathways into the interior. As water parcels subduct, they experience increased horizontal and vertical buoyancy gradients and a peak in the relative vorticity. While geostrophic frontogenesis drives the majority of subduction in this study, we also outline the importance of ageostrophic and three-dimensional processes for subduction and illustrate these subduction processes with specific examples. Even in locations of net mesoscale upwelling, submesoscale dynamics subduct water from the mixed layer. Subduction from the mixed layer is mostly localized at the strong mesoscale front, but occurs episodically along that front due to frontogenetic processes with high spatial and temporal variability. This variability reduces the likelihood of re-entrainment of water parcels into the mixed layer and determines the spatial scale of exchange between the surface and interior. The subduction is coherent on spatial scales of order $1-10 \mathrm{~km}$, as was observed in the Western Mediterranean. The dynamical subduction by the submesoscale has a similar subduction rate to larger scale processes, but is not resolved in global models. 


\title{
Chapter 5
}

\section{Advective carbon export in an}

\section{oligotrophic gyre by coupled}

\section{mesoscale-submesoscale dynamics}

\begin{abstract}
The sinking flux of organic carbon in subtropical gyres is not sufficient to supply observed heterotrophic production in the ocean interior. Physically-driven transport of carbon from the ocean surface to the interior is an additional possible carbon source. Here, we show that coupled mesoscale-submesoscale dynamics at ocean fronts and eddies are a source of particulate organic carbon (POC) to the mesopelagic ocean. Using surveys in the Western Mediterannean Sea we demonstrate that three-dimensional intrusions with elevated POC, oxygen, chlorophyll fluorescence, and anomalous water mass properties are a pathway for POC transport. By analyzing the community composition with flow cytometry and $16 \mathrm{~S}$ rRNA gene V1-V2 sequencing, we demonstrate that heterotrophic bacteria contribute at least half of the elevated POC. The intrusions transport microbial communities from the surface to the interior, resulting in alterations in the community composition at depth. The biological community serves as a tracer of export indicating the origin of the subducted biomass. The communities are transformed within the intrusions as the POC is remineralized on timescales of days.
\end{abstract}

\section{$5.1 \quad$ Introduction}

Subtropical gyres are one of the largest ecosystems on earth, covering $40 \%$ of the ocean surface area (Polovina et al., 2008). Surface waters in subtropical gyres, where there is enough light for photosynthesis, typically are oligotrophic meaning that nutrients are at very low 
concentrations. In these regions, vertical nutrient supply due to eddies and fronts plays an important role in sustaining production (McGillicuddy et al., 1998b; Freilich and Mahadevan, 2019). Due to the exponential decay of light with depth, even small perturbations to the depth of the nutricline in the vertical can result in phytoplankton blooms. Over an annual cycle, new production that occurs from the fresh supply of inorganic nutrients is balanced by export. The traditional view of carbon export is a vertical flux due to sinking organic material (Siegel et al., 2016; Palevsky and Doney, 2018). However, current estimates of sinking fluxes of organic matter are not sufficient to account for the observed levels of heterotrophic production in the mesopelagic or the observed levels of annual net community production (Palter et al., 2005; Alonso-González et al., 2009; Baltar et al., 2012; Emerson, 2014; Giering et al., 2014; Roshan and DeVries, 2017; Chen and McKinley, 2019).

Accounting for physical advective transport could help close surface biogeochemical budgets. Advective transport of carbon from the surface to the interior, a mostly adiabatic downward supply of carbon that opposes upward nutrient fluxes, can enhance the export of small, slowly sinking phytoplankton cells and of dissolved organic carbon (DOC). While sinking flux is dominated by larger particulate matter, small phytoplankton are a dominant part of the phytoplankton biome in oligotrophic regions. As the ocean becomes warmer and more stratified with climate change, small phytoplankton are expected to constitute a larger fraction of the ocean primary production (Behrenfeld et al., 2006; Flombaum et al., 2013). Even in the nutrient-replete subpolar North Atlantic where it was previously thought that blooms are dominated by diatoms, small phytoplankton have been observed to dominate the bloom biomass (Bolaños et al., 2020). Previous work suggested that small photosynthetic cells are present in the mesopelagic in subtropical gyres, supporting our hypothesis that they contribute to carbon export (Richardson and Jackson, 2007; Lomas and Moran, 2011; Sohrin et al., 2011; Guidi et al., 2016). This previous work has proposed biological mechanisms for the transport of small cells to depth such as aggregation and sinking or consumption by higher trophic levels.

Advective biogeochemical fluxes in oligotrophic gyres are likely driven by eddy processes at the mesoscale and submesoscale. In the high latitudes, mixed layer eddy-driven subduction and restratification have been observed to export carbon from the surface ocean into the 
interior (Omand et al., 2015; Dall'Olmo et al., 2016; Balwada et al., 2018; Canuto et al., 2018; Llort et al., 2018; Resplandy et al., 2019). Subducted mixed layer carbon can persist for months as coherent features that are detectable in vertical profiles (Llort et al., 2018; Johnson and Omand, 2021). However, mixed layer eddy-driven export is expected to be weak in mid-latitude oligotrophic subtropical regions because the mixed layers are shallow (Omand et al., 2015; Johnson et al., 2016). Previous observational and modeling work suggests that mesoscale eddies and frontal meanders could play a role in subduction of phytoplankton in subtropical gyres and in coastal upwelling regions (Tintore et al., 1988; Washburn et al., 1991; Guidi et al., 2012; Stukel et al., 2017; Resplandy et al., 2019; Ruiz et al., 2019). Eddy exchange between the surface and the interior occurs at fronts, the meeting between two water masses, because the sloping density surfaces provide pathways for vertical motion. Here we describe and quantify the impact of coupled mesoscale-submesoscale frontal dynamics on carbon export and community composition in an oligotrophic gyre using field observations in the Mediterranean Sea.

\subsection{Hydrographic and biogeochemical setting}

We sampled the Alborán Sea during three research cruises which took place July 17-24, 2017 on the B/O Socib (IRENE), May 27-June 2, 2018 on the NRV Alliance (Calypso 2018; CLP18), and March 21-April 12, 2019 on the R/V Pourquoi Pas? (Calypso 2019; CLP19) (Table 5.1). There are three characteristic water masses in the Western Mediterranean, which are defined by their temperature-salinity properties (Figure 5-2). The "Atlantic Water" is the most recent inflow from the Atlantic Ocean and is the freshest and coolest water mass. The older - meaning more time in the Mediterranean Sea - "Mediterranean Water" is warm and salty. The "Modified Atlantic Water" sits between these two water masses in

temperature-salinity space. Fronts form at the confluence of these watermasses. The fronts have steeply sloping isopycnals and a large contrast in surface density where the isopycnals outcrop. This density structure generates an along-front velocity of $0.5-1 \mathrm{~m} / \mathrm{s}$. The fronts form the boundaries of gyres and eddies. The Western Alborán Gyre (WAG) is a persistent anticyclonic gyre formed by the Atlantic inflow. The frontal structure is more variable to 


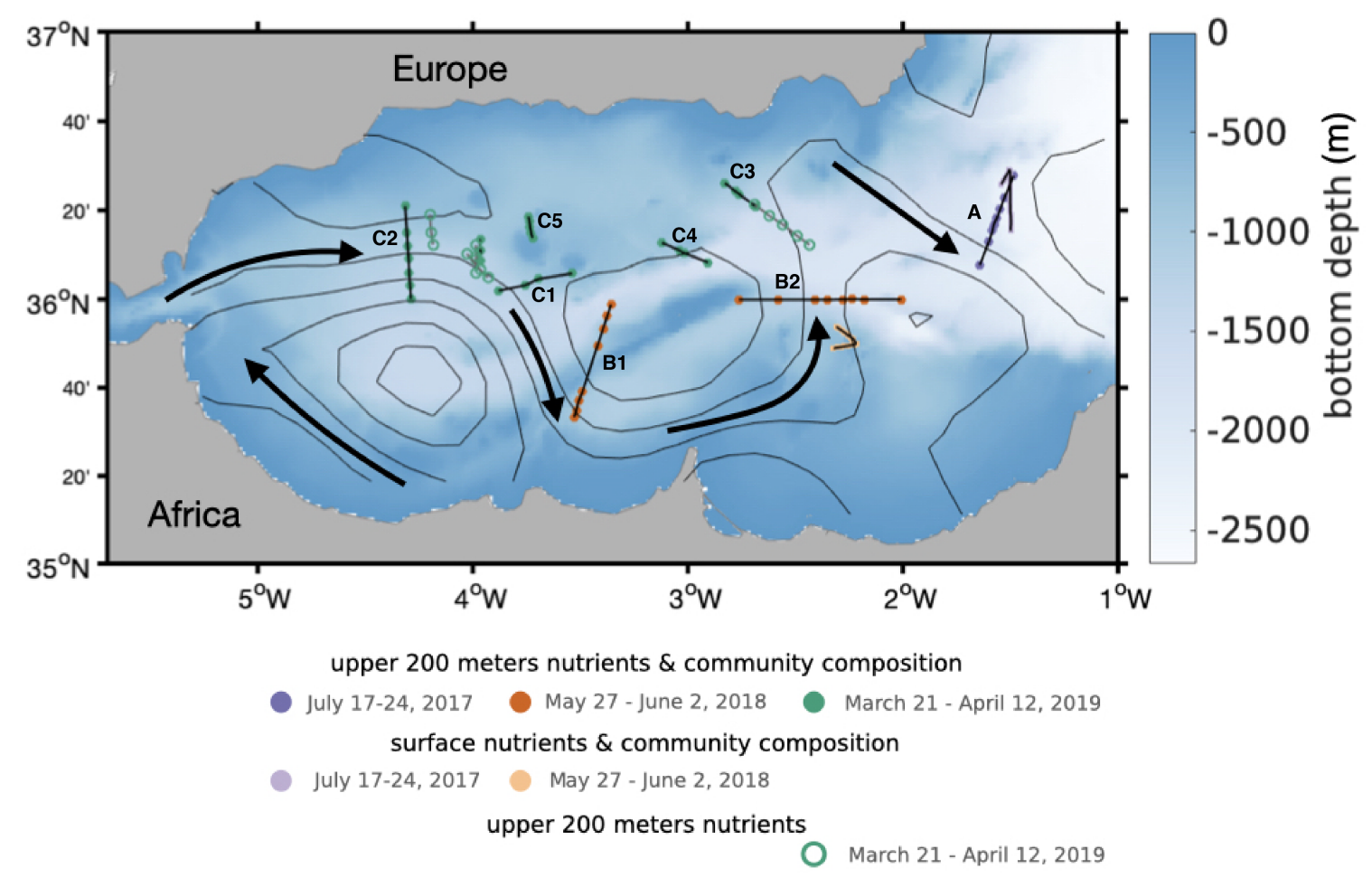

Figure 5-1: Sampling locations. The contours show the sea surface height in May 2018. The arrows are provided to aid conceptual interpretation and show the direction of the currents. Points show the locations of CTD casts. Black lines connect CTD transects that took place within a short time period.

the east of the WAG with a second gyre, the Eastern Alborán Gyre (EAG), which is present much of the time, but not always (Renault et al., 2012). The EAG was present during our cruise in 2017 and 2018 but not in 2019. However, in 2019 there was an anticyclonic eddy in the northern part of the basin at the confluence of the Modified Atlantic Water and Mediterranean Water.

Primary production in the Alborán Sea is largely driven by upwelling at fronts and coastal upwelling (Claustre et al., 1994; Estrada, 1996; Oguz et al., 2014; Olita et al., 2017; Ruiz et al., 2019). The Atlantic water has more nutrients than the older Mediterranean water and creates a shallower nutricline. Mesoscale features in the Western Mediterranean have vertical motion with rates of $20 \mathrm{~m}$ /day (Allen et al., 2001), which can stimulate productivity and shifts in phytoplankton community composition (Morán et al., 2001; Rodriguez et al., 2001) and subduct water masses and carbon from the surface to the interior (Tintore et al., 1988; Fielding et al., 2001; Ruiz et al., 2019; Brett et al., 2020). As in other oligotrophic 

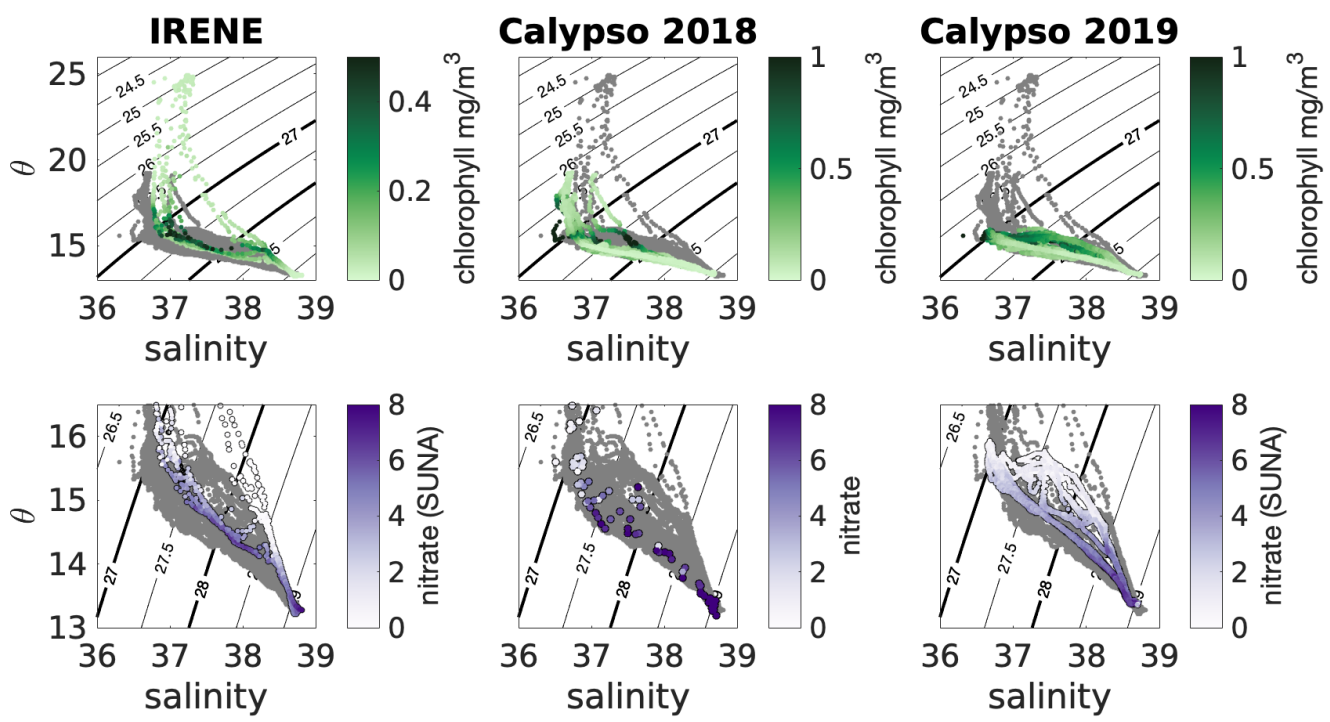

Figure 5-2: Temperature-salinity diagrams with biogeochemical data. The grey points show temperature-salinity from the ship-board CTD from all cruises and are the same on every panel. Chlorophyll (top) and nitrate (bottom) measurements are shown from each research cruise (columns). Chlorophyll measurements are from the ship-board CTD fluorometer calibrated with in situ samples. Nitrate measurements are from the SUNA attached to the shipboard CTD, calibrated with in situ samples. Nitrate measurements from Calypso 2018 are only in situ samples. The temperature axis limits are different for each row.

regions, throughout much of the year there is a deep chlorophyll maximum in the Alborán Sea, which is also a biomass maximum (Olita et al., 2017; Marañón et al., 2021). The deep biomass maximum is shallower in the Atlantic waters than in the Mediterranean waters and is positioned at the nutricline (Lohrenz et al., 1988).

During the two summertime cruises (IRENE and CLP18), the surface mixed layer was shallow $(5-25 \mathrm{~m})$, stratified and lighter and warmer than the thermocline waters. The surface waters were particularly warm at the Almería-Oran front during the IRENE cruise in July 2017. A deep chlorophyll maximum was present within the thermocline (Figure F-4). During the springtime cruise (CLP19), the thermocline density surfaces were outcropping in a 50-70 m deep mixed layer and the chlorophyll concentrations were highest within the mixed layer in the Atlantic and Modified Atlantic waters with a deep chlorophyll maximum only in the Mediterranean water masses. The thermocline outcropping opens the possibility for water mass exchange between the sea surface and the interior. The deeper mixed layer offers the possibility of enhanced submesoscale dynamics. The thermocline density structure and 

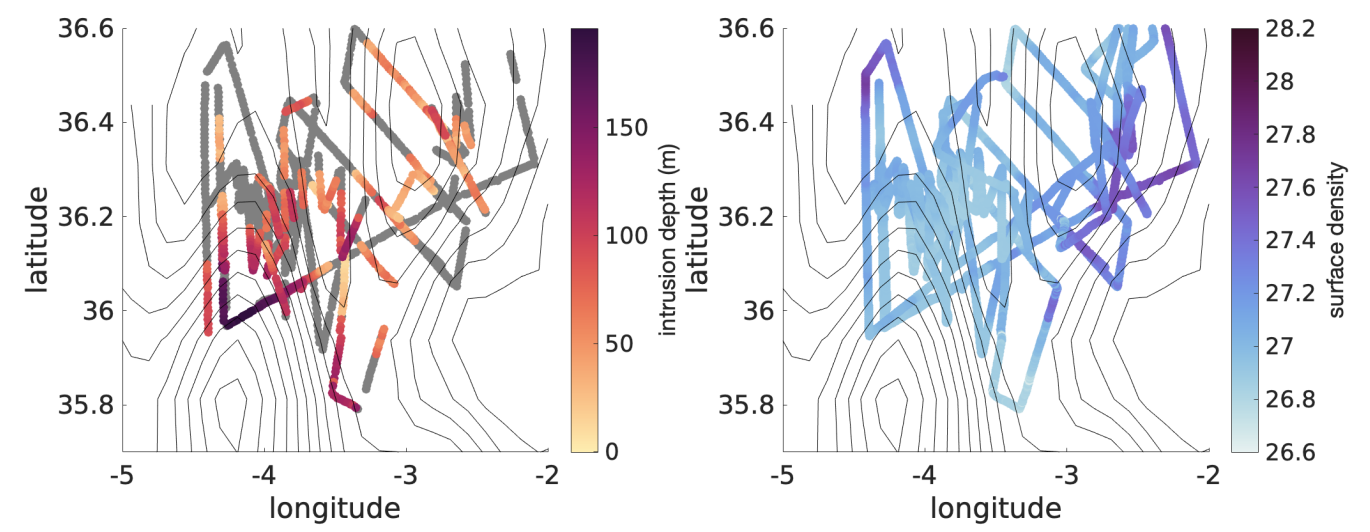

Figure 5-3: Distribution of intrusions in space during CLP19. The contours are sea surface height from AVISO. The Western Alborán Gyre is in the lower left and the anticyclonic eddy is in the upper right. (left) The grey shows the location of all underway CTD samples. In each cast where a intrusion was identified from the temperature-salinity properties, the depth of the top of the intrusion water in meters is plotted in color. (right) Sea surface density on the cruise track from the seawater intake thermosalinograph.

\begin{tabular}{|c|l|l|l|c|l|}
\hline Name & Dates & $\begin{array}{l}\# \text { uCTD } \\
\text { profiles }\end{array}$ & $\begin{array}{l}\# \text { CTD } \\
\text { casts }\end{array}$ & average MLD & features sampled \\
\hline IRENE & July 17-24, 2017 & 88 & 9 & 9.6 & $\begin{array}{l}\text { Almería-Oran } \\
\text { front }\end{array}$ \\
\hline CLP18 & $\begin{array}{l}\text { May 27-June 2, } \\
2018\end{array}$ & 819 & 17 & 19.4 & $\begin{array}{l}\text { Western edge of } \\
\text { Eastern Alborán } \\
\text { Gyre }\end{array}$ \\
\hline CLP19 & $\begin{array}{l}\text { March 21-April } \\
12,2019\end{array}$ & 1809 & 43 & 56 & $\begin{array}{l}\text { Western Alborán } \\
\text { Gyre and anticy- } \\
\text { clonic eddy }\end{array}$ \\
\hline
\end{tabular}

Table 5.1: Overview of research cruises. Average MLD is the average mixed layer depth in the CTD cast density profile.

$\theta$-S properties did not vary between seasons (research cruises). The maximum chlorophyll concentration was within the same temperature and same density range throughout the year (Figure 5-2). The density range of the chlorophyll maximum also corresponds to the nitracline density range. Nitrate is a primary limiting nutrient in this environment. The nitracline is on a lighter density surface in the Atlantic Water and a denser surface in the Mediterranean Water. The nitracline (region of rapid increase in nitrate concentrations) was at 50-100 $\mathrm{m}$ and surface nitrate concentrations were $0-1 \mu \mathrm{M}$ with higher concentrations in the Atlantic waters that had deep mixed layers during CLP19. The 1\% light level was at 50-70 m. 


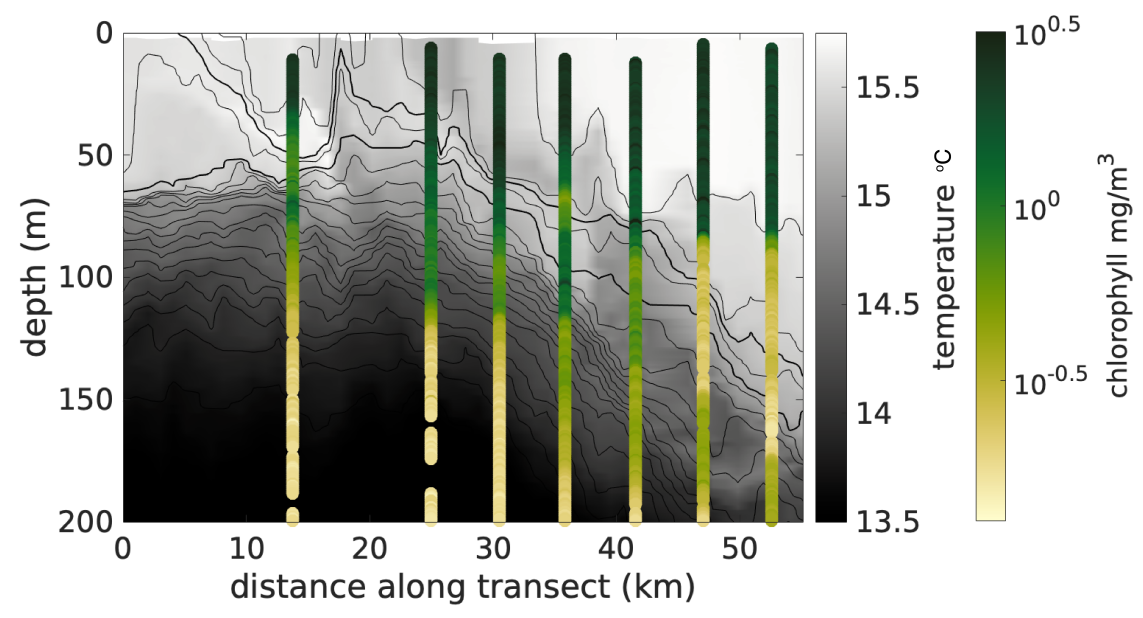

Figure 5-4: Transect C2, as labeled on figure 5-1. The background shows the temperature from an underway CTD transect immediately prior to the CTD transect. The black contours are density. The chlorophyll concentration shown is from the CTD casts. The uCTD and CTD transects were a few hours apart so the features in temperature and chlorophyll are not perfectly aligned in depth due to internal waves and tides.
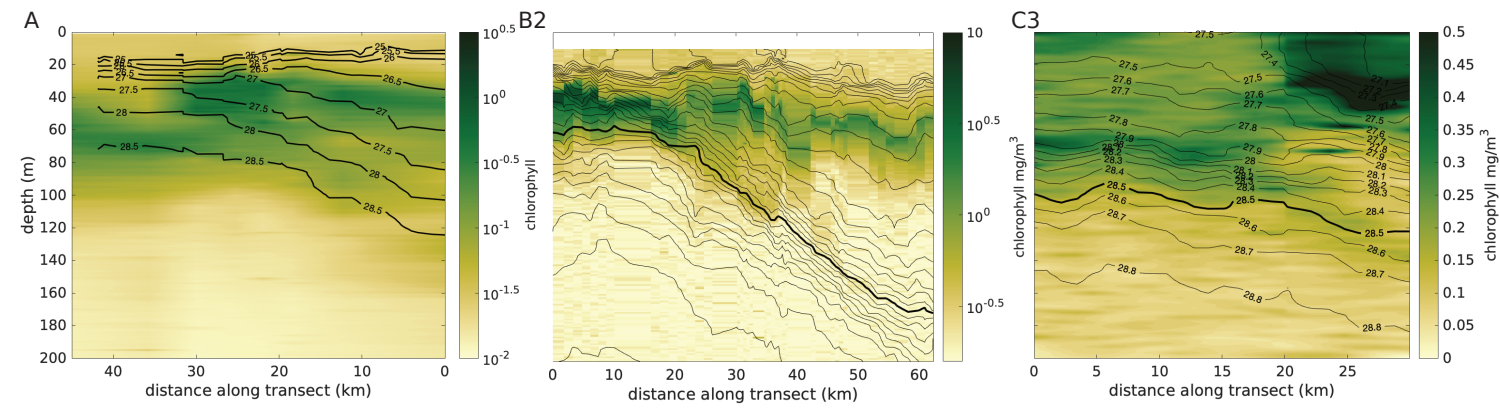

Figure 5-5: Transects A, B2, and C3, as labeled on figure 5-1. Chlorophyll concentration from the EcoCTD. The density contours on sections B2 and C3 are $0.1 \mathrm{~kg} / \mathrm{m}^{3}$ apart. The thick density contours show the density of the intrusion observed in the transect. 


\subsection{Observations of subduction}

During each research cruise, we measured vertical profiles of the hydrographic, biogeochemical, and biological properties on transects across fronts. Profiles were obtained using towed profiling instruments, the underway CTD (uCTD) and EcoCTD, and were closely spaced, at approximately $1 \mathrm{~km}$ distance between profiles, to observe variability at the submesoscale. Both the uCTD and EcoCTD are towed profilers that are operated from the ship with a winch while underway. All of the 2410 uCTD profiles have temperature, salinity, and pressure observations. The 561 profiles taken with the EcoCTD also have oxygen, chlorophyll, and backscatter (Figure 5-5) (Dever et al., 2020). The sampling was carried out adaptively to target subduction at the submesoscale $(1-10 \mathrm{~km})$. We used satellite and model observations in combination with gyre-scale ship surveys and drifter releases to locate mesoscale and submesoscale fronts. We then performed intensive surveys of the frontal regions (Figure $5-3)$.

In the cross-front surveys we observe thermohaline intrusions (Shcherbina et al., 2009), which appear as inversions in temperature or salinity in a vertical profile (or in density coordinates). Intrusions are generated by the stirring of water masses across the front. These intrusions may also appear as a local maximum or minimum in biogeochemical properties. Throughout this chapter, we will refer to these features as "intrusions", a term which here encompasses both thermohaline and biogeochemical intrusions. In the case of the biogeochemical intrusions, which are the focus on this chapter, they are also thermohaline intrusions. While intrusions are identified in vertical profiles (Ruddick and Richards, 2003), they are the result of lateral transport on density surfaces. Due to the high lateral resolution of our sampling, we are able to capture multiple observations of a single intrusion feature in a cross section across the three-dimensional feature. Using these observations, in addition to discussing the ways that these intrusions result in anomalous profiles in the vertical, we emphasize their role as pathways of material transport both vertically and laterally.

The distribution of watermass properties (temperature and salinity) on a density surface is the outcome of physical stirring and mixing. The variance of temperature and salinity on a density surface, or a combination of the two variables called spiciness (McDougall et al., 
2021) can be used to make inferences about the dynamics that led to that distribution (Kunze et al., 2015; Sprio Jaeger et al., 2020). The stirring process generates variance in spice through filamentation. This variance is often parameterized as diffusive mixing. If we normalize the variance by the gradient on which the stirring operates, we obtain the typical length scale of these features, which is called a mixing length $(\lambda)$ (Cole et al., 2015).

$$
\lambda=\frac{\left\langle\Pi^{\prime} \Pi^{\prime}\right\rangle}{\langle|\overline{\nabla \Pi}|\rangle}
$$

where $\Pi^{\prime}$ is the spice anomaly from a regional mean and $|\overline{\nabla \Pi}|$ is the magnitude of the spice gradient on isopycnal surfaces on each transect. The angled brackets indicate averages over the whole region. This computation is done in isopycnal coordinates. The average gradient is computed for each transect as a linear fit of spice with distance on a density surface. The mixing lengths, approximately $20 \mathrm{~km}$, correspond well to the lateral scales of the intrusions (Figure 5-6). These values are slightly smaller than the range of the mixing lengths obtained by (Cole et al., 2015) on much larger scales $\left(3^{\circ}\right.$ by $3^{\circ}$ grid $)$.

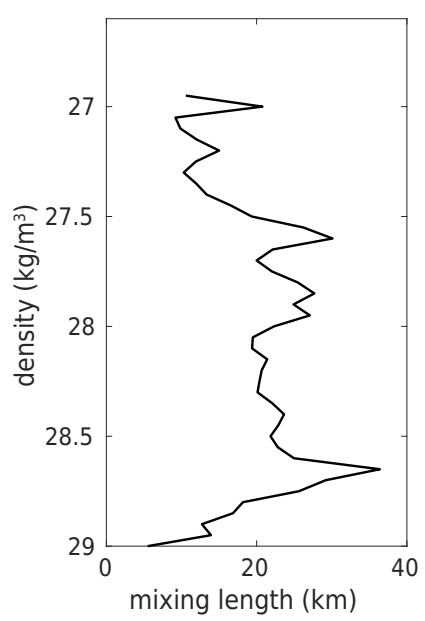

Figure 5-6: Average mixing length calculated on each cross-front transect using the spice variance on density surfaces.

At the fronts the density surfaces are steeply sloping. Therefore, along isopycnal motion will have a vertical component, which generates subduction (Freilich and Mahadevan, 2019). We observe subduction across the full depth range of the frontal density surfaces from the surface down to $200 \mathrm{~m}$, the maximum profile depth. There are numerous physical 
mechanisms that can result in subduction at fronts and filamentation of tracers along density surfaces including geostrophic frontogenesis and quasigeostrophic stirring (Bower, 1991; Spall, 1995; Cole and Rudnick, 2012), submesoscale mixed layer processes (Erickson and Thompson, 2018), coupled mesoscale-submesoscale processes (Callies and Ferrari, 2013; Ramachandran et al., 2014; Freilich and Mahadevan, 2021), wind forcing, internal wave strain, and a combination of multiple physical dynamics (Klymak et al., 2015; Kunze et al., 2015; Spiro Jaeger et al., 2020). The intrusions that we observe are likely generated by a range of distinct mechanisms. The cross front transects sampled during the three research cruises show that thermohaline and biogeochemical intrusions can result in somewhat distinct appearances across the front, likely due to variation in the generation process (Figures 5-5 and 5-4). In Figure 5-4 we show that the thermohaline and biogeochemical intrusions co-occur.

In a process study model based on this region, the dominant process for water parcels leaving the mixed layer was geostrophic frontogenesis, with contributions from ageostrophic frontogenesis mechanisms as well. This means that there is a predominance of the influence of the mesoscale front on the subduction process with contributions from submesoscale processes as well. Once water parcels leave the mixed layer, the subducted water is stirred along sloping isopycnal surfaces and may continue to subduct (Freilich and Mahadevan, 2021). This process study model represents the conditions during CLP19 when the thermocline outcropped in the mixed layer and the biomass was most concentrated in the mixed layer. When the surface is stratified and there is a deep biomass maximum, stirring on sloping isopycnal surfaces (previously been described as a cross-front exchange process) can also result in subduction (Bower, 1991). At times, we observe that intrusions appear to be crossing isopycnal surfaces. This is likely due to the subduction process taking place on a range of density surfaces (on a horizontal surface at a front there is a relatively large range of density in a small area). The tracer distribution generated by this subduction and stirring was described by Spiro Jaeger et al. (2020) in the mixed layer in the Bay of Bengal.

Due to the along-front current, water parcels are moving more quickly along-front than either downwards or across the front. Model analysis shows that water parcels come from 25-100 km upstream of the location where they are observed subsurface (Figure 5-7). In two process study models with the same thermocline density structure but distinct near surface 

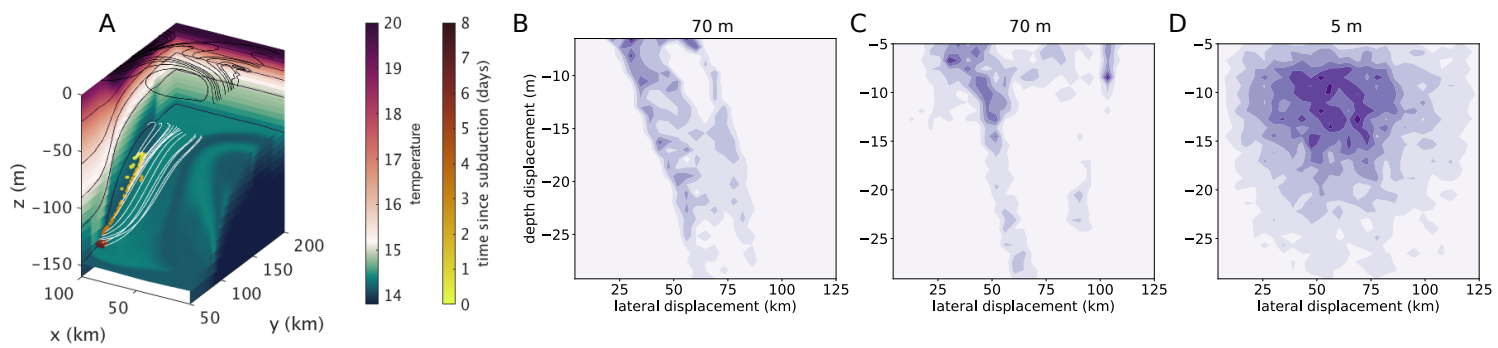

Figure 5-7: Model analysis of subduction. (A) Lagrangian particles (yellow) originated at $70 \mathrm{~m}$ and are subducted along an isopycnal surface. The white trajectories show the $x-y-z$ location history of the particles. The isopycnal surface is shown with temperature shading. Temperature is shown on the boundaries of the frame as well. (B-D) Histograms of the relationship between vertical and lateral displacement of water parcels. (B) particles initially at $70 \mathrm{~m}$ in a model with a stratified surface layer representing the June and July research cruises. The particles shown in panel (A) are a subset of the particles shown in panel B. (C) particles initially at $70 \mathrm{~m}$ in a model with $50 \mathrm{~m}$ deep mixed layer representing the March-April research cruise. (D) particles initially at $5 \mathrm{~m}$ in the model with a $50 \mathrm{~m}$ deep mixed layer. Statistics on this plot are from water parcels that leave the mixed layer.

stratification (one stratified as in IRENE and one with 50-70 m deep mixed layers as in CLP19), there is a positive relationship between lateral and vertical distance on water parcels that subduct within the thermocline that corresponds approximately to the isopycnal slope in the thermocline (Figure 5-7B,C). Water parcels that are deeper have traveled farther from their origin location both in the horizontal and vertical with the ratio of those two motions set by the isopycnal slope. In these models, the water parcels that originate within the thermocline experience subduction along a well-defined isopycnal surface and their motions show the signature of the mesoscale meander (Bower, 1991). The relationship between vertical and lateral motions is less coherent for water parcels that subduct from the mixed layer where the isopycnal slope is less well-defined and there is a greater influence of smallscale processes (Figure 5-7D; (Freilich and Mahadevan, 2021)).

On selected transects where we observed a thermohaline intrusion, which were ubiquitous near the fronts (Figure 5-3), or when using the EcoCTD, where we observed a biogeochemical intrusion, we performed additional sampling with a CTD rosette from vertical casts in a transect across the front. These transects allowed us to carry out more detailed observations of the biological and biogeochemical properties using optical instruments mounted to a CTD rosette and by sampling the water to $250 \mathrm{~m}$ depth. We performed $68 \mathrm{CTD}$ casts arranged in 13 transects. We spaced the casts closer together at the center of the front to capture the 
subduction region and extended the casts towards the light side of the front to capture the lateral extent of the features. These transects reveal that the thermohaline intrusions can also be associated with high chlorophyll concentrations (Figures 5-4 and 5-5). In a vertical profile, the result is a secondary chlorophyll maximum that can be below the euphotic zone, particularly on the light side of the front. The secondary chlorophyll maximum is also often associated with low apparent oxygen utilization (AOU), low nutrients, high POC, and anomalous water mass properties (typically warmer and saltier due to its origins on the dense side of the front). The intrusions are observed consistently across the front supporting the hypothesis that they are the result of an advective subduction pathway rather than in situ growth. In addition, each intrusion has nearly constant density suggesting a mostly along isopycnal subduction and stirring process. At each CTD station, we picked discrete water sample depths using an adaptive sampling procedure. We sample 6 depths: the surface, chlorophyll maximum, and secondary chlorophyll maximum if it is present and waters with low chlorophyll within and below the euphotic zone, which we term "background" samples. In the analysis presented here, intrusions must satisfy three criteria to be included in the analysis.

I. Intrusions are identified as a secondary chlorophyll maximum. The chlorophyll maximum must extend at least $5 \mathrm{~m}$ in the vertical. The bounds of the intrusion are set by the bounds of the elevated chlorophyll concentration. There are thermohaline intrusions that are not associated with elevated chlorophyll concentration. These are intrusions in a more general sense but are not included in the analysis here because they do not necessarily contribute more to carbon export than the background waters. Within an intrusion, there also may be vertical gradients in chlorophyll because the chlorophyll and temperature-salinity gradients are not aligned. There are also some profiles with a secondary maximum in POC that does not have an elevated chlorophyll signal. We do not include those in the analysis because we did not sample their composition and so cannot be sure that the POC is microbial POC. Future work could examine these features to ascertain the amount and composition of the POC in high POC, but low chlorophyll intrusions. 
II. Intrusions are present continuously in more than one CTD cast within a transect. The intrusion must be below $100 \mathrm{~m}$ in at least one cast along the transect. Being present continuously means that the intrusion is present within the same density range across the front. This criteria allows us to confidently identify narrow intrusions in terms of their vertical extent over large lateral scale. In addition, we are able to identify the origins of the intrusions within the euphotic zone by identifying the intrusions when they are above $100 \mathrm{~m}$. By contrast, when identifying intrusions from isolated profiles Llort et al. (2018) were only able to identify intrusions that were already below $100 \mathrm{~m}$ and that were at least $150 \mathrm{~m}$ in vertical extent.

III. The chlorophyll maximum is associated with an inversion in the vertical in at least one other property (temperature, salinity, oxygen, POC). This criteria helps to ensure that the secondary chlorophyll maximum is due to an intrusion rather than local growth or sinking of phytoplankton.

\subsection{Biophysical determinants of carbon flux}

\subsubsection{Magnitude of carbon subduction}

Collectively, the above outlined observations reveal that frontal subduction provides pathways for the transport of photosynthetically generated particulate organic carbon (POC) and associated heterotrophic productivity from the surface ocean to the interior. The intrusions in the Western Alborán Gyre have twice as much POC as the background with the same depth distribution (Table 5.2). In this analysis, intrusions are identified by their fluorescence signal for consistent comparison with biological communities that were sampled at discrete locations and will be discussed in the following sections. There is, however, enhanced POC and oxygen at other depths and thermohaline inversions that are not classified as "intrusions" in this analysis. There is a greater volume of water in intrusions at shallower depths (Figure 5-8). Because intrusions are identified based on the biological signatures, the identified volume decays with depth due to both physical and biological processes including a decay of chlorophyll per cell when below the euphotic zone, remineralization of the 
organic matter, mixing of the intrusion water with the surrounding water, and a decay in the vertical velocity magnitude with depth. The POC concentration is estimated from $c_{p}$ at $0.5 \mathrm{~m}$ resolution on each CTD cast. To compare the intrusion POC concentration with the background, the background POC concentration is estimated by subsampling the POC concentration outside of the intrusions with the same depth distribution as the intrusions. Vertical motion results in a POC flux because there is a strong vertical gradient in POC with depth. During CLP19, the POC on average decreases with depth with a rapid decrease in POC concentration centered at $60 \mathrm{~m}$ (Figure 5-8). The average POC concentration in the intrusions has the same value as the average POC profile $45 \mathrm{~m}$ shallower.

We can estimate the magnitude of the POC flux locally from the observations. For example, on transect C2 (Figures 5-4), the POC flux within the intrusion is on the order of $10 \mathrm{mmol} \mathrm{m}{ }^{-2}$ day $^{-1}$ given the observed magnitude of POC anomaly $\left(\sim 1 \mathrm{mmol} / \mathrm{m}^{3}\right)$ and estimating the vertical velocity magnitude as approximately $10 \mathrm{~m} /$ day. This vertical velocity estimate is fairly conservative because it neglects mixing. We observe a cross-frontal velocity of $0.05 \mathrm{~m} / \mathrm{s}$ in section $\mathrm{C} 2$ and the isopycnal slope is 0.005 (150 m depth over $30 \mathrm{~km}$ ). The vertical projection of an along isopycnal cross-front velocity is therefore $20 \mathrm{~m} /$ day. Another method for estimating the timescale is to use the AOU as a tracer of the time since subduction. Within all intrusions, the AOU increases and POC decreases with depth. We assume that water parcels that are shallower in the intrusions have subducted more recently (Figure 5-7A). We then make the assumption that the majority of the increase in AOU is attributable to respiration and use the dark respiration rate measured using the autoBOD to obtain a timescale. In the intrusion we observe respiration rates of $-2.02 \mu \mathrm{mol} / \mathrm{L} /$ day near $70 \mathrm{~m}$ and $-0.26 \mu \mathrm{mol} / \mathrm{L} /$ day near $180 \mathrm{~m}$. Given the change in oxygen of $50 \mu \mathrm{mol} / \mathrm{L}$ along the intrusion and using the larger respiration rate, the subduction to $180 \mathrm{~m}$ is estimated to have taken 25 days, which would imply a downward rate of movement of $6 \mathrm{~m} /$ day. There may be other sinks of oxygen at depth. Mixing is a likely sink but the along isopycnal mixing rates are difficult to constrain. If we account for mixing, the timescale of the subduction event would be shorter.

The contrast in biogeochemical properties between watermasses affects the carbon subduction. Within the surface layer, the POC is patchy and depends on the watermass. The 


\begin{tabular}{|c|ccc|ccc|}
\hline location & \multicolumn{3}{|c|}{ POC } & \multicolumn{3}{c|}{ AOU } \\
& intrusions & background & difference & intrusions & background & difference \\
\hline CLP19 WAG & {$[2.35,2.44]$} & {$[1.39,1.48]$} & 0.9 & {$[41.9,43.4]$} & {$[46.1,48.9]$} & 4.3 \\
CLP19 eddy & {$[1.33,1.38]$} & {$[1.25,1.31]$} & 0.1 & {$[36.6,39.9]$} & {$[48.5,50.8]$} & 11 \\
CLP18 & {$[3.27,3.45]$} & {$[1.30,1.52]$} & 2 & {$[56.9,59.2]$} & {$[64.1,67.8]$} & 8.4 \\
\hline
\end{tabular}

Table 5.2: POC concentration $(\mu \mathrm{mol} / \mathrm{kg})$ and AOU $(\mu \mathrm{mol} / \mathrm{kg})$ in intrusions below $100 \mathrm{~m}$ and outside the intrusions ("sampled background"). The intervals show the bootstrapped $95 \%$ confidence interval of the geometric mean of each category (1000 iterations). The concentration outside the intrusions is calculated by averaging the POC concentration from a random sample of points with the sample depth distribution as the intrusion samples. The variation in the geometric mean and the confidence intervals among random samples from the background is less than $0.001 \mu \mathrm{mol} / \mathrm{kg}$. The location is the research cruise and region. CLP19 WAG is transects C1, C2, and C5 as well as the casts between C1 and C2 where only nutrients are sampled. CLP19 eddy is transects C3 and C4 and the transect near C3 with only nutrients sampled. CLP18 is transects B1 and B2. The t-test p value that the geometric mean in the intrusions is significantly different from the sampled background is less than 0.001 in all cases.

denser Mediterranean water, which is more oligotrophic, has lower POC concentrations in the intrusions. This watermass makes up the waters in the low POC peak in the histogram in figure 5-8a. The intrusion in the anticyclonic eddy sampled during CLP19 has only a slightly larger POC concentration in the intrusions relative to the background. In cases where dense water has higher biomass, this subduction mechanism could be even stronger. In the Alborán Sea, the fronts are known to enhance productivity so frontally-driven export is operating in locations with already enhanced POC concentrations and can serve to propagate the signal of frontal surface production to depth (Gorsky et al., 2002).

\subsubsection{Contribution to export by small cells}

The POC in the intrusions is mostly picophytoplanktonic and heterotrophic bacterial cells. There is a strong linear correlation between the total POC estimated by the transmissometer and the POC measured by flow cytometry (Figure 5-10). This, coupled with the fluorescence signal, is evidence that the subducted carbon is in large part microbial POC. By comparing biomass computed from cell counts with taxa-specific cell carbon estimates and from beam-c measurements, we estimate that overall small cells contribute $10-55 \%$ of the POC. Heterotrophic bacteria make up about half of the microbial POC estimated from cell counts, due to their large numbers, despite their small size. The cell abundance to carbon 


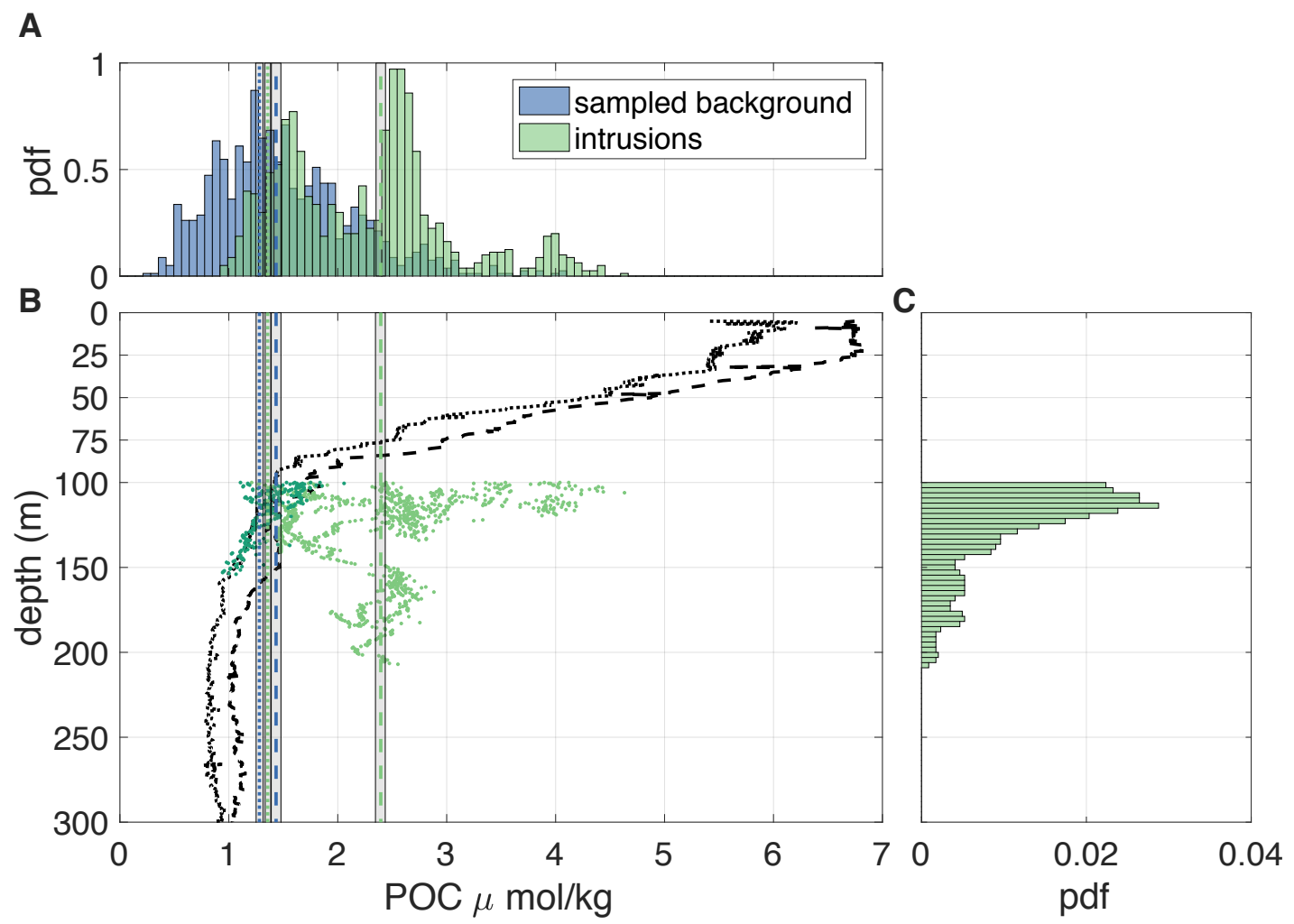

Figure 5-8: POC export during CLP19. (A) Probability density functions of POC concentration within the intrusions (green) and POC concentration randomly sampled with the same depth distribution as the intrusions but outside the intrusions. The dashed line shows the geometric mean of the POC distribution in the gyre intrusion and background. The dotted line shows the mean of the POC distribution in the eddy intrusion and background. The grey shading shows the $95 \%$ confidence interval. These averages are also shown in panel (B). (B) Average POC profile in the gyre (dashed) and eddy (dotted). The green scatter points show the intrusion POC concentrations at each depth below $100 \mathrm{~m}$. The darker points are in the eddy and the lighter points are in the gyre. (C) Depth distribution of intrusion samples below $100 \mathrm{~m}$.

biomass conversions used from past studies are highly variable (Buitenhuis et al., 2012). However, the conversion from transmissometer $c_{p}$ to POC is less variable and the strong linear relationship between $c_{p}$ and carbon estimated from cell counts provides evidence that the POC has microbial sources.

\subsubsection{Community composition}

The depth dependent POC concentration differentiated by microbial community composition are shown in Fig. 5-11. During IRENE, the cell counts of Synechococcus, high-light 


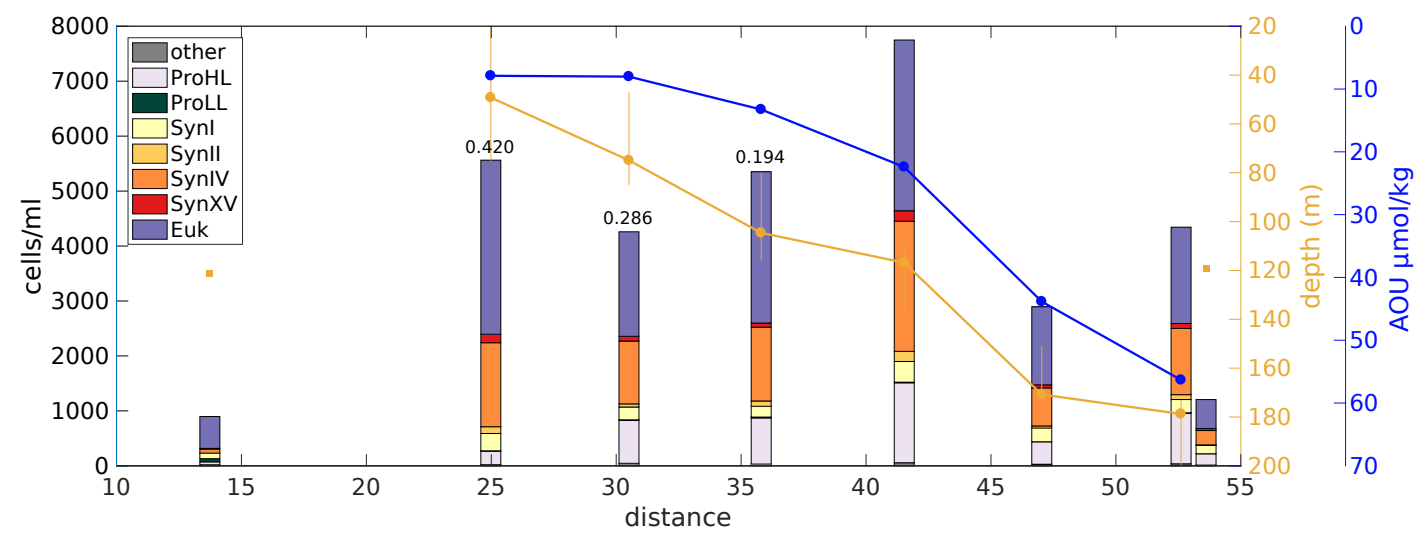

Figure 5-9: Samples from the intrusion in transect C2 (Figure 5-1). This transect cross section is shown in figure 5-4. In the cross section, the intrusion samples are shown in black dots. The black squares show the samples on the left and right sides of this figure, which are outside of intrusions and considered "background". The cell concentrations are derived from flow cytometry. The cell concentrations of the cyanobacteria ecotypes are estimated by mapping the relative abundance of ecotypes onto cell counts from flow cytometry. The yellow line shows the intrusion depth with the dot indicating the sample location and the bars showing the feature depth range. The blue line shows the average AOU within the intrusion. The numbers above the bars near 25, 30, and $35 \mathrm{~m}$ are $\mathrm{F}_{\mathrm{v}} / \mathrm{F}_{\mathrm{m}}$ measurements for those samples.

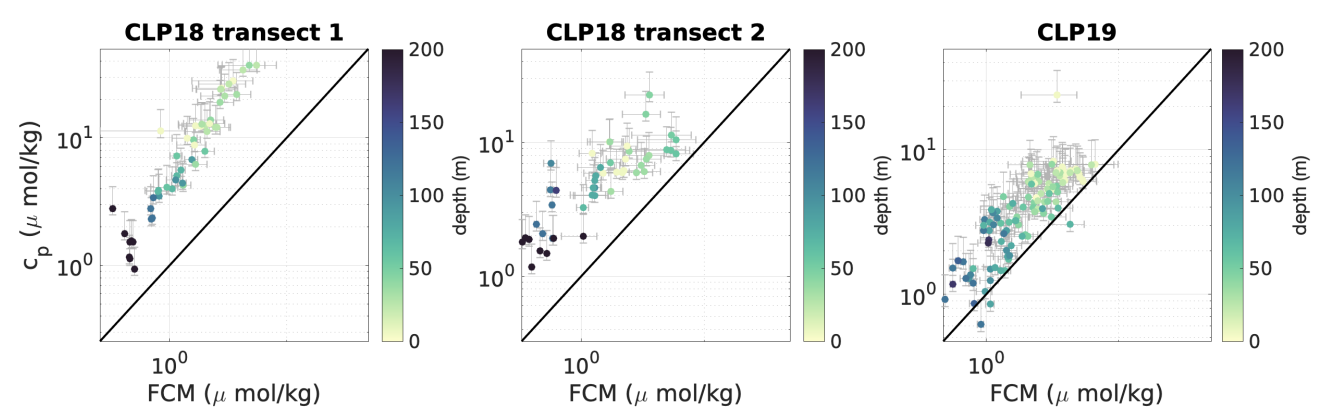

Figure 5-10: POC estimated from flow cytometry, which captures the small photosynthetic and heterotrophic biomass plotted against the POC estimate from beam transmission, which captures a larger fraction of the total POC and non-biological particulate matter. The grey bars show the ranges of possible values based on the uncertainty in the conversion factor. The uncertainty is correlated between points. The first CLP18 transect was more coastal, ending near Moroccan territorial waters, which could explain the relatively low fraction of the POC that is in the small fraction. The black line is the 1:1 line. 

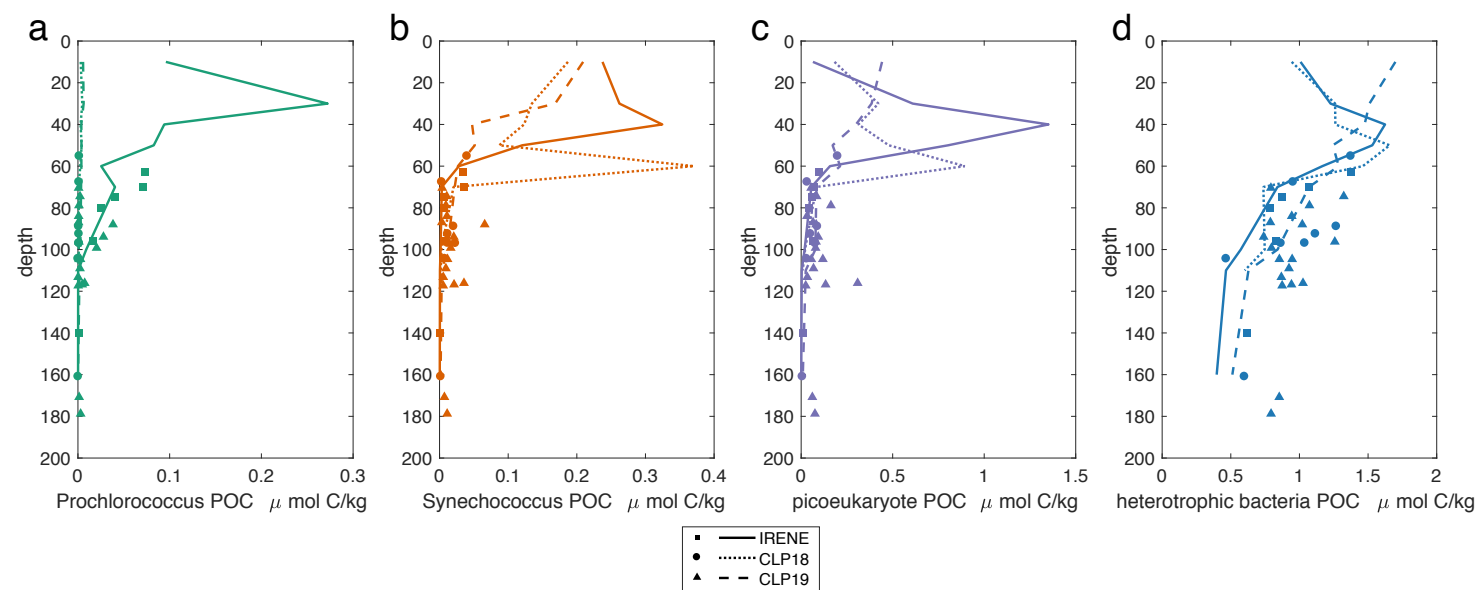

$$
\begin{array}{|l|}
\hline:- \text { IRENE } \\
:-- \text { CLP18 } \\
\hline-- \text { CLP19 } \\
\hline
\end{array}
$$

Figure 5-11: Profiles of the average POC concentration in each taxonomic group estimated from flow cytometry, separated by sampling year (lines). The scatter points show the POC concentration in the intrusions.

strains of Prochlorococcus, and of picoeukaryotes are highest near the surface with high cell counts of Synechococcus at the surface and the maximum counts of picoeukaryotes slightly deeper. During CLP18, cell counts peaked lower in the water column with a Synechococcus peak at the surface in some profiles. There was almost no Prochlorococcus present during CLP18. During CLP19, the peak in the cell counts was at the surface for almost all groups. Prochlorococcus was low in abundance overall but was observed in the anticyclonic eddy. The heterotrophic bacteria cell abundance peaked slightly shallower than the photosynthetic cell counts (Figure 5-11d). Recent work has shown that the deep biomass maxima observed in the Western Mediterranean Sea in May and June are also the locations with the maximum primary production and bacterial production (Marañón et al., 2021). The maximum bacterial production and bacterial abundance occur shallower than the primary production maximum, as observed by Marañón et al. (2021). The depth structure is set by both biological and physical factors. The vertical structure of photosynthetic communities is set by the distinct light and nutrient requirements of the different phytoplankton types, which differ physiologically (Marañón et al., 2021).

The community composition has large gradients in the vertical, and laterally across the fronts. Because vertical advection of organic carbon results from the co-occurrence of vertical velocities with biomass, the depth structure of the community has implications for the composition of the POC flux. Phytoplankton and bacterioplankton species have different 


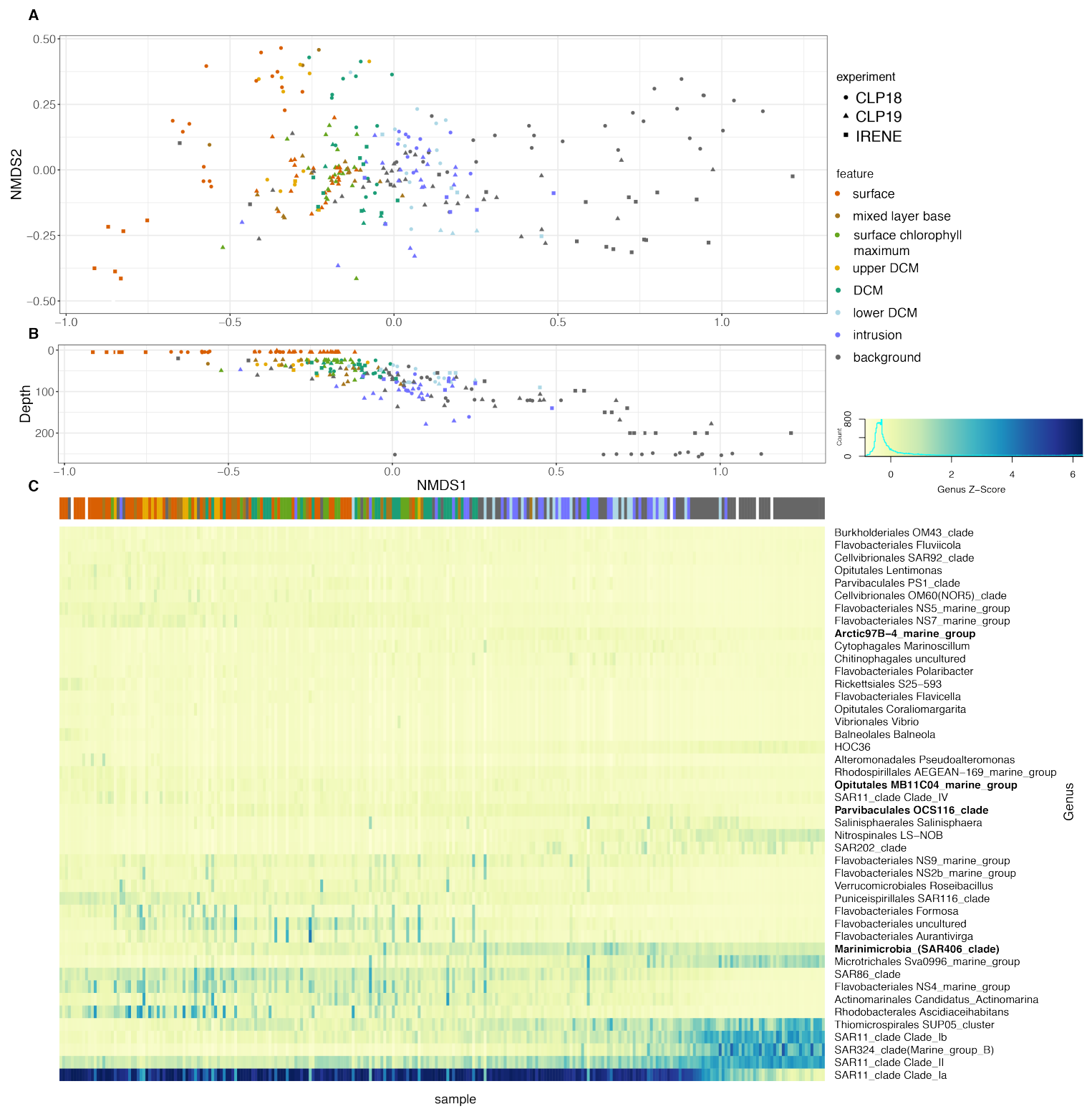

Figure 5-12: Ordination of heterotrophic bacteria ASVs. (A) Non-metric multidimensional scaling (NMDS) of the Bray-Curtis distance between samples. The ordination used 3 NMDS axes (only two are shown) and resulted in a stress of 0.071 . The sample feature is shown with the color of the point and the research cruise is shown in the shape of the point. (B) The first NMDS axis is plotted against the sample depth. (C) ASVs are aggregated at the genus level and all genera that comprise at least $2 \%$ of one sample are plotted. The color in the heatmap shows the z-score of the relative abundance within the sample. Samples are arranged in the order of the first NMDS axis. 


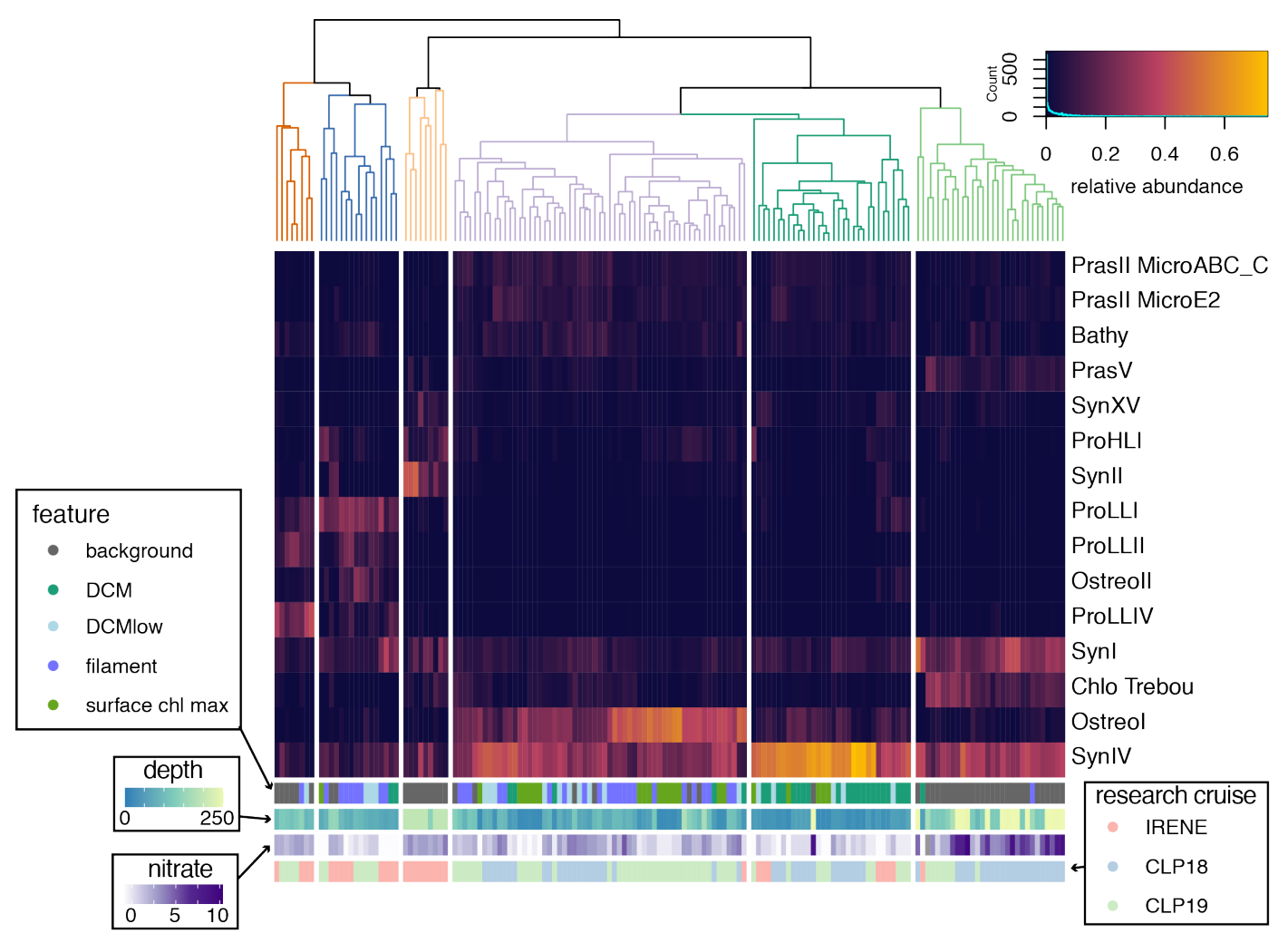

Figure 5-13: Hierarchical clustering of samples from the intrusions, background below $90 \mathrm{~m}$, surface chlorophyll maximum, deep chlorophyll maximum, and lower deep chlorophyll maximum. Sample distance is Bray-Curtis distance and clusters are formed using average-linkage clustering. The first bar below the tree shows the feature that the sample came from, the second shows the sample depth, the third shows the sample nitrate concentration, and the fourth shows which research cruise. The heatmap shows the relative abundance of the 15 most common taxa in these samples.

elemental compositions, which means that the nutritional contribution to the mesopelagic due to exported carbon depends on which species are exported (Finkel et al., 2010). The dependence of the community composition on water mass also has implications for subduction because we mostly see subduction from the denser water masses, the Modified Atlantic Water and the Mediterranean Water.

Depth is the main axis of organization of both the heterotrophic and small photosynthetic community composition at the broad taxonomic level inferred from flow cytometry and at the finer taxonomic resolution provided by $16 \mathrm{~S}$ amplicon sequencing. The dominant driver of similarity in community composition between samples is the sample depth. We quantify similarity between samples using the Bray-Curtis dissimiliarity based on ASVs (exactly identical sequences obtained from amplicon sequencing) in the case of the hetertrophic 
bacteria and ecotypes of Prochlorococcus, Synechococcus, and Viridiplantae derived from Phyloassigner in the case of the small photosynthetic community. The first NMDS axes of the heterotrophic bacteria 16S ASVs and combined Cyanobacteria and Prasinophyte composition are highly correlated with depth $\left(r^{2}=0.76\right.$ and $r^{2}=0.66$, respectively) (Figures 5-12 and F-8). The second NMDS axis in both cases has a clear relationship with watermass structure. These observations are consistent with observations from other oligotrophic systems such as the subtropical Atlantic that show that bacterioplankton community composition depends strongly on depth and water mass (Treusch et al., 2009; Agogué et al., 2011; Choi et al., 2020). Notably, we observe that the samples from the three research cruises cluster together with differences attributable to water mass. In a heirarchical clustering analysis of the photosynthetic communities (Cyanobacteria and Viridiplantae), the samples from the Mediterranean waters form a cluster that is distinct from the Atlantic and Modified Atlantic waters (Figure 5-13; orange and blue clusters).

The vertical partitioning of the bacterioplankton communities is fairly typical of stratified oligotrophic regions. The dominant heterotrophic bacterioplankton in the surface samples is SAR11 clade Ia. At depth, the dominant taxa are SAR11 clades Ib and II and Deltaproteobacteria SAR324 which are known to be abundant in the subtropical Atlantic mesopelagic (Wright et al., 1997; Carlson et al., 2009; Vergin et al., 2013a) as well as Thiomicrospirales (SUP05 cluster) and Microtrichales (Sva0996 marine group). Thiomicrospirales has been observed to be relatively abundant at depths greater than $80 \mathrm{~m}$ in the Monterey Bay (Reji et al., 2020) and the Sva0996 marine group has been observed in both sediments and the open ocean, including in the California Current, and plays an important role in dissolved protein utilization (Orsi et al., 2016).

This distribution of biomass and community composition in the euphotic zone is a major factor in the composition of the intrusions. Across all the transects, we find that heterotrophic bacteria make the largest contribution to the subducted POC, followed by picoeukaryotes (Figures 5-14, 5-16). Cyanobacteria make a larger contribution during the stratified summer period than in the springtime. Consistent with the enhanced POC in intrusions relative to the background, there is also a greater concentration of cells from all taxonomic groups identified by flow cytometry relative to the background. This finding is 

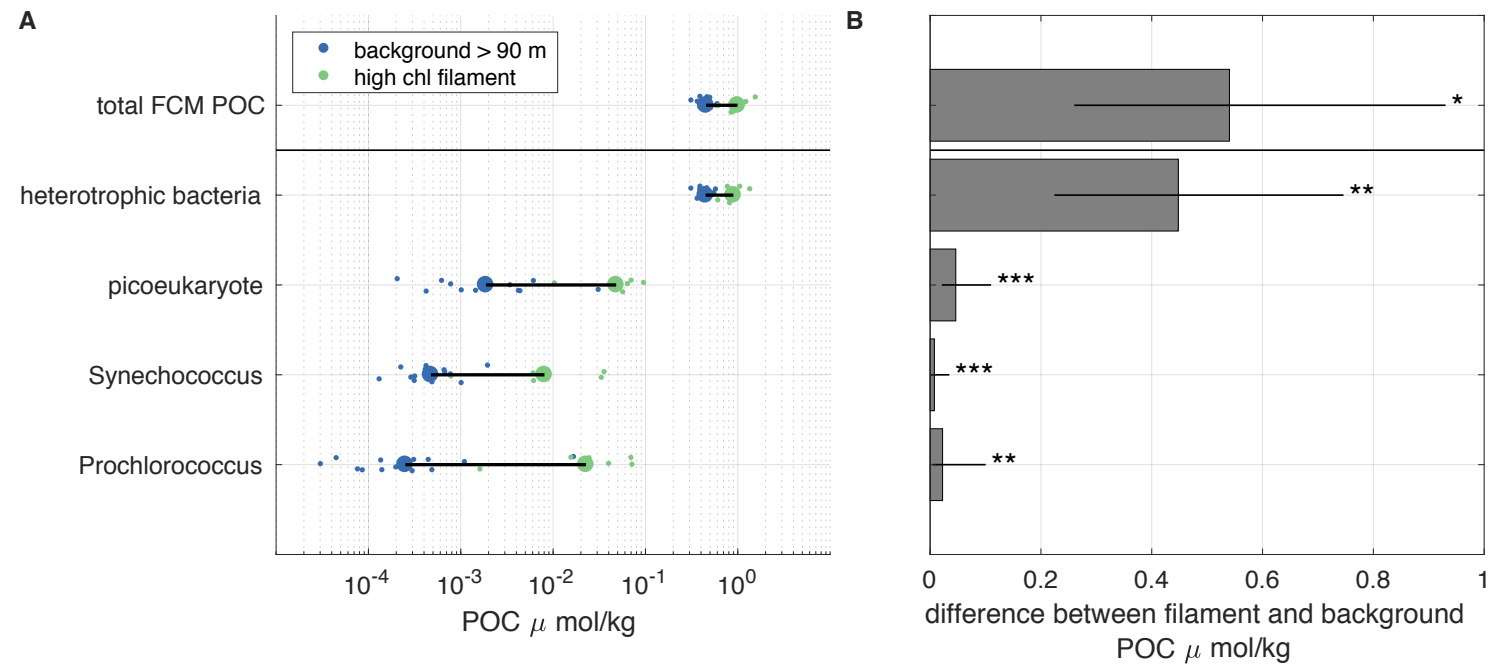

Figure 5-14: Observations of a intrusion at the Almeria-Oran front sampled during IRENE in July 2017. (A) POC concentration in each taxonomic group in intrusions (green) and background samples below $90 \mathrm{~m}$ (blue). (B) Difference between the geometric mean of intrusion and background POC concentrations in each taxonomic group and the total POC concentration estimated from flow cytometry. The t-test significance level of enhancement in the intrusion over the background is shown with the stars ${ }^{* * *}=\mathrm{p}<0.001$, ${ }^{* *}=\mathrm{p}<$ $0.01, *=\mathrm{p}<0.05$.

significant because previous work on carbon export has focused on the photoautotrophic community and neglected the contributions from the heterotrophic community or assumed that any increase in carbon is due to photoautotrophs (Omand et al., 2015; Siegel et al., 2016). While primary production supplies carbon to bacterial production, the standing stock of bacterioplankton cells, which are non-sinking, could be an important factor in the magnitude of carbon export. Given the depth structure of the communities, we expect that the communities in the intrusions will evolve from being dominated by surface taxa such as SAR11 clade Ia (Figure 5-12C) to mesopelagic taxa with potentially high mortality of the surface taxa. In sediment traps, community turnover can be rapid with deep taxa quickly colonizing particles (Fontanez et al., 2015). Additionally, the depth stratification of microbial communities results in variation in the community function with depth (DeLong et al., 2006). The organic matter composition also affects the bacterioplankton community composition (Stephens et al., 2020). The processing of organic matter supplied to the mesopelagic by a downward advective flux likely differs from the processing of sinking organic matter. The differences arise from the composition of the organic matter, ecological interactions with 

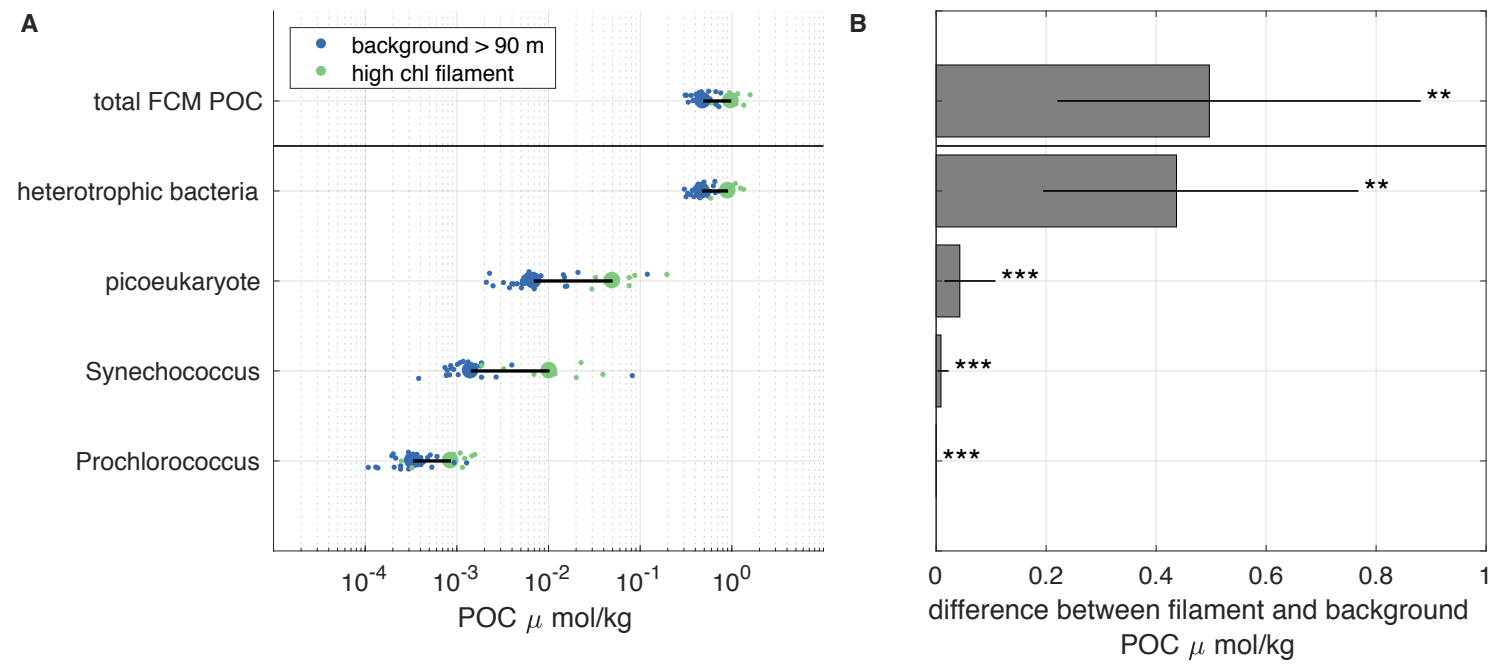

Figure 5-15: Observations of a intrusion at the Almeria-Oran front sampled during CLP18 in May-June 2018. (A) POC concentration in each taxonomic group in intrusions (green) and background samples below $100 \mathrm{~m}$ (blue). (B) Difference between the geometric mean of intrusion and background POC concentrations in each taxonomic group and the total POC concentration estimated from flow cytometry. The t-test significance level of enhancement in the intrusion over the background is shown with the stars ${ }^{* * *}=\mathrm{p}<0.001,{ }^{* *}=\mathrm{p}<$ $0.01,{ }^{*}=\mathrm{p}<0.05$.

the existing community, and the biogeochemical composition of the source seawater, which will be relatively high in oxygen and low in nutrients.

\subsubsection{Ecological effects of subduction}

\section{Dispersal}

To first order, the community composition within the intrusions reflects the origin communities. Therefore, one of the signals of subduction is anomalous community composition at a given depth, providing biological evidence of subduction. The photosynthetic communities in the intrusion samples cluster with the origin communities (Figure 5-13). In the samples from the stratified summertime experiments, the community composition is most like the lower DCM while for the springtime cruise, the water mass origins appear to be in the mixed layer. On average, the heterotrophic bacterial communities in intrusions are most similar to samples that are $17 \mathrm{~m}$ shallower than the sample location (t-test $\mathrm{p}<0.01,95 \%$ confidence interval [-27 -6.1]) (Figure 5-12). Only communities from the dense side of the front subduct due to eddy processes. This means that the majority of the communities from the chloro- 

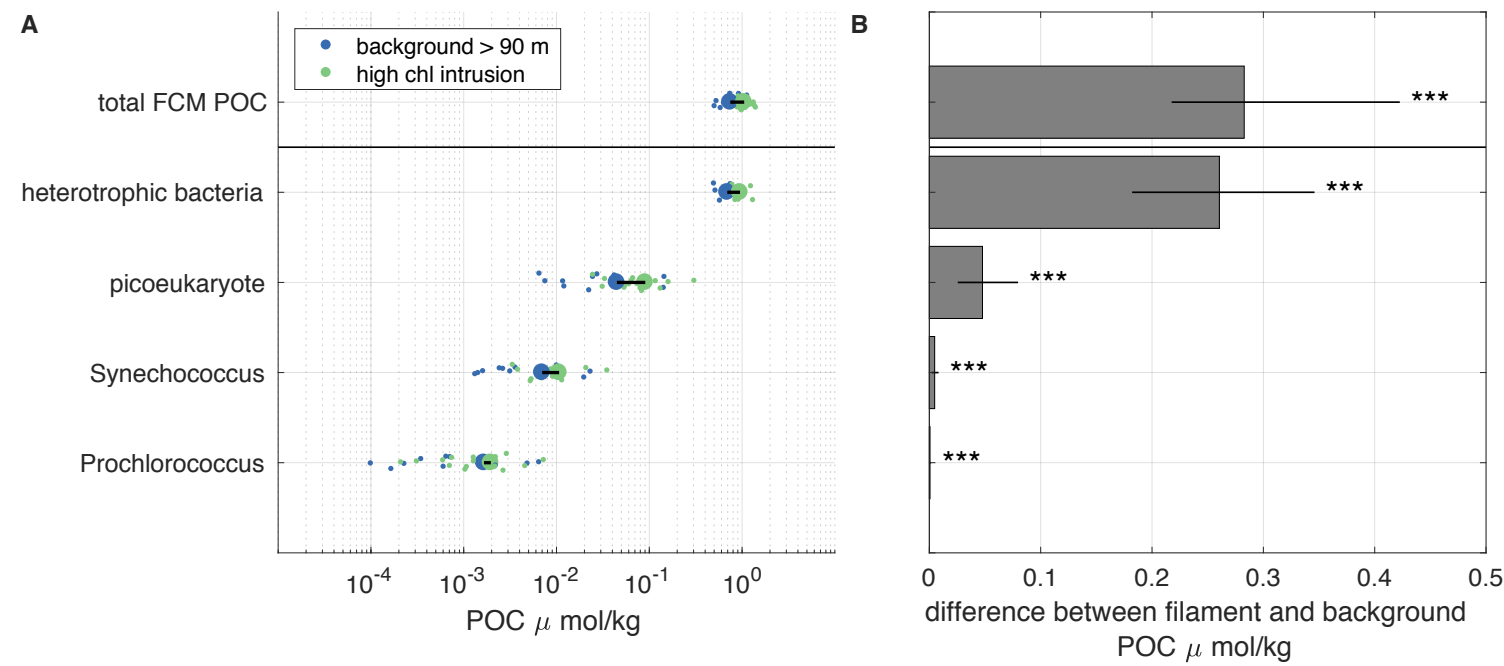

Figure 5-16: Observations of intrusions at the Western Alboran Gyre sampled during CLP19 in March and April 2019. (A) POC concentration in each taxonomic group in intrusions (green) and background samples below $100 \mathrm{~m}$ (blue). (B) Difference between the geometric mean of intrusion and background POC concentrations in each taxonomic group and the total POC concentration estimated from flow cytometry. The t-test significance level of enhancement in the intrusion over the background is shown with the stars $* * *=p<0.001$, $* *=\mathrm{p}<0.01,{ }^{*}=\mathrm{p}<0.05$.

phyll maximum will not be transported to depth through this mechanism. Communities on the light side of the front (here, in the Atlantic water) differ from the intrusion communities, forming a separate cluster in hierarchical clustering of the photosynthetic amplicons (Figure 5-13). There are two clusters that contain most of the intrusion samples, one associated with Mediterranean water intrusions (dark blue), which also contains the intrusion origin waters. The second cluster (light purple) contains the intrusion communities that subducted from Modified Atlantic water and again contains the origin waters as well. The Atlantic water chlorophyll maximum samples form a separate cluster (dark green), as do the background samples (light green, oranges). Due to heterogeneity in the origin communities, the intrusions do not converge to a unique community composition. The observation that the intrusion communities reflect the origin water mass provides additional ecological evidence of subduction and water mass origins. For example, in transects crossing the northern edge of the Western Alborán Gyre, we find high light Prochlorococcus and Synechococcus at depths of $180 \mathrm{~m}$ (Figure 5-9C2). Despite the remarkable similarity in the community composition along the intrusion with depth, the photosynthetic capacity of the cells (mea- 




Figure 5-17: Community similarity on a cross-frontal transect. The purple points show the Bray-Curtis distance from the sample at the intrusion tip at $23 \mathrm{~km}$ at the discrete sample locations. The background is AOU from the ecoCTD.

sured as $\mathrm{F}_{\mathrm{v}} / \mathrm{F}_{\mathrm{m}}$ ) declines with depth suggesting that the cells are stressed (Alou-Font et al., 2016). In another case at a Mediterranean front (transects A and C3), Prochlorococcus low light IV is only present in the DCM on the dense (Mediterranean) side of the front and in intrusion samples but not on the light (Atlantic) side of the front (Figure 5-13). Resolving succession of plankton along water mass trajectories is challenging because of limitations of sampling with existing Lagrangian instrumentation. Previous studies have been able to quantify changes along environmental gradients such as depth gradients and have observed that eddy transport can bring communities typically found at depth into the euphotic zone (Nelson et al., 2014). The dependence of exported organic matter on the origin communities has been observed in sinking fluxes as well, where the signature of the surface community is present in the exported community (Poff et al., 2021).

To illustrate the differences in community composition across the front, with depth, and within the intrusions the Bray-Curtis distance from a sample on the dense side of the front is plotted on a cross front transect (Figure 5-17). At 75-100 m, there are two distinct communities being stirred into each other. In addition, along the intrusion trajectory, the community becomes less like the origin community, suggesting that there is succession along water mass trajectories. The high resolution sampling across the front and careful attention 
to the physical features when sampling reveals that mesoscale and submesoscale processes enhance diversity through stirring communities together not just at the sea surface but also in three dimensions at depth (d'Ovidio et al., 2010; Clayton et al., 2013; Mousing et al., 2016; Tzortzis et al., 2021).

A

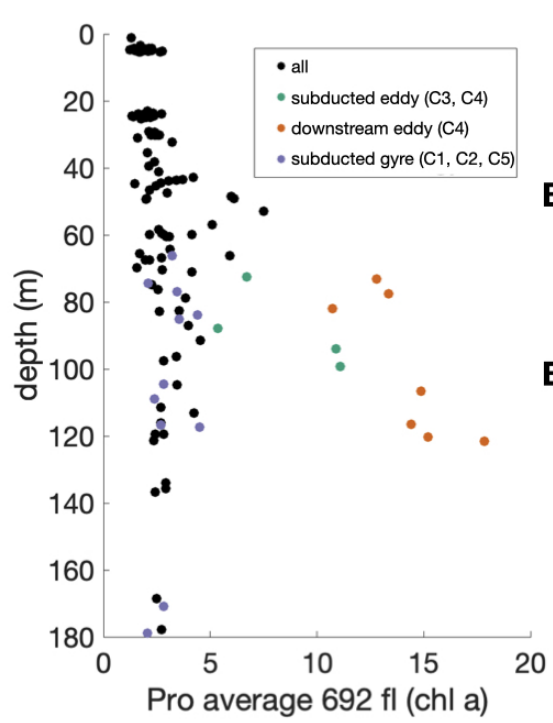

B1

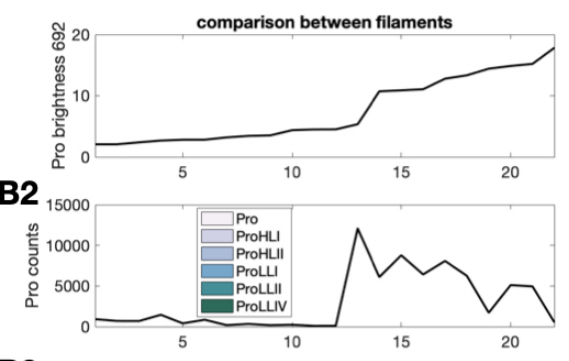

B3

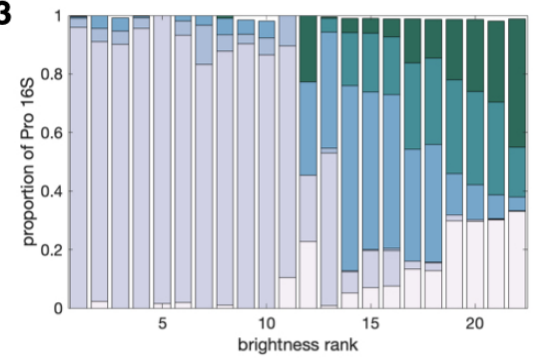

Figure 5-18: (A) Average fluorescence of Prochlorococcus cells at $692 \mathrm{~nm}$ quantified from flow cytometry. The green "subducted eddy" samples are from the intrusion on transect C3. The orange "downstream eddy" samples are from the chlorophyll maximum, intrusion, and background on transect $\mathrm{C} 4$. The purple "subducted gyre" are from intrusion samples on transects C1, C2, and C5. (B1) Average fluorescence of Prochlorococcus cells at $692 \mathrm{~nm}$ plotted in order of the average fluorescence of Prochlorococcus cells at $692 \mathrm{~nm}$ ("brightness rank"). In the other panel (B) samples, samples are plotted in the same order. (B2) Prochlorococcus cells per ml (B3) Relative proportion of Prochlorococcus 16S amplicons classified in each ecotype.

\section{Shifts in community composition}

We observe shifts in the community composition with depth in intrusions. Within a given cross-front transect of an intrusion, there is a general decreasing trend in cell counts in all groups, except for Prochlorococcus $(\mathrm{p}<0.01)$. These observations raise the possibility of in situ growth of low light Prochlorococcus strains which may still be able to grow in the low light conditions that are present in the intrusions. We will discuss the dynamics of low light Prochlorococcus further in the next section. Across all of the intrusions at Atlantic-Modified 
Atlantic water fronts, the proportion of cyanobacteria relative to picoeukaryotes increases within the intrusions relative to the intrusion origin (t-test $p=0.0023$, confidence interval $=[1.0623,1.2598]$ where 1 is no change). This could be due to either differential growth of cyanobacteria subsurface or due to a differential loss of picoeukaryotes. Previous work near the Kuroshio Current found uniform distributions of Synechococcus at depths below $200 \mathrm{~m}$, which they attributed to either differential attachment to aggregates or relatively low loss rates (Sohrin et al., 2011). The sampling along intrusions in this study demonstrates a mechanism for transport to depth that is not due to sinking and suggests that relatively low loss rates could explain the relative increase in cyanobacteria (both Prochlorococcus and Synechococcus) at depth.

Heterotrophic bacteria respond to subduction (the changing light environment) and its effects on the photosynthetic community which results in a shift in the community composition. The heterotrophic bacteria in the intrusions could be remineralizing both POC and DOC. Some of the changes in the heterotrophic bacteria community composition within the intrusions are likely due to passive mixing with the surrounding water. We used ANCOM to find statistically significant differences in the community composition between sample groups (Mandal et al., 2015). There are 16 ASVs that are detected as more abundant in the Modified Atlantic water intrusion samples than the background but not more abundant in the origin waters than in the background. These ASVs correspond to SAR406, Arctic 97B-4 marine group, MB11C04 marine group, and OCS116 clade. There are additionally 11 ASVs that are more abundant in both the Modified Atlantic Water and Mediterranean Water intrusions, which otherwise have fairly distinct community compositions both in the intrusions and in the origin waters of the intrusions. These ASVs correspond to the Flavobacteriales Cryomorphaceae and Crocinitomicaceae, the gammaproteobacteria KI89A clade, and the Verrucomicrobiales Roseibacillus. These are known to be associated with phytoplankton blooms, supporting the hypothesis that they increase in relative abundance as they remineralize the dying phytoplankton in the intrusions (Needham and Fuhrman, 2016). KI89A clade and the closely related SAR92 have been observed to increase in abundance after phytoplankton blooms and during export events in time series observations and incubations (Needham and Fuhrman, 2016; Stephens et al., 2020). OCS116 has also been observed to 
correlate with a bloom, increasing in relative abundance during eddy uplift (Nelson et al., 2014). SAR406 is typically observed in the mesopelagic and responds to environmental variability at mid-depth (Cram et al., 2015). Two of the more abundant SAR406 ASVs are more abundant in the intrusions. Across all ASVs assigned to SAR406, the relative abundance increases at the base of the euphotic zone rather than at $200 \mathrm{~m}$ (Figure 5-12).

\section{Photosynthetic growth: Prochlorococcus}

It is evident from Figure 5-14 that the relative importance of Prochlorococcus increases in the intrusion relative to the background during IRENE. This is both due to the relatively high Prochlorococcus biomass in the intrusion origin waters and an increase in the relative cell counts of Prochlorococcus with depth in the intrusion. At the edge of the anticyclonic eddy sampled during CLP19, the intrusion origin waters were also Mediterranean waters. Within the intrusion, the average brightness of Prochlorococcus increases with depth (Figure 5-18A). Although the intrusion subducted (Figure 5-9C3), we only sampled it down to $100 \mathrm{~m}$, which is within the depth range where low light Prochlorococcus ecotypes could grow. We sample the same feature in another transect $33 \mathrm{~km}$ downstream along the front and find that the same density surface of the intrusion is shallower and has brighter Prochlococcus than at the same depth upstream. The deeper samples are even brighter. By comparison, the average brightness of Prochlorococcus does not change in subducted intrusions in the WAG, where Prochlorococcus cell counts are also low (Figure 5-18B2). We attribute this increase in brightness to a shift in the Prochloroccocus community composition. Comparing the colored samples in Figure 5-18A, we see that the bright cells are associated with a higher relative abundance of the low light IV ecotype and an unclassified Prochlorococcus. Recent work has also found that the proportion of unclassified Prochlorococcus ecotypes increases with depth (Thompson et al., 2021). 


\subsection{How much does frontal subduction contribute to POC ex- port in oligotrophic gyres?}

In the Mediterranean Sea, water masses become denser due to evaporation and mixing as the current flows to the east. This means that any contributions to carbon export will in that basin will need to be considered in the context of the gyre circulation features.

The frontal dynamics and microbial communities present in the Alborán Sea are similar to those observed in other regions. Therefore, these observations provide insights about the potential for advective export in subtropical gyres. The total contribution to carbon fluxes will depend on the timescales of remineralization and shifts in the community structure relative to the timescales of the vertical motion (Chapter 3). The timescale is affected by the reversibility of the fluxes. The same water mass that is subducted will not necessarily upwell due to the mesoscale submesoscale coupling (Samelson, 1992; Freilich and Mahadevan, 2021). Freilich and Mahadevan (2021) estimated that the flux of mixed layer water into the interior is $0.25 \mathrm{~m} / \mathrm{d}$ when averaged over one month in a mesoscale domain. Assuming mixed layer POC concentrations of $6 \mathrm{mmol} \mathrm{m}^{-3}$ (Figure 5-8), the POC flux would be $1.5 \mathrm{mmol} \mathrm{m}^{-2}$ day $^{-1}\left(18 \mathrm{mg} \mathrm{C} \mathrm{m}^{-2}\right.$ day $\left.^{-1}\right)$. This modeled flux is out of the mixed layer, but not necessarily across the $100 \mathrm{~m}$ depth horizon that is often used to quantify export (Palevsky and Doney, 2018). An approximation of the POC flux across $100 \mathrm{~m}$ using the same model and evolving the average POC profile, which has the highest concentrations in the mixed layer, over one month results in an estimated $10.3 \mathrm{mg} \mathrm{C} \mathrm{m}^{-2}$ day $^{-1}$. In the stratified summer period, the maximum POC is subsurface and is co-located with a deep chlorophyll maximum. The estimated export from the deep biomass maximum in a model

representing a more stratified front has a similar magnitude of $9.9 \mathrm{mg} \mathrm{C} \mathrm{m}^{-2}$ day $^{-1}$. These estimates are performed with passive, non-reactive tracers. However, subsurface the POC concentration in a water parcel can be altered by a number of reactions including sinking, consumption, and remineralization. The POC flux may be increased if some of the POC is consumed subsurface before it is upwelled again (Chapter 3). Future work will improve the flux estimate through further modeling efforts. The estimated flux is slightly larger than the average sinking flux at $200 \mathrm{~m}$ in the Ligurian Sea (Miquel et al., 2011) and slightly smaller 
than the sinking flux at $150 \mathrm{~m}$ in the subtropical Atlantic (Owens et al., 2013). Therefore, the advective POC flux could contribute half of the total POC flux. It is possible that vertical advective fluxes may enhance the export flux captured by sediment traps so the advective and sinking export fluxes should not be considered to be completely independent (Dever et al., 2021; Fadeev et al., 2021). However, the free-living bacterial POC that forms the majority of the enhancement in POC within the observed intrusions is very slowly sinking and would likely not be captured in sediment traps. We did not measure dissolved organic carbon (DOC) here, but these same advective pathways are likely routes for DOC export as well.

\subsection{Conclusion}

Advection along sloping density surfaces at mesoscale fronts subducts organic matter from the surface ocean to the interior. The magnitude of this flux could be of similar magnitude to the sinking flux in oligotrophic regions. This advective flux occurs through a range of frontal processes, which are active throughout the year including subduction from the mixed layer and subduction from and within the stratified thermocline. This study reveals a larger than previously recognized role for heterotrophic bacteria in POC export. Advective subduction transports surface communities to depth, potentially altering the chemical composition of food supply and the microbial functioning of mesopelagic ecosystems. Our observations are concentrated in the upper $200 \mathrm{~m}$, just reaching into the upper mesopelagic. We speculate that some of the observed export could reach deeper and be sequestered, but that most is likely consumed in the water column, supporting production in the mesopelagic and deeper layers.

\subsection{Methods}

\subsubsection{Sampling strategy}

Sampling occurred during three research cruises which took place July 17-24, 2017 on the B/O Socib (IRENE), May 27-June 2, 2018 on the NRV Alliance (Calypso 2018; CLP18), 
and March 21-April 12, 2019 on the R/V Pourquoi Pas? (Calypso 2019; CLP19). To study the biological community composition during subduction, we took water samples using a CTD rosette at 6 depths. We used an adaptive sampling procedure that focused on sampling from secondary chlorophyll maxima, particularly those that corresponded to inversions in oxygen, temperature, and salinity. The cTD casts were performed at a spacing of $1-10 \mathrm{~km}$. We sampled in lines consisting of 3-8 CTD casts in a cross-front transect. The transects are oriented across the front to capture the greatest degree of variability in water masses and isopycnal depth range. Each transect was completed as rapidly as possible with 30 minutes to 1 hour between casts on a given transect. In 2018 and 2019, we also arranged transects in upstream and downstream pairs to study the along-front variability in addition to the crossfront variability. CTD cast locations were chosen based on cross-front uCTD or ecoCTD transects performed in the same cross-front section immediately prior to the CTD transect. Water samples were picked from CTD casts based on an adaptive sampling procedure. Samples were collected using Niskin bottles mounted to a CTD rosette. On every cast, samples were collected at

- Surface (5m - fixed)

- Mixed layer ( $\sim 25$ meters)

- Deep chlorophyll maximum or lower mixed layer

- Lower deep chlorophyll maximum

- Secondary chlorophyll maximum or other intrusion. If not present, fixed depth at 120 meters.

- The maximum cast depth (200 $\mathrm{m}$ in $2017,250 \mathrm{~m}$ in 2018)

Transects of surface only samples were taken in 2017 and 2018. Water samples were collected on these transects with an oceanographic bucket with the ship traveling at 8 knots. The samples were processed using the same methods as the CTD casts. 


\subsubsection{Temperature and salinity}

Temperature and salinity were measured with a Seabird SBE9 on the CTD rosette and with two different underway profilers the underway CTD (Oceansciences) and the EcoCTD. The EcoCTD measures conductivity, temperature, and pressure with an RBR Concerto CTD (Dever et al., 2020). The data processing from the underway instruments is described in Dever et al. (2019). Discrete samples were taken to calibrate the SBE9 and the EcoCTD and $\mathrm{uCTD}$ were calibrated by mounting them to the CTD rosette frame for one cast.

\subsubsection{Nutrients}

A SUNA V2 nitrate sensor was mounted to the CTD frame. Samples for inorganic nutrient concentrations were taken at each sample depth. Samples were frozen at $-20^{\circ} \mathrm{C}$ and analyzed to determined the concentrations of nitrate, nitrite, silicate, and phosphate with a nutrient autoanalyzer at the Institute for Marine Sciences of Andalusia (ICMAN-CSIC).

\subsubsection{Chlorophyll}

Chlorophyll fluorescence was measured by a Chelsea Aqua 3 fluorometer (CLP18) and WET Labs ECO-AFL/FL (CLP19) mounted on the CTD and a Seabird ECO Puck on the EcoCTD. In vivo fluorescence was calibrated with in situ samples for total chlorophyll a (chl a) concentration. Samples were collected from the fluorescence maximum and at the maximum profile depth (200 or $250 \mathrm{~m}$ ). Each sample consisted of $0.5 \mathrm{~L}$ of seawater filtered onboard through a $45 \mathrm{~mm}$ GF/F Whatman filter. At the laboratory on shore, samples were extracted for 24 hours in $90 \%$ acetone in the dark for fluorometric determination (Turner Designs, trilogy fluorometer).

\subsubsection{Oxygen}

Oxygen was measured by a Seabird SBE43 oxygen sensor mounted to the CTD and a RINKO oxygen sensor on the EcoCTD. The output of the oxygen sensors was calibrated against the Winkler titrations performed at sea within 48 hours of sampling (Metrohm 888 Titrator). 


\subsubsection{Photosystem II efficiency $\left(\mathrm{F}_{\mathrm{v}} / \mathrm{F}_{\mathrm{m}}\right)$}

The photosynthetic performance of algae was assessed by examining the changes in chlorophyll fluorescence with the electron transport inhibitor 3.(3,4-dichlorophenyl)-1,1-dimethylurea (DCMU) that blocks electron transport at the electron acceptor Q in PSII. Minimum and maximum fluorescence were measured after 30 min of dark adaptation using a Turner Designs 10-AU fluorometer on board.

\subsubsection{Respiration rate}

To better interpret changes in AOU along- and across-fronts, we measured the biochemical oxygen demand (BOD) in a subset of the samples in 2019 using the autoBOD, a device that measures oxygen continuously using an oxygen optode Collins et al. (2018). We sampled BOD at a maximum of two depths in each CTD cast. The samples were taken below the euphotic depth and within and outside of subducted filaments (AOU anomalies). We also measured the BOD at one sample in the inferred source waters of a subducted filament (this sample may be from the euphotic zone, but was incubated in the dark). We took 4 replicates of each sample from the same Niskin bottle. Incubations took place at $14^{\circ} \mathrm{C}$ in the dark in the temperature controlled room of the Pourquoi Pas?.

\subsubsection{Biological community composition}

\section{DNA Extraction, PCR, and Sequencing}

DNA samples for characterizing microbial communities were obtained by filtering biomass from $500 \mathrm{ml}$ of natural seawater onto $47 \mathrm{~mm} 0.2 \mu \mathrm{m}$ pore size polyethersulfone membrane filters (Supor 200, Pall Gelman). Filters were placed into sterile cryovials, flash-frozen in liquid nitrogen and transferred to $-80^{\circ} \mathrm{C}$ until extraction using the DNeasy Plant Kit (Qiagen), with a modification including a bead beating step Demir-Hilton et al. (2011). During the 2017 and 2018 cruises, samples were stored in liquid nitrogen for the duration of the cruise after which they were stored at $-80^{\circ} \mathrm{C}$ until extraction. DNA was amplified using the primers $27 \mathrm{FB}\left(5^{\prime}-\right.$ AGRGTTYGATYMTGGCTCAG-3') and 338RPL (5'-GCWGCCWCCCGTAGGWGT-3') as in Vergin et al. (2013b); Sudek et al. (2015) targeting the V1-V2 hypervariable region of 
the $16 \mathrm{~S}$ rRNA gene with Illumina adapters, allowing us to explore the diversity of eukaryotic phytoplankton (via plastid 16S), cyanobacteria, and heterotrophic bacterioplankton Vergin et al. (2013b); Sudek et al. (2015); Choi et al. (2017, 2020). PCR reactions contained $25 \mathrm{ng}$ of template, $5 \mu \mathrm{l}$ of $10 \times$ buffer, $1 \mathrm{U}$ of HiFi-Taq, $1.6 \mathrm{mM} \mathrm{MgSO} 4$ (Thermo Fisher) and 0.2 $\mu \mathrm{M}$ of each primer. The PCR cycling parameters were $94^{\circ} \mathrm{C}$ for $2 \mathrm{~min} ; 30 \times 94^{\circ} \mathrm{C}$ for $15 \mathrm{~s}$, $55^{\circ} \mathrm{C}$ for $30 \mathrm{~s}, 68^{\circ} \mathrm{C}$ for $1 \mathrm{~min}$, and a final elongation at $68^{\circ} \mathrm{C}$ for $7 \mathrm{~min}$. Paired-end library sequencing $(2 \times 300 \mathrm{bp})$ was performed using the Illumina MiSeq platform (Illumina).

\section{$16 \mathrm{~S}$ rRNA amplicon analyses}

Sequences were demultiplexed and assigned to corresponding samples using CASAVA (Illumina). A 10 bp running window was utilized to trim low-quality sequence ends at a Phred quality (Q) of 25 using Sickle 1.33 Joshi and Fass (2011). Paired-end reads were merged using USEARCH v10.0.240 Edgar and Flyvbjerg (2015) when reads had a $\geq 50$ bp overlap with maximum $5 \%$ mismatch. The merged reads were then filtered to remove reads with maximum error rate $>0.001$ or shorter than $200 \mathrm{bp}$. Only sequences with exact match to both primers were kept and primer sequences were trimmed using Cutadapt v.1.13 Martin (2011). After removal of single sequence reads, the average number of amplicon reads from the 317 samples was $259,104 \pm 133,685$. Cyanobacterial and plastid amplicons were initially parsed using the phylogenetic pipeline in PhyloAssigner v.6.166 Vergin et al. (2013b). Amplicons from plastids and cyanobacteria were further classified using a global plastid and cyanobacterial reference alignment and tree according to protocols outlined in Choi et al. (2017). Cyanobacterial amplicons were analyzed using fine-scale cyanobacterial reference alignment and tree Sudek et al. (2015), and plastid amplicons were further analyzed using multiple high-resolution alignments and trees focusing on stramenopiles and viridiplantae Choi et al. (2017, 2020). For the assessment of heterotrophic bacterioplankton communities, sequences (82,135,874 reads) were resolved into 49,150 amplicon sequence variants (ASVs) of 100\% pairwise identity with USEARCH v10.0.240 Edgar and Flyvbjerg (2015). Taxonomies were assigned to each ASV using classify-sklearn by QIIME2 Bokulich et al. (2018) searching against the SILVA database release 138 Quast et al. (2012). 


\section{Cell quantification}

We preserved samples for quantification by flow cytometry from the same Niskin bottles from which we took the samples for DNA extraction. Samples were preserved using $10 \mu \mathrm{l}$ of EM grade 25\% Glutaraldehyde per $1 \mathrm{ml}$ seawater and then flash frozen in liquid nitrogen (or at $-80^{\circ}$ for casts $1-19$ in 2019 ). Samples were then stored at $-80^{\circ} \mathrm{C}$ until analysis. Cells were quantified using a BD Influx flow cytometer equipped with a $488 \mathrm{~nm}$ laser. Forward angle light scatter, side scatter, and autofluorescence at $692( \pm 40) \mathrm{nm}, 572( \pm 27) \mathrm{nm}$, and 520 $( \pm 25) \mathrm{nm}$ were recorded. Photosynthetic cells were counted by triggering on forward angle light scatter. Calibration beads were added to each sample immediately before analysis (0.75 $\mu \mathrm{m}$ yellow-green, Polysciences, Inc and 1.0-1.4 $\mu \mathrm{m}$ ultrarainbow, Spherotech). Each sample was run for 8 minutes at $25 \mu l \mathrm{~min}^{-1}$ after a pre-run of 2 minutes. Heterotrophic bacteria cells were counted by staining samples with SYBR Green and triggering on autofluorescence at $520 \mathrm{~nm}$. Samples were run for 4 minutes after a 2 minute pre-run.

\subsubsection{Statistical analyses}

Beta diversity is computed using ordination in phyloseq McMurdie and Holmes (2013). Ordination aids interpretation of the dissimilarity between the samples (Bray-Curtis dissimilarity). We present the results of non-metric multidimensional scaling (NMDS), which is an iterative, non-parametric ordination technique.

We test which ASVs have statistically significant different abundances (differentially abundant) between sample groups using ANCOM implemented in Qiime2. We define three groups for this analysis, the chlorophyll maximum on the dense side of the front and intrusion samples shallower than $90 \mathrm{~m}$ (origin waters), the samples in the intrusions, and samples in the background deeper than $90 \mathrm{~m}$. ANCOM assumes that fewer than $25 \%$ of the ASVs change between groups (Mandal et al., 2015).

\subsubsection{Computing particulate organic carbon (POC)}

We compute the POC concentration using multiple complimentary methods. Each captures a different contribution to the POC. 


\section{Flow cytometry}

We use literature values Worden et al. (2004) and Buitenhuis et al. (2012). The carbon per cell is most uncertain for the picoeukaryotes, which have the largest biomass per cell and are a large contributor to the small photosynthetic community during the 2019 cruise. Ducklow and Harris (1993) use a value of $20 \mathrm{fg} \mathrm{C}^{-1} \mathrm{cell}^{-1}$ for heterotrophic bacteria (Lee and Fuhrman, 1987) (Figure 5-11).

\section{Optical instruments}

The particle beam attenuation is a proxy for particles in the size range 5-20 $\mu \mathrm{m}$ Stramski et al. (1995). The beam transmission (Tr) (Seatech/Chelsea in 2018, Wetlabs in 2019) was measured and used to derive $c_{p}$ as

$$
c_{p}=\frac{1}{0.25} \ln \frac{\operatorname{Tr}}{100}
$$

The community composition, in addition to the total POC, may affect $c_{p}$. The beam attenuation is affected by phytoplankton and also by bacteria, zooplankton, and detritus. It is not currently possible to measure beam transmission on the EcoCTD. Instead, the EcoCTD measures backscatter at $700 \mathrm{~nm}$. At calibration casts where the EcoCTD is attached to the ship-board CTD frame, we find a linear correlation between $c_{p}$ and $b_{b p}(700)$ (Figure E-2). In the northwestern Mediterranean, the diel cycles of $c_{p}$ and $b_{b p}(700)$ are not well correlated indicating that there are differential contributions of phytoplankton to these proxies Kheireddine and Antoine (2014). $c_{p}$ shows larger diel variations Gernez et al. (2011) suggesting that small detritus and bacteria make larger contributions to $b_{b p}$ than to $c_{p}$.

Both the beam attenuation and backscatter can be converted to carbon using region-specific empirical calibrations. We use a correlation between $c_{p}$ and POC of $1.78 \mathrm{~m}^{2}(\mathrm{~g} \mathrm{C})^{-1}$ Oubelkheir et al. (2005) (Figure 5-10). The range of possible values includes the values reported in Rasse et al. (2017). We use a relationship between backscatter and POC from Mayot et al. $(2017)\left(\mathrm{POC}=54463 \times b_{b p}-1.19 \mathrm{mg} \mathrm{C} \mathrm{m}^{-3}\right)($ Figure E-1). 


\subsubsection{Computing POC flux}

The POC flux is computed using process study models initialized with a cross section across the Almería-Oran front. The two models represent the stratified period (when the initial condition was sampled) and the springtime period when the thermocline density surface outcrop at the sea surface (Freilich and Mahadevan, 2021). The initial condition representing the spring time period is obtained by cooling the sea surface and using convective adjustment until the mixed layer has a maximum depth of $70 \mathrm{~m}$. The POC flux is computed by evolving the mean POC profile from each season for a month with no reactions or restoring. The total POC flux is estimated as the average carbon concentration integrated between the model base and $90 \mathrm{~m}$. 


\section{Chapter 6}

\section{Conclusions}

In the present moment, among the most compelling reasons for studying open ocean biophysical interactions is climate change. Half of the global primary production takes place in the oceans and the biological carbon pump sequesters atmospheric, anthropogenic carbon in the ocean. Global climate models are low resolution and do not represent submesoscale dynamics, leading to structural uncertainty in predictions in proportion to the influence of submesoscale dynamics on the relevant biogeochemical processes (Uchida et al., 2020). There are major uncertainties about the magnitude of the biological carbon pump and the ways the climate change will affect ocean ecosystems (Henson et al., 2011; Siegel et al., 2014). Fundamental understanding may help to reduce uncertainty and provide sound basis for decision-making that relies on information about primary production. Due to the context of climate change, this dissertation focuses on biogeochemical tracers that are relevant for climate including carbon and primary production. The hypothesis that fundamental understanding may decrease uncertainty about future conditions informs our approach to the research.

In this dissertation, I study upper ocean dynamics from a Lagrangian perspective. I focus on aspects of the physics of the vertical motion that have the potential to illuminate the impacts of ocean circulation on biogeochemistry. We find that it is necessary to consider the pathways and timescales of vertical motion to explain how coupling between vertical velocity and biological processes determines nutrient and carbon fluxes in the upper ocean. Ocean primary production and carbon export is not uniformly distributed but instead is 
shaped by light availability and nutrient supply (and other ecological processes). Even within the nutrient-limited subtropical gyres, primary production takes place in variable openocean environments provided by fronts and eddies where the vertical velocity magnitude is enhanced. The vertical velocity associated with these features transports material, including but not limited to biogeochemical materials, between the surface and interior and across the mixed layer base. Strong vertical velocity at the submesoscale have small spatial scales but are also rapidly evolving in time. Vertical transport is determined by the way that water parcels transit through this time evolving flow field and is shaped by both the vertical velocity and the horizontal velocity, which remains orders of magnitude larger than the vertical velocity. The impact of transport pathways on the global carbon cycle depends on the ways that these pathways interact with nutrient distributions and ecological communities to contribute to biogeochemical fluxes. In this dissertation we ask

I. What are the characteristics of submesoscale vertical tracer transport?

II. How does phytoplankton physiology affect nutrient uptake?

III. How is water transported across the base of the mixed layer?

IV. What is the contribution of frontal subduction to carbon export in subtropical regions?

Below I outline some of the synthetic insights gained from addressing these questions and outline some directions for future research.

Connections between mesoscale and submesoscale. Observations of ocean currents contain the signatures of a wide range of different types of motion including waves, mesoscale and submesoscale instabilities, and convection, which can be difficult to separate. Vertical velocity has a range of scales and has more power at small spatial scales than horizontal velocity does. Although there is continuity between the mesoscale and submesoscale, we determine that the characteristics of the vertical velocity differ at the small scale in ways that are related to the non-linear nature of submesoscale frontal dynamics. In chapter 2 we demonstrate that at small spatial scales, the vertical velocity transitions from being mostly related to the heaving of isopycnal surfaces to an equal contribution from vertical motion along sloping isopycnal surfaces. This result has particular implications for tracer fluxes 
because unlike buoyancy, vertical transport of biogeochemical tracers can occur both due to the movement of isopycnals and due to motion along sloping isopycnal. This vertical velocity decomposition can only be carried out below the mixed layer but exchange across the mixed layer base is crucial for exchange between the surface and the interior.

Mesoscale fronts are deep reaching, increasing their potential for biogeochemical exchanges (Mahadevan, 2016; Lévy et al., 2018) and submeoscale dynamics are enhanced in the ocean surface, increasing their potential for driving exchange near the sea surface. In chapter 5 we analyze observations from the Western Mediterannean Sea that demonstrate that subduction and upwelling occurs at mesoscale fronts and due to submesoscale features on those fronts. In chapter 4 we design and analyze a process study model based on these observations. We find that subduction from the mixed layer to the interior occurs due to frontogenesis at the mesoscale front. This frontogenesis is related to the submesoscale along front variability and generated coherent subduction at scales of $\sim 10 \mathrm{~km}$. There is no single process involved in subduction, but rather a range of geostrophic and ageostrophic processes. While gaining this understanding from the process study model and observations advances our understanding of coupling between the mesoscale and submesoscale and the wide range of dynamics, this chapter also reveals limitations of our current understanding of frontal dynamics and submesoscale processes. In situations such as this one where material transport occurs as a result of multiple interacting non-linear processes there are physical dynamics that remain to be fully understood.

The connection between the mesoscale and submesoscale has practical implications for climate and ocean modeling. In chapter 2 we take a traditional approach to separating the mesoscale and submesoscale by comparing low and high resolution models. Low resolution models have weaker vertical velocity and the unresolved vertical velocity is disproportionately along sloping isopycnal surfaces. We then demonstrate that by changing the model density structure (increasing the large scale isopycnal slope), we obtain variations in the proportion of the vertical velocity that is along sloping isopycnal surfaces that fall on the same scaling relationship as the variations due to changing model resolution. In this way, we are able to point to the dynamics that differ between mesoscale and submesoscale in a systematic way. This approach introduces a new method for examining the differences between 
mesoscale and submesoscale in process study models by exploring the parameter space of the hydrographic structure in addition to the model resolution. The scaling relationship derived in chapter 3 could be used to make progress towards improve paramterization of along isopycnal fluxes, particularly of biogeochemical tracers for which the vertical transport is essential.

All of the process study models in this dissertation are carried out with no wind forcing and only weak surface buoyancy forcing. Mesoscale-submesoscale coupling could be altered in the presence of wind or buoyancy forcing due to the interactions between fronts and winds or mixed layer processes and surface forcing. Wind forcing would result in a larger wave field, which could alter the nonlinear vertical velocity and vertical transport dynamics. This is one avenue for future research.

The temporal scale of vertical motion is related to the spatial scale, with the expectation that there will be higher frequency vertical motion when the vertical velocity has more small spatial scale variability. However, the interaction between the mesoscale and submesoscale may alter the apparent temporal scale of vertical velocity. When analyzed in an Eulerian frame, the vertical velocity wavenumber-frequency spectrum of a model used in chapters 2 and 3 has a fairly rapid non-dispersive wavenumber-frequency relationship (Appendix B), potentially suggesting coupling between mesoscale and submesoscale dynamics. There is continuity between the mesoscale and submesoscale in this model. The lessons about continuity and change in dynamics with spatial scale likely also apply to the temporal scale.

Lagrangian pathways. We address the issue of the temporal dynamics of vertical velocity in more detail using Lagrangian methods. Lagrangian analysis allows us to track water parcels as they transit through the flow field, revealing the pathways for transport, change in dynamics along trajectories, fluctuations in light due to change in depth, and other aspects of the dynamics. In each of the chapters we use Lagrangian analysis. ${ }^{1}$ One of the reasons for this is that the horizontal velocity is orders of magnitude faster than the vertical velocity and isolating the vertical velocity processes requires selecting a Lagrangian frame (e.g. the rotational velocity field in chapter 2). Another reason is that Lagrangian

\footnotetext{
${ }^{1}$ I first learned about Lagrangian analysis as a summer student fellow at WHOI where I worked on Lagrangian analysis in the Bay of Bengal and contributed to Mahadevan et al. (2016)
} 
analysis allows us to disentangle mechanisms of biophysical coupling by performing analysis in the frame of the dynamics experienced by plankton. The Lagrangian analyses in this dissertation suggest that fluxes should be conceptualized in ways that go beyond purely diffusive fluxes in order to account for non-local processes including the spatial coherence of subduction locations and the ways in which biological and physical timescales couple to alter biogeochemical tracer anomalies.

Using Lagrangian analysis in chapter 4 we determine that water parcels leave the mixed layer in spatially localized patches at the mesoscale front that are variable in time. We also learn that despite the presence of $20 \mathrm{~m}$ /day vertical velocity in the mixed layer, the average rate at which a water parcel transitions across the mixed layer base is $5 \mathrm{~m}$ /day and that subduction occurs on timescales of 3 days. We also use Lagrangian tools to examine the evolution of dynamics along trajectories and find consistent patterns in the evolution prior to subduction that suggest important roles for frontogenesis and non-linear dynamics.

The influence of frontal dynamics on biogeochemical cycles depends on both the pathways of vertical transport and on the ways that diverse microbial communities respond to the enhanced vertical motion at fronts. Using Lagrangian analysis in chapter 3, we find that the relative rate of biological uptake and vertical transport determines the overall biogeochemical flux. This means that the time history of water parcels has implications for the fluxes. The non-linearity of fluxes even with linear biological reactions is particularly striking and explained using theoretical analysis along Lagrangian trajectories. This finding also has implications for biogeography; regions with different vertical velocity characteristics could support very different phytoplankton communities. This analysis is also revealing about the temporal dynamics of vertical motion. Despite a red vertical velocity frequency spectrum, a nutrient uptake timescale of 3 days, which is a submesoscale-like timescale, maximizes the nutrient fluxes. From the perspective of modeling, when we include additional physical processes with faster temporal scales and smaller spatial scales, we find that they can affect the biogeochemical outcomes due to the relative timescale of biological processes, which is poorly constrained and often held fixed, and the physical timescales.

Effects of submesoscale physics on primary production. Submesoscale dynamics affect the rate of primary production in subtropical gyres by enhancing nutrient supply 
(Mahadevan, 2016). Chapters 2 and 3 explain two important ways in which submesoscale dynamics are particularly important for primary production in subtropical oligotrophic gyres. Along isopycnal vertical transport supplies nutrients to the euphotic zone in the presence of fronts and eddies. In addition, the timescales of submesoscale dynamics are similar to the observed biological timescales, leading to increased nutrient fluxes.

The observational and modeling studies in this dissertation were carried out at the submesoscale and mesoscale. In some cases, processes that are locally important may not be globally influential. The global importance of submesoscale dynamics for biogeochemical fluxes is still a matter of debate. The upcoming surface water and ocean topography (SWOT) and Plankton, Aerosol, Cloud, ocean Ecosystem (PACE) satellite missions will provide global observations of surface currents at higher spatial resolution and phytoplankton communities at higher taxonomic resolution than currently available observations. SWOT will measure sea surface height with more accuracy than current satellites, which can be used to derive the geostrophic currents at the small end of the mesoscale range. PACE will measure ocean color at finer wavelength resolution than past instrumentation which can be used to map the distribution of phytoplankton pigment types. SWOT will not directly measure the vertical velocity but coupling of observations from SWOT and PACE may reveal new insights about the dynamics and consequences of physical transport for marine ecology and biogeochemistry.

In addition to altering productivity, submesoscale dynamics can also alter the community composition (d'Ovidio et al., 2010; Clayton et al., 2014; Lévy et al., 2015). The dataset analyzed in chapter 5 also contains evidence of upwelling and modification of community composition on fine spatial scales. This dataset can be analyzed to provide evidence of the influence of submesoscale vertical velocity in modifying community composition. Previous work has found evidence for the influence of stirring (d'Ovidio et al., 2010; Clayton et al., 2014; Mousing et al., 2016) but observations of the effects of vertical velocities is more limited (Rodriguez et al., 2001).

Effects of submesoscale physics on carbon export. While the first half of this dissertation focuses on upward transport of nutrients, the second focuses on downward transport (subduction). Subduction has implications for biogeochemical properties that are 
exchanged at the sea surface such as carbon and oxygen. Here we focus on carbon export.

The observations in the Western Mediterranean Sea analyzed in chapter 5 suggest that vertical motion along isopycnal surfaces is a pathway of carbon export from the surface to the interior in subtropical regions. Subduction of biomass at fronts and eddies provides a pathway for the transport of small, non-sinking POC from the surface to the interior. In subtropical gyres, more of the biomass is in small size fractions compared with more nutrientreplete ocean biomes. The relative importance of subduction compared with sinking to the carbon export increases when the relative biomass in the small size fractions is larger (Dever et al., 2021). The rate of advective export is estimated by combining the observations with the Lagrangian and tracer analysis of the model analyzed in chapter 4. Just as the realization that mesoscale eddies exert a control on the rate of primary production changed the way that we understand the controls on ocean ecosystems, the realization that subduction of biomass makes a quantitatively important contribution to carbon export should prompt a reconsideration of the ecological and biogeochemical role of primary production in oligotrophic gyres. An immediate next step is to improve the quantification of how much of the production in a oligotrophic region is exported due to advective fluxes rather than recycled within the euphotic zone. This process is likely important in other subtropical gyres. Preliminary analysis reveals that the carbon intrusions studied here likely also appear at the Bermuda Atlantic Time Series. A more detailed analysis will be carried out to study the occurrence of these features from point measurements rather than spatial surveys, which will help inform other studies that do not perform the same spatially-intensive sampling that was carried out for this dissertation. The advective export pathway provides a source of carbon to the mesopelagic and affects the distribution of microbial organisms within the mesopelagic. Because the rates of organic matter transformation in the mesopelagic are affected by the microbial community, the rate of remineralization of both subducted and sinking organic matter may be affected by the frequency and subduction of surface communities.

The advectively-driven POC flux identified here is expected to be a function of depth. Previous theories such as the Martin curve focus on the sinking flux (Martin et al., 1987; Cael and Bisson, 2018). Omand et al. (2015) provides a physically-driven parameterization for mixed layer restratification. Here we look at flux beneath the mixed layer and along 
isopycnal across the mixed layer base. We assume in these cases that the export is mostly along isopycnals. The question of how to parameterize this POC flux is not yet resolved. One approach would be model this flux as a diffusive flux along isopycnal Redi (1982). Much of the literature on cross-frontal exchange has been carried out within the framework on diffusive fluxes. However, even for fluxes of temperature and salinity the diffusive framework has some limitations (Chapters 2 and 4). There are additional limitations in the context of biogeochemical fluxes due to coupling between the biological and physical processes. Patchy productivity can produce a covariance between vertical velocities and carbon anomalies which will not be represented by the symmetric diffusivity tensor. The results from this thesis could inform parameterization of along isopycnal biogeochemical fluxes. A parameterization could be used to diagnose carbon export in both models and observations that do not resolve mesoscale and submesoscale fluxes.

Interdisciplinary oceanography. I would like to close with a note about the methods. The work in this dissertation combines theory, modeling, and observations. Observations are guided by theory, modeling is grounded in observations, models animate limited observations, theory is tested and challenged in models, and modeling is generalized by theory.

In many cases, it can be difficult to observe and model the same parameters. Observations include many more processes and factors than can be reasonably constrained in a model. On the other hand, observations of some of the key quantities - like vertical velocity - are not available. Models complement the observations. In each process study model used in this dissertation, observations influence the way that the set up the problem and the parameter space that we explore. In this way, the models are idealized and grounded in observations. While this approach allows us to grapple with environmental complexity, we risk extrapolating our models and theoretical results to regions where they do not apply. For example, while the application of the scaling relationship in chapter 2 to a global model is intriguing, it should not be viewed as a definitive distribution of vertical velocity characteristics. The scaling relationship was only tested with parameters from the mid-latitudes, specifically the North Atlantic subtropical gyre and the high values in the tropics where the Coriolis parameter is small and high latitudes where the mixed layer is deep should be taken as an invitation to study this process further in those regions. Even when attempting 
to compare across potentially similar regions of the world ocean, the uneven distribution of global sampling means there is the risk of extrapolating observations from well-studied regions to poorly sampled regions where the processes studied are not as relevant. Future work, and particularly global parameterization, should more carefully consider application to regions outside the relatively well-sampled northern hemisphere subtropical gyres.

This dissertation forges new pathways in interdisciplinary oceanography. Biological sampling at the submesoscale has revealed coupling between biological and physical processes. At the same time, the consideration of biological phenomena points to unresolved questions about ocean physics. 


\section{Appendix A}

\section{Derivations of flux dependence on biological timescale and model assumptions}

\section{A.1 Biological model and discussion of assumptions}

The biological model used in the main text can be derived from a nutrient $(N)$ - phytoplankton $(P)$ model. We model the evolution of the phytoplankton concentration as

$$
\frac{\mathrm{d} P(t ; z)}{\mathrm{d} t}=r(z) N(t ; z) P(t ; z)-m(z) P(t ; z) .
$$

In this model, the growth rate $r$ and mortality/respiration rate $m$ are both functions of depth. This is a non-linear model that is related to the commonly-used logistic growth equation. We can linearize this model and, assuming a conservative nutrient and phytoplankton system with a depth-dependent total concentration $N_{T}$ that is partitioned between the nutrient and phytoplankton pools such that $N_{T}=N+P$ write it in terms of nutrients rather than phytoplankton to obtain the model used in the main text

$$
\frac{\mathrm{d} N}{\mathrm{~d} t}=-\lambda\left(N-N_{0}(z)\right)
$$



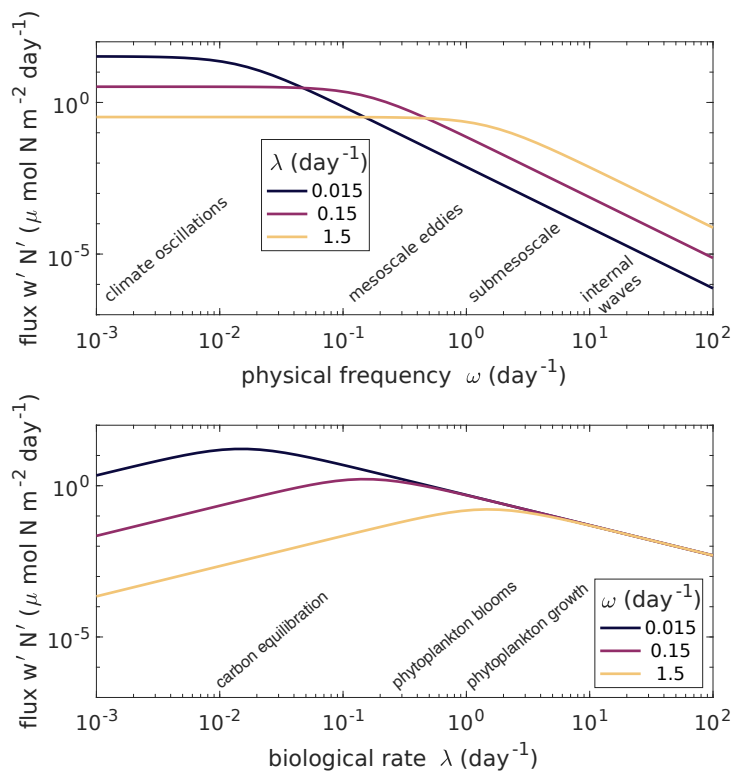

Figure A-1: (top) Vertical nutrient flux as a function of growth rate for a range of vertical velocity frequencies. (bottom) Vertical nutrient flux as a function of vertical velocity frequency for a range of growth rates. The vertical flux is computed using the idealized model in equation A.16.

In this model, the parameters $\lambda$ and $N_{0}(z)$ are both combinations of $r, m$, and $N_{T}$. The biological rate $\lambda$ is the growth rate in the logistic formulation of the model. We choose to prescribe $\lambda$ as constant and include all depth dependence in $N_{0}(z)$. This is possible by prescribing a relationship between $r(z), m(z)$, and $N_{T}(z)$ such that only two of those three variables can be assigned independently. The full non-linear form has complex behavior that captures phenomena such as blooms, which are not represented with a linearized growth function. Using this linearized model, the general equation for the evolution of the nutrient concentration is

$$
\frac{\partial N}{\partial t}+\nabla \cdot(\mathbf{u} N)=-\lambda\left(N-N_{0}(z)\right)
$$

The major assumption in this equation is that the biological function can be appropriately modeled as a linear approximation. This assumption may be violated in certain circumstances. 


\section{A.2 General theory}

Since the growth rate in equation 1 only depends on the vertical position, we can isolate the terms that depend on the vertical coordinate and focus on these when analyzing the vertical nutrient flux.

$$
\frac{\partial N}{\partial t}+w \frac{\partial N}{\partial z}=-\lambda\left(N-N_{0}(z)\right)
$$

Decomposing the nutrient concentration into a background concentration $\left(N_{0}\right)$ and an anomaly $\left(N^{\prime}\right), N=N_{0}(z)+N^{\prime}$, we can derive an equation for the evolution of the nutrient anomaly

$$
\frac{\partial N^{\prime}}{\partial t}+w\left(\frac{\partial N_{0}(z)}{\partial z}+\frac{\partial N^{\prime}}{\partial z}\right)=-\lambda N^{\prime}
$$

We make the simplifying assumption that the vertical velocity magnitude is uniform with $z$ and that $N_{0}(z)$ is a linear function of $z$ within the average fluctuation length scale of a water parcel. The combination of this assumption with the assumption that $\lambda$ is constant with depth means that we can assume that $N^{\prime}$ is a linear function of depth. Therefore, we can write $\frac{\partial N}{\partial z}=\gamma$ and derive the simplified equation A.6

$$
\frac{\partial N^{\prime}}{\partial t}+\gamma w=-\lambda N^{\prime}
$$

We solve for the nutrient anomaly using the integrating factor method

$$
N^{\prime}=C_{0} \int_{0}^{t} e^{-\lambda(t-s)} w(s) d s+C_{1}
$$

The vertical nutrient flux averaged over the interval $[0, t]$ is then

$$
\overline{w^{\prime} N^{\prime}}=C_{0} \int_{t_{0}}^{t} e^{-\lambda(t-s)} w(s) w(t) d s+C_{2}
$$

\section{A.3 Simplified physics}

To build intuition for the dependence of the vertical nutrient flux on the interaction between biological and physical timescales, we examine a case in which the vertical velocity oscillates 
symmetrically with a frequency given by $\omega$ and an amplitude given by $a$.

\section{A.3.1 Flux}

The time evolution of the nutrient concentration $N$ is given given by equation A.8.

$$
\frac{\partial N}{\partial t}+a \sin (\omega t) \frac{\partial N}{\partial z}=-\lambda\left(N-N_{0}(z)\right)
$$

The flux is defined as the time-averaged covariance between nutrient anomaly and vertical velocity anomaly (equation A.10)

$$
\text { flux } \equiv\left\langle w^{\prime} N^{\prime}\right\rangle
$$

We can solve for the vertical velocity anomaly using the relationship $N=N_{0}(z)+N^{\prime}$

$$
\frac{\partial N^{\prime}}{\partial t}+a \sin (\omega t)\left(\frac{\mathrm{d} N_{0}}{\mathrm{~d} z}+\frac{\partial N^{\prime}}{\partial z}\right)=-\lambda N^{\prime}
$$

by making the Ansatz $N^{\prime}=A \sin (\omega t)+B \cos (\omega t)$.

$$
A \omega \cos (\omega t)-B \omega \sin (\omega t)+a \frac{\mathrm{d} N_{0}}{\mathrm{~d} z} \sin (\omega t)=-\lambda A \sin (\omega t)-\lambda B \cos (\omega t)
$$

collecting the terms

$$
\begin{array}{r}
A \omega+\lambda B=0 \\
-B \omega+a \frac{\mathrm{d} N_{0}}{\mathrm{~d} z}+\lambda A=0
\end{array}
$$

solving for $A$ and $B$ and substituting into the equation for the nutrient anomaly $N^{\prime}$

$$
N^{\prime}=\frac{-\lambda a \frac{\mathrm{d} N_{0}}{\mathrm{~d} z}}{\omega^{2}+\lambda^{2}} \sin (\omega t)+\frac{\omega a \frac{\mathrm{d} N_{0}}{\mathrm{~d} z}}{\omega^{2}+\lambda^{2}} \cos (\omega t)
$$

The flux is then given by integrating the covariance over one period

$$
\text { flux }=\int_{0}^{\frac{2 \pi}{\omega}} w^{\prime} N^{\prime} d t=\frac{a \frac{\mathrm{d} N_{0}}{\mathrm{~d} z}}{\omega^{2}+\lambda^{2}} \int_{0}^{\frac{2 \pi}{\omega}} a \sin (\omega t)(-\lambda \sin (\omega t)+\omega \cos (\omega t)) d t=\frac{-a^{2} \frac{\mathrm{d} N_{0}}{\mathrm{~d} z} \lambda \pi}{\omega^{2}+\lambda^{2}}
$$


We can derive the maximum flux explicitly in this equation as $\lambda=\omega$.

\section{A.3.2 Correlation between vertical velocity and nutrient anomaly}

There is an important difference between the flux of a nutrient and the correlation between the nutrient anomaly and the vertical velocity. The correlation between vertical velocity and nutrient anomaly is normalized by the standard deviation of each of those variables

$$
\begin{gathered}
\rho\left(w^{\prime} N^{\prime}\right)=\frac{\left\langle w^{\prime} N^{\prime}\right\rangle}{\sqrt{\left\langle w^{\prime} w^{\prime}\right\rangle} \sqrt{\left\langle N^{\prime} N^{\prime}\right\rangle}} \\
\left\langle N^{\prime} N^{\prime}\right\rangle=\int_{0}^{2 \pi}\left(\frac{-\lambda a \gamma}{\omega^{2}+\lambda^{2}} \sin (\omega t)\right)^{2}+2 \frac{\omega a \gamma}{\omega^{2}+\lambda^{2}} \cos (\omega t) \frac{-\lambda a \gamma}{\omega^{2}+\lambda^{2}} \sin (\omega t)+\left(\frac{\omega a \gamma}{\omega^{2}+\lambda^{2}} \cos (\omega t)\right)^{2} d t \\
\sqrt{\left\langle N^{\prime} N^{\prime}\right\rangle}=\frac{\sqrt{\pi} a \gamma}{\sqrt{\omega^{2}+\lambda^{2}}} \\
\qquad\left(w^{\prime} N^{\prime}\right)=\frac{-a^{2} \gamma \lambda \pi}{\omega^{2}+\lambda^{2}} \frac{\sqrt{\omega^{2}+\lambda^{2}}}{\pi a^{2} \gamma}=-\frac{\lambda}{\sqrt{\omega^{2}+\lambda^{2}}}=a \sqrt{\pi}
\end{gathered}
$$

As expected, the correlation between vertical velocity and nutrient anomaly goes to -1 as the growth rate increases, but the flux is small because $N^{\prime}$ goes to zero.

\section{A.4 Fit between model and theory}

Across all particle trajectories, there is good agreement between the theoretical expectation of the biological rate that maximizes the nutrient flux computed by using a range of biological rates in equation A.21 and the biological rate that maximizes the flux computed by simulating the biological model on the trajectories (Figure A-2).

The model simulation is carried out over a finite time period. One definition of a long 


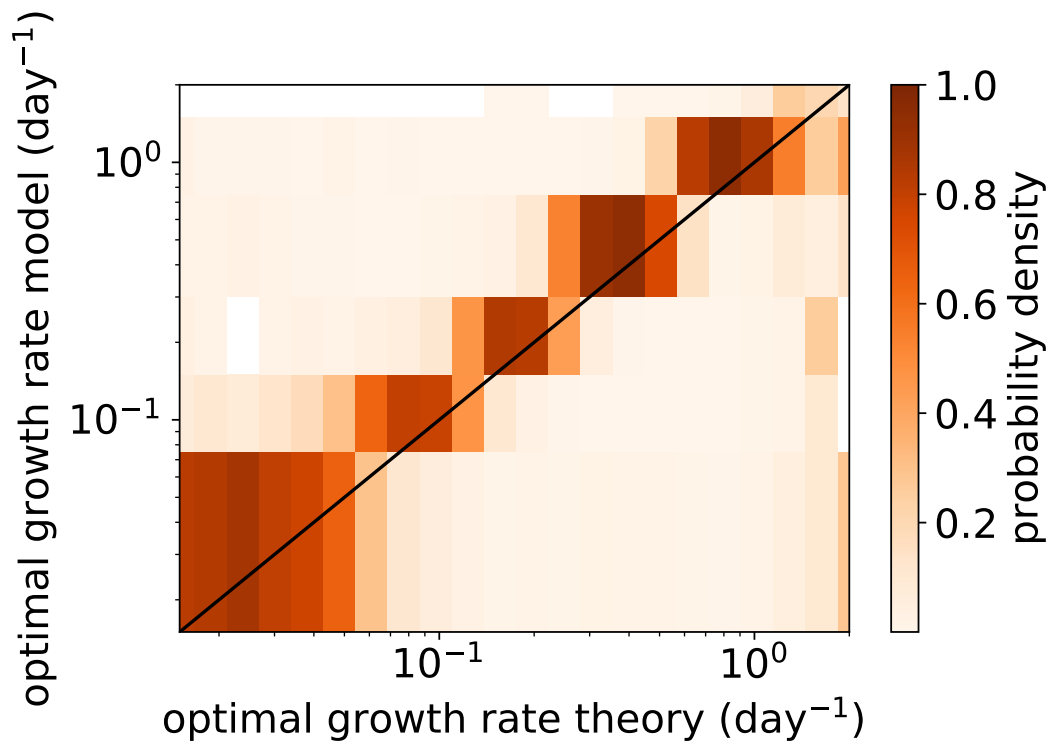

Figure A-2: Histogram of the optimal nutrient flux calculated using equation A.21 ("theory") and on particles in the 3D model. The frequencies are normalized by the number of particles with a given optimum in the theoretical calculation.

enough analysis period is that the vertical velocity is oscillatory in such a way that water parcels complete a full period during the analysis period. We can select Lagrangian trajectories where this is true by selecting trajectories where the net displacement over the analysis period is close to zero (less than $2.5 \mathrm{~m}$ over 30 days). The excluded trajectories have a maximum flux at longer timescales. When we include all trajectories, the flux at slow biological rates increases as does the flux in the absence of biological uptake (Figure A-3B). There is also a long tail on the distribution of rates that maximize the flux (Figure A-3A).

One of the major assumptions about the outcome of the model is that there is relatively little variation in the vertical velocity and background nutrient concentration gradient over the mean free path of trajectory allowing us to assume that the nutrient anomaly is linear with depth. Solutions could be obtained for other nutrient anomaly profiles as well but are not derived here. Examining histograms of the nutrient anomaly as a function of depth, we see that this assumption is generally consistent with the model (Figure A-4). With the slower growth rates, the nutrient anomaly has the same depth dependence as the background profile $N_{0}(z)$. There are a few extreme cases where this assumption is not true with the faster growth rates. 

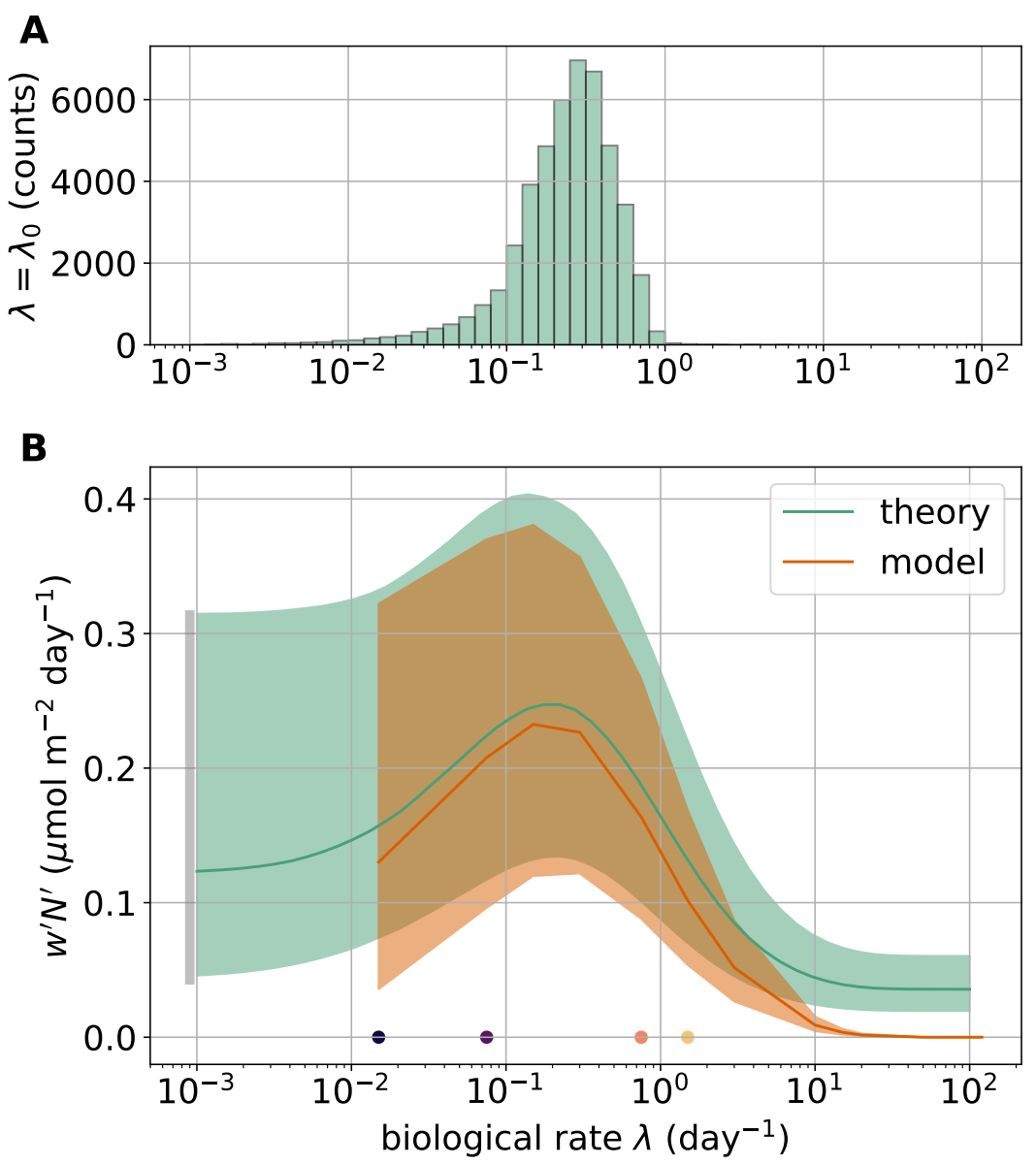

Figure A-3: (A) Distribution of rates $\lambda$ that maximizes the nutrient flux. (B) Nutrient flux as a function of biological rate on Lagrangian trajectories (orange curve) and theoretical expectation of the nutrient flux from equation 3 (green curve). The solid line is the median of all trajectories shading shows the interquartile range. The grey bar on the ordinate shows the interquartile range of the vertical eddy diffusion in the absence of biological uptake.
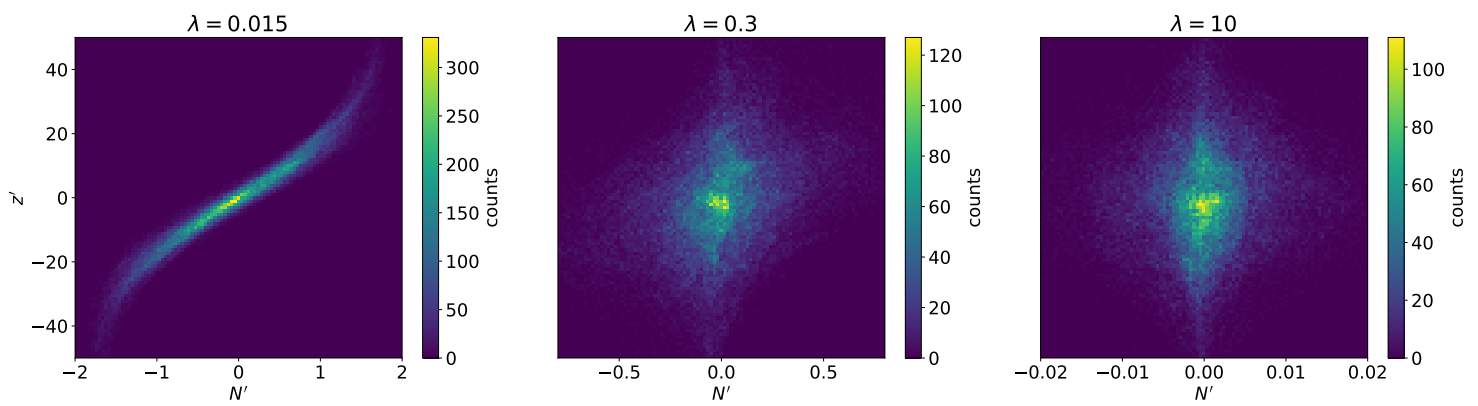

Figure A-4: Nutrient anomaly as a function of the depth anomaly (vertical distance from z $=-155 \mathrm{~m}$ ) for a range of biological rates. 
If we reduce the slope of the background nutrient profile, the variations in the magnitude of the vertical velocity with depth become more important and the nutrient anomaly is less linear with depth. For example, we reduce the magnitude of the background nutrient

anomaly by changing the value of $A$ in the function $N_{0}(z)=A\left(1-\tanh \left(\frac{z-N_{\text {cline }}}{30}\right)\right)$. In most model simulations, we use $A=2.5$ for a deep nutrient concentration of 5 . To reduce the slope of the nutricline, we use $A=1$ which only gives a deep nutrient concentration of 2. With a smaller $A$, the average flux across all water parcels displays a similar shape as in Figure A-3, but the fluxes are smaller magnitude, the flux at high biological rates is negative, and, notably, the variation among trajectories increases (Figure A-5).

\section{A.5 General analysis of the maximum flux}

For any given vertical velocity timeseries equation A.21 can be used to derive the dependence of the flux on the biological rate. For a general vertical velocity time series, the maximum of the flux curve as a function of the biological rate is given by

$$
\frac{\partial w^{\prime} N^{\prime}}{\partial \lambda}=-\frac{\mathrm{d} N_{0}}{\mathrm{~d} z} \int_{t}^{\infty}-\tau e^{-\lambda \tau} w(t-\tau) w(t) d \tau+C_{1}=0
$$

where $\tau=t-t^{\prime}$. The autocorrelation function relates the vertical velocity and flux characteristics. The effects of longer timescales are amplified in determining the biological rate that maximizes the flux due to $\tau$ term in the derivative. While there are many possible autocorrelation functions that result in a given biological rate that maximizes the flux, in general the first zero crossing of the autocorrelation is at a shorter time lag with a faster biological rate (Figures A-6 and A-7). The autocorrelation function is in the time domain and is related to the vertical velocity spectrum in the frequency domain according to the Wiener-Khinchine theorem. 

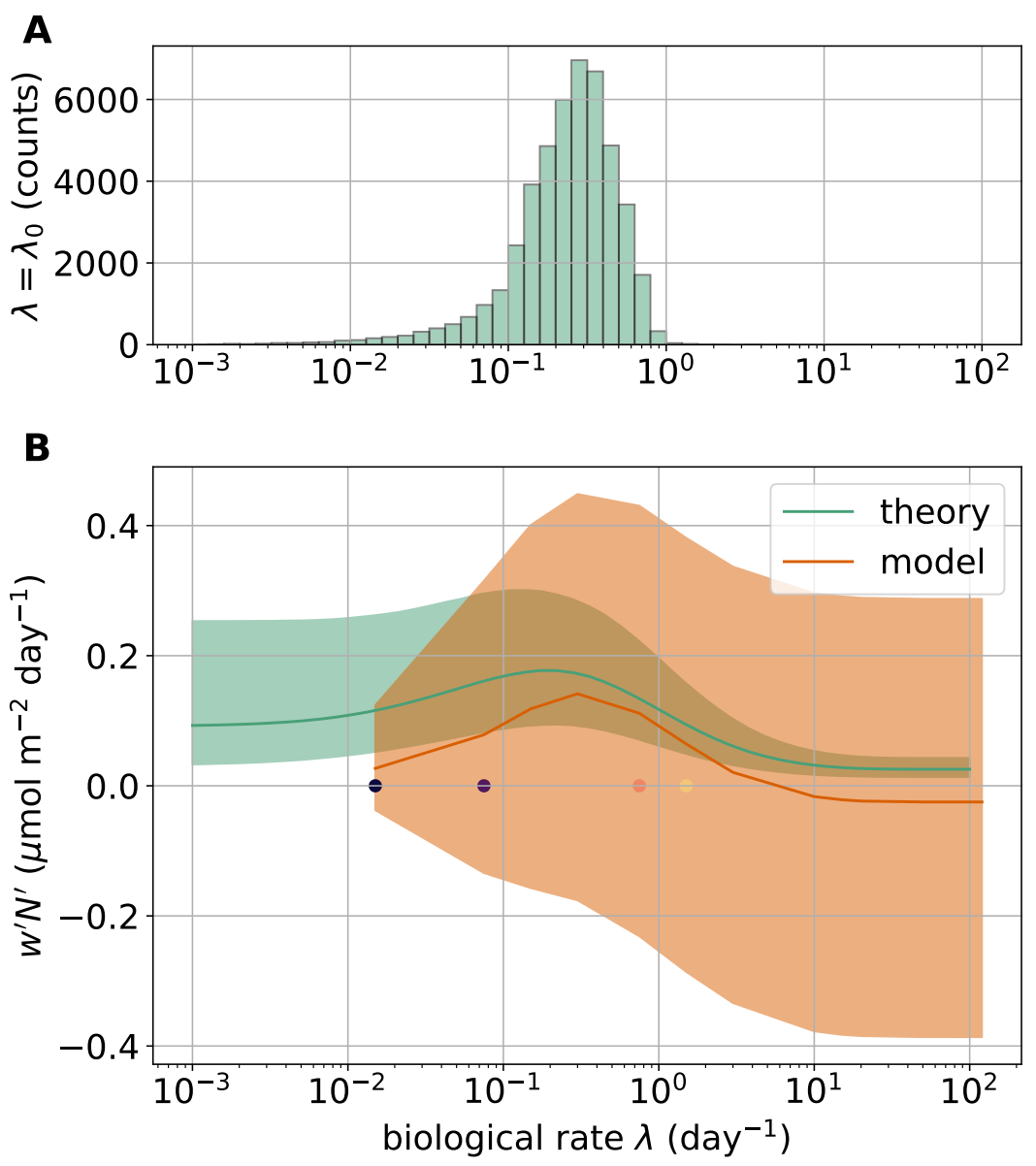

Figure A-5: (A) Distribution of rates $\lambda$ that maximizes the nutrient flux. (B) Nutrient flux as a function of biological rate on Lagrangian trajectories (orange curve) and theoretical expectation of the nutrient flux from equation 3 (green curve). The solid line is the median of all trajectories shading shows the interquartile range. The grey bar on the ordinate shows the interquartile range of the vertical eddy diffusion in the absence of biological uptake. 


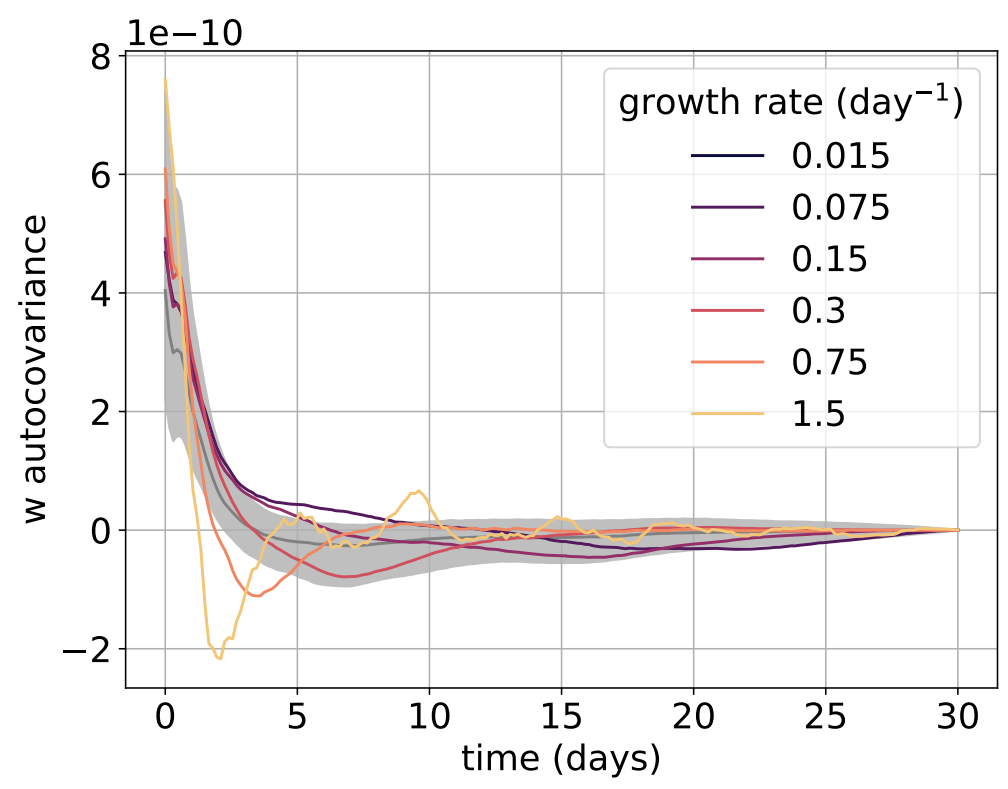

Figure A-6: Vertical velocity autocorrelation as a function of time. The grey curve shows the median and interquartile range of all trajectories. The colored lines show the median autocorrelation across all trajectories with a given biological rate the maximizes the flux.

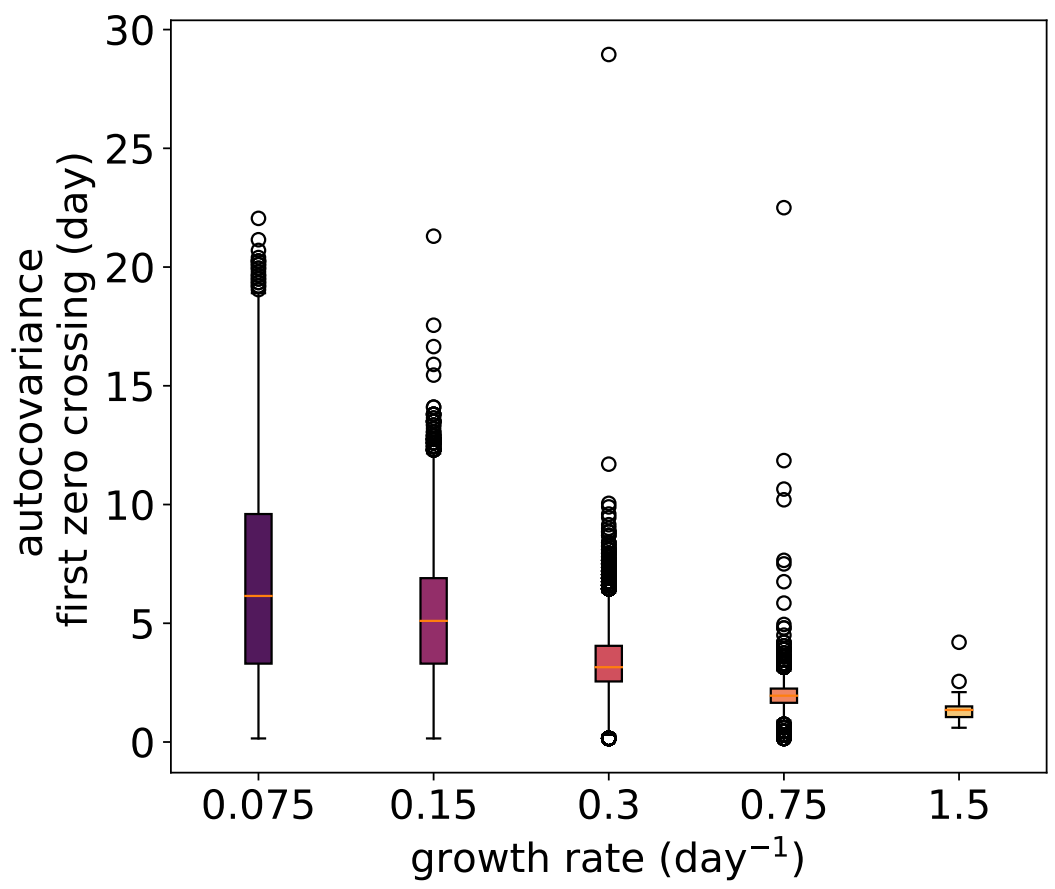

Figure A-7: First zero crossing of the vertical velocity autocorrelation. The biological rate on the abscissa is the rate the maximizes the vertical flux. The box plots show the distributions of first zero crossings across all trajectories. 


\section{Appendix B}

\section{Patchiness as a function of biological}

\section{rate}

The Lagrangian analysis in chapter 4 is entirely within the time domain. However, Lagrangian observations are rare while spatial observations, especially from satellite imagery of the sea surface are common. This imagery of the sea surface provided some of the early evidence that submesoscale dynamics affect biological production. In addition, there are well-established theories for the wavenumber spectra of tracers as they relate to different dynamics but such theories for frequency spectra are limited to internal waves. Therefore, translating the results back into the spatial domain is useful for comparison with the literature.

The relationship between the spatial and temporal characteristics of the vertical kinetic energy (VKE) can be examined in the wavenumber-frequency space (Figure B-1A); the VKE lies on the non-dispersive line $\omega=c k$ with phase speed $17 \mathrm{~km} /$ day. This relatively fast phase speed is likely due to the influence of strong mesoscale eddies on the vertical velocity distributions. For large $\lambda$ (fast nutrient uptake), the spatial and temporal scale of nutrient uptake $\left(\lambda N^{\prime}\right)$ is similar to the vertical velocity (Figure B-1B). However, for small $\lambda$ (slow uptake) the nutrient uptake occupies a different region of the wavenumber-frequency space than the vertical kinetic energy.

The origins of the biological patchiness observed in satellite images is debated in the 
literature. The results presented in this thesis resolve the debates by highlighting the existence of two types of patchiness that correspond to the extremes of the distribution of flux as a function of biological rate. Nutrient anomalies are injected at the scale of the vertical velocity variability, which for large $\lambda$ results in small-scale patchiness in the nutrient flux and new production. This is the type of patchiness discussed in Mahadevan and Campbell (2002). By contrast, for small $\lambda$ (slow uptake), the nutrient fluxes are preferentially at lower frequencies and larger spatial scales and the tracer anomalies are stirred by the horizontal velocity field to smaller scales resulting in a filamentous distribution of nutrient and biomass Abraham (1998). However, the filaments in nutrient anomaly do not propagate in the same way as the vertical velocity, so the vertical velocity and tracer anomaly are no longer in phase and the tracer flux is weak (Figure B-1). 

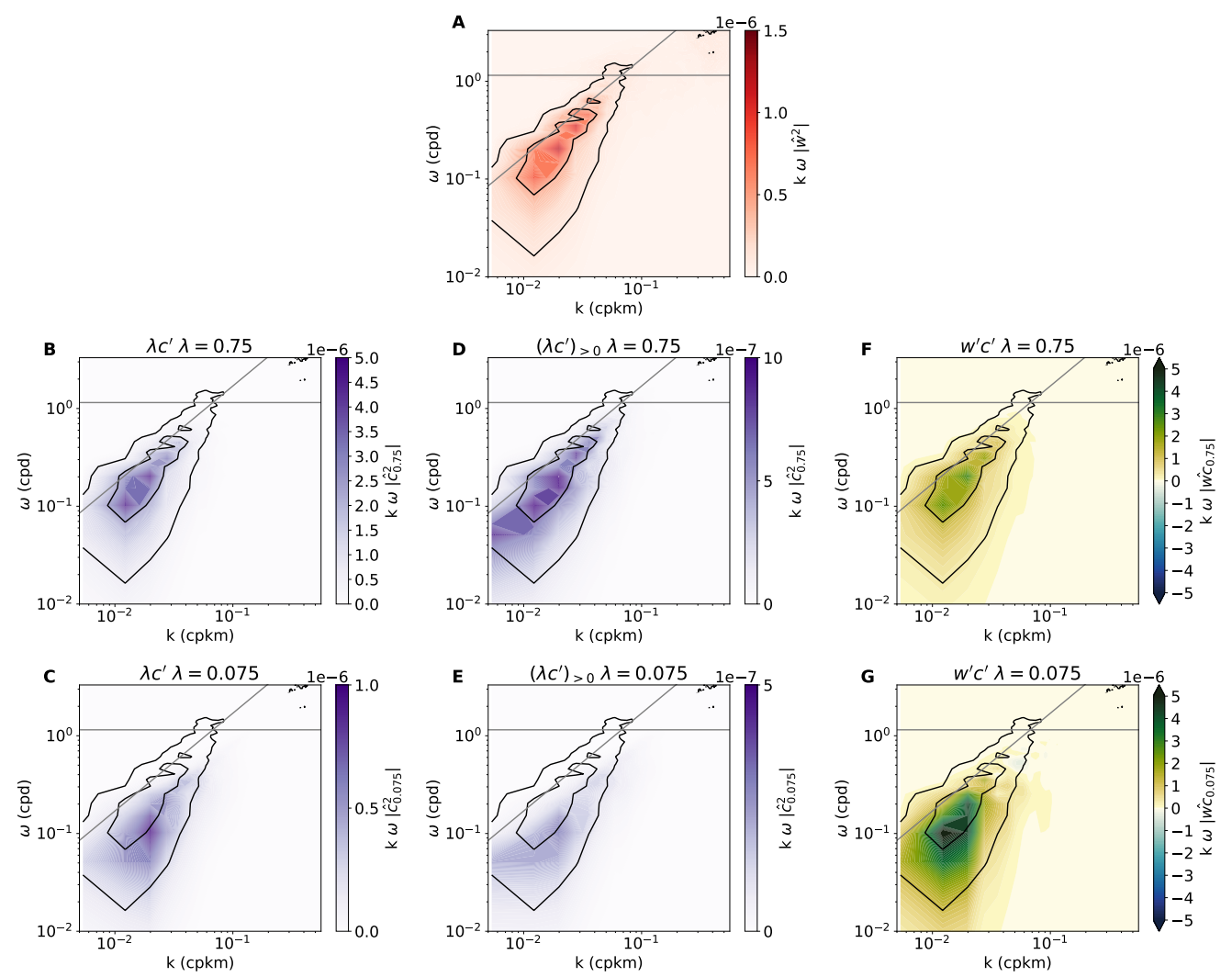

Figure B-1: (A) Wavenumber-frequency spectrum of the vertical kinetic energy at 117 meters. (B, C) Wavenumber-frequency of the biological reaction term $\left(\lambda c^{\prime}\right)$ at 117 meters with (B) a relatively fast growth rate $(\lambda=0.75)$ and $(\mathrm{C})$ a relatively slow growth rate $(\lambda=0.075)$. $(\mathrm{D}, \mathrm{E})$ Wavenumber-frequency of the new production, the positive part of $\lambda c^{\prime}$, at 117 meters with (D) a relatively fast growth rate $(\lambda=0.75)$ and $(\mathrm{E})$ a relatively slow growth rate $(\lambda=0.075)$. (F, G) Wavenumber-frequency of the nutrient flux $\left(\lambda c^{\prime}\right)$ at 117 meters with $(\mathrm{F})$ a relatively fast growth rate $(\lambda=0.75)$ and $(\mathrm{G})$ a relatively slow growth rate $(\lambda=0.075)$. On each panel the grey horizontal line is at the inertial frequency. The grey diagonal line is a non-dispersive line with a phase speed of $17 \mathrm{~km} /$ day. The black contours are level curves of the vertical velocity wavenumber-frequency spectrum. 


\section{Appendix C}

\section{Observations of flux dependence on biological timescale}

\section{C.1 Model set-up}

The background nutrient profile is based on observations. Due to the linear nature of the model and the modeling choice that $\lambda$ is constant with depth, all production will occur where there is a gradient in the background nutrient profile. We ensure that the production is localized in the region of the deep chlorophyll maximum at BATS by setting up the function $N_{0}(z)$ such that its derivative has the same shape as the chlorophyll profile at BATS (figure C-1).

The resulting nutrient profile $N_{0}(z)$ is similar to the profile in the subtropical gyres, although it is notable that the nutricline at the Hawaii Ocean Timeseries (HOT) Station Aloha is steeper than the nutricline at BATS (Figure C-2).

\section{C.2 North Pacific comparison}

The North Pacific subtropical gyre is stratified and nutrient limited throughout the year. In this region, productivity has been observed to be influenced by mesoscale eddies Letelier et al. (2000); Sakamoto et al. (2004); White et al. (2007). In the observational comparison in figure 6, we average over all data because the monthly station resolution is not sufficient 
to capture submesoscale frequencies. By averaging over all available data, we are able to include 15 data points per biological rate. However, by doing the averages this way we risk the inclusion of correlations with growth rate that overwhelm the signal of the process that we have proposed. One of the major potential issues could be the seasonal cycle. There is some seasonality to the system with the highest temperatures and the highest growth rates observed during the summertime Church et al. (2006), although the seasonality in carbonspecific growth rate at 120 meters is weak (Figure C-3). The mesoscale eddy kinetic energy measured from satellites has a maximum in April-May Chen and Qiu (2010). The higher summertime eddy activity could increase the observed nutrient anomaly during times when the growth rate is also high, but we find instead that there are relatively small nutrient anomalies during the summer. The diffusive transport of carbon is constant throughout the year although the lateral transport may vary throughout the year Keeling et al. (2004). However, the mixed layers are deeper in the wintertime, potentially allowing submesoscale features to reach into the nutricline during the winter. We do not present any error bars because the theory presented is for the average flux. We show all data in figure C-4. There are 2 extreme value of nutrient flux that are due to large nutrient anomalies. These are removed before computing the average curve. 


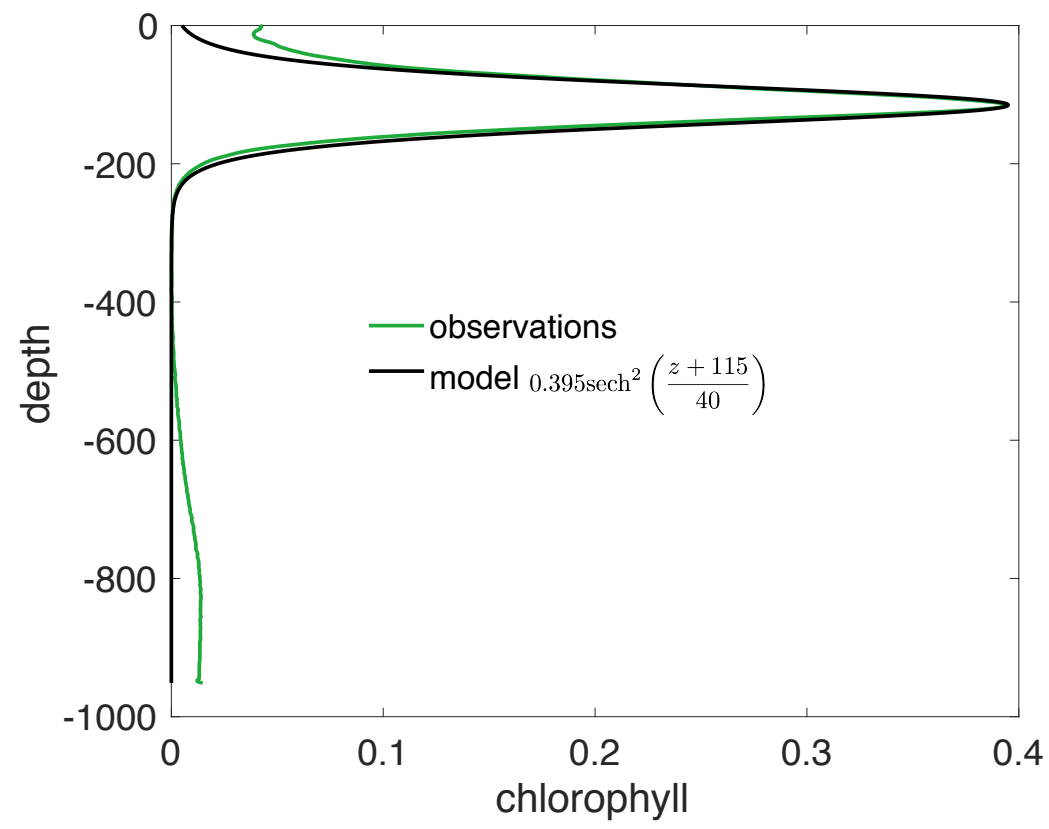

Figure C-1: Chlorophyll as a function of depth observed at BATS in June (green) and $\frac{\mathrm{d} N_{0}}{\mathrm{~d} z}$

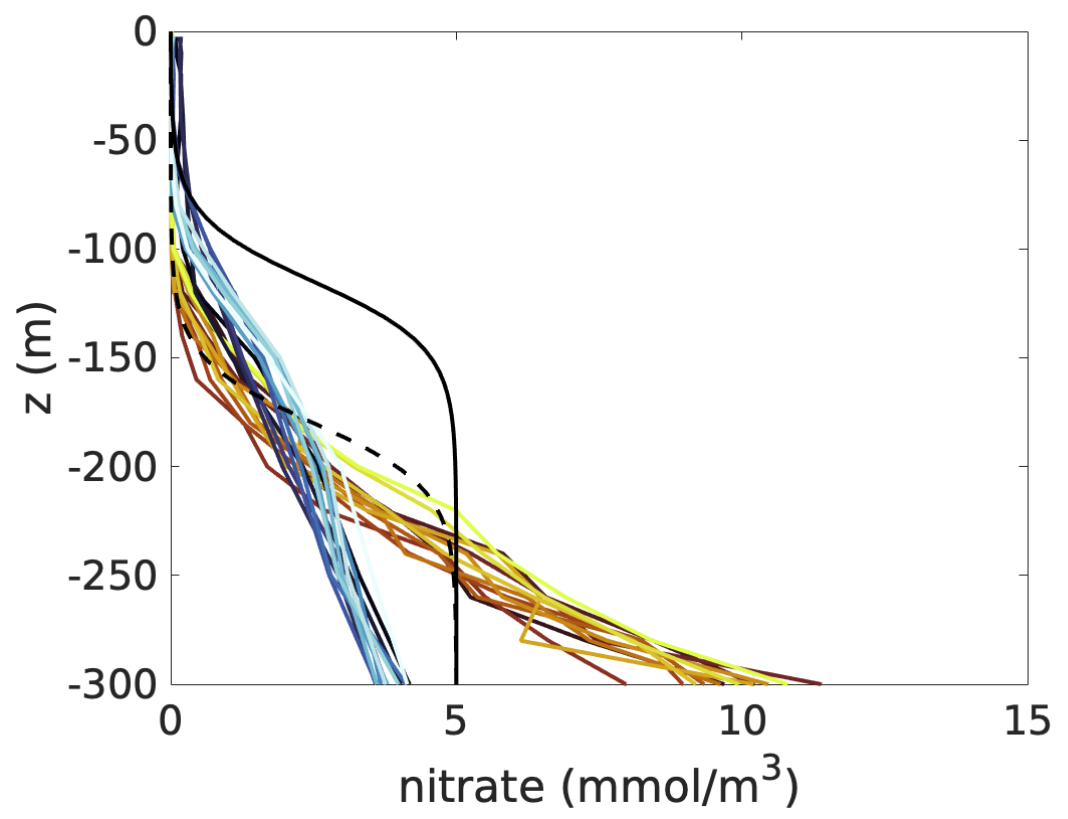

Figure C-2: Model and observational nutrient profiles. The model equilibrium profile is the black line and the dashed line is a shifted profile to match the nutricline depth at HOT. The observational profiles are monthly mean profiles from the Bermuda Atlantic Time Series (blues) and Hawaii Ocean Timeseries (oranges). The colors vary from dark to light with month from January to December. The model profile has no gradient below the maximum euphotic depth so that there is no productivity below this depth, due to the model simplifications. The model has a larger nutrient gradient in the nutricline than do the observations at BATS. 

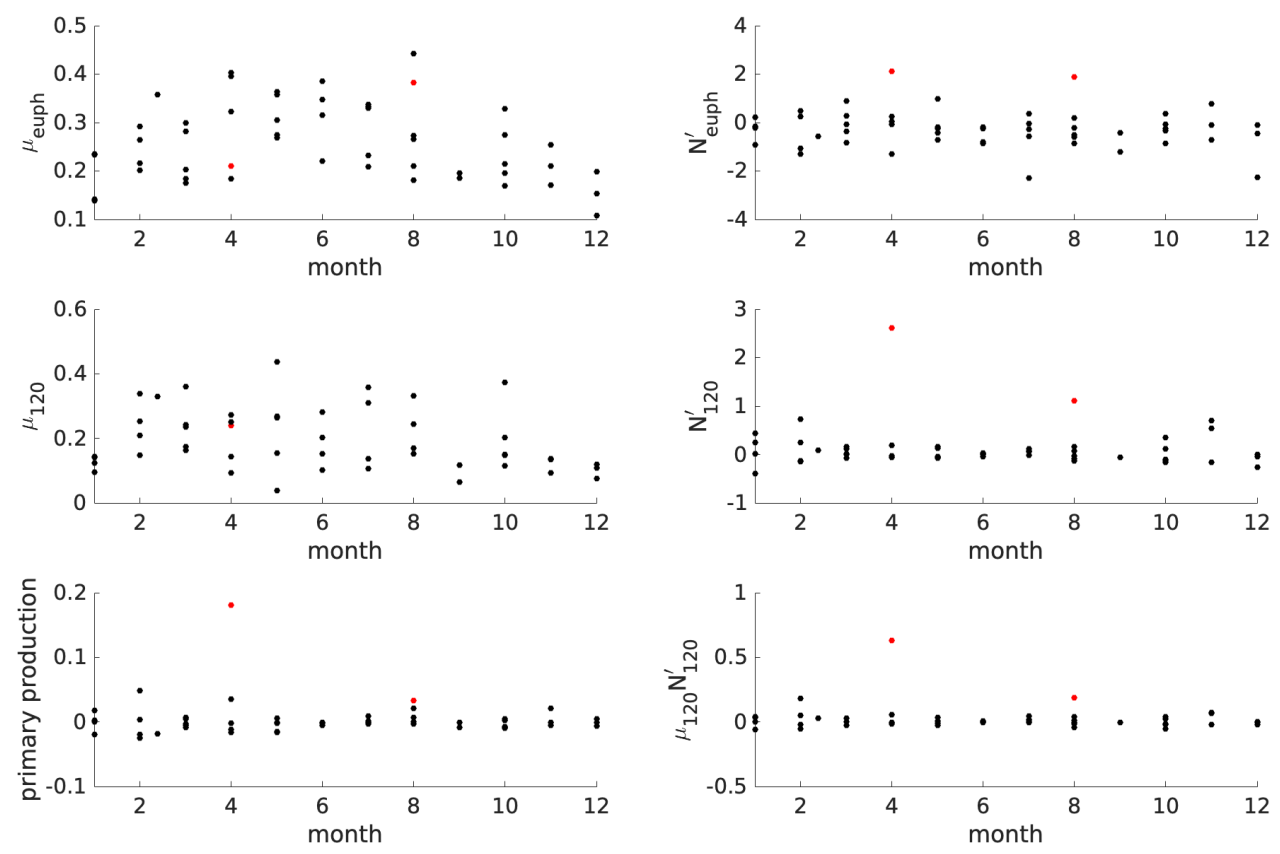

Figure C-3: From top to bottom, left to right, measured and computed quantities as a function of month, euphotic zone average carbon specific growth rate, carbon specific growth rate at 120 meters, euphotic zone average primary production, average nutrient anomaly in the euphotic zone, average nutrient anomaly at $120 \mathrm{~m}$, nutrient flux calculated as $\mu N^{\prime}$ at $120 \mathrm{~m}$. The black dots show the most extreme values of nutrient anomaly. 

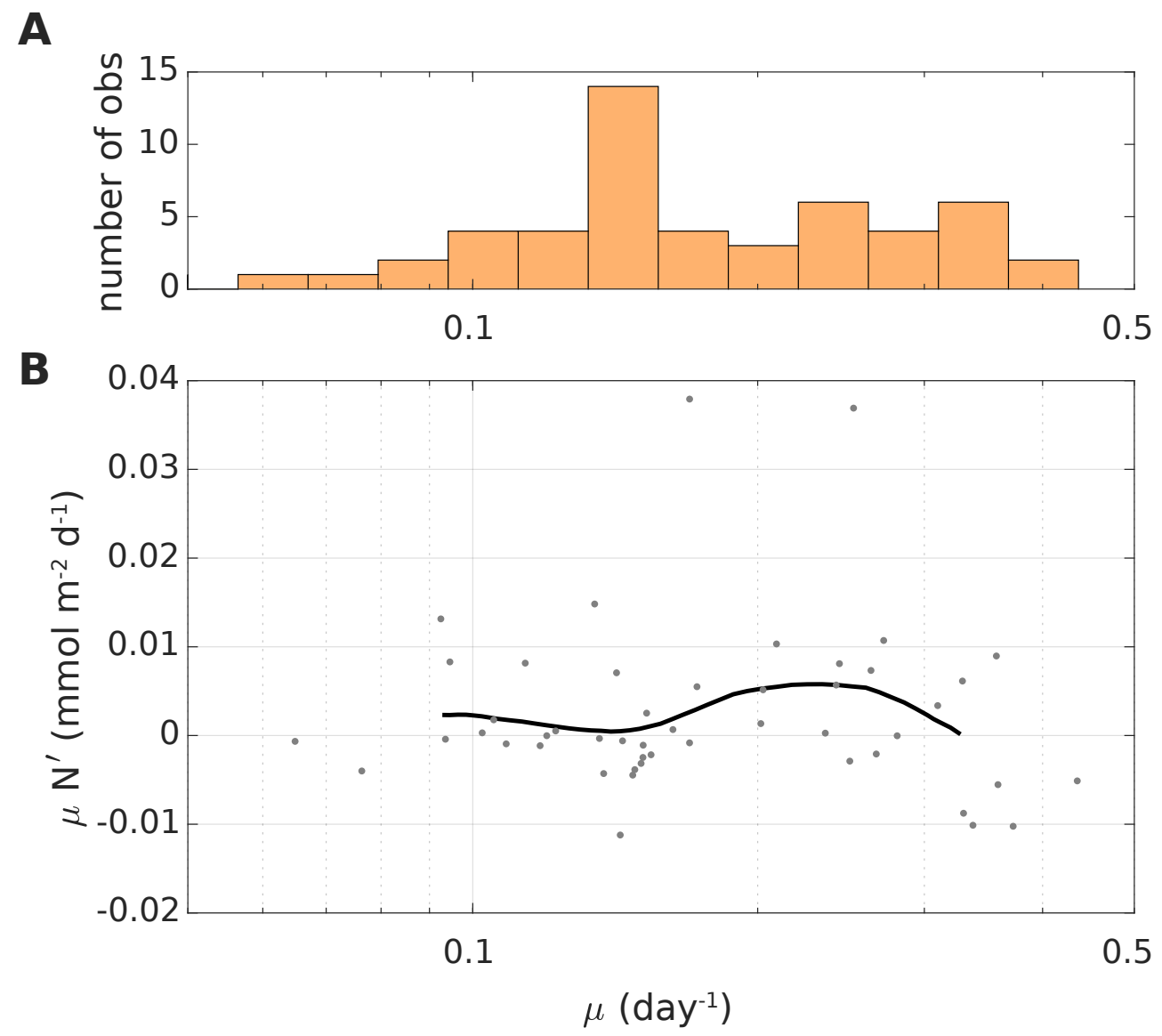

Figure C-4: Scatter plot of all observations of the estimated nutrient flux $\left(\mu N^{\prime}\right)$ as a function of carbon-specific growth rate $(\mu)$. 
Appendix D

\section{Tracer analysis of mixed layer subduction}




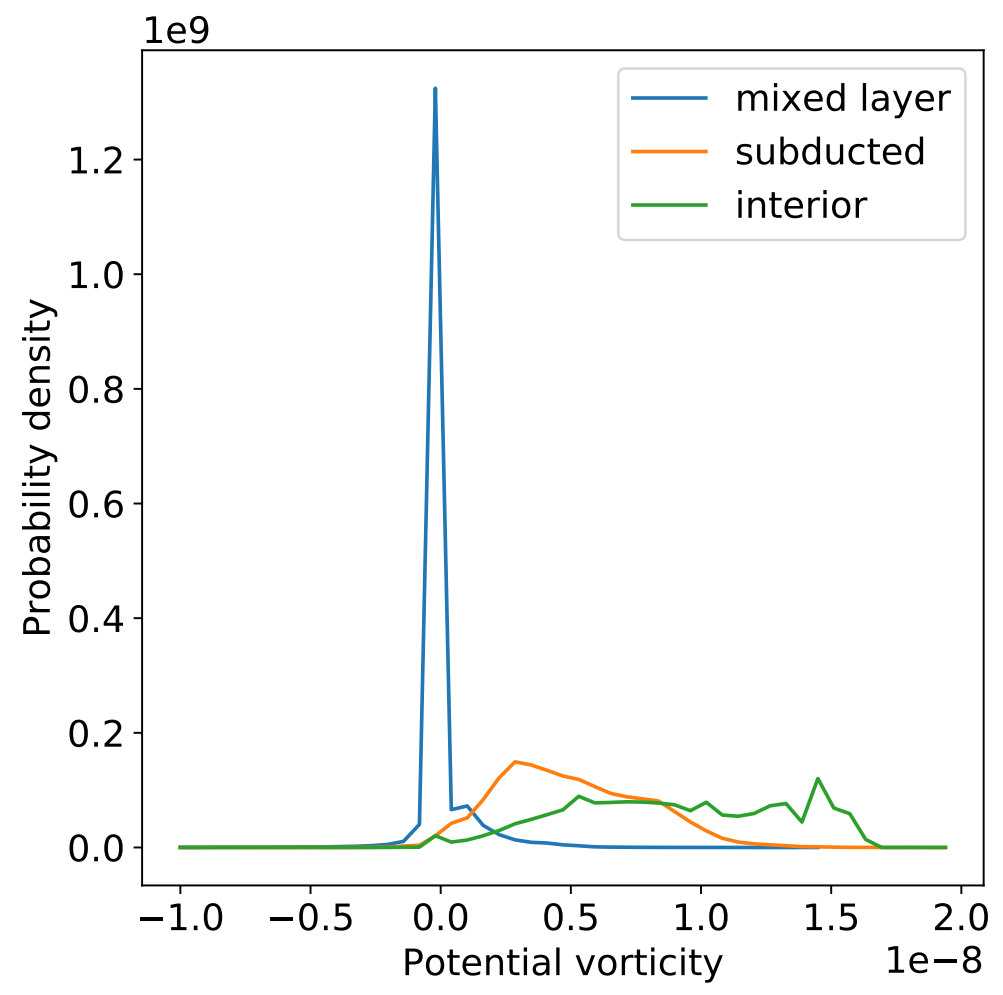

Figure D-1: PV probability density functions on model day 50 in the mixed layer (blue line), on outcropping isopycnals, but below the mixed layer (green line), and of water that subducted from the mixed layer during an offline tracer experiment that started on model day 43.75 (orange line).

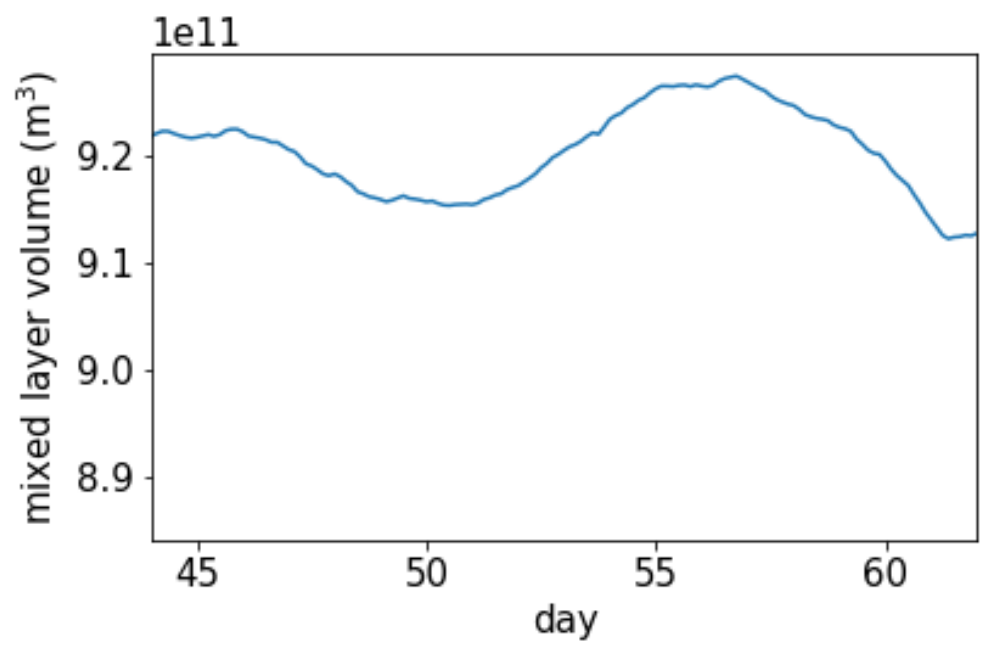

Figure D-2: Volume of the mixed layer through the analysis period. The mixed layer volume is fairly constant throughout the analysis period, indicating that there is little restratification. 


\section{Appendix E}

\section{Estimation of POC from flow}

\section{cytometry and bio-optical properties}
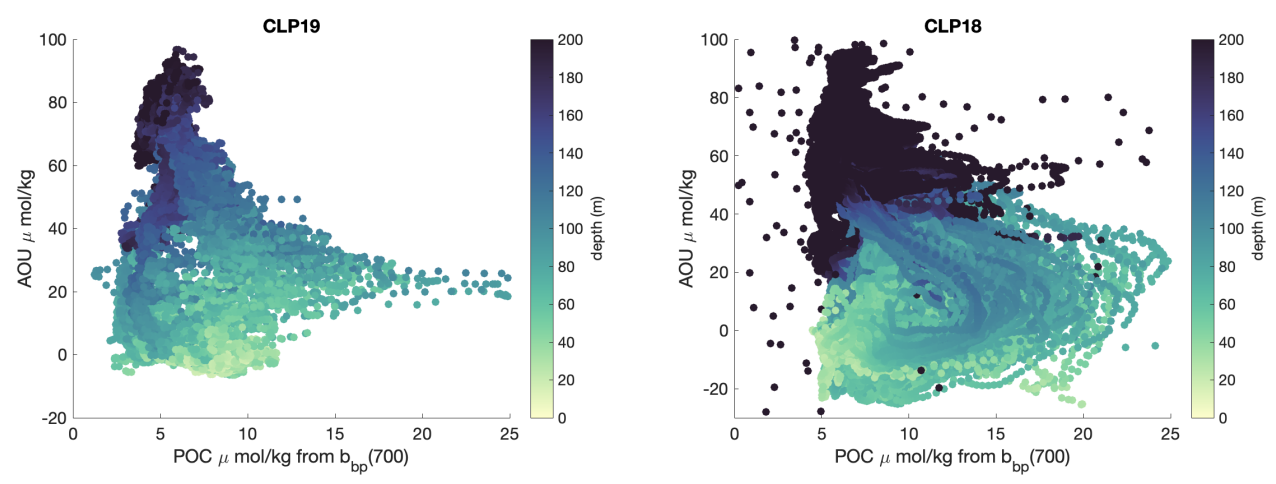

Figure E-1: POC estimated from $b_{b p}(700)$ on the ecoCTD using the conversion relationship from Mayot et al. (2017) plotted against AOU for CLP18 and CLP19. Each point is a sample and is colored with the sample depth. 


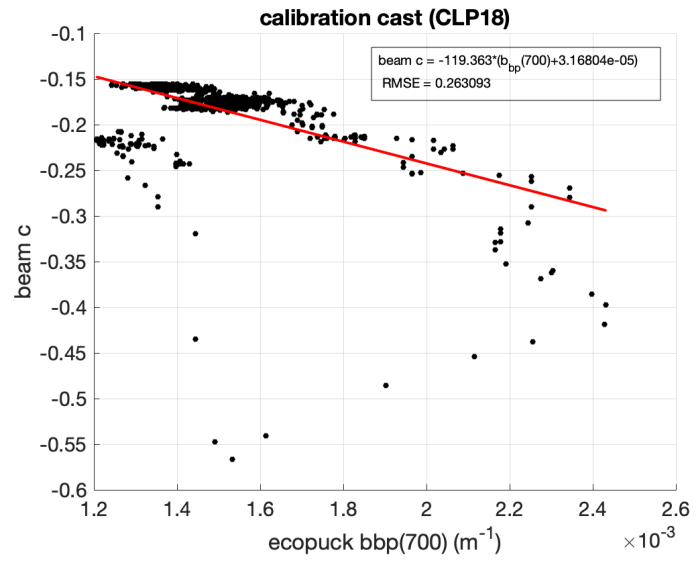

Figure E-2: Correlation between ship-board CTD $c_{p}$ and ecoCTD $b_{b p}(700)$ from the calibration cast on the CLP18 cruise. The beam c values should not be negative. I think they need to be calibrated against the dark values. 
Appendix F

Supplemental biological data analysis

figures 


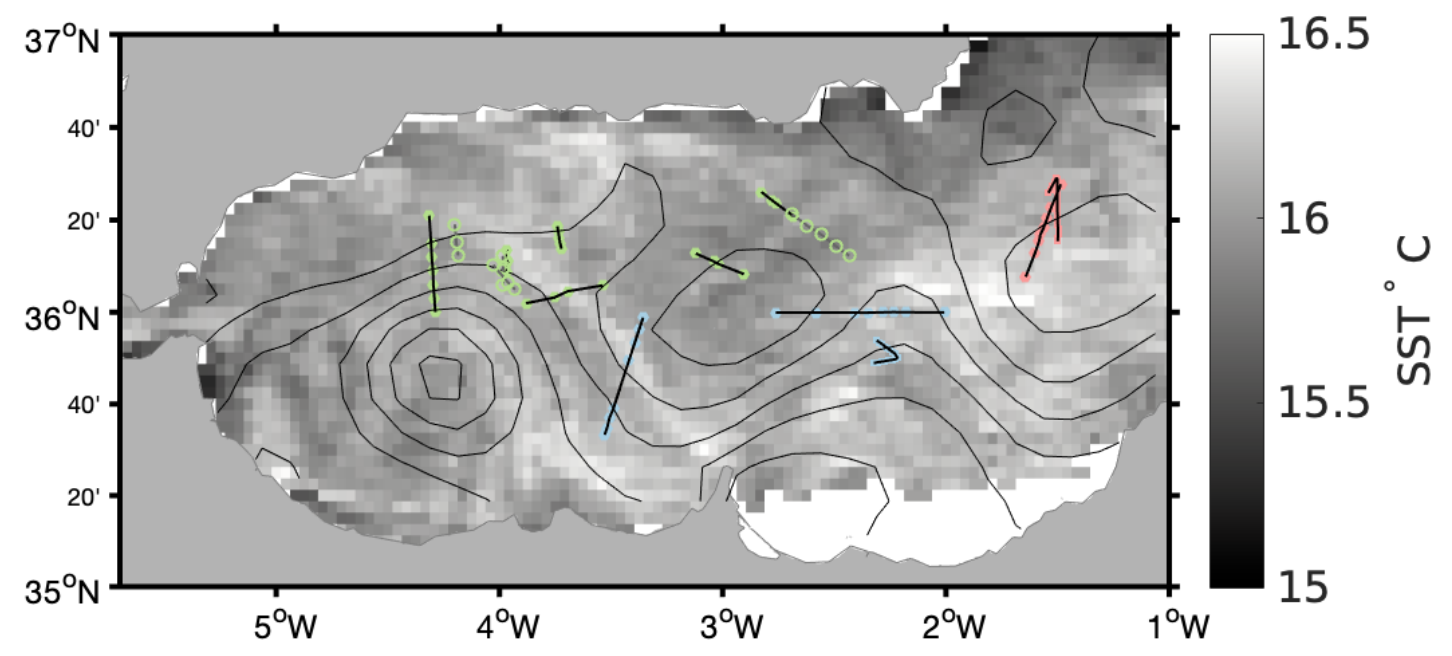

Figure F-1: Spatial distribution of transects as in Figure 5-1 but with the background of satellite sea surface temperature. The transect from IRENE is pink, the transects from CLP18 are blue, and the transects from CLP19 are green. 


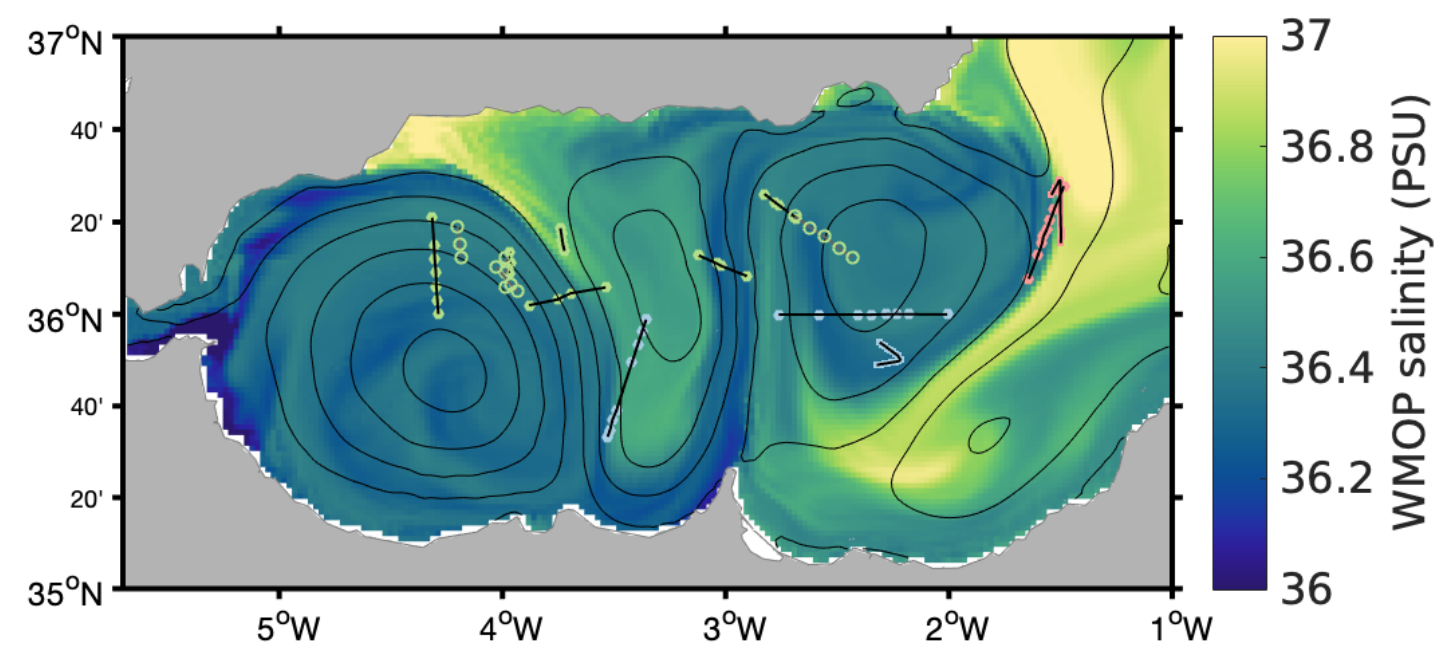

Figure F-2: Spatial distribution of transects as in Figure 5-1 but with the background of WMOP model salinity and sea surface height from May 31, 2018. The transect from IRENE is pink, the transects from CLP18 are blue, and the transects from CLP19 are green.

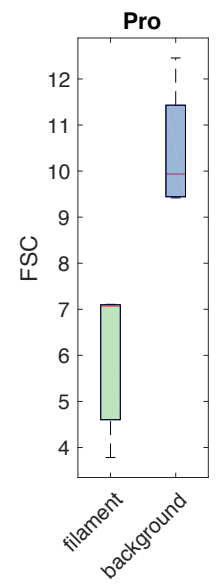

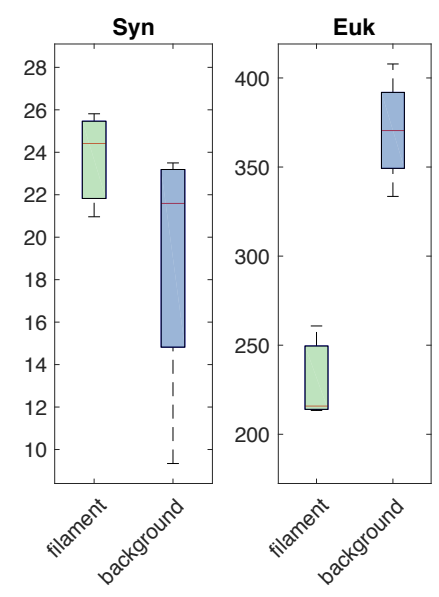

(a) Anticyclonic eddy
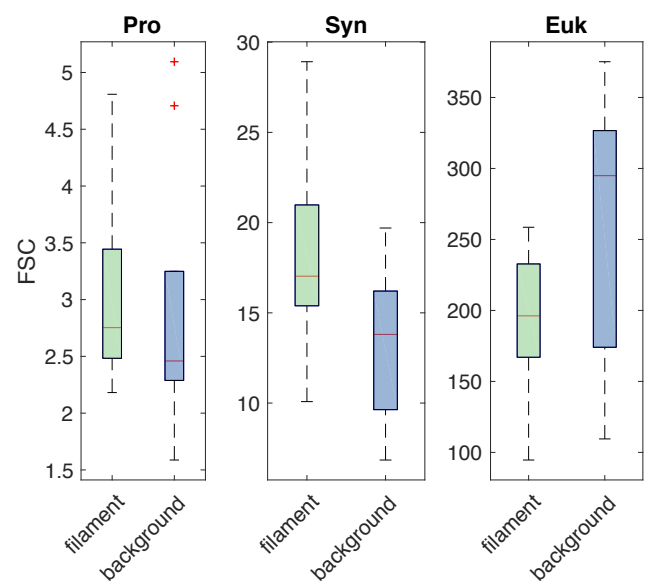

(b) Western Alboran Gyre

Figure F-3: Distribution of forward scattering of each taxonomic group from flow cytometry in the filaments and background below $100 \mathrm{~m}$ in each region during CLP19. 

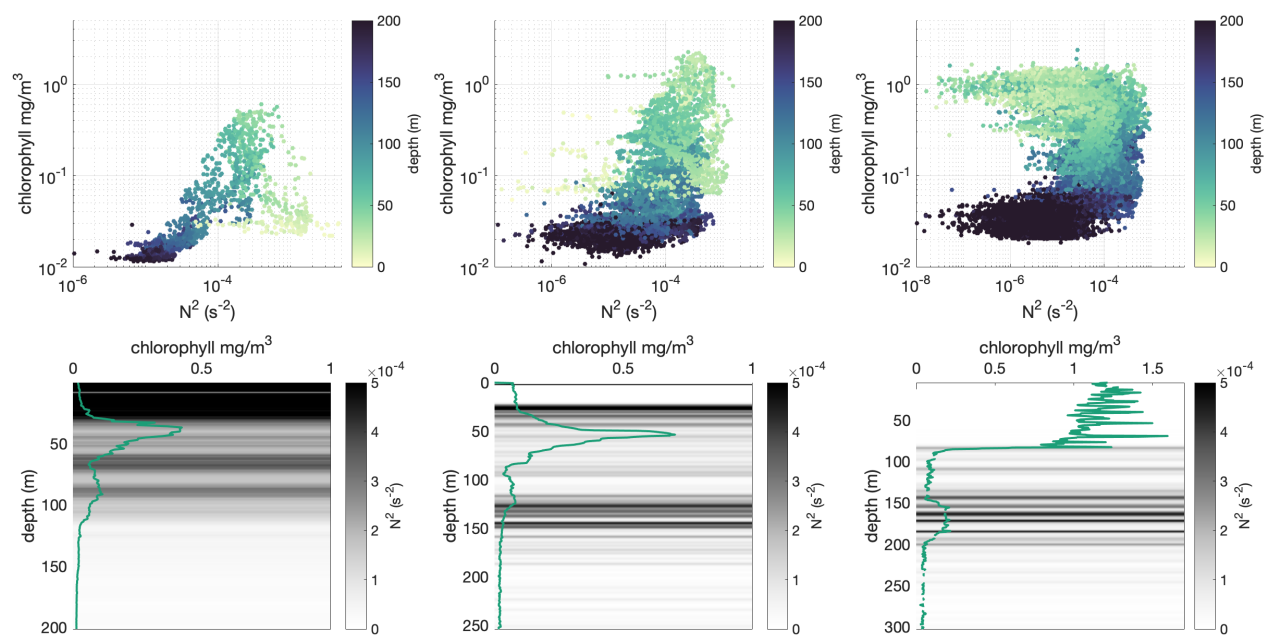

Figure F-4: From left to right IRENE, CLP18, CLP19. The upper panels show chlorophyll concentration plotted against stratification. The lower panels show example chlorophyll profiles. The background of the panels shows the stratification on that profile. Each of these profiles has a secondary chlorophyll maximum.
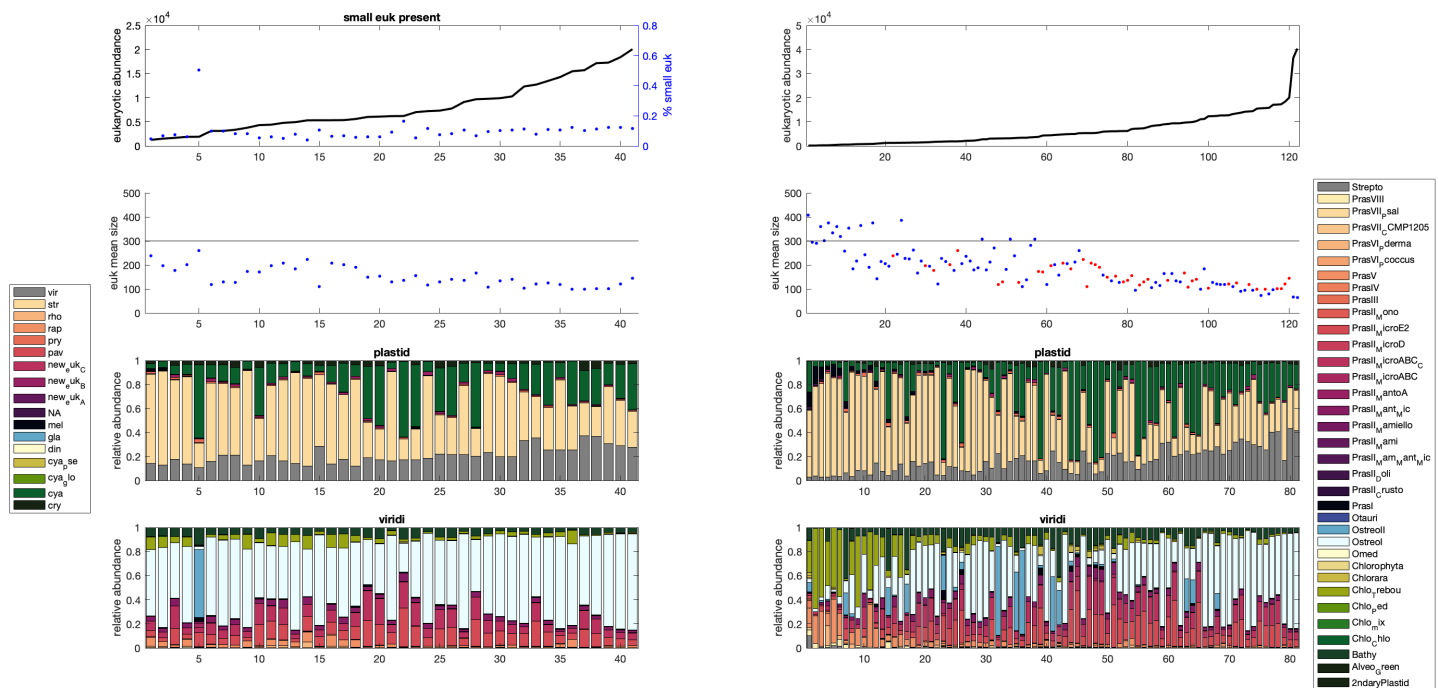

Figure F-5: Combined analysis of flow cytometry and amplicon sequencing. The top row is the eukaryotic abundance from flow cytometry. The second row is the eukaryotic cell size from flow cytometry. The third row shows the relative abundance of plastids (left legend) with the samples arranged in the same order as the upper panels. The bottom row shows the relative abundance of Prasinophytes from amplicon sequencing. The left column shows only samples where I identified a population of small picoeukaryotes in the flow cytometry cytograms. The right column shows all samples from CLP19 with the samples in the left column colored in red in the second row. 

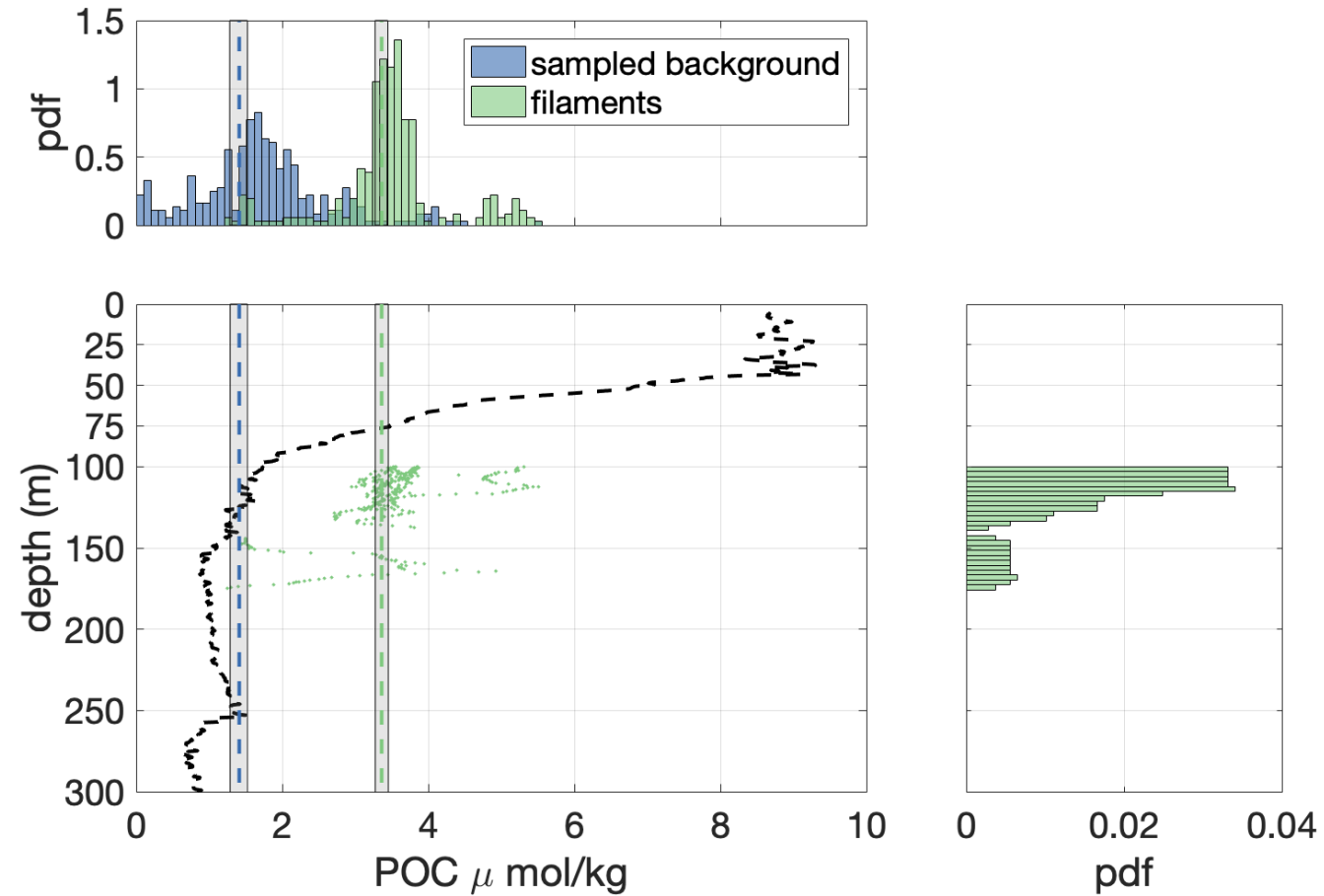

Figure F-6: POC export during CLP18. (top) Probability density functions of POC concentration within the intrusions (green) and POC concentration randomly sampled with the same depth distribution as the intrusions but outside the intrusions. The dashed line shows the geometric mean of the POC distribution in the gyre intrusion and background. The dotted line shows the mean of the POC distribution in the eddy intrusion and background. The grey shading shows the $95 \%$ confidence interval. These averages are also shown in the panel below. (bottom left) Average POC profile in the gyre (dashed) and eddy (dotted). The scatter points show the intrusion POC concentrations at each depth below $100 \mathrm{~m}$. The darker points are in the eddy and the lighter points are in the gyre. (right) Depth distribution of intrusion samples below $100 \mathrm{~m}$. 

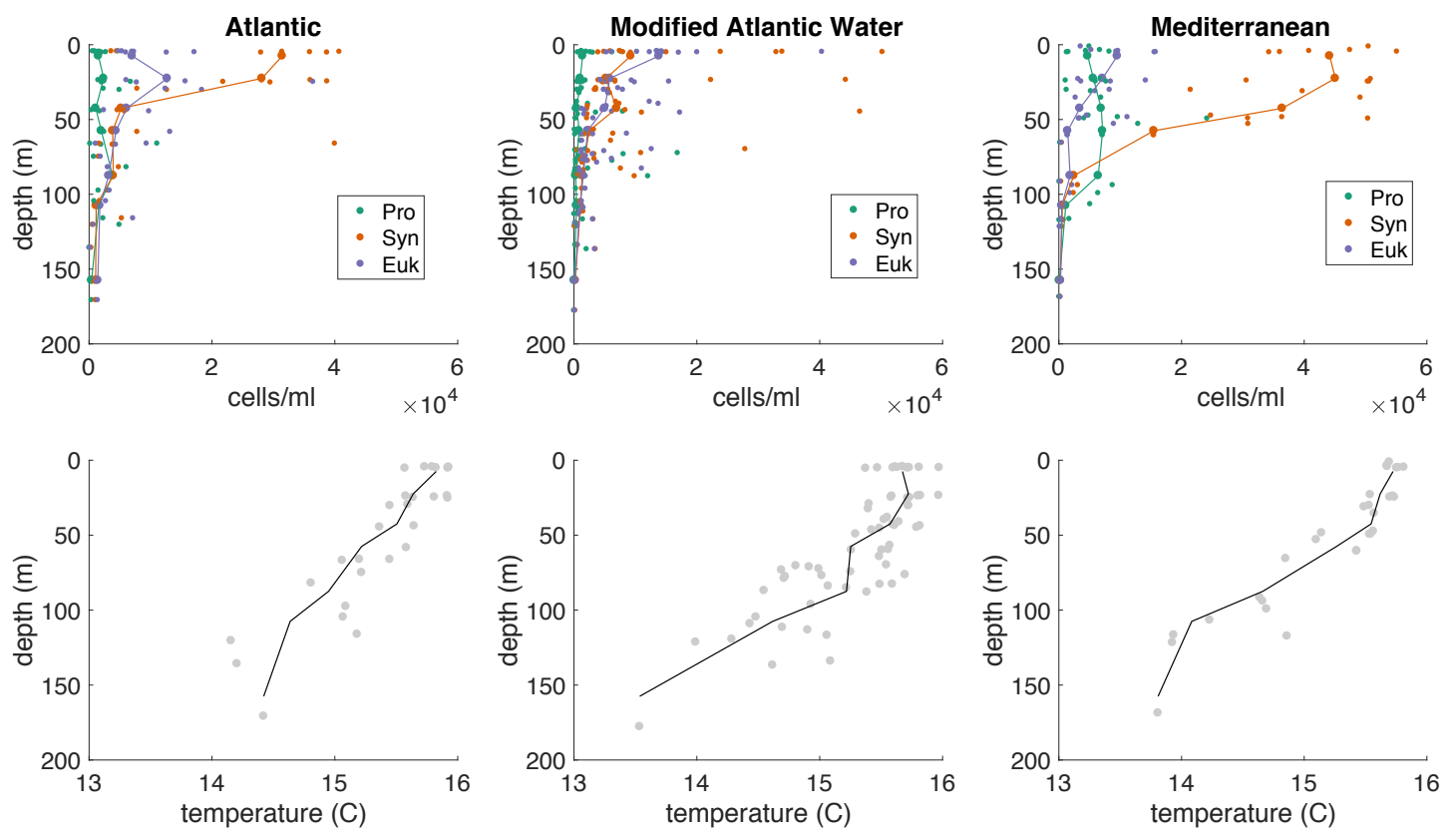

Figure F-7: (upper) Average profiles of photosynthetic cell counts in each water mass from CLP19. (lower) Average temperature profiles for each water mass. 


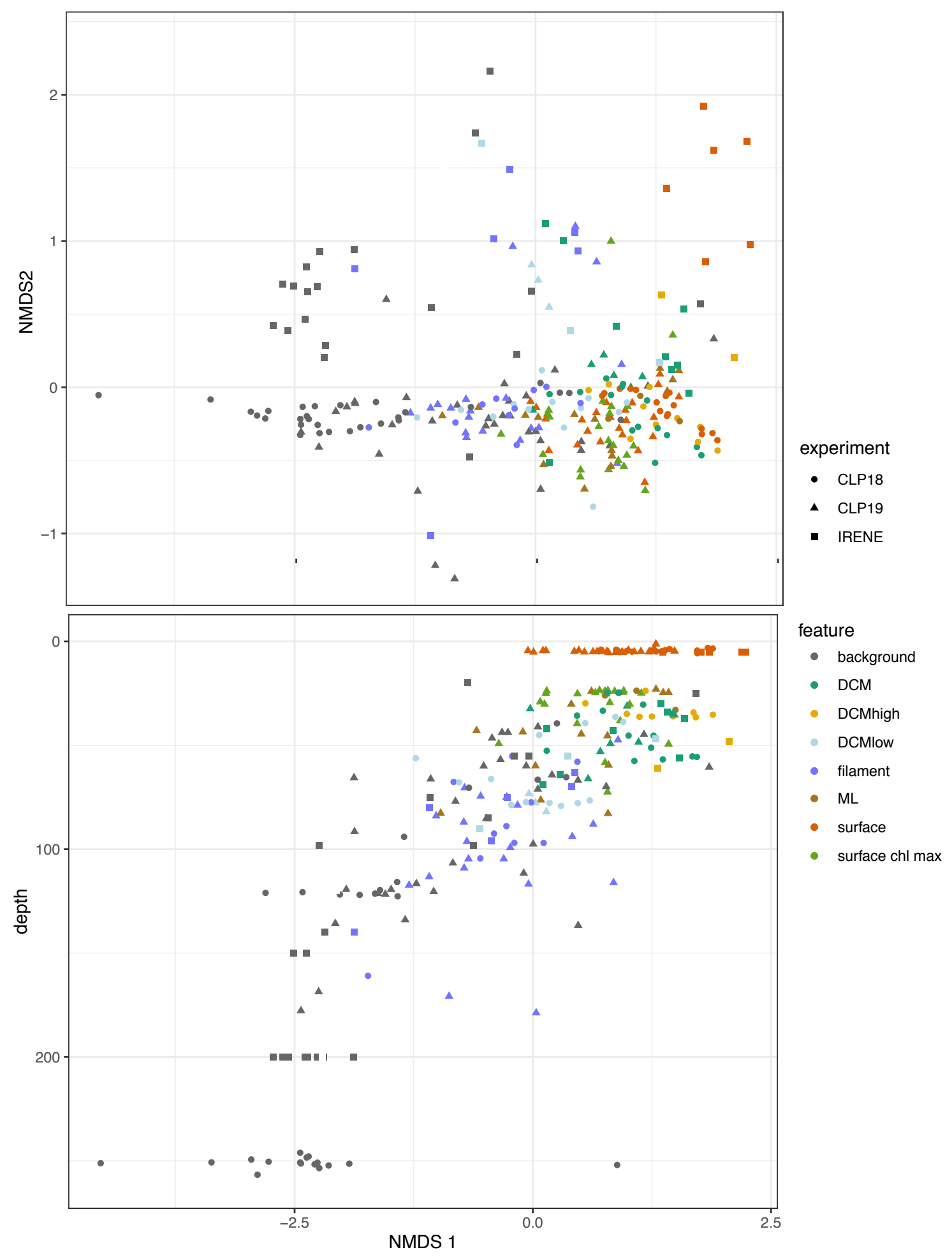

Figure F-8: Ordination of cyanobacteria and viridiplantae. (top) Non-metric multidimensional scaling (NMDS) of the Bray-Curtis distance between samples. The sample feature is shown with the color of the point and the research cruise is shown in the shape of the point. (bottom) The first NMDS axis is plotted against the sample depth. 


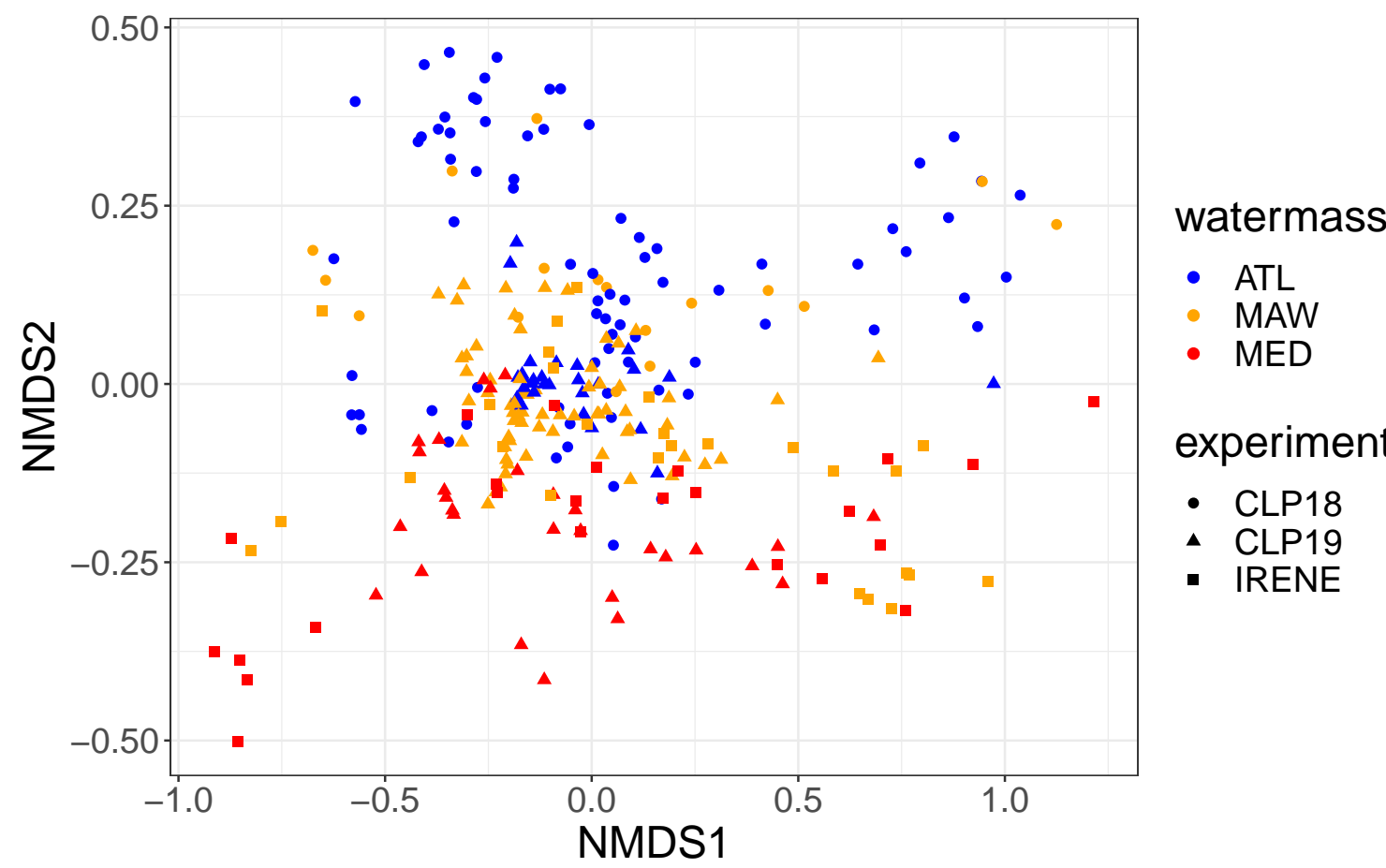

Figure F-9: NMDS ordination of Bray-Curtis dissimilarity between samples computed on ASVs (excluding plastids and cyanobacteria). The sample water mass assigned based on $\mathrm{T}$-S characteristics is shown with the color of the point and the research cruise is shown in the shape of the point. NMDS stress $=0.071$ with 3 axes. 


\section{Bibliography}

Abraham, E. R. (1998). The generation of plankton patchiness by turbulent stirring. Nature, 391(6667):577-580.

Agogué, H., Lamy, D., Neal, P. R., Sogin, M. L., and Herndl, G. J. (2011). Water massspecificity of bacterial communities in the North Atlantic revealed by massively parallel sequencing. Molecular Ecology, 20(2):258-274.

Allen, J. T., Smeed, D., Tintoré, J., and Ruiz, S. (2001). Mesoscale subduction at the Almeria-Oran front: Part 1: Ageostrophic flow. Journal of Marine Systems, 30(3-4):263285 .

Alonso-González, I. J., Arístegui, J., Vilas, J. C., and Hernández-Guerra, A. (2009). Lateral POC transport and consumption in surface and deep waters of the Canary Current region: A box model study. Global Biogeochemical Cycles, 23(2).

Alou-Font, E., Roy, S., Agustí, S., and Gosselin, M. (2016). Cell viability, pigments and photosynthetic performance of Arctic phytoplankton in contrasting ice-covered and openwater conditions during the spring-summer transition. Marine Ecology Progress Series, 543:89-106.

Alvain, S., Moulin, C., Dandonneau, Y., and Loisel, H. (2008). Seasonal distribution and succession of dominant phytoplankton groups in the global ocean: A satellite view. Global Biogeochemical Cycles, 22(3).

Archer, M., Schaeffer, A., Keating, S., Roughan, M., Holmes, R., and Siegelman, L. (2020). Observations of submesoscale variability and frontal subduction within the mesoscale eddy field of the Tasman Sea. Journal of Physical Oceanography, 50(5):1509-1529.

Ascani, F., Richards, K. J., Firing, E., Grant, S., Johnson, K. S., Jia, Y., Lukas, R., and Karl, D. M. (2013). Physical and biological controls of nitrate concentrations in the upper subtropical North Pacific ocean. Deep Sea Research Part II: Topical Studies in Oceanography, 93:119-134.

Badin, G., Tandon, A., and Mahadevan, A. (2011). Lateral mixing in the pycnocline by baroclinic mixed layer eddies. Journal of Physical Oceanography, 41(11):2080-2101.

Baltar, F., Arístegui, J., Gasol, J. M., and Herndl, G. J. (2012). Microbial Functioning and Community Structure Variability in the Mesopelagic and Epipelagic Waters of the Subtropical Northeast Atlantic Ocean. Applied and Environmental Microbiology, 78(9):33093316 . 
Balwada, D., Smith, K. S., and Abernathey, R. (2018). Submesoscale vertical velocities enhance tracer subduction in an idealized Antarctic Circumpolar Current. Geophysical Research Letters, 45(18):9790-9802.

Barkan, R., Molemaker, M. J., Srinivasan, K., McWilliams, J. C., and D'Asaro, E. A. (2019). The role of horizontal divergence in submesoscale frontogenesis. Journal of Physical Oceanography, 49(6):1593-1618.

Barone, B., Coenen, A. R., Beckett, S. J., McGillicuddy, D. J., Weitz, J. S., and Karl, D. M. (2019). The ecological and biogeochemical state of the North Pacific Subtropical Gyre is linked to sea surface height. Journal of Marine Research, 77(2):215-245.

Barth, J. A., Cowles, T. J., Kosro, P. M., Shearman, R. K., Huyer, A., and Smith, R. L. (2002). Injection of carbon from the shelf to offshore beneath the euphotic zone in the California Current. Journal of Geophysical Research: Oceans, 107(C6):10-1.

Beaird, N., Rhines, P., and Eriksen, C. (2016). Observations of seasonal subduction at the Iceland-Faroe front. Journal of Geophysical Research: Oceans, 121(6):4026-4040.

Behrenfeld, M. J., O’Malley, R. T., Siegel, D. A., McClain, C. R., Sarmiento, J. L., Feldman, G. C., Milligan, A. J., Falkowski, P. G., Letelier, R. M., and Boss, E. S. (2006). Climatedriven trends in contemporary ocean productivity. Nature, 444(7120):752-755.

Boccaletti, G., Ferrari, R., and Fox-Kemper, B. (2007). Mixed layer instabilities and restratification. Journal of Physical Oceanography, 37(9):2228-2250.

Bodner, A. S., Fox-Kemper, B., Van Roekel, L. P., McWilliams, J. C., and Sullivan, P. P. (2020). A perturbation approach to understanding the effects of turbulence on frontogenesis. Journal of Fluid Mechanics, 883.

Bokulich, N. A., Kaehler, B. D., Rideout, J. R., Dillon, M., Bolyen, E., Knight, R., Huttley, G. A., and Caporaso, J. G. (2018). Optimizing taxonomic classification of marker-gene amplicon sequences with QIIME 2's q2-feature-classifier plugin. Microbiome, 6(1):1-17.

Bolaños, L. M., Karp-Boss, L., Choi, C. J., Worden, A. Z., Graff, J. R., Haëntjens, N., Chase, A. P., Della Penna, A., Gaube, P., Morison, F., Menden-Deuer, S., Westberry, T. K., O'Malley, R. T., Boss, E., Behrenfeld, M. J., and Giovannoni, S. J. (2020). Small phytoplankton dominate western North Atlantic biomass. The ISME Journal, 14(7):16631674 .

Bosse, A., Testor, P., Mortier, L., Prieur, L., Taillandier, V., d'Ortenzio, F., and Coppola, L. (2015). Spreading of Levantine Intermediate Waters by submesoscale coherent vortices in the northwestern Mediterranean Sea as observed with gliders. Journal of Geophysical Research: Oceans, 120(3):1599-1622.

Bower, A. S. (1991). A simple kinematic mechanism for mixing fluid parcels across a meandering jet. Journal of Physical Oceanography, 21(1):173-180.

Bower, A. S., Rossby, H. T., and Lillibridge, J. L. (1985). The Gulf Stream-barrier or blender? Journal of Physical Oceanography, 15(1):24-32. 
Brett, G. J., Pratt, L. J., Rypina, I. I., and Sánchez-Garrido, J. C. (2020). The Western Alboran Gyre: An analysis of its properties and its exchange with surrounding water. Journal of Physical Oceanography, 50(12):3379-3402.

Brett, G. J., Whitt, D. B., Long, M. C., Bryan, F., Feloy, K., and Richards, K. J. (2021). Sensitivity of 21st-century projected ocean new production changes to idealized biogeochemical model structure. Biogeosciences, 18:3123-3145.

Buckingham, C. E., Gula, J., and Carton, X. (2021). The role of curvature in modifying frontal instabilities. part I: Review of theory and presentation of a nondimensional instability criterion. Journal of Physical Oceanography, 51(2):299-315.

Buitenhuis, E. T., Li, W. K., Vaulot, D., Lomas, M. W., Landry, M., Partensky, F., Karl, D., Ulloa, O., Campbell, L., Jacquet, S., Lantoine, F., Chavez, F., Macias, D., Gosselin, M., and McManus, G. B. (2012). Picophytoplankton biomass distribution in the global ocean. Earth System Science Data, 4(1):37-46.

Cael, B. B. and Bisson, K. (2018). Particle Flux Parameterizations: Quantitative and Mechanistic Similarities and Differences. Frontiers in Marine Science, 5.

Caldwell, D. R. (1983). Small-scale physics of the ocean. Reviews of Geophysics, 21(5):11921205.

Calil, P. and Richards, K. (2010). Transient upwelling hot spots in the oligotrophic North Pacific. Journal of Geophysical Research: Oceans, 115(C2).

Callies, J., Barkan, R., and Garabato, A. N. (2020). Time scales of submesoscale flow inferred from a mooring array. Journal of Physical Oceanography, 50(4):1065-1086.

Callies, J. and Ferrari, R. (2013). Interpreting energy and tracer spectra of upper-ocean turbulence in the submesoscale range $(1-200 \mathrm{~km})$. Journal of Physical Oceanography, $43(11): 2456-2474$.

Callies, J., Flierl, G., Ferrari, R., and Fox-Kemper, B. (2016). The role of mixed-layer instabilities in submesoscale turbulence. Journal of Fluid Mechanics, 788:5-41.

Canuto, V., Cheng, Y., and Howard, A. (2018). Subduction by submesoscales. Journal of Geophysical Research: Oceans, 123(12):8688-8700.

Capet, X., McWilliams, J. C., Molemaker, M. J., and Shchepetkin, A. (2008). Mesoscale to submesoscale transition in the California Current System. Part I: Flow structure, eddy flux, and observational tests. Journal of Physical Oceanography, 38(1):29-43.

Carlson, C. A., Morris, R., Parsons, R., Treusch, A. H., Giovannoni, S. J., and Vergin, K. (2009). Seasonal dynamics of SAR11 populations in the euphotic and mesopelagic zones of the northwestern sargasso sea. The ISME Journal, 3(3):283-295.

Charria, N. G., Mélin, F., Dadou, I., Radenac, M.-H., and Garçon, V. (2003). Rossby wave and ocean color: The cells uplifting hypothesis in the South Atlantic Subtropical Convergence Zone. Geophysical Research Letters, 30(3).

Chen, H. and McKinley, G. A. (2019). Isopycnal Processes Allow for Summertime Heterotrophy Despite Net Oxygen Accumulation in the Lower Euphotic Zone of the Western North Atlantic Subtropical Gyre. Global Biogeochemical Cycles, 33(6):795-809. 
Chen, S. and Qiu, B. (2010). Mesoscale eddies northeast of the Hawaiian archipelago from satellite altimeter observations. Journal of Geophysical Research: Oceans, 115(C3).

Choi, C. J., Bachy, C., Jaeger, G. S., Poirier, C., Sudek, L., Sarma, V., Mahadevan, A., Giovannoni, S. J., and Worden, A. Z. (2017). Newly discovered deep-branching marine plastid lineages are numerically rare but globally distributed. Current Biology, 27(1):R15R16.

Choi, C. J., Jimenez, V., Needham, D., Poirier, C., Bachy, C., Alexander, H., Wilken, S., Chavez, F., Sudek, S., Giovannoni, S. J., and Worden, A. Z. (2020). Seasonal and geographical transitions in eukaryotic phytoplankton community structure in the Atlantic and Pacific Oceans. Frontiers in microbiology, 11:2187.

Church, M. J., Ducklow, H. W., Letelier, R. M., and Karl, D. M. (2006). Temporal and vertical dynamics in picoplankton photoheterotrophic production in the subtropical North Pacific ocean. Aquatic Microbial Ecology, 45(1):41-53.

Claustre, H., Kerhervé, P., Marty, J. C., Prieur, L., Videau, C., and Hecq, J.-H. (1994). Phytoplankton dynamics associated with a geostrophic front: ecological and biogeochemical implications. Journal of Marine Research, 52(4):711-742.

Clayton, S., Dutkiewicz, S., Jahn, O., and Follows, M. J. (2013). Dispersal, eddies, and the diversity of marine phytoplankton. Limnology and Oceanography: Fluids and Environments, 3(1):182-197.

Clayton, S., Nagai, T., and Follows, M. J. (2014). Fine scale phytoplankton community structure across the Kuroshio Front. Journal of Plankton Research, 36(4):1017-1030.

Cole, S. T. and Rudnick, D. L. (2012). The spatial distribution and annual cycle of upper ocean thermohaline structure. Journal of Geophysical Research: Oceans, 117(C2).

Cole, S. T., Wortham, C., Kunze, E., and Owens, W. B. (2015). Eddy stirring and horizontal diffusivity from Argo float observations: Geographic and depth variability. Geophysical Research Letters, 42(10):3989-3997.

Collins, J. R., Fucile, P. D., McDonald, G., Ossolinski, J. E., Keil, R. G., Valdes, J. R., Doney, S. C., and Van Mooy, B. A. (2018). An autonomous, in situ light-dark bottle device for determining community respiration and net community production. Limnology and Oceanography: Methods, 16(6):323-338.

Cram, J. A., Chow, C.-E. T., Sachdeva, R., Needham, D. M., Parada, A. E., Steele, J. A., and Fuhrman, J. A. (2015). Seasonal and interannual variability of the marine bacterioplankton community throughout the water column over ten years. The ISME Journal, $9(3): 563-580$.

Cullen, J. J. (2015). Subsurface chlorophyll maximum layers: enduring enigma or mystery solved? Annual Review of Marine Science, 7:207-239.

Dall'Olmo, G., Dingle, J., Polimene, L., Brewin, R. J. W., and Claustre, H. (2016). Substantial energy input to the mesopelagic ecosystem from the seasonal mixed-layer pump. Nature Geoscience, 9(11):820-823. 
D'Asaro, E. A. (2001). Turbulent vertical kinetic energy in the ocean mixed layer. Journal of Physical Oceanography, 31(12):3530-3537.

D'Asaro, E. A. (2003). Performance of autonomous Lagrangian floats. Journal of Atmospheric and Oceanic Technology, 20(6):896-911.

D'Asaro, E. A., Shcherbina, A. Y., Klymak, J. M., Molemaker, J., Novelli, G., Guigand, C. M., Haza, A. C., Haus, B. K., Ryan, E. H., Jacobs, G. A., Huntley, H. S., Laxague, N. J., Chen, S., Judt, F., McWilliams, J. C., Barkan, R., Kirwan Jr., A. D., Poje, A. C., and Özgökmen, T. (2018). Ocean convergence and the dispersion of flotsam. Proceedings of the National Academy of Sciences, 115(6):1162-1167.

DeLong, E. F., Preston, C. M., Mincer, T., Rich, V., Hallam, S. J., Frigaard, N.-U., Martinez, A., Sullivan, M. B., Edwards, R., Brito, B. R., et al. (2006). Community genomics among stratified microbial assemblages in the ocean's interior. Science, 311(5760):496503.

Demir-Hilton, E., Sudek, S., Cuvelier, M. L., Gentemann, C. L., Zehr, J. P., and Worden, A. Z. (2011). Global distribution patterns of distinct clades of the photosynthetic picoeukaryote ostreococcus. The ISME Journal, 5(7):1095-1107.

Denman, K. and Gargett, A. (1983). Time and space scales of vertical mixing and advection of phytoplankton in the upper ocean. Limnology and Oceanography, 28(5):801-815.

Dever, M. and Essink, S. (2020). Offline particle tracking.

Dever, M., Freilich, M., Farrar, J. T., Hodges, B., Lanagan, T., Baron, A. J., and Mahadevan, A. (2020). EcoCTD for profiling oceanic physical-biological properties from an underway ship. Journal of Atmospheric and Oceanic Technology, 37(5):825-840.

Dever, M., Freilich, M., Hodges, B. A., Farrar, J. T., Lanagan, T., and Mahadevan, A. (2019). UCTD and EcoCTD Observations from the CALYPSO Pilot Experiment (2018): Cruise and Data Report. Woods Hole Oceanographic Institution.

Dever, M., Nicholson, D., Omand, M., and Mahadevan, A. (2021). Size-differentiated export flux in different dynamical regimes in the ocean. Global Biogeochemical Cycles, 35(3):e2020GB006764.

Dickey, T., Zedler, S., Yu, X., Doney, S., Frye, D., Jannasch, H., Manov, D., Sigurdson, D., McNeil, J., Dobeck, L., et al. (2001). Physical and biogeochemical variability from hours to years at the Bermuda Testbed Mooring site: June 1994-March 1998. Deep Sea Research Part II: Topical Studies in Oceanography, 48(8):2105-2140.

Doddridge, E. W., Marshall, D. P., and Hogg, A. M. (2016). Eddy cancellation of the Ekman cell in subtropical gyres. Journal of Physical Oceanography, 46(10):2995-3010.

d'Ovidio, F., De Monte, S., Alvain, S., Dandonneau, Y., and Lévy, M. (2010). Fluid dynamical niches of phytoplankton types. Proceedings of the National Academy of Sciences, 107(43):18366-18370.

Ducklow, H. W. and Harris, R. P. (1993). Introduction to the JGOFS North Atlantic bloom experiment. Deep Sea Research Part II: Topical Studies in Oceanography, 40(1-2):1-8. 
Ducklow, H. W., Steinberg, D. K., and Buesseler, K. O. (2001). Upper ocean carbon export and the biological pump. Oceanography, 14(4):50-58.

Dusenberry, J., Olson, R., and Chisholm, S. (2000). Field observations of oceanic mixed layer dynamics and picophytoplankton photoacclimation. Journal of Marine Systems, 24(3-4):221-232.

Edgar, R. C. and Flyvbjerg, H. (2015). Error filtering, pair assembly and error correction for next-generation sequencing reads. Bioinformatics, 31(21):3476-3482.

Eliassen, A. (1949). The quasi-static equations of motion with pressure as independent variable. Grøndahl \& Sons boktr., I kommisjon hos Cammermeyers boghandel.

Emerson, S. (2014). Annual net community production and the biological carbon flux in the ocean. Global Biogeochemical Cycles, 28(1):14-28.

Erickson, Z. K. and Thompson, A. F. (2018). The seasonality of physically driven export at submesoscales in the northeast Atlantic Ocean. Global Biogeochemical Cycles, 32(8):11441162 .

Erickson, Z. K., Thompson, A. F., Cassar, N., Sprintall, J., and Mazloff, M. R. (2016). An advective mechanism for deep chlorophyll maxima formation in southern Drake Passage. Geophysical Research Letters, 43(20).

Eslinger, D. L. and Iverson, R. L. (1986). Wind effects on coastal zone color scanner chlorophyll patterns in the US Mid-Atlantic Bight during spring 1979. Journal of Geophysical Research: Oceans, 91(C11):12985-12992.

Estrada, M. (1996). Primary production in the northwestern Mediterranean. Scientia Marina.

Fadeev, E., Wietz, M., von Appen, W.-J., Iversen, M. H., Nöthig, E.-M., Engel, A., Grosse, J., Graeve, M., and Boetius, A. (2021). Submesoscale physicochemical dynamics directly shape bacterioplankton community structure in space and time. Limnology and Oceanography.

Falkowski, P. G., Barber, R. T., and Smetacek, V. (1998). Biogeochemical controls and feedbacks on ocean primary production. Science, 281(5374):200-206.

Ferrari, R. and Rudnick, D. L. (2000). Thermohaline variability in the upper ocean. Journal of Geophysical Research: Oceans, 105(C7):16857-16883.

Ferrón, S., Barone, B., Church, M. J., White, A. E., and Karl, D. M. (2021). Euphotic zone metabolism in the North Pacific Subtropical Gyre based on oxygen dynamics. Global Biogeochemical Cycles, 35(3):e2020GB006744.

Fielding, S., Crisp, N., Allen, J., Hartman, M., Rabe, B., and Roe, H. (2001). Mesoscale subduction at the Almeria-Oran front: part 2. Biophysical interactions. Journal of Marine Systems, 30(3):287-304.

Finkel, Z. V., Beardall, J., Flynn, K. J., Quigg, A., Rees, T. A. V., and Raven, J. A. (2010). Phytoplankton in a changing world: cell size and elemental stoichiometry. Journal of plankton research, 32(1):119-137. 
Flierl, G. and McGillicuddy, D. J. (2002). Mesoscale and submesoscale physical-biological interactions. The Sea, 12:113-185.

Flombaum, P., Gallegos, J. L., Gordillo, R. A., Rincón, J., Zabala, L. L., Jiao, N., Karl, D. M., Li, W. K., Lomas, M. W., Veneziano, D., et al. (2013). Present and future global distributions of the marine cyanobacteria Prochlorococcus and Synechococcus. Proceedings of the National Academy of Sciences, 110(24):9824-9829.

Fontanez, K. M., Eppley, J. M., Samo, T. J., Karl, D. M., and DeLong, E. F. (2015). Microbial community structure and function on sinking particles in the North Pacific Subtropical Gyre. Frontiers in Microbiology, 6.

Fox-Kemper, B., Ferrari, R., and Hallberg, R. (2008). Parameterization of mixed layer eddies. Part I: Theory and diagnosis. Journal of Physical Oceanography, 38(6):1145-1165.

Frajka-Williams, E., Eriksen, C. C., Rhines, P. B., and Harcourt, R. R. (2011). Determining vertical water velocities from seaglider. Journal of Atmospheric and Oceanic Technology, 28(12):1641-1656.

Franks, P. J. (2001). Phytoplankton blooms in a fluctuating environment: the roles of plankton response time scales and grazing. Journal of plankton research, 23(12):14331441.

Freilich, M. (2018). Forecasting where ocean life thrives: Scientists focus on seams in the ocean called'fronts'. Oceanus, 53(2):20-24.

Freilich, M. and Mahadevan, A. (2021). Coherent pathways for subduction from the surface mixed layer at ocean fronts. Journal of Geophysical Research: Oceans, page e2020JC017042.

Freilich, M. A. and Mahadevan, A. (2019). Decomposition of vertical velocity for nutrient transport in the upper ocean. Journal of Physical Oceanography, 49(6):1561-1575.

Frenger, I., Bianchi, D., Oschlies, A., and Waschkowitz, C. (2017). Subsurface coherent eddies: Hypoxic stewpots and biogeochemical highways. EGUGA, page 2236.

Fuenzalida, H. A., Sánchez, R., and Garreaud, R. D. (2005). A climatology of cutoff lows in the Southern Hemisphere. Journal of Geophysical Research: Atmospheres, 110(D18).

Gebbie, G. (2007). Does eddy subduction matter in the northeast Atlantic Ocean? Journal of Geophysical Research: Oceans, 112(C6).

Gent, P. R. and Mcwilliams, J. C. (1990). Isopycnal mixing in ocean circulation models. Journal of Physical Oceanography, 20(1):150-155.

Gernez, P., Antoine, D., and Huot, Y. (2011). Diel cycles of the particulate beam attenuation coefficient under varying trophic conditions in the northwestern Mediterranean Sea: Observations and modeling. Limnology and Oceanography, 56(1):17-36.

Giering, S. L., Sanders, R., Lampitt, R. S., Anderson, T. R., Tamburini, C., Boutrif, M., Zubkov, M. V., Marsay, C. M., Henson, S. A., Saw, K., Cook, K., and Mayor, D. J. (2014). Reconciliation of the carbon budget in the ocean's twilight zone. Nature, 507(7493):480483. 
Giordani, H., Prieur, L., and Caniaux, G. (2006). Advanced insights into sources of vertical velocity in the ocean. Ocean Dynamics, 56(5-6):513-524.

Giovannoni, S. J. and Vergin, K. L. (2012). Seasonality in ocean microbial communities. Science, 335(6069):671-676.

Gnanadesikan, A., Pradal, M.-A., and Abernathey, R. (2015). Isopycnal mixing by mesoscale eddies significantly impacts oceanic anthropogenic carbon uptake. Geophysical Research Letters, 42(11):4249-4255.

Goericke, R. and Welschmeyer, N. A. (1998). Response of Sargasso Sea phytoplankton biomass, growth rates and primary production to seasonally varying physical forcing. Journal of Plankton Research, 20(12):2223-2249.

Gorsky, G., Prieur, L., Taupier-Letage, I., Stemmann, L., and Picheral, M. (2002). Large particulate matter in the Western Mediterranean: I. LPM distribution related to mesoscale hydrodynamics. Journal of Marine Systems, 33:289-311.

Gruber, N., Lachkar, Z., Frenzel, H., Marchesiello, P., Münnich, M., McWilliams, J. C., Nagai, T., and Plattner, G.-K. (2011). Eddy-induced reduction of biological production in eastern boundary upwelling systems. Nature Geoscience, 4(11):787-792.

Guidi, L., Calil, P. H., Duhamel, S., Björkman, K. M., Doney, S. C., Jackson, G. A., Li, B., Church, M. J., Tozzi, S., Kolber, Z. S., Richards, K., Fong, A. A., Letelier, R., Gorsky, G., Stemmann, L., and Karl, D. M. (2012). Does eddy-eddy interaction control surface phytoplankton distribution and carbon export in the North Pacific subtropical gyre? Journal of Geophysical Research: Biogeosciences, 117(G2).

Guidi, L., Chaffron, S., Bittner, L., Eveillard, D., Larhlimi, A., Roux, S., Darzi, Y., Audic, S., Berline, L., Brum, J. R., Coelho, L. P., Espinoza, J. C. I., Malviya, S., Sunagawa, S., Dimier, C., Kandels-Lewis, S., Picheral, M., Poulain, J., Searson, S., Stemmann, L., Not, F., Hingamp, P., Speich, S., Follows, M., Karp-Boss, L., Boss, E., Ogata, H., Pesant, S., Weissenbach, J., Wincker, P., Acinas, S. G., Bork, P., de Vargas, C., Iudicone, D., Sullivan, M. B., Raes, J., Karsenti, E., Bowler, C., and Gorsky, G. (2016). Plankton networks driving carbon export in the oligotrophic ocean. Nature, 532(7600):465-470.

Gula, J., Molemaker, M. J., and McWilliams, J. C. (2014). Submesoscale cold filaments in the Gulf Stream. Journal of Physical Oceanography, 44(10):2617-2643.

Gula, J., Molemaker, M. J., and McWilliams, J. C. (2016). Submesoscale dynamics of a Gulf Stream frontal eddy in the South Atlantic Bight. Journal of Physical Oceanography, 46(1):305-325.

Haine, T. W. and Marshall, J. (1998). Gravitational, symmetric, and baroclinic instability of the ocean mixed layer. Journal of Physical Oceanography, 28(4):634-658.

Henson, S. A., Sanders, R., Madsen, E., Morris, P. J., Le Moigne, F., and Quartly, G. D. (2011). A reduced estimate of the strength of the ocean's biological carbon pump. Geophysical Research Letters, 38(4).

Hodges, B. A. and Rudnick, D. L. (2004). Simple models of steady deep maxima in chlorophyll and biomass. Deep Sea Research Part I: Oceanographic Research Papers, 51(8):9991015 . 
Holmes, R. M., Thomas, L. N., Thompson, L., and Darr, D. (2014). Potential vorticity dynamics of tropical instability vortices. Journal of Physical Oceanography, 44(3):9951011.

Holton, J. R., Haynes, P. H., McIntyre, M. E., Douglass, A. R., Rood, R. B., and Pfister, L. (1995). Stratosphere-troposphere exchange. Reviews of Geophysics, 33(4):403-439.

Hoskins, B. J. (1982). The mathematical theory of frontogenesis. Annual Review of Fluid Mechanics, 14(1):131-151.

Hoskins, B. J. and Bretherton, F. P. (1972). Atmospheric frontogenesis models: Mathematical formulation and solution. Journal of the Atmospheric Sciences, 29(1):11-37.

Hoskins, B. J., Draghici, I., and Davies, H. (1978). A new look at the w-equation. Quarterly Journal of the Royal Meteorological Society, 104(439):31-38.

Hoskins, B. J. and James, I. N. (2014). Fluid dynamics of the mid-latitude atmosphere. John Wiley \& Sons.

Jenkins, W. and Goldman, J. (1985). Seasonal oxygen cycling and primary production in the Sargasso Sea. Journal of Marine Research, 43(2):465-491.

Jin, X., Najjar, R., Louanchi, F., and Doney, S. C. (2007). A modeling study of the seasonal oxygen budget of the global ocean. Journal of Geophysical Research: Oceans, 112(C5).

Johnson, A. R. and Omand, M. M. (2021). Evolution of a subducted carbon-rich filament on the edge of the North Atlantic Gyre. Journal of Geophysical Research: Oceans, 26(2):e2020JC016685.

Johnson, K. S., Riser, S. C., and Karl, D. M. (2010). Nitrate supply from deep to near-surface waters of the North Pacific subtropical gyre. Nature, 465(7301):1062-1065.

Johnson, L., Lee, C. M., and D'Asaro, E. A. (2016). Global Estimates of Lateral Springtime Restratification. Journal of Physical Oceanography, 46(5):1555-1573.

Johnston, T. S., MacKinnon, J. A., Colin, P. L., Haley, P. J., Lermusiaux, P. F., Lucas, A. J., Merrifield, M. A., Merrifield, S. T., Mirabito, C., Nash, J. D., Ou, C. Y., Siegelman, M., Terrill, E. J., and Waterhouse, A. F. (2019). Energy and momentum lost to wake eddies and lee waves generated by the North Equatorial Current and tidal flows at Peleliu, Palau. Oceanography, 32(4):110-125.

Joshi, N. and Fass, J. (2011). Sickle: A sliding-window, adaptive, quality-based trimming tool for fastq files (version 1.33)[software].

Kadko, D. C., Washburn, L., and Jones, B. (1991). Evidence of subduction within cold filaments of the northern California coastal transition zone. Journal of Geophysical Research: Oceans, 96(C8):14909-14926.

Kahru, M. (1983). Phytoplankton patchiness generated by long internal waves: A model. Marine ecology progress series. Oldendorf, 10(2):111-117.

Keeling, C. D., Brix, H., and Gruber, N. (2004). Seasonal and long-term dynamics of the upper ocean carbon cycle at Station ALOHA near Hawaii. Global Biogeochemical Cycles, $18(4)$. 
Kheireddine, M. and Antoine, D. (2014). Diel variability of the beam attenuation and backscattering coefficients in the northwestern Mediterranean Sea (BOUSSOLE site). Journal of Geophysical Research: Oceans, 119(8):5465-5482.

Klein, P. and Lapeyre, G. (2009). The oceanic vertical pump induced by mesoscale and submesoscale turbulence. Annual Review of Marine Science, 1(1):351-375.

Klymak, J. M., Crawford, W., Alford, M. H., MacKinnon, J. A., and Pinkel, R. (2015). Along-isopycnal variability of spice in the North Pacific. Journal of Geophysical Research: Oceans, 120(3):2287-2307.

Klymak, J. M., Shearman, R. K., Gula, J., Lee, C. M., D'Asaro, E. A., Thomas, L. N., Harcourt, R. R., Shcherbina, A. Y., Sundermeyer, M. A., Molemaker, J., and McWilliams, J. C. (2016). Submesoscale streamers exchange water on the north wall of the Gulf Stream. Geophysical Research Letters, 43(3):1226-1233.

Kunze, E., Klymak, J., Lien, R.-C., Ferrari, R., Lee, C., Sundermeyer, M., and Goodman, L. (2015). Submesoscale water-mass spectra in the Sargasso Sea. Journal of Physical Oceanography, 45(5):1325-1338.

Kwon, E. Y., Primeau, F., and Sarmiento, J. L. (2009). The impact of remineralization depth on the air-sea carbon balance. Nature Geoscience, 2(9):630-635.

Lathuiliere, C., Levy, M., and Echevin, V. (2010). Impact of eddy-driven vertical fluxes on phytoplankton abundance in the euphotic layer. Journal of Plankton Research.

Laws, E. A. (2013). Evaluation of in situ phytoplankton growth rates: a synthesis of data from varied approaches. Annual Review of Marine Science, 5:247-268.

Ledwell, J. R., Watson, A. J., and Law, C. S. (1993). Evidence for slow mixing across the pycnocline from an open-ocean tracer-release experiment. Nature, 364(6439):701.

Lee, S. and Fuhrman, J. A. (1987). Relationships between biovolume and biomass of naturally derived marine bacterioplankton. Appl. Environ. Microbiol., 53(6):1298-1303.

Letelier, R. M., Karl, D. M., Abbott, M. R., Flament, P., Freilich, M., Lukas, R., and Strub, T. (2000). Role of late winter mesoscale events in the biogeochemical variability of the upper water column of the North Pacific subtropical gyre. Journal of Geophysical Research: Oceans, 105(C12):28723-28739.

Lévy, M., Bopp, L., Karleskind, P., Resplandy, L., Éthé, C., and Pinsard, F. (2013). Physical pathways for carbon transfers between the surface mixed layer and the ocean interior. Global Biogeochemical Cycles, 27(4):1001-1012.

Lévy, M., Ferrari, R., Franks, P. J., Martin, A. P., and Rivière, P. (2012a). Bringing physics to life at the submesoscale. Geophysical Research Letters, 39(14).

Lévy, M., Franks, P. J., and Smith, K. S. (2018). The role of submesoscale currents in structuring marine ecosystems. Nature Communications, 9(1):1-16.

Lévy, M., Iovino, D., Resplandy, L., Klein, P., Madec, G., Tréguier, A.-M., Masson, S., and Takahashi, K. (2012b). Large-scale impacts of submesoscale dynamics on phytoplankton: Local and remote effects. Ocean Modelling, 43:77-93. 
Lévy, M., Jahn, O., Dutkiewicz, S., Follows, M. J., and d'Ovidio, F. (2015). The dynamical landscape of marine phytoplankton diversity. Journal of The Royal Society Interface, 12(111):20150481.

Lévy, M., Klein, P., and Treguier, A.-M. (2001). Impact of sub-mesoscale physics on production and subduction of phytoplankton in an oligotrophic regime. Journal of Marine Research, 59(4):535-565.

Lévy, M., Resplandy, L., Klein, P., Capet, X., Iovino, D., and Éthé, C. (2012c). Grid degradation of submesoscale resolving ocean models: Benefits for offline passive tracer transport. Ocean Modelling, 48:1-9.

Lewis, M. R., Hebert, D., Harrison, W. G., Platt, T., and Oakey, N. S. (1986). Vertical nitrate fluxes in the oligotrophic ocean. Science, 234(4778):870-873.

Lipschultz, F., Bates, N. R., Carlson, C. A., and Hansell, D. A. (2002). New production in the Sargasso Sea: History and current status. Global Biogeochemical Cycles, 16(1):1-1.

Liu, X. and Levine, N. M. (2021). Ecosystem implications of fine-scale frontal disturbances in the oligotrophic ocean - an idealized modeling approach. Progress in Oceanography, page 102519.

Llanillo, P., Pelegrí, J. L., Talley, L., Peña-Izquierdo, J., and Cordero, R. (2018). Oxygen pathways and budget for the eastern South Pacific oxygen minimum zone. Journal of Geophysical Research: Oceans, 123(3):1722-1744.

Llort, J., Langlais, C., Matear, R., Moreau, S., Lenton, A., and Strutton, P. G. (2018). Evaluating Southern Ocean Carbon Eddy-Pump From Biogeochemical-Argo Floats. Journal of Geophysical Research: Oceans, 123(2):971-984.

Lohrenz, S. E., Wiesenburg, D. A., DePalma, I. P., Johnson, K. S., and Gustafson Jr, D. E. (1988). Interrelationships among primary production, chlorophyll, and environmental conditions in frontal regions of the western Mediterranean Sea. Deep Sea Research Part A. Oceanographic Research Papers, 35(5):793-810.

Lomas, M. W. and Moran, S. B. (2011). Evidence for aggregation and export of cyanobacteria and nano-eukaryotes from the Sargasso Sea euphotic zone. Biogeosciences, 8(1):203216.

López, C., Neufeld, Z., Hernandez-Garcia, E., and Haynes, P. H. (2001). Chaotic advection of reacting substances: Plankton dynamics on a meandering jet. Physics and Chemistry of the Earth, Part B: Hydrology, Oceans and Atmosphere, 26(4):313-317.

Löptien, U. and Dietze, H. (2019). Reciprocal bias compensation and ensuing uncertainties in model-based climate projections: pelagic biogeochemistry versus ocean mixing. Biogeosciences, 16(9):1865-1881.

Lozier, M. S., Bacon, S., Bower, A. S., Cunningham, S. A., De Jong, M. F., De Steur, L., Deyoung, B., Fischer, J., Gary, S. F., Greenan, B. J., et al. (2017). Overturning in the subpolar North Atlantic program: A new international ocean observing system. Bulletin of the American Meteorological Society, 98(4):737-752. 
MacGilchrist, G. A., Marshall, D. P., Johnson, H. L., Lique, C., and Thomas, M. (2017). Characterizing the chaotic nature of ocean ventilation. Journal of Geophysical Research: Oceans, 122(9):7577-7594.

Mahadevan, A. (2016). The impact of submesoscale physics on primary productivity of plankton. Annual Review of Marine Science, 8:161-184.

Mahadevan, A. (2020). PSOM.

Mahadevan, A. and Archer, D. (2000). Modeling the impact of fronts and mesoscale circulation on the nutrient supply and biogeochemistry of the upper ocean. Journal of Geophysical Research. C. Oceans, 105:1209-1225.

Mahadevan, A. and Campbell, J. (2002). Biogeochemical patchiness at the sea surface. Geophysical Research Letters, 29(19).

Mahadevan, A., D'asaro, E., Lee, C., and Perry, M. J. (2012). Eddy-driven stratification initiates North Atlantic spring phytoplankton blooms. Science, 337(6090):54-58.

Mahadevan, A., Jaeger, G. S., Freilich, M., Omand, M. M., Shroyer, E. L., and Sengupta, D. (2016). Freshwater in the Bay of Bengal: Its fate and role in air-sea heat exchange. Oceanography, 29(2):72-81.

Mahadevan, A., Oliger, J., and Street, R. (1996a). A nonhydrostatic mesoscale ocean model. Part I: Well-posedness and scaling. Journal of Physical Oceanography, 26(9):1868-1880.

Mahadevan, A., Oliger, J., and Street, R. (1996b). A nonhydrostatic mesoscale ocean model. part i: Well-posedness and scaling. Journal of Physical Oceanography, 26(9):1868-1880.

Mahadevan, A., Oliger, J., and Street, R. (1996c). A nonhydrostatic mesoscale ocean model. Part II: Numerical implementation. Journal of Physical Oceanography, 26(9):1881-1900.

Mahadevan, A., Oliger, J., and Street, R. (1996d). A nonhydrostatic mesoscale ocean model. part ii: Numerical implementation. Journal of Physical Oceanography, 26(9):1881-1900.

Mahadevan, A., Pascual, A., Rudnick, D. L., Ruiz, S., Tintoré, J., and D'Asaro, E. (2020). Coherent pathways for vertical transport from the surface ocean to interior. Bulletin of the American Meteorological Society, 101(11):E1996-E2004.

Mahadevan, A. and Tandon, A. (2006). An analysis of mechanisms for submesoscale vertical motion at ocean fronts. Ocean Modelling, 14(3-4):241-256.

Mahadevan, A., Tandon, A., and Ferrari, R. (2010). Rapid changes in mixed layer stratification driven by submesoscale instabilities and winds. Journal of Geophysical Research: Oceans, 115(C3).

Malone, T. C., Pike, S. E., and Conley, D. J. (1993). Transient variations in phytoplankton productivity at the JGOFS Bermuda time series station. Deep Sea Research Part I: Oceanographic Research Papers, 40(5):903-924.

Mandal, S., Van Treuren, W., White, R. A., Eggesbø, M., Knight, R., and Peddada, S. D. (2015). Analysis of composition of microbiomes: a novel method for studying microbial composition. Microbial Ecology in Health and Disease, 26(1):27663. 
Marañón, E. (2015). Cell size as a key determinant of phytoplankton metabolism and community structure. Annual Review of Marine Science.

Marañón, E., Van Wambeke, F., Uitz, J., Boss, E. S., Dimier, C., Dinasquet, J., Engel, A., Haëntjens, N., Pérez-Lorenzo, M., Taillandier, V., and Zäncker, B. (2021). Deep maxima of phytoplankton biomass, primary production and bacterial production in the Mediterranean Sea. Biogeosciences, 18(5):1749-1767.

Martin, A. and Pondaven, P. (2003). On estimates for the vertical nitrate flux due to eddy pumping. Journal of Geophysical Research: Oceans (1978-2012), 108(C11).

Martin, J. H., Knauer, G. A., Karl, D. M., and Broenkow, W. W. (1987). VERTEX: carbon cycling in the northeast Pacific. Deep Sea Research Part A. Oceanographic Research Papers, 34(2):267-285.

Martin, M. (2011). Cutadapt removes adapter sequences from high-throughput sequencing reads. EMBnet. journal, 17(1):10-12.

Mayot, N., d'Ortenzio, F., Taillandier, V., Prieur, L., De Fommervault, O. P., Claustre, H., Bosse, A., Testor, P., and Conan, P. (2017). Physical and biogeochemical controls of the phytoplankton blooms in north western Mediterranean Sea: a multiplatform approach over a complete annual cycle (2012-2013 DEWEX experiment). Journal of Geophysical Research: Oceans, 122(12):9999-10019.

McDougall, T. J., Barker, P. M., and Stanley, G. J. (2021). Spice variables and their use in physical oceanography. Journal of Geophysical Research: Oceans, 126(2):e2019JC015936.

McGillicuddy, D., Robinson, A., Siegel, D., Jannasch, H., Johnson, R., Dickey, T., McNeil, J., Michaels, A., and Knap, A. (1998a). Influence of mesoscale eddies on new production in the sargasso sea. Nature, 394(6690):263-266.

McGillicuddy, D. J. (2016a). Mechanisms of physical-biological-biogeochemical interaction at the oceanic mesoscale. Annual Review of Marine Science, 8:125-159.

McGillicuddy, D. J. (2016b). Mechanisms of physical-biological-biogeochemical interaction at the oceanic mesoscale. Annual Review of Marine Science, 8:125-159.

McGillicuddy, D. J., Robinson, A. R., Siegel, D. A., Jannasch, H. W., Johnson, R., Dickey, T. D., McNeil, J., Michaels, A. F., and Knap, A. H. (1998b). Influence of mesoscale eddies on new production in the Sargasso Sea. Nature, 394(6690):263-266.

McMurdie, P. J. and Holmes, S. (2013). phyloseq: an R package for reproducible interactive analysis and graphics of microbiome census data. PloS one, 8(4):e61217.

McWilliams, J. (2021). Oceanic frontogenesis. Annual Review of Marine Science, 13:227253.

McWilliams, J., Colas, F., and Molemaker, M. (2009). Cold filamentary intensification and oceanic surface convergence lines. Geophysical Research Letters, 36(18).

McWilliams, J., Gula, J., and Molemaker, M. J. (2019). The Gulf Stream north wall: Ageostrophic circulation and frontogenesis. Journal of Physical Oceanography, 49(4):893916. 
McWilliams, J. C. (1985). Submesoscale, coherent vortices in the ocean. Reviews of Geophysics, 23(2):165-182.

McWilliams, J. C. (2016). Submesoscale currents in the ocean. Proceedings of the Royal Society A: Mathematical, Physical and Engineering Sciences, 472(2189):20160117.

Mignone, B. K., Gnanadesikan, A., Sarmiento, J. L., and Slater, R. D. (2006). Central role of Southern Hemisphere winds and eddies in modulating the oceanic uptake of anthropogenic carbon. Geophysical Research Letters, 33(1).

Miquel, J.-C., Martín, J., Gasser, B., Rodriguez-y Baena, A., Toubal, T., and Fowler, S. W. (2011). Dynamics of particle flux and carbon export in the northwestern Mediterranean Sea: A two decade time-series study at the DYFAMED site. Progress in Oceanography, 91(4):461-481.

Morán, X. A. G., Taupier-Letage, I., Vázquez-Domínguez, E., Ruiz, S., Arin, L., Raimbault, P., and Estrada, M. (2001). Physical-biological coupling in the Algerian Basin (SW Mediterranean): influence of mesoscale instabilities on the biomass and production of phytoplankton and bacterioplankton. Deep Sea Research Part I: Oceanographic Research Papers, 48(2):405-437.

Morison, F., Franzè, G., Harvey, E., and Menden-Deuer, S. (2020). Light fluctuations are key in modulating plankton trophic dynamics and their impact on primary production. Limnology and Oceanography Letters.

Mousing, E. A., Richardson, K., Bendtsen, J., Cetinić, I., and Perry, M. J. (2016). Evidence of small-scale spatial structuring of phytoplankton alpha-and beta-diversity in the open ocean. Journal of Ecology, 104(6):1682-1695.

Munk, W., Armi, L., Fischer, K., and Zachariasen, F. (2000). Spirals on the sea. Proceedings of the Royal Society of London. Series A: Mathematical, Physical and Engineering Sciences, 456(1997):1217-1280.

Needham, D. M. and Fuhrman, J. A. (2016). Pronounced daily succession of phytoplankton, archaea and bacteria following a spring bloom. Nature Microbiology, 1(4):1-7.

Nelson, C. E., Carlson, C. A., Ewart, C. S., and Halewood, E. R. (2014). Community differentiation and population enrichment of Sargasso Sea bacterioplankton in the euphotic zone of a mesoscale mode-water eddy: Bacterioplankton in a Sargasso Sea mode-water eddy. Environmental Microbiology, 16(3):871-887.

Neufeld, Z. (2012). Stirring effects in models of oceanic plankton populations. Chaos: An Interdisciplinary Journal of Nonlinear Science, 22(3):037102.

Nurser, A. G. and Marshall, J. C. (1991). On the relationship between subduction rates and diabatic forcing of the mixed layer. Journal of Physical Oceanography, 21(12):1793-1802.

Oguz, T., Macias, D., Garcia-Lafuente, J., Pascual, A., and Tintore, J. (2014). Fueling plankton production by a meandering frontal jet: a case study for the Alboran Sea (Western Mediterranean). PLoS One, 9(11):e111482.

Oka, E., Toyama, K., and Suga, T. (2009). Subduction of North Pacific central mode water associated with subsurface mesoscale eddy. Geophysical Research Letters, 36(8). 
Olita, A., Capet, A., Claret, M., Mahadevan, A., Poulain, P. M., Ribotti, A., Ruiz, S., Tintoré, J., Tovar-Sánchez, A., and Pascual, A. (2017). Frontal dynamics boost primary production in the summer stratified Mediterranean Sea. Ocean Dynamics, 67(6):767-782.

Omand, M. M., D'Asaro, E. A., Lee, C. M., Perry, M. J., Briggs, N., Cetinić, I., and Mahadevan, A. (2015). Eddy-driven subduction exports particulate organic carbon from the spring bloom. Science, 348(6231):222-225.

Omand, M. M. and Mahadevan, A. (2015). The shape of the oceanic nitracline. Biogeosciences, 12(11):3273.

Orsi, W. D., Smith, J. M., Liu, S., Liu, Z., Sakamoto, C. M., Wilken, S., Poirier, C., Richards, T. A., Keeling, P. J., Worden, A. Z., et al. (2016). Diverse, uncultivated bacteria and archaea underlying the cycling of dissolved protein in the ocean. The ISME Journal, 10(9):2158-2173.

Oschlies, A. (2002). Can eddies make ocean deserts bloom? Global Biogeochemical Cycles, 16(4):53-1.

Oubelkheir, K., Claustre, H., Sciandra, A., and Babin, M. (2005). Bio-optical and biogeochemical properties of different trophic regimes in oceanic waters. Liminology and Oceanography, 50(6):1795-1809.

Owens, S., Buesseler, K., Lamborg, C., Valdes, J., Lomas, M., Johnson, R., Steinberg, D., and Siegel, D. (2013). A new time series of particle export from neutrally buoyant sediments traps at the Bermuda Atlantic Time-series Study site. Deep Sea Research Part I: Oceanographic Research Papers, 72:34-47.

Palevsky, H. I. and Doney, S. C. (2018). How Choice of Depth Horizon Influences the Estimated Spatial Patterns and Global Magnitude of Ocean Carbon Export Flux. Geophysical Research Letters, 45(9):4171-4179.

Palevsky, H. I. and Nicholson, D. P. (2018). The North Atlantic biological pump: insights from the Ocean Observatories Initiative Irminger Sea array. Oceanography, 31(1):42-49.

Pallàs-Sanz, E., Johnston, T., and Rudnick, D. (2010). Frontal dynamics in a California Current System shallow front: 1. Frontal processes and tracer structure. Journal of Geophysical Research: Oceans, 115(C12).

Palter, J. B., Lozier, M. S., and Barber, R. T. (2005). The effect of advection on the nutrient reservoir in the North Atlantic subtropical gyre. Nature, 437(7059):687-692.

Pascual, A., Ruiz, S., Olita, A., Troupin, C., Claret, M., Casas, B., Mourre, B., Poulain, P.M., Tovar-Sanchez, A., Capet, A., Mason, E., Allen, J. T., Mahadevan, A., and Tintoré, J. (2017). A multiplatform experiment to unravel meso-and submesoscale processes in an intense front (AlborEx). Frontiers in Marine Science, 4:39.

Pasquero, C. (2005). Differential eddy diffusion of biogeochemical tracers. Geophysical Research Letters, 32(17).

Pasquero, C., Bracco, A., and Provenzale, A. (2005). Impact of the spatiotemporal variability of the nutrient flux on primary productivity in the ocean. Journal of Geophysical Research: Oceans, $110(\mathrm{C} 7)$. 
Perruche, C., Rivière, P., Lapeyre, G., Carton, X., and Pondaven, P. (2011). Effects of surface quasi-geostrophic turbulence on phytoplankton competition and coexistence. Journal of marine research, 69(1):105-135.

Plumb, R. (1979). Eddy fluxes of conserved quantities by small-amplitude waves. Journal of the Atmospheric Sciences, 36(9):1699-1704.

Plumb, R. and Mahlman, J. (1987). The zonally averaged transport characteristics of the GFDL general circulation/transport model. Journal of the Atmospheric Sciences, $44(2): 298-327$.

Poff, K. E., Leu, A. O., Eppley, J. M., Karl, D. M., and DeLong, E. F. (2021). Microbial dynamics of elevated carbon flux in the open ocean's abyss. Proceedings of the National Academy of Sciences, 118(4):e2018269118.

Poje, A. C., Özgökmen, T. M., Lipphardt, B. L., Haus, B. K., Ryan, E. H., Haza, A. C., Jacobs, G. A., Reniers, A., Olascoaga, M. J., Novelli, G., Griffa, A., Beron-Vera, F. J., Chen, S. S., Coelho, E., Hogan, P. J., Kirwan Jr., A. D., Huntley, H. S., and Mariano, A. J. (2014). Submesoscale dispersion in the vicinity of the Deepwater Horizon spill. Proceedings of the National Academy of Sciences, 111(35):12693-12698.

Pollard, R. T. and Regier, L. A. (1992). Vorticity and vertical circulation at an ocean front. Journal of Physical Oceanography, 22(6):609-625.

Polovina, J. J., Howell, E. A., and Abecassis, M. (2008). Ocean's least productive waters are expanding. Geophysical Research Letters, 35(3).

Pérez, V., Fernández, E., Marañón, E., Morán, X. A. G., and Zubkov, M. V. (2006). Vertical distribution of phytoplankton biomass, production and growth in the Atlantic subtropical gyres. Deep Sea Research Part I: Oceanographic Research Papers, 53(10):1616-1634.

Qiu, B., Chen, S., Klein, P., Sasaki, H., and Sasai, Y. (2014). Seasonal mesoscale and submesoscale eddy variability along the North Pacific subtropical countercurrent. Journal of Physical Oceanography, 44(12):3079-3098.

Quast, C., Pruesse, E., Yilmaz, P., Gerken, J., Schweer, T., Yarza, P., Peplies, J., and Glöckner, F. O. (2012). The SILVA ribosomal RNA gene database project: improved data processing and web-based tools. Nucleic Acids Research, 41(D1):D590-D596.

Ramachandran, S., Tandon, A., and Mahadevan, A. (2014). Enhancement in vertical fluxes at a front by mesoscale-submesoscale coupling. Journal of Geophysical Research: Oceans, 119(12):8495-8511.

Rasse, R., Dall'Olmo, G., Graff, J., Westberry, T. K., van Dongen-Vogels, V., and Behrenfeld, M. J. (2017). Evaluating optical proxies of particulate organic carbon across the surface Atlantic Ocean. Frontiers in Marine Science, 4:367.

Redi, M. H. (1982). Oceanic isopycnal mixing by coordinate rotation. Journal of Physical Oceanography, 12(10):1154-1158.

Reji, L., Tolar, B. B., Chavez, F. P., and Francis, C. A. (2020). Depth-differentiation and seasonality of planktonic microbial assemblages in the Monterey Bay upwelling system. Frontiers in Microbiology, 11:1075. 
Renault, L., Oguz, T., Pascual, A., Vizoso, G., and Tintoré, J. (2012). Surface circulation in the Alborán sea (western Mediterranean) inferred from remotely sensed data. Journal of Geophysical Research: Oceans, 117(C8).

Resplandy, L., Lévy, M., and McGillicuddy Jr, D. J. (2019). Effects of eddy-driven subduction on ocean biological carbon pump. Global Biogeochemical Cycles, 33(8):1071-1084.

Richardson, T. L. and Jackson, G. A. (2007). Small Phytoplankton and Carbon Export from the Surface Ocean. Science, 315(5813):838-840.

Rocha, C. B. (2015). pyspec.

Rodriguez, J., Tintoré, J., Allen, J. T., Blanco, J. M., Gomis, D., Reul, A., Ruiz, J., Rodríguez, V., Echevarría, F., and Jiménez-Gómez, F. (2001). Mesoscale vertical motion and the size structure of phytoplankton in the ocean. Nature, 410(6826):360-363.

Roshan, S. and DeVries, T. (2017). Efficient dissolved organic carbon production and export in the oligotrophic ocean. Nature Communications, 8(1):2036.

Rosso, I., Hogg, A. M., Strutton, P. G., Kiss, A. E., Matear, R., Klocker, A., and van Sebille, E. (2014). Vertical transport in the ocean due to sub-mesoscale structures: Impacts in the Kerguelen region. Ocean Modelling, 80:10-23.

Rotunno, R., Skamarock, W. C., and Snyder, C. (1994). An analysis of frontogenesis in numerical simulations of baroclinic waves. Journal of the Atmospheric Sciences, 51(23):33733398 .

Ruddick, B. and Richards, K. (2003). Oceanic thermohaline intrusions: observations. Progress in Oceanography, 56(3-4):499-527.

Ruiz, S., Claret, M., Pascual, A., Olita, A., Troupin, C., Capet, A., Tovar-Sánchez, A., Allen, J., Poulain, P.-M., Tintoré, J., and Mahadevan, A. (2019). Effects of oceanic mesoscale and submesoscale frontal processes on the vertical transport of phytoplankton. Journal of Geophysical Research: Oceans, 124(8):5999-6014.

Ruiz, S., Pascual, A., Garau, B., Pujol, I., and Tintoré, J. (2009). Vertical motion in the upper ocean from glider and altimetry data. Geophysical Research Letters, 36(14).

Sakamoto, C. M., Karl, D. M., Jannasch, H. W., Bidigare, R. R., Letelier, R. M., Walz, P. M., Ryan, J. P., Polito, P. S., and Johnson, K. S. (2004). Influence of Rossby waves on nutrient dynamics and the plankton community structure in the North Pacific subtropical gyre. Journal of Geophysical Research: Oceans, 109(C5).

Salmon, R. (1994). Generalized two-layer models of ocean circulation. Journal of Marine Research, 52(5):865-908.

Samelson, R. (1992). Fluid exchange across a meandering jet. Journal of Physical Oceanography, 22(4):431-444.

Sawyer, J. S. (1956). The vertical circulation at meteorological fronts and its relation to frontogenesis. Proceedings of the Royal Society of London. Series A. Mathematical and Physical Sciences, 234(1198):346-362. 
Shcherbina, A. Y. and D'Asaro, E. A. (2020). Direct observations of submesoscale modulation of ocean surface boundary layer turbulence. In Ocean Sciences Meeting 2020. AGU.

Shcherbina, A. Y., D'Asaro, E. A., Lee, C. M., Klymak, J. M., Molemaker, M. J., and McWilliams, J. C. (2013). Statistics of vertical vorticity, divergence, and strain in a developed submesoscale turbulence field. Geophysical Research Letters, 40(17):4706-4711.

Shcherbina, A. Y., Gregg, M. C., Alford, M. H., and Harcourt, R. R. (2009). Characterizing thermohaline intrusions in the North Pacific subtropical frontal zone. Journal of Physical Oceanography, 39(11):2735-2756.

Shcherbina, A. Y., Sundermeyer, M. A., Kunze, E., D’Asaro, E., Badin, G., Birch, D., Brunner-Suzuki, A.-M. E., Callies, J., Kuebel Cervantes, B. T., Claret, M., Concannon, B., Early, J., Ferrari, R., Goodman, L., Harcourt, R., Klymak, J. M., Lee, C. M., Lelong, M.-P., Levine, M. D., Lien, R.-C., Mahadevan, A., McWilliams, J. C., Molemaker, M. J., Mukherjee, S., Nash, J. D., Özgökmen, T., Pierce, S. D., Ramachandran, S., Samelson, R. M., Sanford, T. B., Shearman, R. K., Skyllingstad, E. D., Smith, K. S., Tandom, A., Taylor, J. R., Terray, E. A., Thomas, L. N., and Ledwell, J. R. (2015). The LatMix summer campaign: submesoscale stirring in the upper ocean. Bulletin of the American Meteorological Society, 96(8):1257-1279.

Siegel, D. A., Buesseler, K. O., Behrenfeld, M. J., Benitez-Nelson, C. R., Boss, E., Brzezinski, M. A., Burd, A., Carlson, C. A., D'Asaro, E. A., Doney, S. C., et al. (2016). Prediction of the export and fate of global ocean net primary production: The EXPORTS science plan. Frontiers in Marine Science, 3:22.

Siegel, D. A., Buesseler, K. O., Doney, S. C., Sailley, S. F., Behrenfeld, M. J., and Boyd, P. W. (2014). Global assessment of ocean carbon export by combining satellite observations and food-web models. Global Biogeochemical Cycles, 28(3):181-196.

Siegelman, L., Klein, P., Rivière, P., Thompson, A. F., Torres, H. S., Flexas, M., and Menemenlis, D. (2020). Enhanced upward heat transport at deep submesoscale ocean fronts. Nature Geoscience, 13(1):50-55.

Skyllingstad, E. D., Duncombe, J., and Samelson, R. M. (2017). Baroclinic frontal instabilities and turbulent mixing in the surface boundary layer. Part II: Forced simulations. Journal of Physical Oceanography, 47(10):2429-2454.

Smith, K. M., Hamlington, P. E., and Fox-Kemper, B. (2016). Effects of submesoscale turbulence on ocean tracers. Journal of Geophysical Research: Oceans, 121(1):908-933.

Sohrin, R., Isaji, M., Obara, Y., Agostini, S., Suzuki, Y., Hiroe, Y., Ichikawa, T., and Hidaka, K. (2011). Distribution of synechococcus in the dark ocean. Aquatic Microbial Ecology, 64(1):1-14.

Spall, M. A. (1995). Frontogenesis, subduction, and cross-front exchange at upper ocean fronts. Journal of Geophysical Research: Oceans, 100(C2):2543-2557.

Spiro Jaeger, G., MacKinnon, J., Lucas, A., Shroyer, E., Nash, J., Tandon, A., Farrar, J., and Mahadevan, A. (2020). How spice is stirred in the Bay of Bengal. Journal of Physical Oceanography, 50(9):2669-2688. 
Sprio Jaeger, G., Lucas, A. J., and Mahadevan, A. (2020). Formation of interleaving layers in the bay of bengal. Deep Sea Research Part II: Topical Studies in Oceanography, 172:104717.

Stamper, M. A., Taylor, J. R., and Fox-Kemper, B. (2018). The growth and saturation of submesoscale instabilities in the presence of a barotropic jet. Journal of Physical Oceanography, 48(11):2779-2797.

Stanley, R. H., McGillicuddy Jr, D. J., Sandwith, Z. O., and Pleskow, H. M. (2017). Submesoscale hotspots of productivity and respiration: Insights from high-resolution oxygen and fluorescence sections. Deep Sea Research Part I: Oceanographic Research Papers, 130:1-11.

Stephens, B. M., Opalk, K. L., Petras, D., Liu, S., Comstock, J., Aluwihare, L. I., Hansell, D. A., and Carlson, C. A. (2020). Organic matter composition at Ocean Station Papa affects its bioavailability, bacterioplankton growth efficiency and the responding taxa. Frontiers in Marine Science, 7:1077.

Stommel, H. (1963). Varieties of oceanographic experience. Science, 139(3555):572-576.

Stommel, H. (1979). Determination of water mass properties of water pumped down from the Ekman layer to the geostrophic flow below. Proceedings of the National Academy of Sciences, 76(7):3051-3055.

Stommel, H. and Arons, A. (1959). On the abyssal circulation of the world ocean-I. stationary planetary flow patterns on a sphere. Deep Sea Research (1953), 6:140-154.

Stramski, D., Shalapyonok, A., and Reynolds, R. A. (1995). Optical characterization of the oceanic unicellular cyanobacterium Synechococcus grown under a day-night cycle in natural irradiance. Journal of Geophysical Research: Oceans, 100(C7):13295-13307.

Stukel, M. R., Aluwihare, L. I., Barbeau, K. A., Chekalyuk, A. M., Goericke, R., Miller, A. J., Ohman, M. D., Ruacho, A., Song, H., Stephens, B. M., and Landry, M. R. (2017). Mesoscale ocean fronts enhance carbon export due to gravitational sinking and subduction. Proceedings of the National Academy of Sciences, 114(6):1252-1257.

Su, Z., Wang, J., Klein, P., Thompson, A. F., and Menemenlis, D. (2018). Ocean submesoscales as a key component of the global heat budget. Nature Communications, 9(1):775.

Sudek, S., Everroad, R. C., Gehman, A.-L. M., Smith, J. M., Poirier, C. L., Chavez, F. P., and Worden, A. Z. (2015). Cyanobacterial distributions along a physico-chemical gradient in the Northeastern Pacific Ocean. Environmental Microbiology, 17(10):3692-3707.

Sverdrup, H. (1953). On conditions for the vernal blooming of phytoplankton. J. Cons. Int. Explor. Mer, 18(3):287-295.

Tandon, A. and Garrett, C. (1994). Mixed layer restratification due to a horizontal density gradient. Journal of Physical Oceanography, 24(6):1419-1424.

Tandon, A. and Garrett, C. (1995). Geostrophic adjustment and restratification of a mixed layer with horizontal gradients above a stratified layer. Journal of Physical Oceanography, 25(10):2229-2241. 
Taylor, G. I. (1922). Diffusion by continuous movements. Proceedings of the London Mathematical Society, 2(1):196-212.

Taylor, J. R., Bachman, S., Stamper, M., Hosegood, P., Adams, K., Sallee, J.-B., and Torres, R. (2018). Submesoscale Rossby waves on the Antarctic Circumpolar Current. Science Advances, 4(3):eaao2824.

Taylor, J. R. and Ferrari, R. (2011). Shutdown of turbulent convection as a new criterion for the onset of spring phytoplankton blooms. Limnology and Oceanography, 56(6):2293-2307.

Taylor, J. R., Smith, K. M., and Vreugdenhil, C. A. (2020). The influence of submesoscales and vertical mixing on the export of sinking tracers in large-eddy simulations. Journal of Physical Oceanography, 50(5):1319-1339.

Thomas, L. N. (2008). Formation of intrathermocline eddies at ocean fronts by wind-driven destruction of potential vorticity. Dynamics of atmospheres and oceans, 45(3-4):252-273.

Thomas, L. N. and Joyce, T. M. (2010). Subduction on the northern and southern flanks of the Gulf Stream. Journal of Physical Oceanography, 40(2):429-438.

Thomas, L. N., Tandon, A., and Mahadevan, A. (2008). Submesoscale processes and dynamics. Ocean modeling in an Eddying Regime, 177:17-38.

Thomas, L. N., Taylor, J. R., Ferrari, R., and Joyce, T. M. (2013). Symmetric instability in the Gulf Stream. Deep Sea Research Part II: Topical Studies in Oceanography, 91:96-110.

Thompson, A. W., Kouba, K., and Ahlgren, N. A. (2021). Niche partitioning of low-light adapted Prochlorococcus subecotypes across oceanographic gradients of the North Pacific Subtropical Front. Limnology and Oceanography, 66(4):1548-1562.

Thomsen, S., Kanzow, T., Colas, F., Echevin, V., Krahmann, G., and Engel, A. (2016). Do submesoscale frontal processes ventilate the oxygen minimum zone off Peru? Geophysical Research Letters, 43(15):8133-8142.

Thurnherr, A. M., Kunze, E., Toole, J. M., St. Laurent, L., Richards, K. J., and Ruiz-Angulo, A. (2015). Vertical kinetic energy and turbulent dissipation in the ocean. Geophysical Research Letters, 42(18):7639-7647.

Tintore, J., La Violette, P., Blade, I., and Cruzado, A. (1988). A study of an intense density front in the eastern Alboran Sea: the Almeria-Oran front. Journal of Physical Oceanography, 18(10):1384-1397.

Torres, H. S., Klein, P., Menemenlis, D., Qiu, B., Su, Z., Wang, J., Chen, S., and Fu, L.-L. (2018). Partitioning ocean motions into balanced motions and internal gravity waves: A modeling study in anticipation of future space missions. Journal of Geophysical Research: Oceans, 123(11):8084-8105.

Treusch, A. H., Vergin, K. L., Finlay, L. A., Donatz, M. G., Burton, R. M., Carlson, C. A., and Giovannoni, S. J. (2009). Seasonality and vertical structure of microbial communities in an ocean gyre. The ISME Journal, 3(10):1148-1163. 
Tzortzis, R., Doglioli, A. M., Barrillon, S., Petrenko, A. A., d'Ovidio, F., Izard, L., Thyssen, M., Pascual, A., Barceló-Llull, B., Cyr, F., et al. (2021). Impact of moderate energetic finescale dynamics on the phytoplankton community structure in the western Mediterranean Sea. Biogeosciences Discussions, pages 1-38.

Uchida, T., Balwada, D., Abernathey, R. P., McKinley, G. A., Smith, S. K., and Lévy, M. (2020). Vertical eddy iron fluxes support primary production in the open Southern Ocean. Nature Communications, 11(1):1-8.

Vallis, G. (2006). Atmospheric and ocean fluid dynamics. Cambridge University Press, $6: 74-75$

Vergin, K., Done, B., Carlson, C., and Giovannoni, S. (2013a). Spatiotemporal distributions of rare bacterioplankton populations indicate adaptive strategies in the oligotrophic ocean. Aquatic Microbial Ecology, 71(1):1-13.

Vergin, K. L., Beszteri, B., Monier, A., Thrash, J. C., Temperton, B., Treusch, A. H., Kilpert, F., Worden, A. Z., and Giovannoni, S. J. (2013b). High-resolution SAR11 ecotype dynamics at the bermuda atlantic time-series study site by phylogenetic placement of pyrosequences. The ISME Journal, 7(7):1322-1332.

Verma, V., Pham, H. T., and Sarkar, S. (2019). The submesoscale, the finescale and their interaction at a mixed layer front. Ocean Modelling, 140:101400.

Vries, P. and Döös, K. (2001). Calculating Lagrangian trajectories using time-dependent velocity fields. Journal of Atmospheric and Oceanic Technology, 18(6):1092-1101.

Wang, D.-P. (1993). Model of frontogenesis: Subduction and upwelling. Journal of Marine Research, 51(3):497-513.

Washburn, L., Kadko, D. C., Jones, B. H., Hayward, T., Kosro, P. M., Stanton, T. P., Ramp, S., and Cowles, T. (1991). Water mass subduction and the transport of phytoplankton in a coastal upwelling system. Journal of Geophysical Research: Oceans, 96(C8):14927-14945.

Wenegrat, J. O., Thomas, L. N., Gula, J., and McWilliams, J. C. (2018). Effects of the submesoscale on the potential vorticity budget of ocean mode waters. Journal of Physical Oceanography, 48(9):2141-2165.

Wenegrat, J. O., Thomas, L. N., Sundermeyer, M. A., Taylor, J. R., D’Asaro, E. A., Klymak, J. M., Shearman, R. K., and Lee, C. M. (2020). Enhanced mixing across the gyre boundary at the Gulf Stream front. Proceedings of the National Academy of Sciences, 117(30):1760717614 .

White, A. E., Spitz, Y. H., and Letelier, R. M. (2007). What factors are driving summer phytoplankton blooms in the North Pacific subtropical gyre? Journal of Geophysical Research: Oceans, 112(C12).

Whitt, D., Lévy, M., and Taylor, J. R. (2017). Low-frequency and high-frequency oscillatory winds synergistically enhance nutrient entrainment and phytoplankton at fronts. Journal of Geophysical Research: Oceans, 122(2):1016-1041.

Woodwell, G. M. and Whittaker, R. H. (1968). Primary production in terrestrial ecosystems. American Zoologist, 8(1):19-30. 
Worden, A. Z., Nolan, J. K., and Palenik, B. (2004). Assessing the dynamics and ecology of marine picophytoplankton: the importance of the eukaryotic component. Liminology and Oceanography, 49(1):168-179.

Wright, T. D., Vergin, K. L., Boyd, P. W., and Giovannoni, S. J. (1997). A novel deltasubdivision proteobacterial lineage from the lower ocean surface layer. Applied and Environmental Microbiology, 63(4):1441-1448.

Wyatt, T. (2014). Margalef's mandala and phytoplankton bloom strategies. Deep Sea Research Part II: Topical Studies in Oceanography, 101:32-49.

Yin, W. and Huang, D. (2016). Evolution of submesoscale coastal frontal waves in the East China Sea based on geostationary ocean color imager observational data. Geophysical Research Letters, 43(18):9801-9809.

Yu, X., Naveira Garabato, A. C., Martin, A. P., Buckingham, C. E., Brannigan, L., and Su, Z. (2019). An annual cycle of submesoscale vertical flow and restratification in the upper ocean. Journal of Physical Oceanography, 49(6):1439-1461.

Zhou, K., Dai, M., Kao, S.-J., Wang, L., Xiu, P., Chai, F., Tian, J., and Liu, Y. (2013). Apparent enhancement of 234 Th-based particle export associated with anticyclonic eddies. Earth and Planetary Science Letters, 381:198-209. 